\title{
AN APTAMER-BASED SENSING PLATFORM FOR LUTEINISING HORMONE PULSATILITY MEASUREMENT
}

\author{
By \\ SHAOLIN LIANG \\ MRes, Imperial College London
}

A thesis submitted in partial fulfilment of the requirement for the degree of DOCTOR OF PHILOSOPHY (JOINT DEGREE)

at the

School of Biomedical Sciences, The University of Hong Kong and

Department of Medicine, Imperial College London

September 2018 


\section{Declarations of Originality}

I hereby declare that the work presented in this thesis is my own work, except where due acknowledgement is made, and it has not been previously included in a thesis, dissertation or report submitted to this University or to any other institution for a degree diploma or other qualification.

\section{Copyright Declarations}

The copyright of this thesis rests with the author and is made available under a Creative Commons Attribution Non-Commercial No Derivatives licence. Researchers are free to copy, distribute or transmit the thesis on the condition that they attribute it, that they do not use it for commercial purposes and that they do not alter, transform or build upon it. For any reuse or redistribution, researchers must make clear to others the licence terms of this work. 
Abstract of thesis entitled

\title{
"An aptamer-based sensing platform for luteinising hormone pulsatility measurement"
}

\author{
Submitted by \\ Shaolin Liang \\ For the degree of Doctor of Philosophy (Joint Degree) \\ Between The University of Hong Kong and Imperial College London \\ in September 2018
}

\begin{abstract}
Normal fertility in human involves highly orchestrated communication across the hypothalamic-pituitary-gonadal (HPG) axis. The pulsatile release of Luteinising Hormone ( $\mathrm{LH})$ is a critical element for downstream regulation of sex steroid hormone synthesis and the production of mature eggs. Changes in LH pulsatile pattern have been linked to hypothalamic dysfunction, resulting in multiple reproductive and growth disorders including Polycystic Ovary Syndrome (PCOS), Hypothalamic Amenorrhea (HA), and delayed/precocious puberty. Therefore, assessing the pulsatility of LH is important not only for academic investigation of infertility, but also for clinical decisions and monitoring of treatment. However, there is currently no clinically available tool for measuring human LH pulsatility. The immunoassay system is expensive and requires large volumes of patient blood, limiting its application for LH pulsatility monitoring.
\end{abstract}

In this thesis, I propose a novel method using aptamer-enabled sensing technology to develop a device platform to measure LH pulsatility. I first generated a novel aptamer binding molecule against LH by a nitrocellulose membrane-based in vitro selection then characterised its high affinity and specific binding properties by multiple biophysical/chemical methods. I then developed a sensitive electrochemical-based detection method using this aptamer. The principal mechanism is that structure switching upon binding is associated with the electron 
transfer rate changes of the MB redox label. I then customised this assay to numerous device platforms under our rapid prototyping strategy including 96 well automated platform, continuous sensing platform and chip-based multiple electrode platform. The best-performing device was found to be the AELECAP (Automated ELEctroChemical Aptamer Platform) - a 96-well plate based automatic micro-wire sensing platform capable of measuring a series of low volume luteinising hormone within a short time.

Clinical samples were evaluated using AELECAP. A series of clinical samples were measured including LH pulsatility profile of menopause female (high LH amplitude), normal female/male (normal LH amplitude) and female with hypothalamic amenorrhea (no LH pulsatility). Total patient numbers were 12 of each type, with 50 blood samples collected every 10 mins in 8 hours. Results showed that the system can distinguish LH pulsatile pattern among the cohorts and pulsatility profiles were consistent with the result measured by clinical assays.

AELECAP shows high potential as a novel approach for clinical aptamer-based sensing. AELECAP competes with current automated immunometric assays system with lower costs, lower reagent use, and a simpler setup. There is potential for this approach to be further developed as a tool for infertility research and to assist clinicians in personalised treatment with hormonal therapy.

Thesis Supervisors: Dr. Julian Tanner, Prof. Waljit Dhillo and Prof. Tony Cass 


\section{Dedication}

To my parents Weiguo Liang, Mingyin Cai, my wife Chen Liu, and my lovely daughter Anwen Liang

For their sacrifices, encouragement, and love. 


\section{Acknowledgements}

I've been fortunate to have numerous positive influences during my graduate studies. Without these incredible mentors, colleagues, and friends, I would never achieve what I have done within this five years not only for my research but also my personal life.

Firstly I would like to thank my HKU supervisor Dr Julian Tanner. During my candidature, he allowed me the freedom of pursuing my favourite academic research and entrepreneurial projects, as well as encouragements and supports I needed for my new family. Julian is also one of my most respective "senior alumni" from Imperial College London, the first meeting I had with him at the Starbuck outside Gloucester Road station next to the college was particularly memorable. We talked about everything related to aptamers, diagnostics, therapeutics, etc. Since then, we had many times discussion similar in a more relaxed atmosphere (not just sitting inside the office). It's all these great moments make me feel it's my fortune to work with him. Without the help of him, it won't be possible for me to setup this Joint $\mathrm{PhD}$ project.

I would like to thank my Principle Imperial College supervisor Professor Waljit Dhillo. He brought me into the wonderful Kisspeptin world. I won't forget the first time when I heard Waljit explain the naming story of KISS1 gene to me with a mug printed with Hershey's Kisses chocolate brand logo. (Kisspeptin was named because of the location of where it was discovered - Hershey - the home of Kisses chocolate). I have never met anyone so enthusiastic about the prospects of the field on which he was working - I can see the excitement from his eyes when he told me that the clinical trial of using Kisspeptin for infertility treatment was successful and showed me the photo of the firstborn Kisspeptin baby in the world. That is what a true clinical scientist about - using his knowledge to make patients a better life.

I would also like to thank my Secondary Imperial College supervisor and my MRes supervisor Professor Tony Cass. I've been working with Tony since my Master period for around six years. It is he taught me the word "aptamer" and guided me to enter this extraordinary field. He also offered me numerous supports for all my entrepreneurial activities at Imperial College. His insightful advice 
helped me a lot regarding to further explore the opportunity of bridging the gap between my academic and industrial experience. I am very grateful that he gave me the chance to link up this Joint $\mathrm{PhD}$ program within all these three different labs and supported my non-study leave during my daughter's birth.

I am lucky to have the opportunity of studying at a place as productive and resourceful as HKU and Imperial College and in a program as unique as the Joint $\mathrm{PhD}$. Studying with talented people from all the three labs was an extraordinary experience. Therefore, I want to have my sincere gratefulness to all the members in these three labs. Thank people from Julian's lab for all their supports: Dr Yee-Wai Cheung, Dr Lina Li, Dr Yuanyuan Yu, Dr Andrew Kinghorn, Dr Roderick Dirkzwager, Meili Khong, Lewis Fraser, Simon Shiu, Chandra Jinata, Soubhagya Bhuyan, Mengping Liu and Vanessa Choi. Especially for Andrew, Roderick, and Lewis - my coffee club mates!; Thank Julia Prague from Waljit's lab for all her support on the clinical sample collection works; Thank people from Tony's lab for all their long accompany and advice since my Master: Dr Thao Le, Dr Sanjiv Sharma, Dr Christopher Johnson, Dr Hanadi Hassan-Nixon, Dr Nikolaos Daskalakis, Dr Benjamin Miles, Dr Daniel Wilkinson, Dr Jasmine Sze, Ascanio Digiacomo, Yu Zhou, and Hannah Somani. Especially for Thao, she was my first teacher on aptamer selection and helped me a lot with my experiments. Also Ben and Dan, who are my best mates for drinking!

Most of all, I would like to thank my family for supporting me so far. My parents raised me to look beyond superficial achievements and used themselves as an example to teach me what a successful life should be. They are my superheroes! I am standing behind them and looking forward to becoming them one day with my own family..

That's why I have to save the last acknowledgement to my beautiful wife Chen Liu. She has sacrificed so much to support my study especially after we have our lovely daughter Anwen Liang. Without the immense support, I will never ever get to here. Here in China, we have an old cliché saying said by Confucius being "at thirty, I became independent and stood firm (三十而立)”. Soon will be my 30year-birthday, I am about to stand firm and I hope to thank all the people that shaped me what I am now and made me stand how I should be during these 30 years lifetime. 


\section{Research output during candidature}

\section{Peer-Reviewed Research Publications:}

Tang,M.S.L., Shiu,S.C.C., Godonoga,M., Cheung,Y.W., Liang,S., Dirkzwager, R.M., Kinghorn,A.B., Fraser,L.A., Heddle,J.G. \& Tanner,J.A. An aptamer-enabled DNA nanobox for protein sensing. Nanomedicine (2018)

Kinghorn,A.B., Fraser,L.A., Liang,S., Shiu,S.C.C and Tanner,J.A. Aptamer Bioinformatics. IJMS (2017)

Fraser,L.A., Kinghorn,A.B., Dirkzwager, R.M., Cheung,Y.W., Liang,S., Lim,B., Shiu,S.C.C., Tang,M.S.L., Andrew,D., Manitta,J., Richards,J.S \& Tanner,J.A. A Portable Microfluidic Aptamer-Tethered Enzyme Capture (APTEC) biosensor for malaria diagnosis. Biosensors and Bioelectronics (2017)

Shiu,S.C.C., Cheung,Y.W., Dirkzwager, R.M., Liang,S., Kinghorn,A.B., Fraser,L.A., Tang,M.S.L., and Tanner,J.A. Aptamer-Mediated Protein Molecular Recognition Driving a DNA Tweezer Nanomachine. Advanced Biosystems (2017)

(Co-first author) Dirkzwager,R.M., Liang,S., \& Tanner,J.A. Development of aptamer-based point-of-care diagnostic device for malaria using 3D printing rapid prototyping. ACS Sensors (2016)

Kinghorn,A.B., $\quad$ Dirkzwager,R.M., $\quad$ Liang,S., $\quad$ Cheung,Y.W., Fraser,L.A., Tang,M.S.L \& Tanner,J.A. Aptamer Affinity Maturation by Resampling and Microarray Selection. Analytical Chemistry (2016)

Fraser, L.A., Kinghorn,A.B., Tang, M.S.L., Cheung,Y.W., Lim,B., Liang.S., Dirkzwager, R.M. \& Tanner,J.A. Oligonucleotide functionalized microbeads: indispensable tools for high-throughtput aptamer selection. Molecules (2015) 


\section{Conference Presentation:}

Liang,S., Kinghorn,A.B., Prague,J.K., Dhillo,W.S., Cass, A.E.G., Li, R.H.W. \& Tanner,J.A. Automated electrochemical sensing system for monitoring of luteinising hormone pulsatility. Aptamers Oxford (2018)

Liang,S., Kinghorn,A.B., Dhillo,W.S., Cass, A.E.G. \& Tanner,J.A. Continuous flow electrochemical sensing system for monitoring luteinising hormone pulsatility. Oligos Oxford (2018)

Liang,S., Kinghorn,A.B., Dhillo,W.S., Cass, A.E.G. \& Tanner,J.A. Development of an aptamer-enable electrochemical sensing system for luteinising hormone pulsatility measurement. Aptamer in bordeaux (2016)

Liang,S., Dirkzwager,R.M. \& Tanner,J.A. Rapid Prototyping Aptamer-Enabled Malaria Diagnostics Using Three-Dimensional Printing. Aptamer Oxford (2016)

\section{Paper in preparation:}

Liang.S., Kinghorn,A.B., Prague,J.K., Dhillo,W.S., Cass, A.E.G., Li, R.H.W. \& Tanner,J.A. AELECAP - a Low-cost, automated aptamer-based sensing systems for human Luteinizing Hormone pulsatility monitoring 


\section{Contents}

CHAPTER 1 | Motivations for measuring Luteinising Hormone pulsatility 16

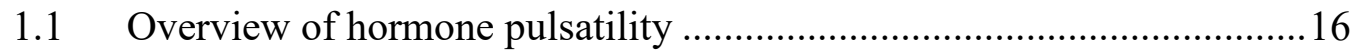

1.2 The significance of GnRH and LH pulsatility for reproduction .............18

1.2.1 Pulsatile hormone regulations in HPG axis ..................................19

1.2.2 Clinical implications of pulsatile LH signals................................25

1.3 Current methods and challenges for measuring LH pulsatility...............30

1.3.1 Blood sampling methods ...............................................................30

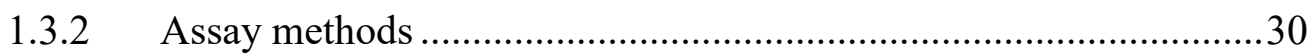

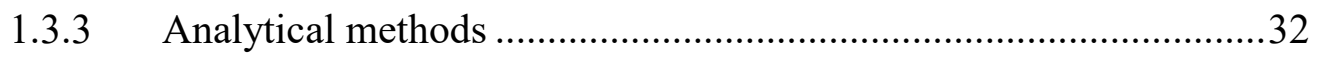

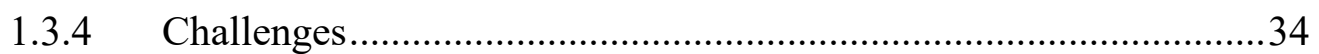

1.4 Aptamer-enable sensor for LH pulsatility measurement ........................35

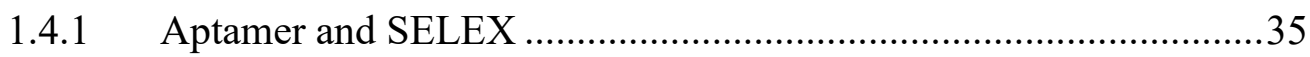

1.4.2 Electrochemical-based aptasensor ...................................................37

1.4.3 Advantage of using E-AB platform for LH pulsatility measurement 39

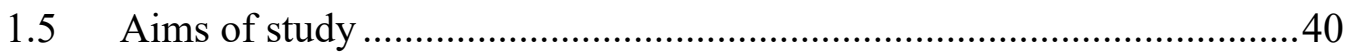

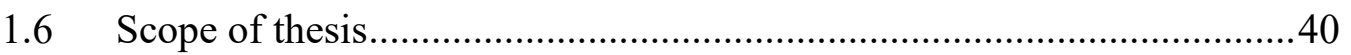

CHAPTER 2 | Generation of LH specific aptamer ............................................42

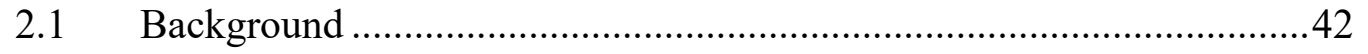

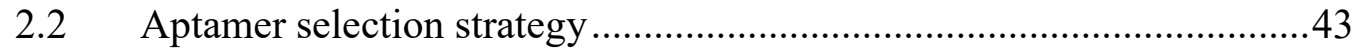

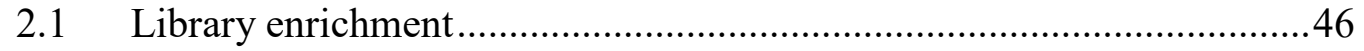

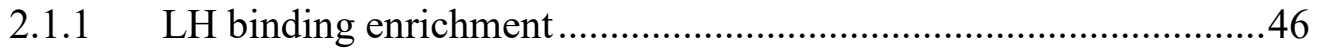

2.1.2 Further affinity and specificity characterisation of 20R library ......47

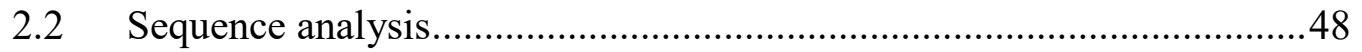

2.2.1 Cloning and Sanger sequencing .....................................................4

2.3 Affinity and specificity characterization for picked candidates..............49

2.3.1 Screening of best candidate by ELONA .........................................49

2.3.2 Truncation study of selected aptamer ...........................................50

2.3.3 Specificity study of selected aptamer ...........................................51

2.4 Binding kinetics and conformational changing dynamics studies ..........52 
2.4.1 Surface Plasmon Resonance (SPR) study for selected aptamer ......52

2.4.2 Circular Dichroism (CD) study for selected aptamer .....................53

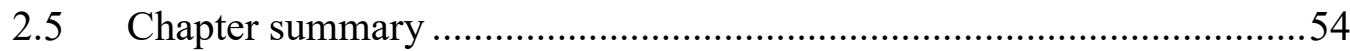

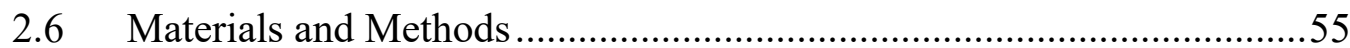

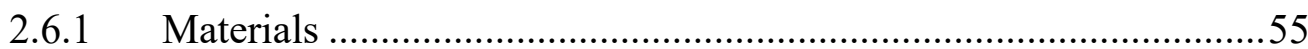

2.6.2 Nitrocellulose membrane filtration ..............................................56

2.6.3 PCR amplification and agarose gel electrophoresis ......................56

2.6.4 Generation of ssDNA by Biotin-streptavidin separation.................57

2.6.5 Enzyme Linked OligoNucleotide Assay (ELONA) .......................57

2.6.6 Electrophoretic Mobility Shift Assay (EMSA) ..............................58

2.6.7 Cloning and sequencing ............................................................58

2.6.8 Surface Plasmon Resonance (SPR) .............................................58

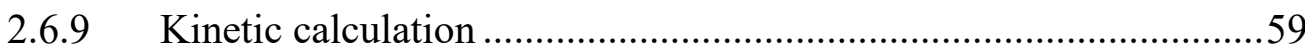

2.6.10 Circular Dichroism (CD) ........................................................59

CHAPTER 3 | Electrochemical aptamer-based LH assay development .......60

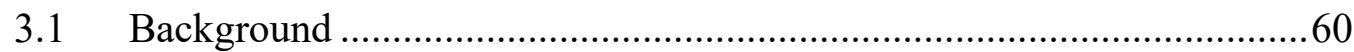

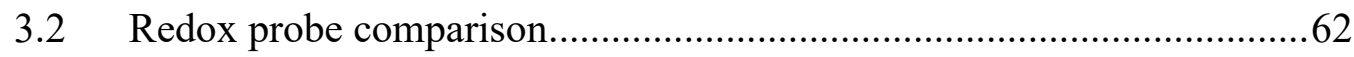

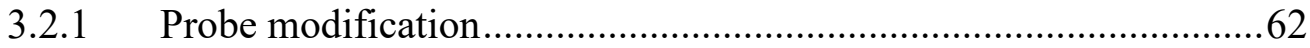

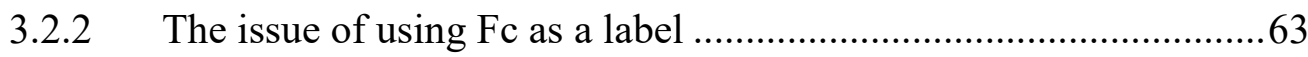

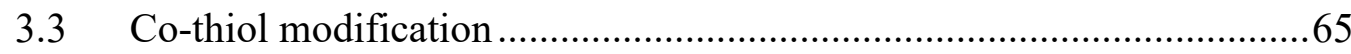

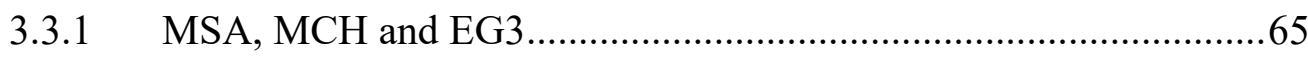

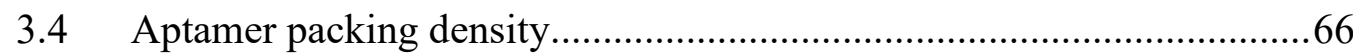

3.4.1 Roughness factor (RF) of different electrodes...............................66

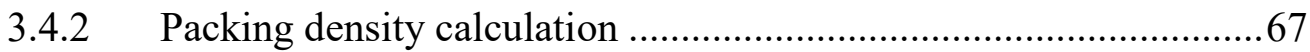

3.5 Electron transfer kinetic characterisation ...........................................68

3.5.1 Coefficient constant and charge transfer rate constant calculation..68

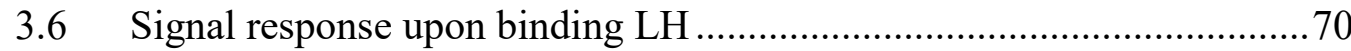

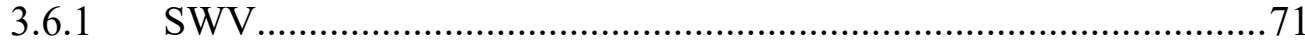

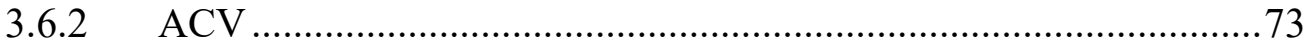

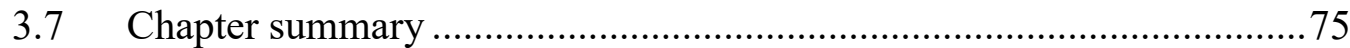

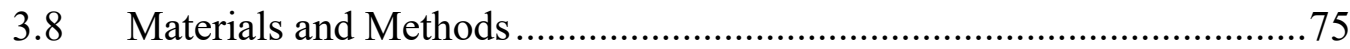

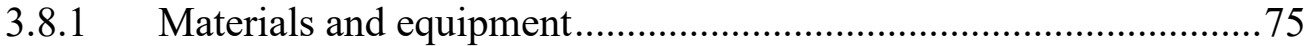


3.8.2 Chemical modification of B23 with redox group .........................76

3.8.3 Electrochemical experiment setup ................................................... 76

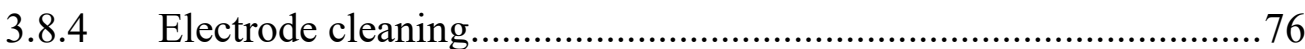

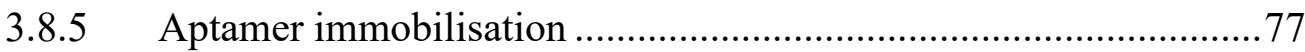

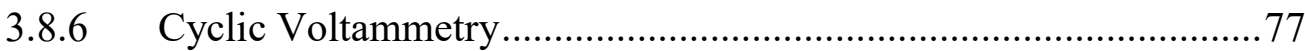

3.8.7 Square-wave Voltammetry ........................................................... 78

3.8.8 Alternative Cyclic Voltammetry..................................................... 78

CHAPTER 4 | Rapid prototyping devices for the electrochemical assay......79

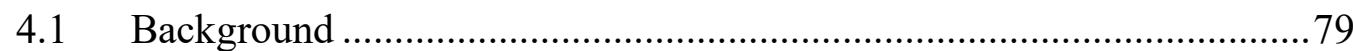

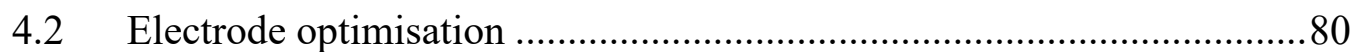

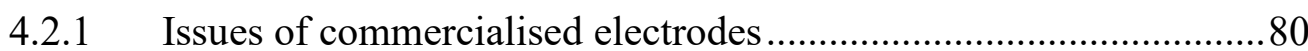

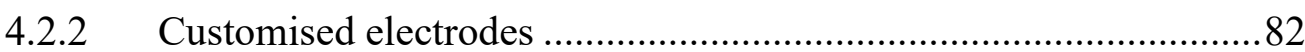

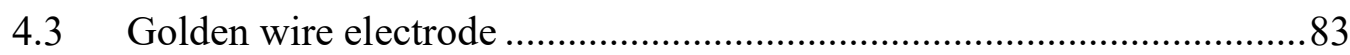

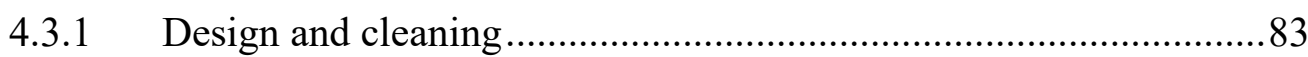

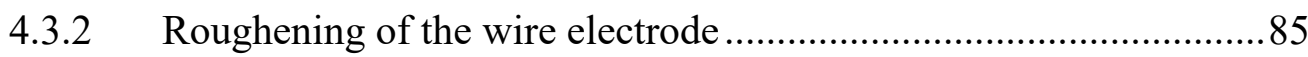

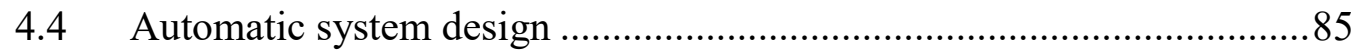

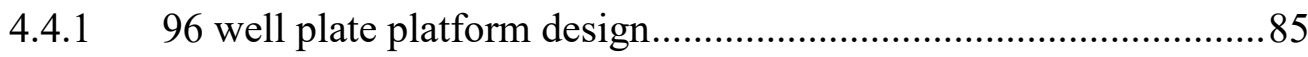

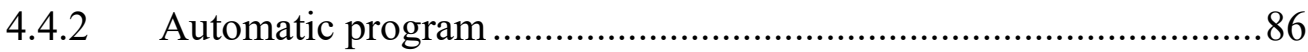

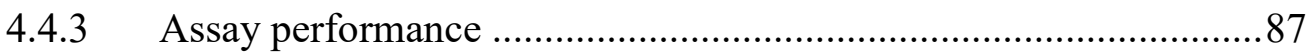

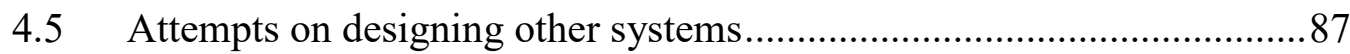

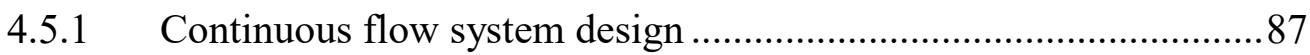

4.5.2 Multiple-electrode chip based design .......................................... 88

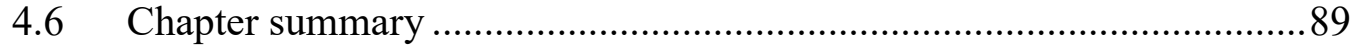

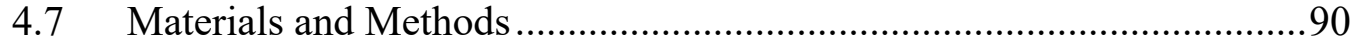

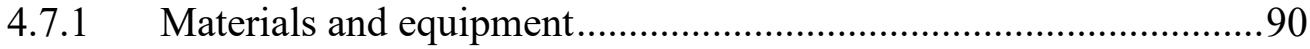

4.7.2 Sensor fabrication in commercialised electrodes............................91

4.7.3 Sensor fabrication in customised electrodes ..................................91

4.7.4 Fabrication of the gold wire electrode .........................................91

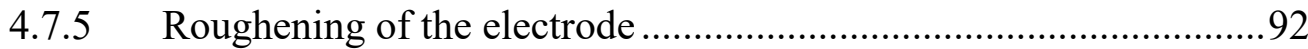

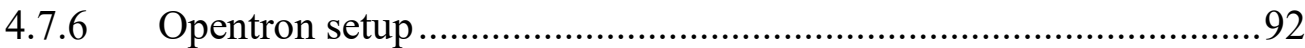

CHAPTER 5 | Clinical evaluation of the automatic platform in patient samples with different LH pulsatility profiles..................................................93 


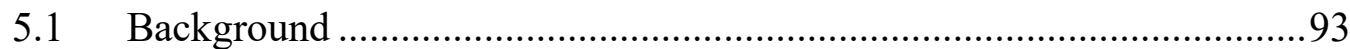

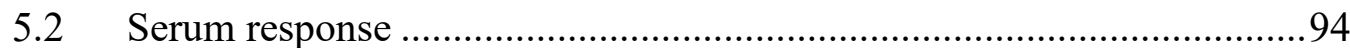

5.2.1 Conversion of the molar concentration to the international unit .....95

5.2.2 Electrochemical signal response in undiluted serum ......................95

5.2.3 Regeneration in a serum sample ...................................................96

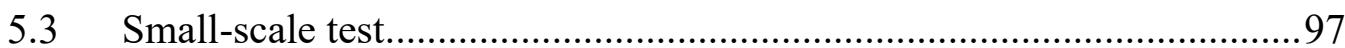

5.4 Menopause patient samples (high LH level) .......................................98

5.5 Health people samples (normal LH level) ............................................ 100

5.5.1 Serum samples collected from healthy female .............................100

5.5.2 Serum sample collected from healthy male ..................................101

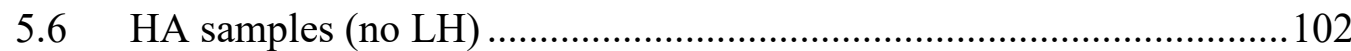

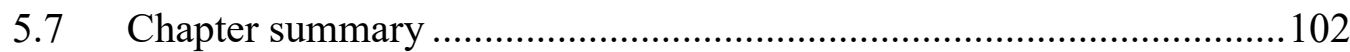

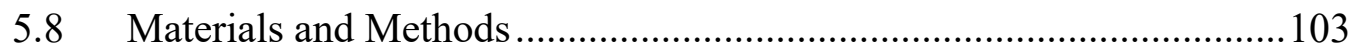

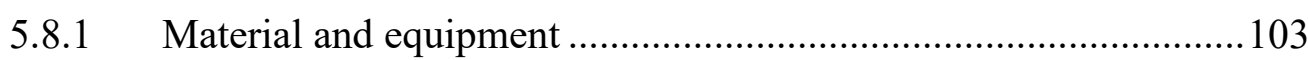

5.8.2 Automatic assay procedure ….................................................... 104

CHAPTER 6 | Perspectives and outlook........................................................105

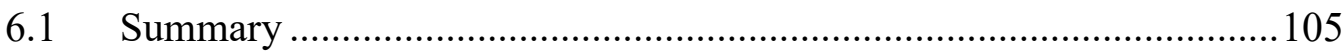

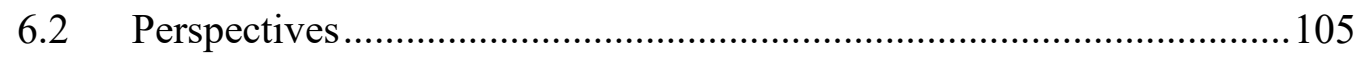

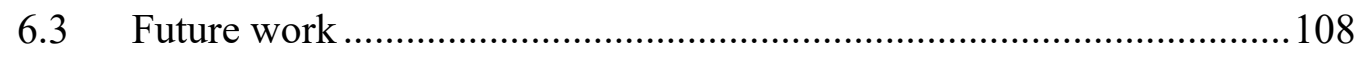

\section{APPENDIX}

Request Letters for copyright permission

Conference abstracts and research publications 


\section{List of Figures}

Figure 1-1: Overview of hormone regulations and pulsatile secretion patterns in major

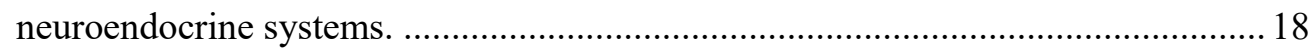

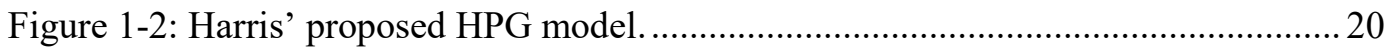

Figure 1-3: Correlation between GnRH and LH pulsatile secretion.................................. 21

Figure 1-4: Proposed model for regulation of signalling by the GnRH receptor in response to the tonic (left) or pulsatile (right) GnRH. .......................................... 22

Figure 1-5: Proposed model for pulse regulation of the LH $\beta$ and FSH $\beta$ promoters......... 23

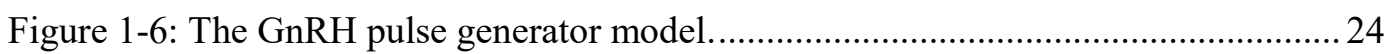

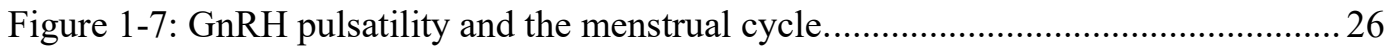

Figure 1-8: Indications for LH pulsatile secretion pattern. ............................................ 27

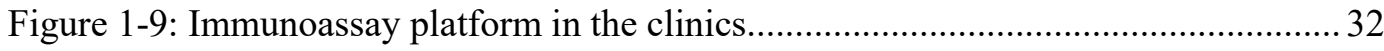

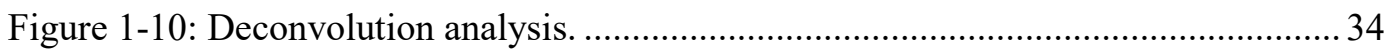

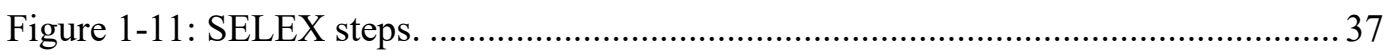

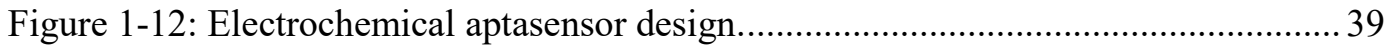

Figure 2-1: LH SELEX and the expected properties of the aptamer. ............................... 43

Figure 2-2: Nitrocellulose membrane-based SELEX ....................................................... 45

Figure 2-3: ELONA study for affinity enrichment. ........................................................ 46

Figure 2-4: EMSA study to further confirm the binding affinity and specificity. ............ 48

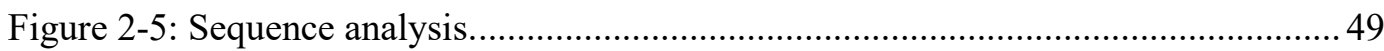

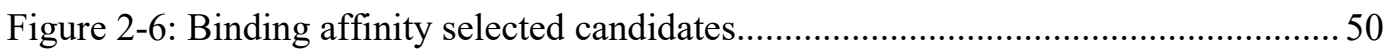

Figure 2-7: Binding affinity and specificity of selected candidates.................................51

Figure 2-8: SPR study to investigate the binding kinetics of B23.................................53

Figure 2-9: Conformational study on aptamer candidates ............................................5 54

Figure 3-1: Commercially available redox modifiers. (A) Anthraquinone, ....................... 62

Figure 3-2: Blank SWV signal of MB modified aptamer and Fc modified aptamer on gold disk electrode. 
Figure 3-3: Stability comparison between the SWV peak signal generated by MB and Fc

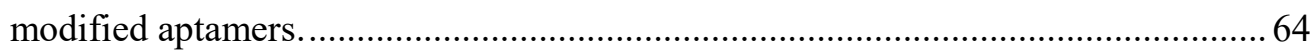

Figure 3-4: Unknown peaks for Fc modified aptamer on chip electrodes .......................65

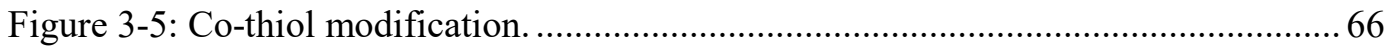

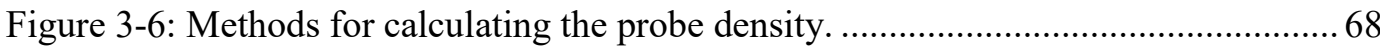

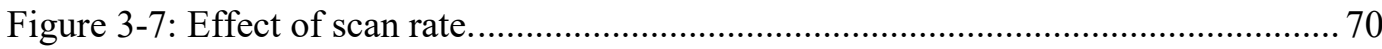

Figure 3-8: Signal response change under different SWV frequency. ............................ 72

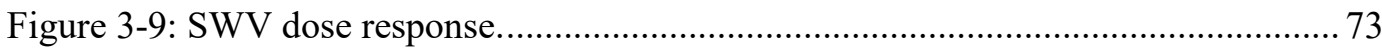

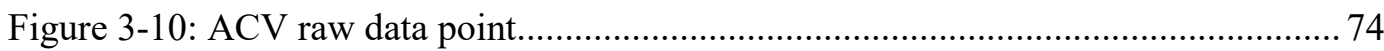

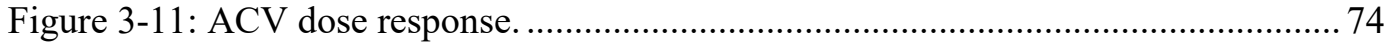

Figure 4-1: Investigation of Dropsens and Micrux electrode. ……..................................... 82

Figure 4-2: Investigation of physical deposited and chemical deposited electrode........... 83

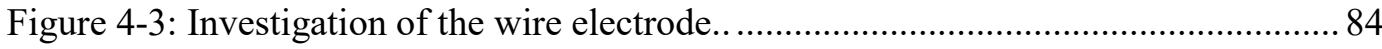

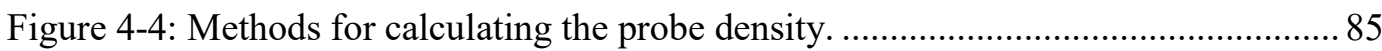

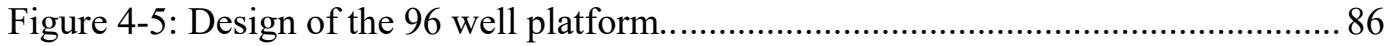

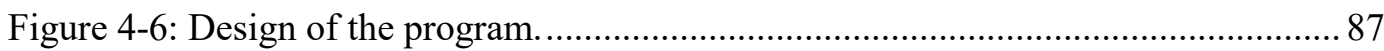

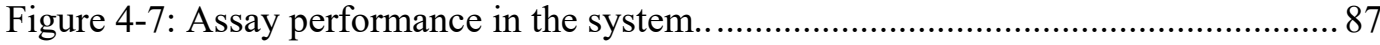

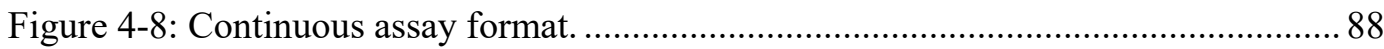

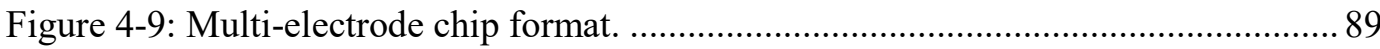

Figure 5-1: Methods for calculating the probe density.................................................... 94

Figure 5-2: Dose-response of the sensing system in undiluted serum.............................96

Figure 5-3: Regeneration under undiluted serum condition............................................. 97

Figure 5-4: Pilot studies on measuring LH pulsatility. ....................................................98

Figure 5-5: LH pulsatility measurement of menopause female samples. ......................... 99

Figure 5-6: LH pulsatility measurement of healthy female samples.............................. 100

Figure 5-7: LH pulsatility measurement of healthy male samples.................................. 101

Figure 5-8: Three HA patients' LH pulsatility profile...................................................... 102 


\section{CHAPTER 1 | Motivations for measuring Luteinising Hormone pulsatility}

In this introductory chapter, I discuss the motivations behind the research project. First, I discuss the implications of pulsatile hormone secretion on the reproductive axis and describe why I think Luteinising Hormone (LH) pulsatility is an optimal marker for neuroendocrinology research and anovulatory infertility diagnosis. I also discuss what are the existing methods and critical challenges for LH pulsatility measurement and bring out the aptamer-based sensing technology as a promising solution to address some of these challenges. Finally, I summarise the scope of the thesis and list the critical scientific questions I aim at tackling in the rest thesis chapters.

Keywords: HPG axis, Luteinising Hormone pulsatility, Electrochemical aptamerbased sensor

\subsection{Overview of hormone pulsatility}

The earliest usage of the word "pulsatility" was found in 1836 in Robert Bentley Todd's Cyclopaedia of Anatomy and Physiology. At the time, this word has not yet been linked to neuroendocrinology - it was referred to the rhythmic characteristics of a bone tumour observed during physician's examination [1]. In 1905, Ernest Starling invented the word "hormone" (meaning 'to arouse and excite' in Geek) in his lecture and defined it as the blood travelling chemical messengers that coordinate the activities throughout the entire body. Later, this concept of "hormone" has sparked a wide range of research areas and led to the establishment of endocrinology and neuroendocrinology [2].

However, three decades would elapse before the first experimental observation of the actual pulsatile hormone secretion in animal [3]. Since then, progressively more evidence has been found to support the importance of hormone pulsatility in regulating neuroendocrine systems. The word "Pulsatile" represents the reappearance of specific interposed events such as pulse interrupting a constant baseline process [4]. Therefore, the pulsatile secretion of hormone is the biochemical event which refers to this hormone is secreted in a burst-like or 
episodic rhythm manner rather than a consistent format to generate signals for the regulation of downstream factors.

After the development of this endocrinology and neuroendocrinology over a century, we now understand our neuroendocrine systems control many of the most fundamental physiological processes via "hormone communicating networks" involving hypothalamus, pituitary and specific end organ/gland. Figure 1-1 A illustrates three of the most well-known endocrine axes response in regulating reproduction, stress and body growth. The majority of hormones such as Gonadotropin-releasing hormone $(\mathrm{GnRH})$ [5], growth hormone $(\mathrm{GH})$ [6], adrenal glucocorticoids [7] and gonadal sex steroids [8] in these axes are secreted in a pulsatile fashion, and some of them involve complicated communicating mechanisms based on the recognition of different pulse amplitudes and frequencies [9]. The alterations of these pulsatile patterns may link with diseases. For example, hypogonadotropic hypogonadism happens when the GnRH pulses are abolished [10], and growth failure may be triggered when growth hormone releasing hormone receptor's function is abrogated [11]. Figure 1-1 B illustrates the profiles of pulsatile hormone secretion pattern of LH, testosterone (Te), Adrenocorticotropic hormone $(\mathrm{ACTH})$ and $\mathrm{GH}$ in different physiological and pathological conditions.

As hormones play an essential role in physiological processes that highly relevant to our daily health, a better understanding of their pulsatile secretion fashion become significant not only in the perspective of expanding scientific advance, but also improving healthcare management. In the later sections, I will mainly focus on elucidating the reproductive axis and discuss the importance and challenges of measuring luteinising hormone pulsatility, as well as what in this project I am going to add on addressing these challenges. 

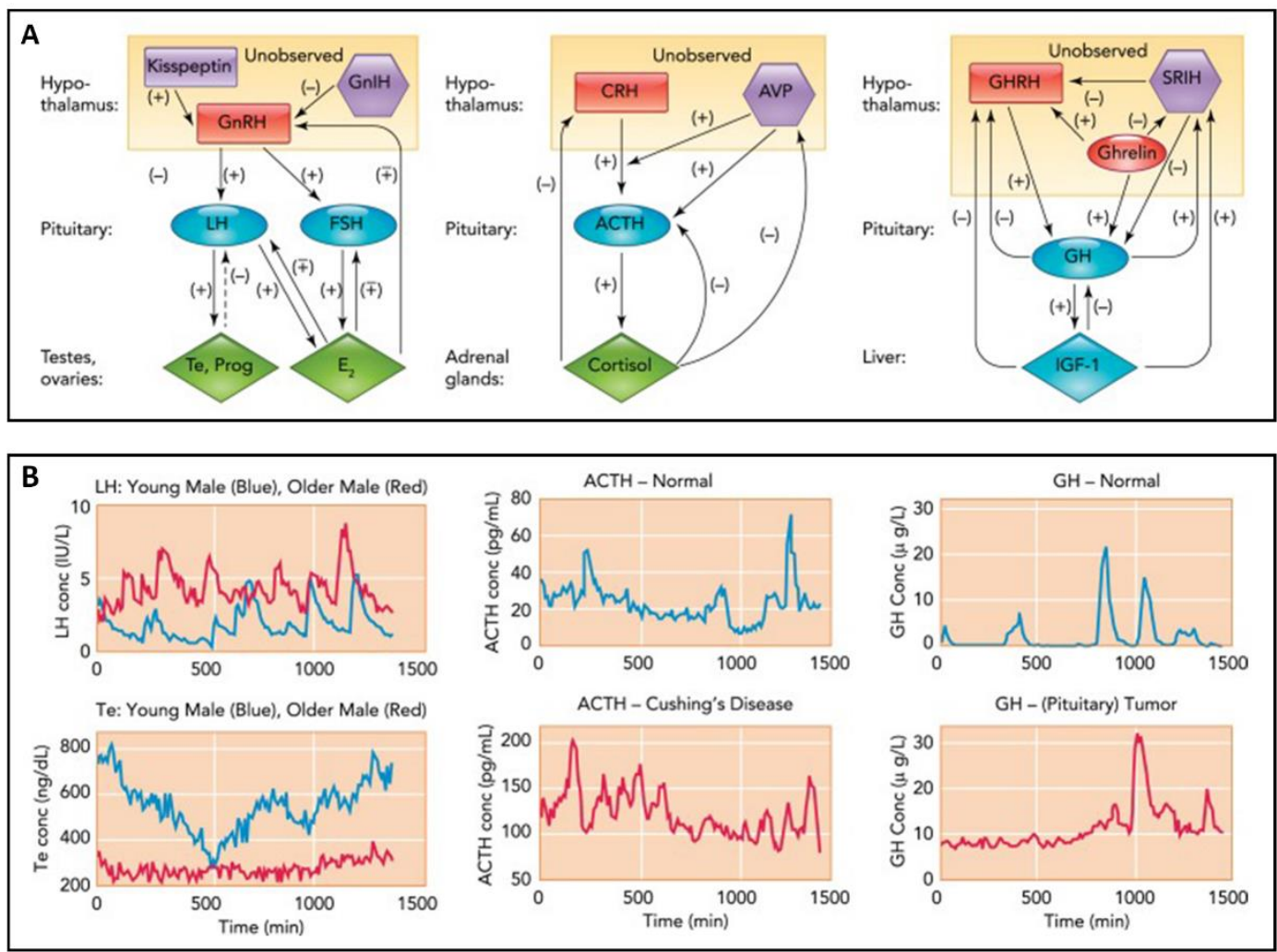

Figure 1-1: Overview of hormone regulations and pulsatile secretion patterns in major neuroendocrine systems. Figure adapted from ref. [12] (A) Illustration of the hormone regulation in axis response in regulating reproduction (left), stress control (middle), and body growth (right). The yellow boxes represent those hormones pulsatility cannot be directly measured in human clinical feasibly as they are in the hypothalamus. (B) Pulsatility profiles of key hormones in the above axes. The red and blue colour curves represent samples in different physiological or pathological conditions. A clear difference can be observed.

\subsection{The significance of GnRH and LH pulsatility for reproduction}

The pulsatile release of GnRH and its downstream hormone LH are two of the critical elements in the signalling communication of Hypothalamic-pituitarygonadal (HPG) axis for the reproduction of sex steroid hormones synthesis and the generation of mature eggs. In this section, I describe the hormone regulations within the HPG axis and the clinical implications of pulsatile GnRH and LH secretion. I aim at answering, from the basic, why I think measuring LH pulsatility is important. 


\subsubsection{Pulsatile hormone regulations in HPG axis}

\section{Early hypothesis}

The neuroendocrine system consists of numerous feedback loop axes mediated by hypothalamus and pituitary gland. HPG system is one of the most central axes relevant to reproductive regulation (Figure 1-1 A, top left). Central to the HPG axis are two pituitary hormones: LH and follicle-stimulating hormone (FSH). The upstream GnRH regulates both of these two hormones for the function of stimulating the downstream gonads to secrete sex steroids (testosterone in male, progesterone and estradiol in female) for reproductive development. The very first recognition of the character of LH and FSH to gonadal function dates back to 1929 when Fluhmann investigated the anterior pituitary hormone in women with ovarian deficiency [13]. It was a decade before Geoffrey Harris proposed the renowned hypothesis that hypothalamus involves the neural mechanisms controlling the pituitary-gonadal axis [14] (Figure 1-2).

In his theory, he suggested two general points: 1) the neuronal systems within the hypothalamus are capable of synthesising and secreting so-called "humoral substance" which to the pituitary gland via hypothalamic-hypophyseal portal circulation, thus regulate the secretion of pituitary hormone (gonadotrophic hormone in the original word); 2) There is a negative feedback mechanism exists in regulating the upstream "humoral substance" secretion from the gonadal steroid hormone.

In the history, it was the first-time people connect hypothalamus, pituitary and gonadal gland and recognise them as an entire system. However, at that time, what exactly this substance is and how does it regulate in this negative feedback loop remain rarely known. 


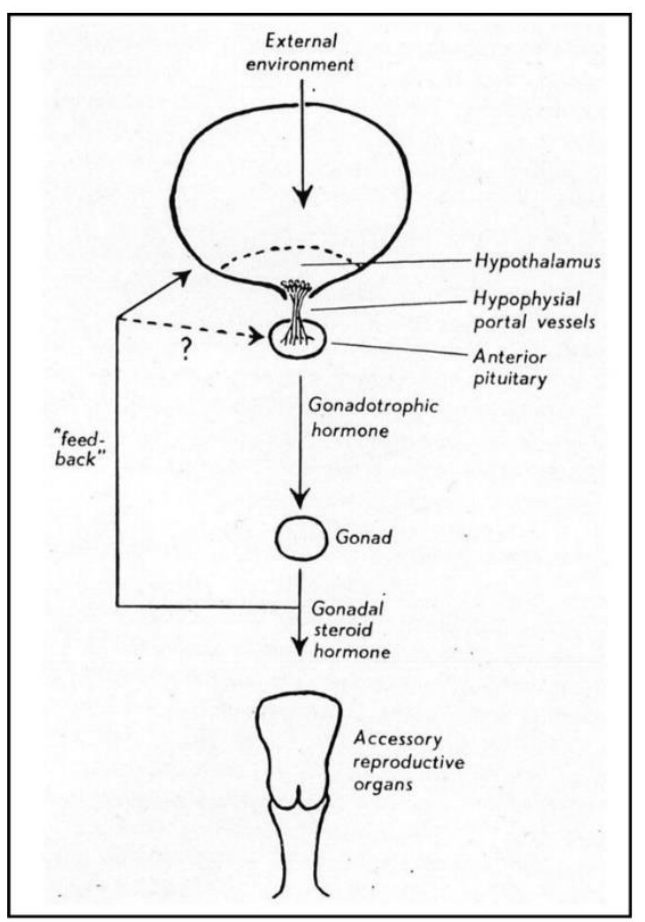

Figure 1-2: Harris' proposed HPG model. Figure adapted from ref. [14] In the monograph Geoffrey Harris published in 1955, he illustrated the linkage between the outside environment and the rest of reproductive organs. The question mark in the middle represents the limited knowledge at that time for people knowing the negative feedback control in the HPG axis.

\section{The correlation between pulsatile secretion of GnRH and LH}

A decade after Harris' publication, the "humoral substance", currently wellrecognised as $\mathrm{GnRH}$, was successfully isolated from ovine and bovine brain by the groups of Guilleman and Schally $[15,16]$. Soon after this, the pulsatile correlation between GnRH and LH was revealed by multiple studies. In two studies conducted by Knobil's group on rhesus monkey model, they found out intermittent stimulation of GnRH to the pituitary is critical to sustain LH and FSH level [17, 18] (Figure 13 A). This indicated GnRH may be secreted in a pulsatile format and this pulsatile release of GnRH stimulates the pulsatile release of LH. Clarke experimentally confirmed this direct correlation by measuring GnRH under the hypothalamichypophyseal portal circulation and also LH under systemic circulation in an animal model [19]. As can be seen in Figure 1-3 B, they found out each of the LH pulses is correlated with the GnRH pulse being recorded. 


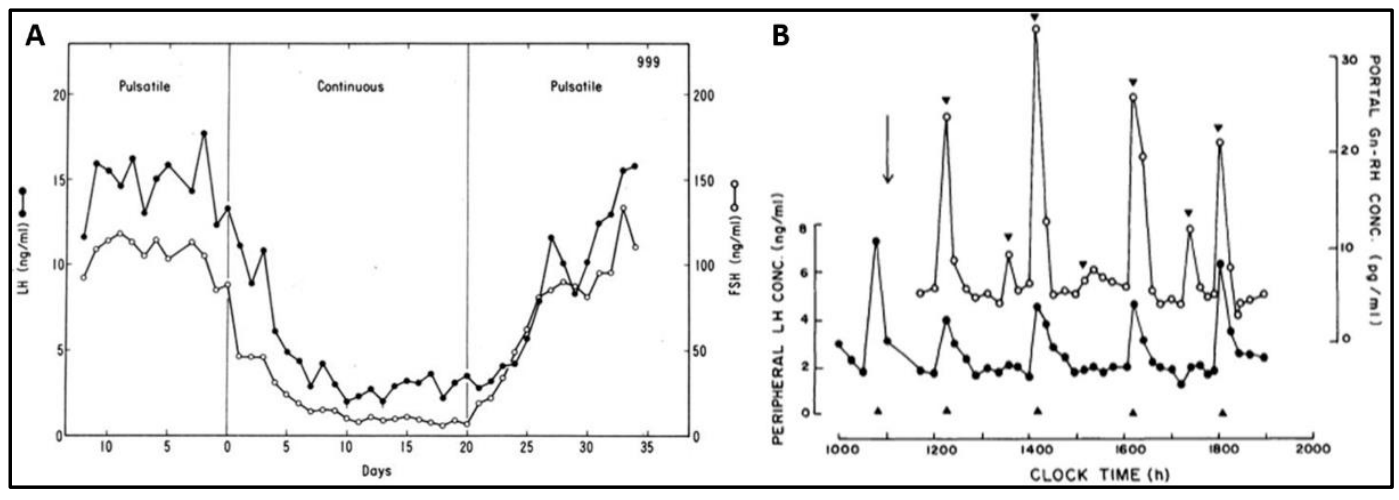

Figure 1-3: Correlation between GnRH and LH pulsatile secretion. Figures adapted from ref. [17, 18] (A) A pulsatile stimulation of GnRH is required to maintain FSH and LH level. The closed symbols indicates LH secretion and open symbols indicates FSH secretion. In the middle block, it shows when the stimulation of GnRH is in a continuous but not pulsatile way, both FSH and LH secretion cannot be maintained. (B) Correlation pulse between GnRH and LH. The record of LH pulses within 10 hours matches the GnRH pulse position.

Later, researchers realised this pulsatile GnRH signal regulates $\mathrm{LH}$ and FSH stimulation by frequency variation. A study using castrate testosterone replace rat as model revealed that FSH stimulation is more sensitive to low-frequency GnRH pulses (less than one pulse in two to three hours) whereas high-frequency GnRH pulses (more than one per hour) tend to release LH [20]. In 2009, Tsutsumi et al. applied fluorescence resonance energy transfer (FRET) reporters in live cells after the GnRH pulse treatment to monitor the dynamic response in the system and utilised two types of immortalised gonadotrope cell termed $\alpha \mathrm{T} 3-1$ and L $\beta$ T2 to study the molecular mechanism for gonadotropin synthesis induced by different GnRH frequency and amplitude.

In his article, he proposed two models:

1) Tonic stimulation of $\mathrm{GnRH}$ and pulsatile stimulation of $\mathrm{GnRH}$ generate different signalling responses in the activation of G-proteins, and this differential activation may provide the molecular basis for frequency and amplitude-dependent signalling by the GnRH receptor (Figure 1-4). 


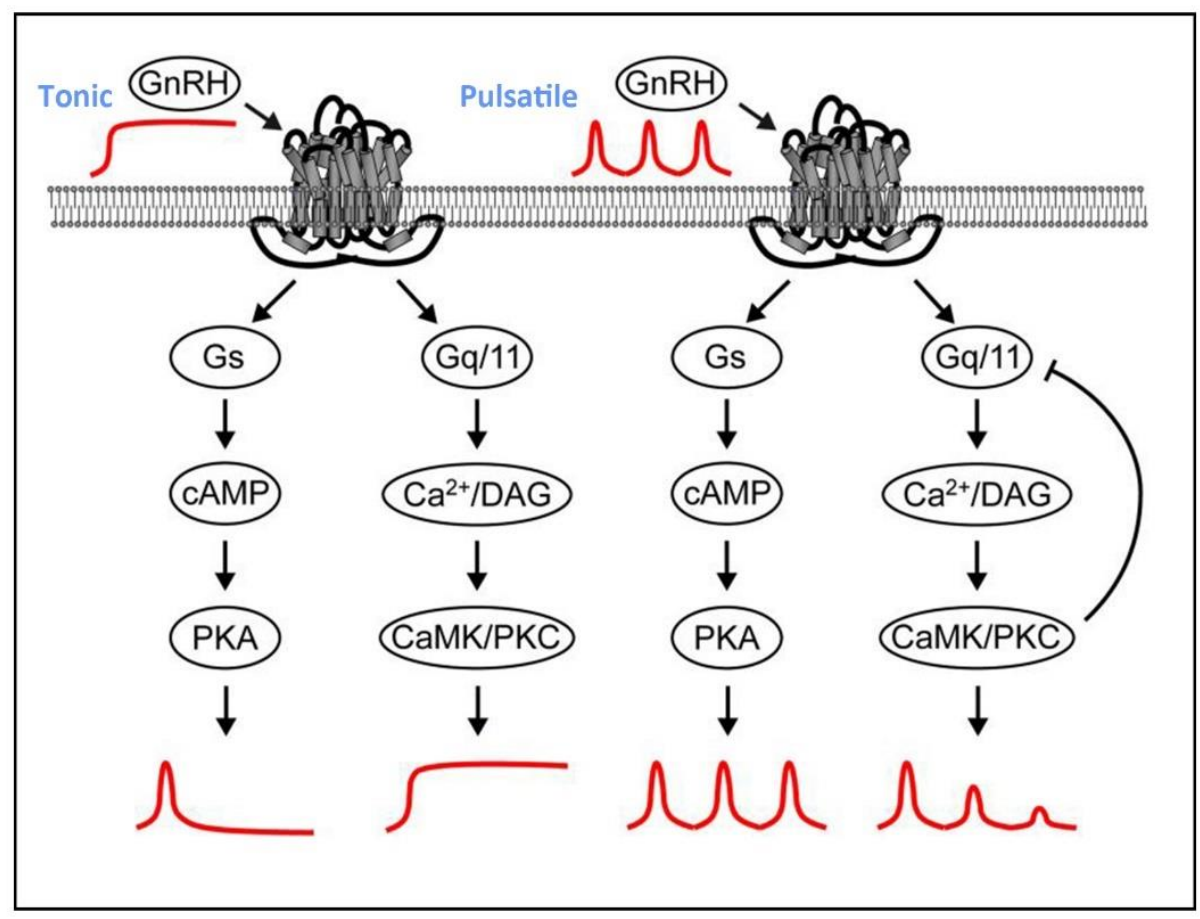

Figure 1-4: Proposed model for regulation of signalling by the GnRH receptor in response to the tonic (left) or pulsatile (right) GnRH. Figures adapted from ref. [21] When the GnRH stimulation is in a tonic manner, Gs/AMP signalling will be triggered to return to a baseline after a pulse, whereas $\mathrm{Gq} / 11 / \mathrm{DAG} / \mathrm{Ca}^{2+}$ signalling will remain elevated during the entire stimulation mode. When the GnRH stimulation is in a pulsatile format, Gs/AMP signalling will present a matched pulsatile manner, whereas $\mathrm{Gq} / 11 / \mathrm{DAG} / \mathrm{Ca}^{2+}$ signalling will first show a matched pulse but then lower and lower frequency pulses will be observed subsequently. Tsutsumi et al. suggested this phenomenon may be evidence of protein kinase $\mathrm{C}$ or $\mathrm{Ca} 2+/$ calmodulin-dependent protein kinase providing negative feedback toward $\mathrm{Gq} / 11$ to desensitise the signalling.

2) For LH synthesis, slow and fast GnRH pulse regulates the activation of LH $\beta$ promoter by Egr1 and NAB-2 signalling. For FSH synthesis, GnRH pulse difference governs transcription factors fos/jun family and TGIF/SnoN corepressors expression thus regulates the activation of FSH $\beta$ promoter (Figure 1-5) [21]. 


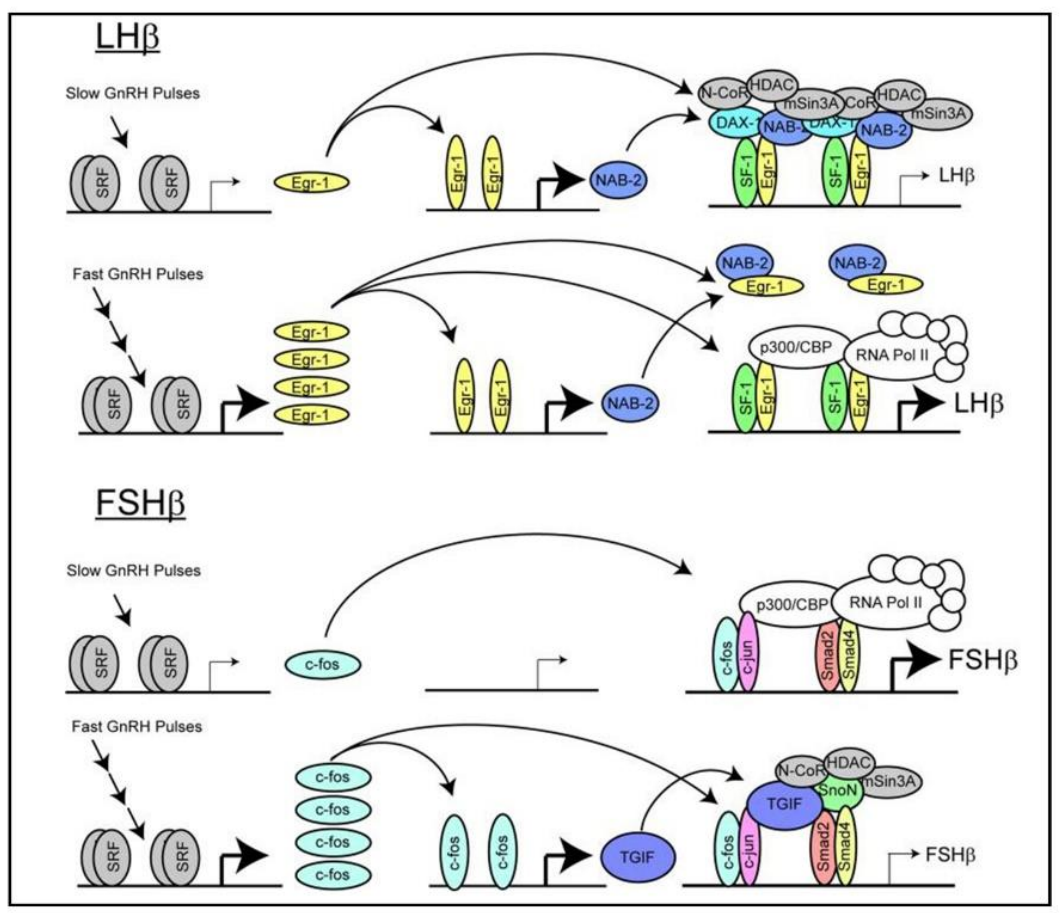

Figure 1-5: Proposed model for pulse regulation of the LHß and FSH及 promoters. Figures adapted from ref. [21]. For the LH synthesis, when it is under slow GnRH pulses (upper first), the Egr1 expression is stimulated transiently and then activated the subsequent increase of NAB-2. NAB-2 and DAX1 thus repress the activation of LH $\beta$ promoter. Under fast GnRH pulses (upper second), the expression of Egr1 is in a sustained high level and quenches NAB-2 activity, thus allows the activation of LH $\beta$ via association with other transcription-activating factors. For the FSH synthesis, when it is under slow GnRH pulses (bottom first), the transient stimulation triggers fos/jun family members expression and then directly activates the LH $\beta$ promoter transcription. However, when it is under fast GnRH pulse frequency, the high level of fos/jun family members enables TGIF/SnoN corepressor which represses the activation of FSH $\beta$ transcription.

\section{GnRH pulse generator and KNDy neurons}

Over half century, with the help of current molecular technology, the hypothesis of the upstream regulation of the "humoral substance" from Harris' has been dramatically expanded. One of the most significant breakthroughs in neuroendocrinology area in the last decade was the discovery of KNDy (kisspeptin/neurokinin B/dynorphin) neurons. In 2003, two groups independently reported that the mutations causing the loss of functions in a G-protein coupled receptor were associated with hypogonadotropic hypogonadism (a medical condition with a decreased level of LH and FSH) [22, 23]. The related ligand of the 
receptor turned out to be a 54-amino acid RFamide related peptide which also known as kisspeptin. It was subsequently found out kisspeptin is a very potent GnRH secretagogue and its neurons locate just above median eminence (where GnRH secreted) in a structure called arcuate nucleus. Later, two peptides one called neurokinin B the other termed dynorphin was found co-expressed with kisspeptin in the kisspeptin neurons in the location of arcuate nucleus [24]. The neuron expressed these three peptides was then coined KNDy neurons [25]. Similar to kisspeptin, neurokinin B can also stimulate GnRH secretion whereas dynorphin was found to be inhibitory to GnRH secretion. Base on these facts, a theoretical model of how KNDy regulating GnRH pulse was then proposed by Goodman et al [26]. They elucidated the GnRH pulsatile release is initiated within the KNDy neuronal network by a reciprocating interplay of stimulatory neurokinin B signals and inhibitory dynorphin inputs (Figure 1-6). The arcuate nucleus where most of the KNDy neurons located was then be considered as the GnRH pulse generator [27, 28]. This finding led to a profound and much-needed revitalisation to the study of GnRH neuroendocrinology and opened a new hormonal therapeutic direction for reproductive treatments, which will be discussed in more details in the next section.

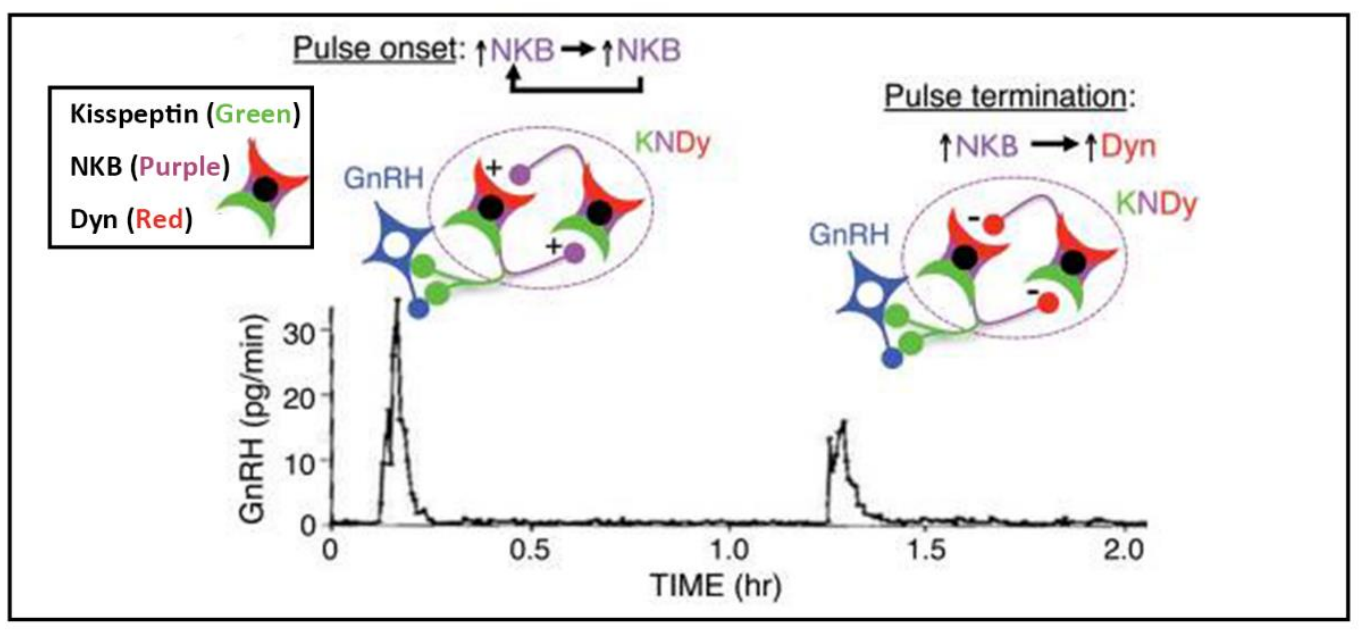

Figure 1-6: The GnRH pulse generator model. Figure adapted from ref. [26]. In this model, they suggest that each pulse of GnRH is triggered by an increase secretion of NKB (purple) from the KNDy neurons (blue) under a positive feedback loop. This loop further stimulates the release of kisspeptin (green) onto GnRH neurons and initiate the GnRH pulse. The termination of the pulse is regulated by Dyn (red), which stimulated by the high level of NKB and it can suppress the Kisspeptin release under a particular time interval. However, The further release of Dyn in the KNDy neurons triggers suppression of Dyn under a negative feedback loop to allow next cycle of NKB releases for subsequent pulse firing. 


\subsubsection{Clinical implications of pulsatile LH signals}

\section{Pulsatile LH as a surrogate marker of GnRH pulsatility}

From the last section, we know that GnRH is the fundamental regulator that controls LH and FSH synthesis by a pulsatile secretion way at the HPG axis. The physiological pulsatile pattern of GnRH secretion (and therefore LH secretion) is tightly regulated by GnRH pulse generator. GnRH's pulsatile frequency-based control feature of LH and FSH release is relevant to the regulation of menstrual cycle (Figure 1-7). During the follicular phase of the ovulatory cycle, GnRH secretes in a manner with high frequency and amplitude. This, in turn, helps LH synthesis and leads to the LH surge which can trigger regular ovulation. After the ovulation, luteinisation of the granulosa cells increases progesterone production which slows GnRH pulse frequency to decrease LH production and increase FSH production to stimulate the next round of ovulation as part of the negative feedback control. Any abnormal GnRH secretion may lead to the dysregulation of LH/FSH secretion and interruption of the menstrual cycle and could result in reproductive disorder.

However, direct measurement of pulsatile hypothalamic marker activity in human remains challenging. Major difficulties including the ethical concerns and technical challenges preclude hypophyseal blood or cerebrospinal fluid sampling for hormone pulsatility measurement in the hypothalamus level [29, 30]. This unobservable feature of some hormone release in the upstream regulation was pointed out in the yellow box of Figure 1-1 A, top. Therefore, measuring GnRH pulsatility in human is not feasible either for clinical application for disease diagnosis or human study to investigate potential therapy. The measurement of LH instead of $\mathrm{GnRH}$ is significant for examining GnRH pulsatility as the correlation between LH/GnRH pulsatile pattern has been well-characterised [31] (Figure 1-3 B). As an indirect indicator but more accessible via circulating blood, LH pulsatility became the best surrogate marker for GnRH pulsatility measurement in many studies $[32,33]$. 


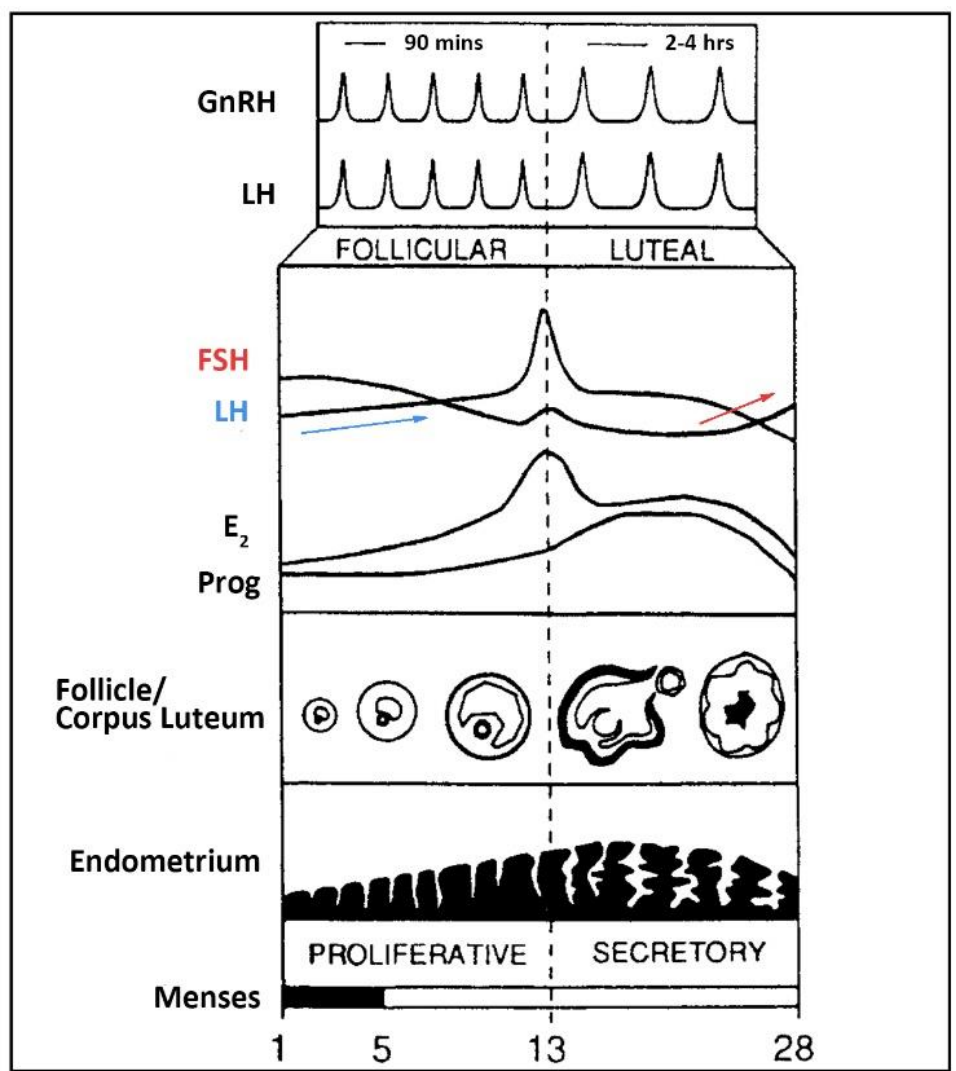

Figure 1-7: GnRH pulsatility and the menstrual cycle. The blue arrow represents the fast GnRH pulsatility frequency favour LH secretion in the Follicular phase, and the red arrow represents the slow GnRH pulsatility frequency favour FSH secretion in the late Luteal phase.

\section{Physiological and pathological implications of LH pulsatility}

In normal healthy individuals, LH pulse occurs about every 90 minutes with a typical blood concentration range about 3 to $10 \mathrm{IU} / \mathrm{L}$. Abnormal LH pulsatility state can be broadly classified as High and Low pulsatility. Figure 1-8 lists some of the physiological and pathological condition associated with these patterns.

High LH pulsatility state represents high LH secretion amplitude and frequency. The LH secretion of female undergoing menopause state is typically in high amplitude and frequency, as the secretion of gonadotropins is secreted unrestrained by the ovarian feedback, this will become more obvious in obese menopause women [34]. Precocious puberty is another condition exhibits high LH pulsatility state because the onset of pubertal maturation is affected by reactivation of GnRH pulse frequency [35]. In an anovulatory patient who diagnoses polycystic ovary 
syndrome (PCOS), their LH pulsatility state also shows in rapid format, as this allows the pituitary synthesis of LH over FSH and contributes to the increased LH concentrations and LH: FSH ratios typical of this disorder [36]. PCOS is also associated with insulin resistance and hyperinsulinaemia $[37,38]$.

One of the major condition associated with low LH pulsatility state is idiopathic hypogonadotropic hypogonadism (IHH). It is a clinical syndrome which often the result of GnRH deficiency [39] especially for males (the male to female ratio is around 4:1 [40]). The functional absence of GnRH secretion from hypothalamic hypophysiotropic neurons or a defect in its action at the level of the gonadotrope in the case of mutations in the GnRH receptor is the cause of this disease. Functional hypogonadotropic hypogonadism (also referred as hypothalamic amenorrhea, HA) is another disease associated with low LH pulsatility in women, but in this condition, most women exhibit high FSH level when compare to LH level [41, 42]. Other conditions include puberty delay [43] and patient with type 2 diabetes [44].

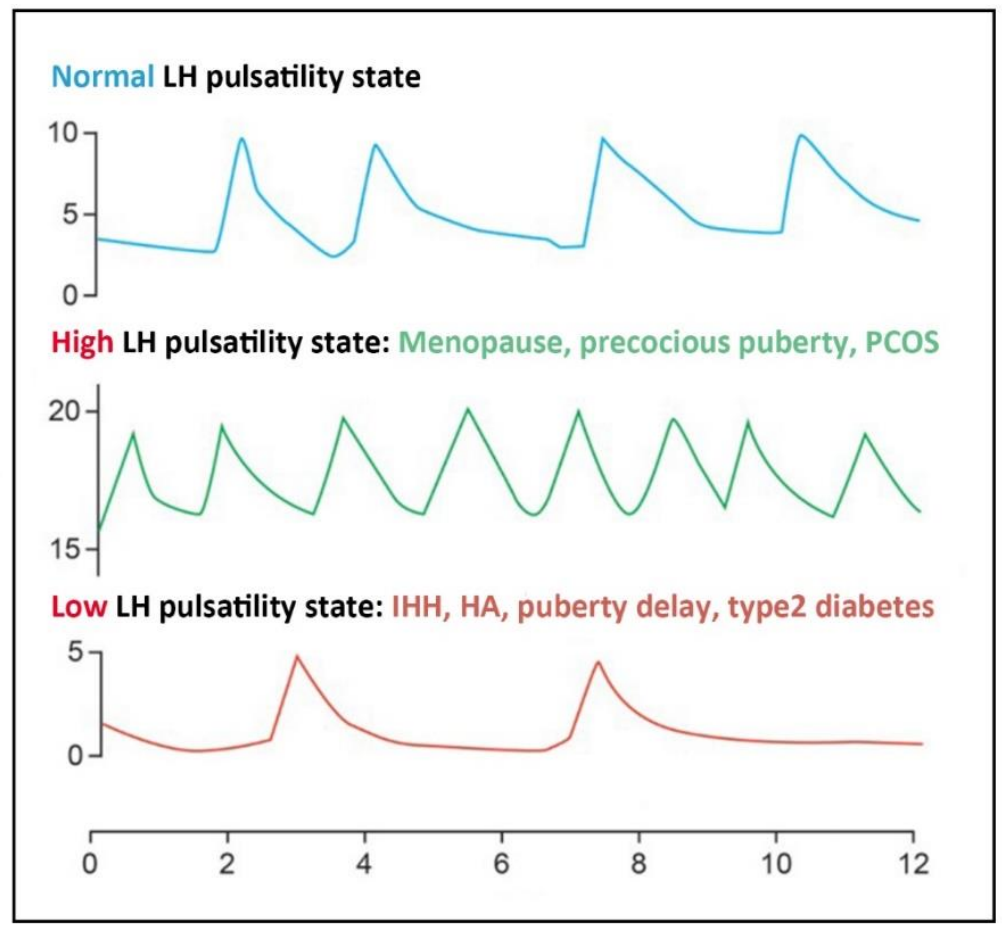

Figure 1-8: Indications for LH pulsatile secretion pattern. Figure adapted from ref. [45]. The blue pattern indicates the normal LH pulsatility state, which shows about 90-110 minutes pulse interval. The green pattern indicates the high LH pulsatility state which normally be observed in the condition such as menopause, precocious puberty and PCOS. 
The red patterns indicates the low LH pulsatility secretion fashion which can be seen in the condition such as IHH, HA, puberty delay and type 2 diabetes.

\section{Potential clinical usage of kisspeptin-based modulation of LH secretion}

Knowing the LH pulsatility state is critical for choosing suitable treatment for hormone-dependent and reproductive diseases described in the previous section. Current hormonal therapies apply GnRH analogues in the clinical practice, they manipulate the HPG axis at the level of GnRH receptor and mainly to suppress the gonadal function for the treating disorders such as endometriosis and uterine fibroids [46, 47]. However, in recent years, the discovery of KNDy neurons offers a novel potential application using kisspeptin (or kisspeptin analogues) for restoring the regular LH pulsatile secretion pattern thus restoring the reproductive function.

For treating diseases present low LH pulsatility state, either using kisspeptin and neurokinin B agonists to enhance the stimulatory tone or applying dynorphin antagonists to suppress the inhibitory tone may have profound therapeutic potential. Regulating the kisspeptin-GPR 54 signalling: in an early human study from Dhillo's group, injection of exogenous kisspeptin was found to be able to stimulate the secretion of LH of a female patient with HA [48]. And later, we further found out that by the repeated administration, injection of kisspeptin can stimulate LH pulsatile secretion in a female patient with HA [49]. However, if the injection of kisspeptin is in a chronic and high dose administration scheme, tachyphylaxis can be induced [50]. Therefore, the injection protocol may need further optimisation for using kisspeptin treatment. In the situation of the male patient, kisspeptin also shows its capability of restoring diminished LH pulsatile secretion in a patient with type 2 diabetes [51]. The response of this LH pulsatility stimulation is comparable to a similar kisspeptin study in healthy male [52]. Regulating the neurokinin $\mathrm{B} /$ neurokinin three receptor (NK3R) signalling: NK3R agonists have been demonstrated in many animal studies capable of accelerating pulsatile LH secretion [53-56]. However, further studies remained to be elucidated for their clinical applications for restoring LH pulsatility in patient. This situation is similar to the investigation of Dynorphin/Kappa Opioid Receptor signalling, whether using Dynorphin antagonists have clinical benefits for treating subfertility remained further investigation.

For treating diseases present high LH pulsatility state, it requires the inhibition

of HPG axis and the antagonists of both Kisspeptin and neurokinin B receptor can 
be used to suppress the stimulatory role of KNDy neurons. Whereas using dynorphin agonist can act as the other way for enhancing the inhibitory action of dynorphin. In a recent study of PCOS patient, Gorkem et al. found out women with PCOS had increased serum kisspeptin levels [57]. As GnRH pulse frequency mainly determines LH but not FSH secretion, slowing GnRH by using kisspeptin antagonist may be able to normalise the relative LH hypersecretion often seen in PCOS, thus promote folliculogenesis and ovulation [58]. One of the most recent studies carried out in Dhillo's group was using an NK3R antagonist for the menopausal hot flush treatment in phase 2 clinical trial [59]. The result shows NK3R antagonist reduced the total weekly number of hot flushes significantly by 45 percentage points. When compared to the profound suppression result by using GnRH analogue administration, the subtler effects of using these antagonists to reduce LH pulsatility seems to be a safer method with fewer side effects [58].

Applying kisspeptin itself has the potential to "fine tune" IVF techniques as the kisspeptin may replace the hCG injection to trigger the LH surge following ovulation induction during the IVF. In Dhillo's group, we performed the first-inhuman study using injection of kisspeptin-54 to trigger egg maturation in women undergoing IVF [60]. Kisspeptin can stimulate a more physiological pattern of gonadotrophin secretion, which may be able to avoid the risk of using hCG, where could induce ovarian hyperstimulation (OHSS). However, further study has to be carried out regarding fully discover the potential advantages and disadvantages comparing to current hCG and other GnRH agonist therapy [61].

A single time measurement of $\mathrm{LH} / \mathrm{FSH}$ is currently using by physicians to obtain information for evaluating infertile female might harbor LH deficiency, as well as LH response to GnRH test [62]. Given the promising result and potential clinical application of using kisspeptin-based modulation of LH secretion, there is an unmet medical need to develop a convenient diagnostic tool that can measure the LH pulsatility - this would lay a foundation for a companion diagnostic for infertility who would specifically benefit from hormonal treatments to restore LH pulsatility and hence reproductive function such as the therapies mentioned above.

In the whole 1.2 section, the importance of knowing the pulsatile LH secretion pattern has been discussed, as the information provided by LH pulsatility offers researchers a way to assess hypothalamic function from the blood. It benefits academic research as researchers are still investigating new signalling pathway 
hypothesis such as KNDy model. From the fertility management angle, knowing the LH pulsatile secretion pattern can also help with infertility treatment as a single measurement of LH is not sufficient enough for the physician to estimate hypothalamic dysfunction.

\subsection{Current methods and challenges for measuring LH pulsatility}

Even though knowing LH pulsatility can provide much useful information both for the therapeutic decision and academic research, there is no feasible clinical way for actual perform the test in the clinic. Most of the biochemistry tests for examination of reproductive function are single point measurement. This format has a limitation because hormone level varies base on the pulsatile release form. And large volume of blood samples is required in a frequently collected way for measuring the whole pulsatility profile. Here in this section, I summarise some of the current methods related to LH pulsatility measurement and point out those key challenges in this area.

\subsubsection{Blood sampling methods}

How to design sampling scheme is critical for identifying LH pulsatile secretion pattern. In the early to mid-1980s, there was a lot of activities focused on the invention of a range of sampling protocols for the measurement of LH pulsatility [63-65]. Due to the complexity of pulsatility analysis, non-standard sampling frequency could result in inappropriate interpretation, especially when random (stochastic) factors are involved [4]. Thus, application of this earlier sampling schemes for LH pulsatility measurement in healthy women at different peroid of the menstrual cycle leading to huge variation of pulse data. For example, identification of LH pulse ranges from 14 to 24 pulses per 24 hours in early follicular phase women, 17 to 29 in late follicular phase women, and lastly from 4 to 16 in mid-luteal phase women [66-69]. After a decade's refinement, the current standard sampling protocol is about every 10 mins over 8 to 12 hours [12].

\subsubsection{Assay methods}

The very first assay for LH was developed by Rayford's group as a radioimmunoassay using hCG antibody [70], which they utilised the cross- 
reactivity of hCG and $\mathrm{LH}$ reported [71]. However, most of the early radioimmunoassay requires few days for assay development. Later in the early $80 \mathrm{~s}$, rapid radioimmunoassay has been developed and the development time shortens to around 3 hours with one hour protein incubation time [72]. But the specificity of these assays remains questionable as the cross-reaction with gonadotropins sharing the same $\alpha$ subunit. It is until the development of monoclonal antibody which capable of recognising different epitopes on the hormone molecule, the crossreactivity issue with $\mathrm{hCG}$, has been solved $[73,74]$. In these sandwich-type assays, two antibodies are developed against two different epitopes of the LH for increasing the specificity. The first capturing antibody is immobilised to a solid phase matrix, and the secondary antibody is conjugated to an enzyme to couple with colourimetric or other optic signal response. It is also called excess reagent assay as these assays do not rely on competition for epitopes between two antibodies. Therefore the excess amount of assay reagent can be added to accelerate the reaction time. The format mentioned is also the base of today's commercialised LH assay.

In most of the clinical lab, automated immunometric assay platform for measuring hormones are available. Platforms such as Roche's Cobas, Siemens centaur and Abbott's architect provide automated hormone assay option [75]. The essential element of this automated platforms is the antibody-based reagent excess assay. Precision timing and software interface control allows the machines to perform different steps efficiently. However, multiple reagents needed, expensive price tag and large footprint limit their use only in high-end hospitals or third-party laboratories (Figure 1-9) [76]. Besides the automated platform, simple manual assay including LH test strip which based on using gold nanoparticle labelling antibody and signal enhancers is available today for single LH measurement, especially for the application on urine LH surge detection for ovulation [77]. 


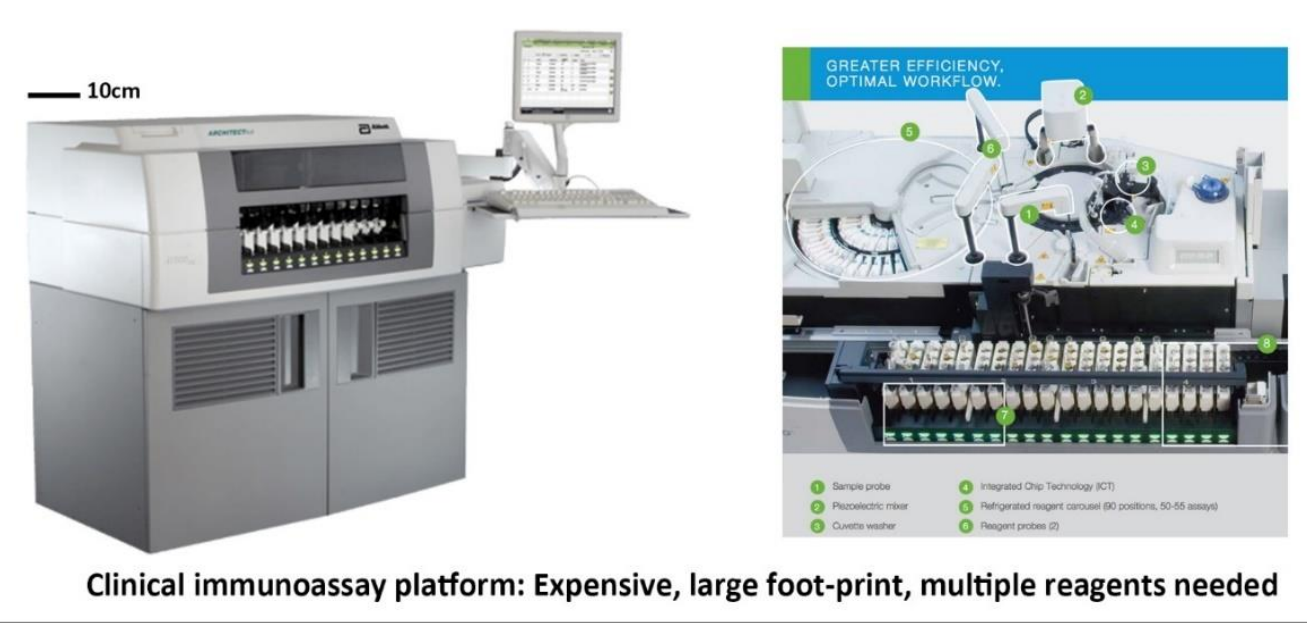

Figure 1-9: Immunoassay platform in the clinics. Abbott's ARCHITECT immunoassay platform. Clinical immunoassay platform can achieve high accuracy, but most of the platforms are expensive and has tremendous size. Fridge storage of some of the assay reagents is required as most of the assay apply antibody-based detection method.

\subsubsection{Analytical methods}

Using the appropriate pulse analysis method is extremely important to avoid false information as the actual data obtained for the measurement may have a lot of "pulse in pulse" due to low signal-to-noise ratio. Before the development of computer-assisted technology, researchers attempted using eye observation and defining the LH pulses based on an increase flowed by a decrease in random concentration units [78]. In early $80 \mathrm{~s}$, thanks to the computer-assisted design, combining defined sampling protocol and pulse detection algorithm for pulsatile hormone analysis became popular and program such as Pulsar [79] and Cycle Detector [80] have been developed based on different mathematical construction. However, the inconsistency of the result generated by analysing same sample set has been pointed out by Evans et al. which they tried to compare the number of LH pulses identified in an LH dataset where concentration-time series over 24 hours of time for normal women by four different early pulse analysis programs. The pulse determined in the same sample set varies from 3 to 17 [81]. Improvement has been made in the late 80s for the development of Detect [82] and Cluster [83] programs. They share the properties of being strictly statistically based and identify the statistically significant increases and decreases in the concentration-time series with different algorisms (such as sliding T-test). 
However, hormone pulse is not only just peak but indeed covers both a secretory event and factors that kinetically affect clearance of the hormone (including association, distribution, metabolism and excretion). Base on this, deconvolution method was then proposing by researchers for LH pulsatility analysis. In mathematics, deconvolution refers the process which applied to reverse the effects of the convolution of the measured data [84]. In the early mathematic models raised by few academic groups, hormone kinetic information such as estimated LH halflife was required [85]. It limits some of the applications as prior information is needed for every pulsatility measurement.

To solve this, Veldhuis et al. developed Deconv algorism which allows simultaneous assessment of both the characteristics of the secretory hormone burst and provided an estimate of the hormone half-life [86]. This multiparameter deconvolution method differed from earlier deconvolution procedures in that it describes secretion as a variable number of Gaussian-shaped secretion events convolved with a one- or two-component exponential elimination and then fit directly to the concentration-time series. In contrast, Deconva allows for the identification of significant secretion events directly by the fitting process. Figure 1-10 shows a LH concentration-time series obtained from a normal woman during the early follicular phase of her menstrual cycle (top) and the LH secretory events as resolved by application of Deconv (bottom). This method has been widely applied in many later studies, some of them with slight modification such as the development of Autodecon in 2008 [87]. In recent years, Bayesian analysis approach appears to be an alternative method for deconvolution analysis, especially in the situation of low signal-to-noise ratio [88]. 


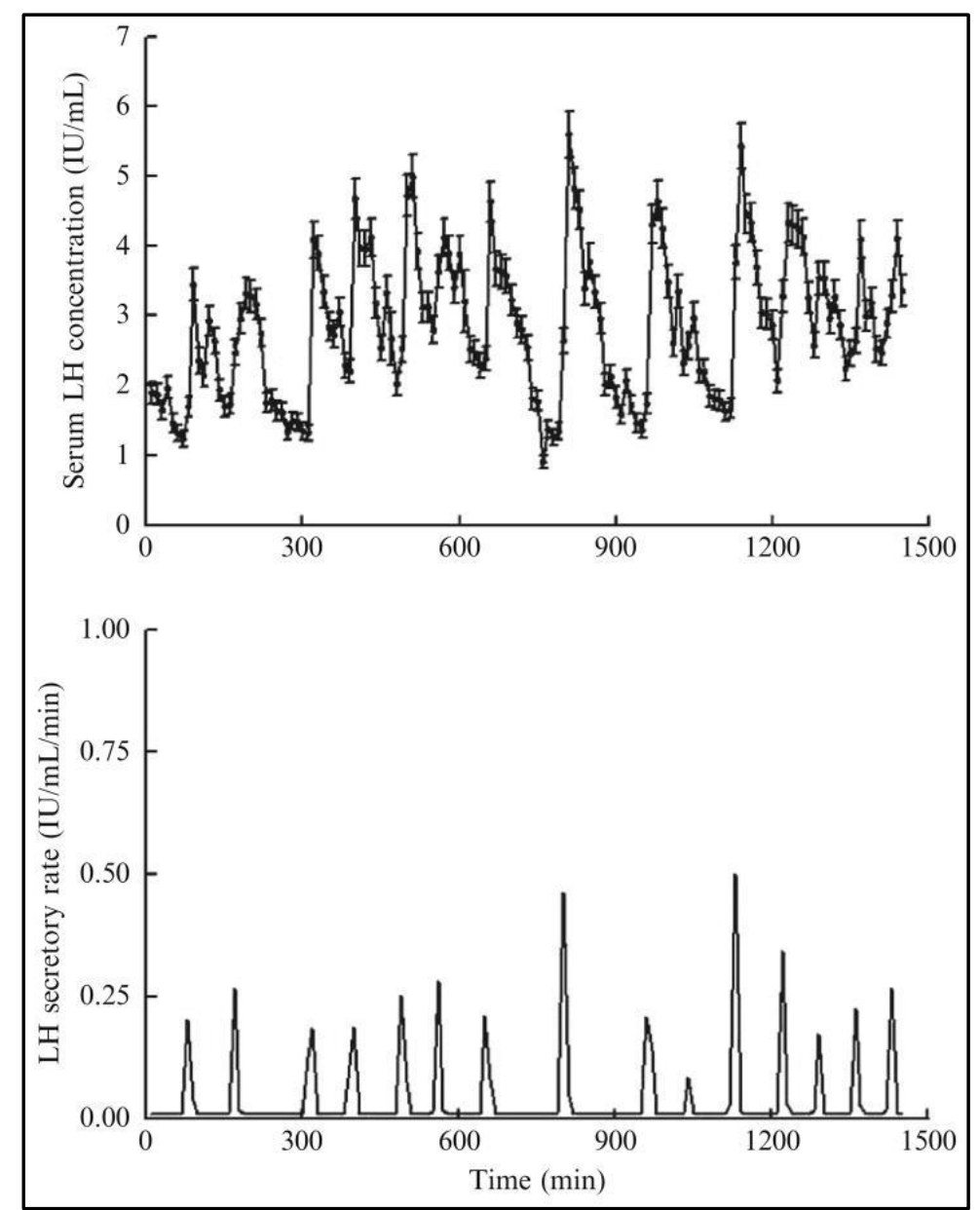

Figure 1-10: Deconvolution analysis. Figure adapted from ref. [86] (Top) A serum LH concentration-time series constructed from samples obtained from a normal woman in the early follicular phase of her menstrual cycle. Samples were collected at 10-min intervals over a 24-h period. (Bottom) LH secretory bursts resolved from this concentration-time series using the deconvolution procedure Deconv

\subsubsection{Challenges}

Overall, the remaining challenges can be summarised into points below:

1) LH pulsatility resolution is restricted by the sampling protocol and antibody assay limitation. There is no current method can real-time monitoring $\mathrm{LH}$ pulsatility.

2) Even though automated LH assay is commonly used in the clinical setting, it is costly to perform the test at multiple time points. 
3) Pulse analysis remains challenging as it requires complicated algorism for deconvolution.

Therefore, I want to propose novel method base on aptamer-enble sensor to address some of these challenges.

\subsection{Aptamer-enable sensor for LH pulsatility measurement}

\subsubsection{Aptamer and SELEX}

Aptamers are short DNA or RNA oligonucleotides that have a defined avidity and specificity for their chosen target ligands. Their targets include proteins, cells, pathogens and even small molecules. Since the first reports in the early 1990s [89, 90], "the aptamer technology" has been expanded to different areas such as diagnostic and therapeutic [91, 92]. The single strand nature of aptamer enables them to fold into defined 3D motifs which have specific binding properties to the target ligand by hydrogen binding, charge-charge interactions and van der Waal forces. The first aptamer was in RNA form, and later ssDNA was developed. RNA aptamer invariably harbours stronger affinity and specificity properties as the RNA has a greater variety to stable secondary structure formation. However, RNA aptamers lack chemical stability in alkali conditions and can be quickly degraded in environment containing RNAase enzymes, therefore in diagnostic or environmental application, people tend to use DNA rather than RNA aptamers as the affinity element for the integration of different sensing platform due to the robustness and stableness nature of DNA aptamer [92]. In recent years, aptamers consist of non-natural components such as alternative nucleobases, sugars, or a connecting backbone with a different chemical structure, termed XNA, has also been developed [93].

Both DNA and RNA aptamers are generated from large random sequence libraries through an in vitro evolution process termed Systematic Evolution of Ligands by Exponential Evolution (SELEX) [94]. Begins with an incubation of a large synthetic pool containing 1013 1015 oligonucleotides and a certain among of targets, the whole SELEX is then followed a cyclic process (Figure 1-11): 1) 
Separation of the unbound sequences from the ssDNA-protein bound-complex; 2) Harvest and amplify the remained ssDNA by PCR; 3) Isolation of the singlestranded sequences from double-strand PCR products; 4) Incubate the enriched pool with the target again. After 10 20 round, aptamers which can bind to the target with high affinity and specificity can be generated. The inception of SELEX was in 1990 since then many alternative selection methods have been developed including magnetic bead-based SELEX, Cell SELEX, Capture-SELEX, etc. While methods may vary the procedure is generally the same across all the techniques, the basic SELEX procedure is illustrated. Among all these methods, nitrocellulose membrane filtration is one of the most common used method for label-free protein targets. Originally this method was developed in 1968 by Pristoupil and Kramlova as a means to separate RNA from proteins [95]. In our lab, using classic aptamer selection methods such as magnetic bead and membrane, we developed multiple aptamers for different disease targets including pfLDH [96], HECT ubiquitin ligase WWP1 [97], SARS coronavirus helicase [98] and Mycobacterium tuberculosis polyphosphate kinase 2 [99].

Aptamer has initially been developed as therapeutic agents. The most wellknown example is the FDA-approved aptamer drug "Pegaptanib", which is an aptamer-based therapeutic agent used to treat the wet form of age-related macular degeneration [100]. However, there are still many limitations for aptamer-based therapeutics, such as their poor pharmacokinetic properties, as well as the unmodified aptamers are challenging to penetrate cell membrane [101]. Nowadays, more and more research groups focus on using aptamer as the binding molecule for the development of biosensing system, especially for the DNA aptamers. The synthesis, cost and robustness make aptamer an excellent binding agent for a diagnostic device to sense different biomarkers. Biosensors that apply aptamers as the core affinity element called aptasensor. The biggest challenge for these aptamerbased biosensors is how to design the best way to change the binding event between the aptamer and its target into a quantifiable readout signal. Thanks to the flexibility of aptamer modification, a wide range of strategies of design can be applied to the development of these aptasensors [102]. Reporter modified aptamers can act as a capturing probe for the target and transduce into either optic/florescent signals or electrochemical signals. I only focus on the electrochemical-based aptasensor in this section. 


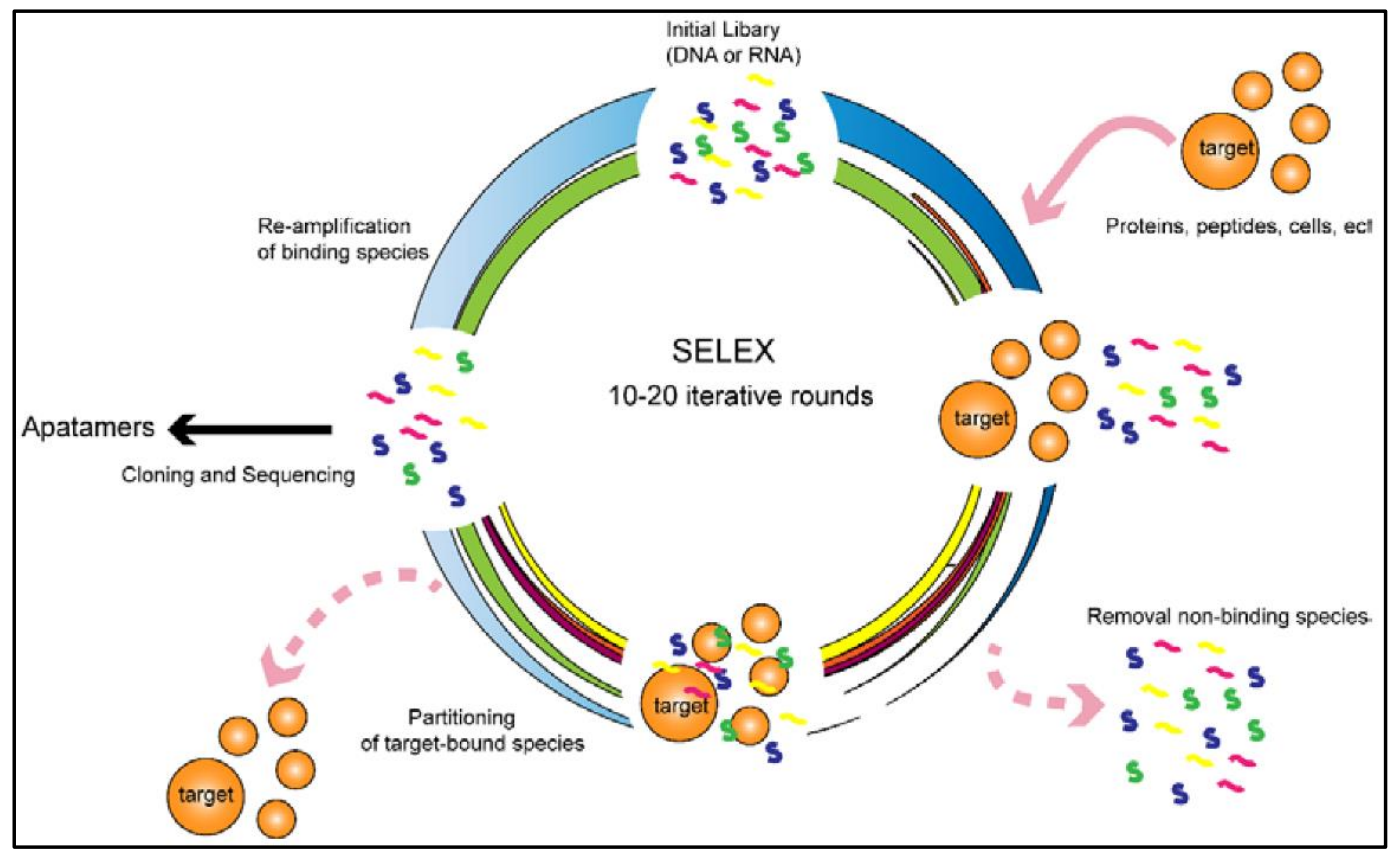

Figure 1-11: SELEX steps. Figure adapted from ref. [103] Normal SELEX procedure including the (1) separation of the unbound sequences from the ssDNA-protein boundcomplex; (2) harvest and amplify the remained ssDNA by PCR; (3) isolation of the singlestranded sequences from double-strand PCR products; (4) incubate the enriched pool with the target again. After 10 20 round, aptamers which can bind to the target with high affinity and specificity can be generated.

\subsubsection{Electrochemical-based aptasensor}

Electrochemical-based aptasensors (E-AB) are one of the most well-developed aptasensor types. They are aptamers modified with electrochemical reporters which can transduce the binding event into electrochemical signals either in a current or impedance format. These signals can also be applied by attaching bio-catalytic labels. Figure 1-12 A illustrates the basic construction of E-Ab which consists of the target, the bioreceptor aptamer (can be modified with redox reporters), the transducer electrode and the electrochemical response signal readout. Figure 1-12 B to $\mathbf{F}$ illustrate the major E-AB formats. The first E-AB was reported in 2005 as a sandwich sensor detects by amperometry based on the glucose dehydrogenaselabelled signaling system [104]. Sharing the similarity of well-established ELISA (enzyme-linked immunosorbent assay), sandwich type of E-AB (Figure 1-12 F) requires a sandwich recognition mechanism. Electrode-bound aptamer is used to capture the target ligands together with some redox-active species to the electrode. For example, an electrochemical assay for thrombin detection was developed by 
applying CdS conjugated gold nanoparticles and electrode-bound thrombin aptamer as a sandwich format. In this E-AB, a primary aptamer was used as the capturing probe to immobilise target, and a secondary reporter aptamer which conjugated with CdS coated gold nanoparticle [105]. Another example is the application of aptamer-conjugated silver nanoparticles for the detection of Staphylococcus aureus, which also applied an electrochemical dual-aptamer-based sandwich format for the target detection [106]. Most of the sandwich type E-Abs share the similarity of using aptamers pairs. However, these sensors have limited application since not that many aptamer pairs have been developed and also, similar to ELISA, these assay format still require multiple reagent addition steps.

Another E-AB type based on impedance spectroscopy. This sensing system applies aptamer-coated electrode and flowing reversible redox couple reagents such as Ferrocene and ferrocyanides to detect the change of interfacial electron-transfer resistance after the binding of aptamer and targets occurs. Impedance spectroscopy based E-AB was reported to have femtomolar detection limit against [107]. One of the advantages for this type of E-AB compared to the sandwich type E-AB is that it is label-free and relatively easy to regenerate as there no need to worry about harsh regeneration trigger reporters' degradation. However, this type of sensor still requires adding the redox couple reagent onto the sensor.

Distinguish from impedance-based E-AB, E-AB with intrinsic redox reporter modification seems to be more common used in the recent years. In this type of E$\mathrm{AB}$, a conformational change is required. Upon binding triggered conformational change, the distance between the reporters on aptamer to the sensor surface (typically gold electrode) will change. Thus the electron transfer rate of the reporter will change. This signal change can be quantified by different electrochemical techniques such as Square Wave Voltammetry, AC voltammetry, etc. [108]. Depends on the electron transfer rate change, these sensors can either be in a "signal on" format or "signal off" format [109]. As the most widely developed sensing platform for aptamer sensor, this type of $\mathrm{E}-\mathrm{AB}$ has been developed against targets from proteins to small molecules. Continuous sensing-based on this system has been developed by Plaxco group on drug pharmacokinetic monitoring [110]. 


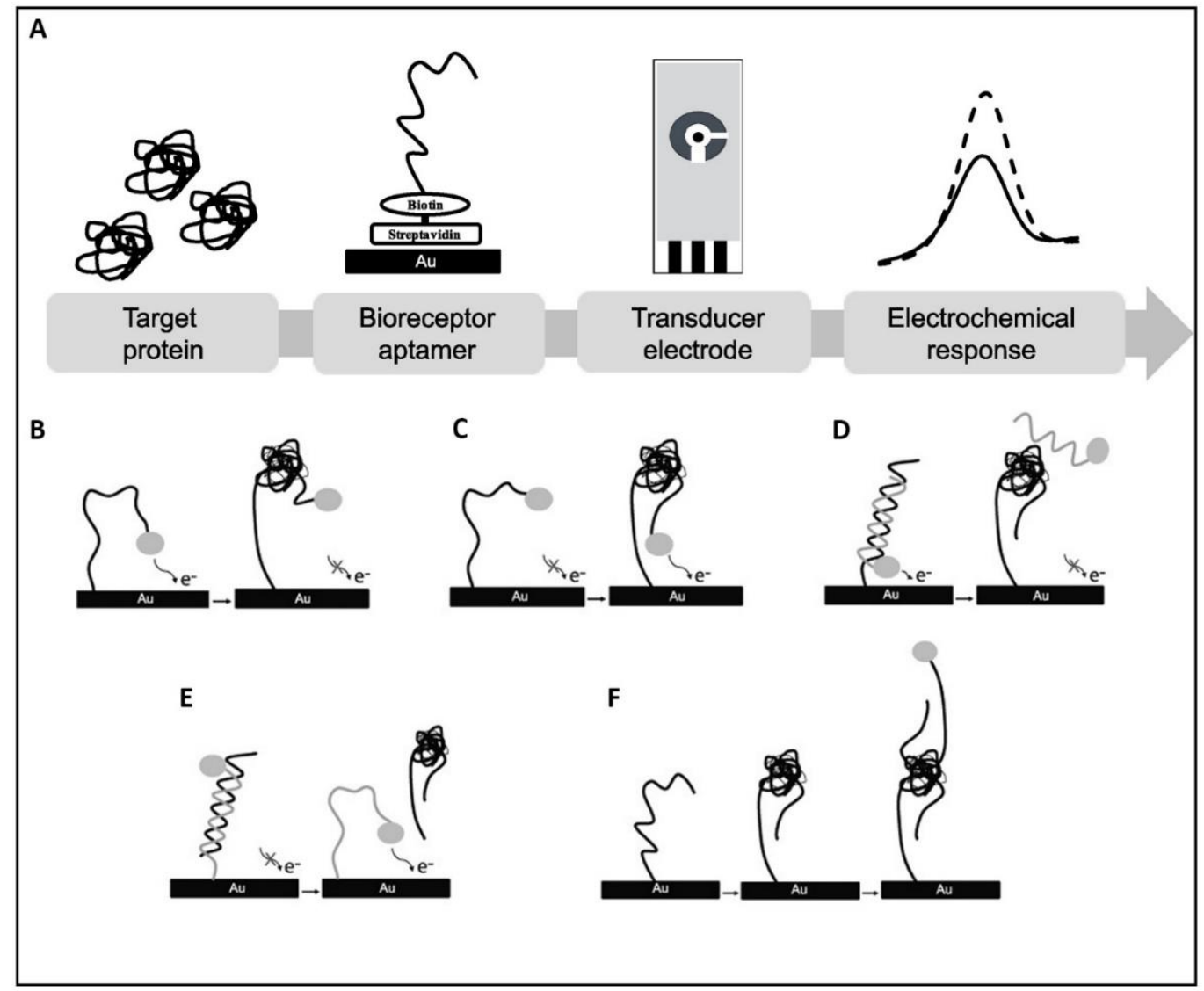

Figure 1-12: Electrochemical aptasensor design. Figure adapted from ref. [111] (A) Illustration of the major components in E-AB. (B-F) Different designs of E-AB sensing format, including conformational change signal on and signal off sensors, target displacement sensors, and sandwich type sensor.

\subsubsection{Advantage of using E-AB platform for LH pulsatility measurement}

Using the E-AB technology, the first two challenges of LH pulsatility measurement summarized in section 1.3.4 may be solved. First, E-AB platform potentially can measure LH in a continuous format instead of single point measurement. In this case the refection of the pulsatility is in real-time format with higher time resolution. Second, E-AB platform requires is a one-step reaction, it does not require multiple reagents for signal development, which provides a possible easier way to reduce cost and adapt to automatic assay format. In this thesis, I tried few different assay formats to fulfill these requirements including continuous sensing platform, automatic 96 well plate platform. 


\subsection{Aims of study}

This thesis aims to develop an entirely novel sensing system for LH pulsatility measurement and evaluate the system via pilot clinical trials. Iwant to address some of the current challenges of LH pulsatility measurement:

1) Large volume of the blood sample is required for the immunological assay.

2) Multiple steps with a series of additional reagents are required in the automatic immunological assay, which increased the measurement cost.

3) No continuous sensing format is available to monitor LH pulsatility in a realtime format.

Therefore, I aim to:

1) Develop a novel human LH aptamer with strong binding affinity and specificity.

2) Develop the electrochemical assay using this aptamer and shrink down the required sample volume for $\mathrm{LH}$ detection.

3) Develop different device prototypes which can be applied for pilot study in the clinics including automatic aptamer-based platform and continuous sensing platform.

4) Evaluate the best device prototype using actual patient samples with different LH pulsatility profiles.

\subsection{Scope of thesis}

What I have discussed in Chapter 1 is the motivation driving why I want to develop device for LH pulsatility. I answered:

1) What is the physiology basis of $\mathrm{GnRH} / \mathrm{LH}$ pulsatile secretion;

2) Why measuring the LH pulsatility is significant;

3) How other people did it from history and what are the remaining challenges; 
4) What I propose to address these challenges.

In Chapter 2 to 4, I further elucidate:

5) How I develop solutions to address these challenges by:

Chapter 2: Generate the molecular binding element against LH and characterise its binding affinity, specificity, binding kinetics and conformational changing dynamics via aptamer technology;

Chapter 3: Modify the molecule with an electrochemical reporter and develop electrochemical assay method for the LH quantification

Chapter 4: Minimize the electrochemical assay and prototype different device designs and establish an optimal format for the clinical application.

In Chapter 5, I examine the proposed solution via clinical samples and answer whether I meet the original aim.

In Chapter 6, I describe the overall contribution of this project and the potential future directions. 


\section{CHAPTER 2 | Generation of LH specific aptamer}

In this chapter, I focus on describing how I generate and characterise the core binding element in the sensing system - the LH specific aptamer. First, I used a nitrocellulose-membrane-based SELEX method to generate single-stranded DNA aptamers against LH. Second, after picking the selected candidates analysed by Sanger Sequencing, the affinity, specificity, binding kinetics and conformational change dynamics of these LH binding sequences were well characterised by biophysical and biochemical assays. I demonstrate that the key aptamer I generated has high binding affinity $\left(\mathrm{K}_{\mathrm{D}}=300 \mathrm{nM}\right)$ against $\mathrm{LH}$ but not recognising $\mathrm{LH}$ structural similar proteins such as FSH and TSH. The dissociation rate of the aptamer estimated by Surface Plasmon Resonance is relatively fast $\left(\mathrm{t}_{1 / 2}<15 \mathrm{mins}\right)$ - this feature, together with the conformational change upon LH binding confirmed via Circular Dichroism, benefit the further design of the continuously structure switching sensing system.

Keywords: SELEX, ELONA, Surface Plasmon Resonance, Circular Dichroism

\subsection{Background}

In this chapter, I aim at generating an LH specific aptamer and adapt this aptamer to the E-AB sensing format mentioned in the previous section. To fulfil this goal, the aptamer candidate should meet 3 of the major requirements (Figure 2-1):

1) Strong binding affinity against $\mathrm{LH}$ and no significant interference with $\mathrm{LH}$ structural similar proteins such as FSH and TSH;

2) Upon binding the $\mathrm{LH}$, the candidate should trigger a certain degree of conformational change so that it can be further modified based on the structureswitching electrochemical sensing scheme;

3) Preferably, the candidate would have a relatively fast off-rate so that the sensing response can be recorded in a real-time format;

To justify whether the selected aptamer is suitable for the further sensing design, biophysical and biochemical techniques are required for the characterisation of the 
selected aptamer candidate's properties. For the affinity characterisation, biophysical methods such as Surface Plasmon Resonance (SPR) [112], Isothermal Titration Calorimetry (ITC) [113], Microscale thermophoresis (MST) [114] are commonly used for aptamer-target affinity characterisation. One of the advantages of using SPR for aptamer affinity characterisation for this project is that the assay is in a continuous flow format, which is similar to what I want to achieve using the $\mathrm{E}-\mathrm{AB}$ form. And by measuring the SPR response, the binding kinetic information can also be obtained from fitting the response to a binding model.

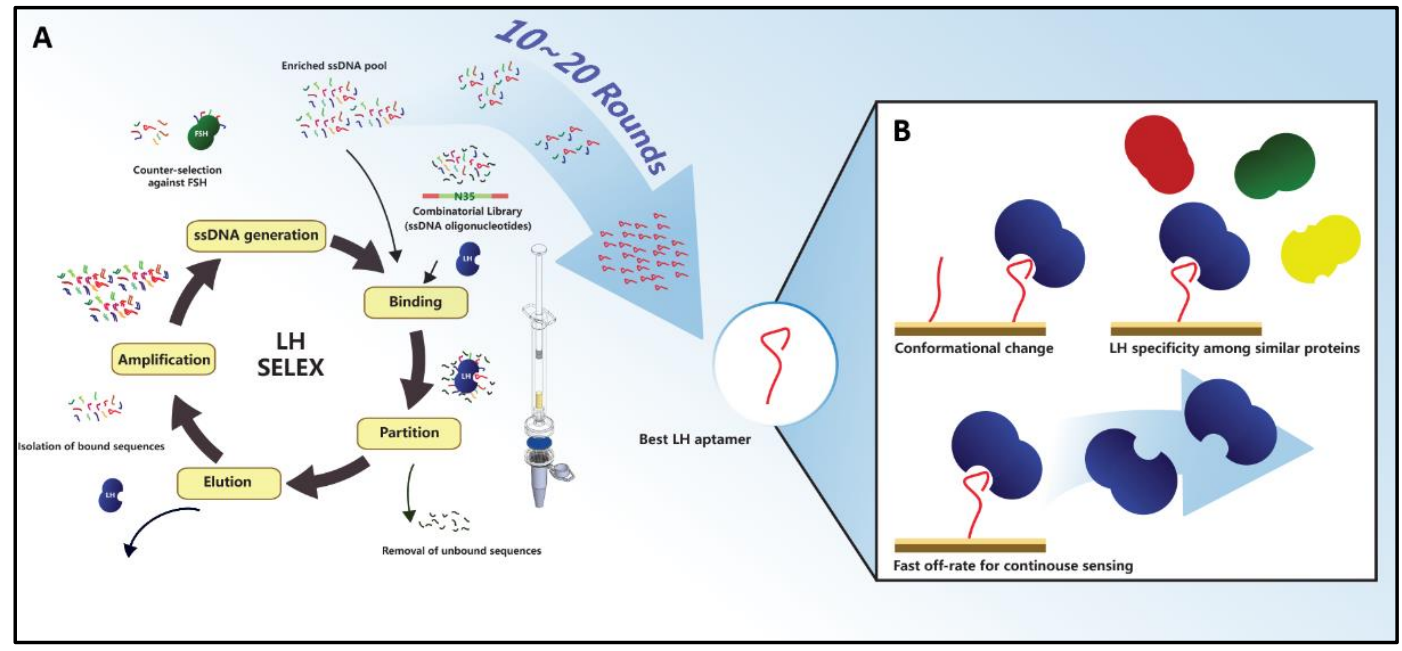

Figure 2-1: LH SELEX and the expected properties of the aptamer. (A) Illustration of the SELEX procedure. Major parts of the in vitro selection cycle are Binding, Partition, Elution, Amplification and single DNA pool generation. (B) Illustration of the 3 requirements I like to get for the new selected LH aptamer. I want to select aptamer which can bind to LH specificity with no/limited cross-reaction with other proteins. A high degree of conformational change upon binding and fast off rate is also perferable as these properties can benefit to the continuous sensing format.

\subsection{Aptamer selection strategy}

A 71-nt single-stranded DNA (ssDNA) library N35 was used as the library for the SELEX. This N35 library consisted of a randomised 35 nucleotides region in the middle, and flanked with two fixed primer binding regions which required for PCR amplification. The primers used for the amplification step are an unmodified forward sequence and a 5'-biotin labelled reverse sequence (Figure 2-2 A). Amplification with these primers can generate double-stranded DNAs with biotin label, which then streptavidin-coated magnetic bead method can be applied for 
generating new ssDNA pool from separating the double-stranded PCR product to signal-stranded DNA.

As there is no affinity tag in the recombinant $\mathrm{LH}$, a nitrocellulose filtration method was designed for the partition step. Nitrocellulose membrane can nonspecifically absorb protein but not nucleic acids. Therefore it can be applied in the separation step during the SELEX. After incubation of the N35 with LH in the binding buffer, the solution was then filtered through nitrocellulose membrane via a syringe setup (Figure 2-2 B, left). By applying the design, the unbound ssDNA can be removed by filtration as the bound ssDNA-LH complex will be absorbed in the nitrocellulose membrane due to hydrophobic interaction and only be eluted by placing the membrane in hot water. Same step was also applied for the counter selection steps against FSH, an LH structural similar protein but instead collecting the ssDNA eluted from the membrane. The ssDNA in the filtrate was then collected for the next selection round. (Figure 2-2 B, right).

Figure 2-2 C, up shows the agarose gel electrophoresis image for the PCR amplification steps of the $1^{\text {st }}, 4^{\text {th }}, 8^{\text {th }}, 12^{\text {th }}, 16^{\text {th }}, 18^{\text {th }}$ and $20^{\text {th }}$ Round ssDNA pool. The 71-nt band in the left lane of all the images shows the evidence of successful recovery of selected sequences in each selection round. Worth noted that nonspecific amplicons could be observed with an increasing trend from $4^{\text {th }}$ round until the Library to Target ratio was reduced from 100:1 to 50:1at $16^{\text {th }}$ round. This phenomenon may occur due to the enrichment of the library as the more template presented in the PCR solution, the easier to generate non-specific amplicons. Oncethe Library to Target ratio is reduced, the selection stringency will be increased. Therefore less template can be obtained after the incubation step. The observation of another increasing trend of non-specific amplicons from $16^{\text {th }}$ to $20^{\text {th }}$ round supports this assumption. Figure 2-2 C, down shows the gel image for the PCR amplification steps of the $1^{\text {st }}$ and $2^{\text {nd }}$ counter selection rounds against FSH. The $1^{\text {st }}$ counter selection round was performed after the $3^{\text {rd }} \mathrm{LH}$ selection round. In the $1^{\text {st }}$ counter selection round, nearly same level of intensity of DNA signal was observed for all the LH bound fraction (black arrow) and FSH bound fraction (red arrow), whereas no FSH bound fraction (blue arrow) can be seen in the $2^{\text {nd }}$ counter selection round, which was performed after $10^{\text {th }} \mathrm{LH}$ selection round.

Overall,20 selection rounds against LH and two counter selection rounds against FSH were conducted by using the nitrocellulose membrane-based method. Apre- 
selection step before the $1^{\text {st }}$ selection round was also conducted via filtering the first library by the membrane and use the filtrate as the start library for LH selection. The purpose of this pre-selection step is to avoid picking up membrane binder sequences.
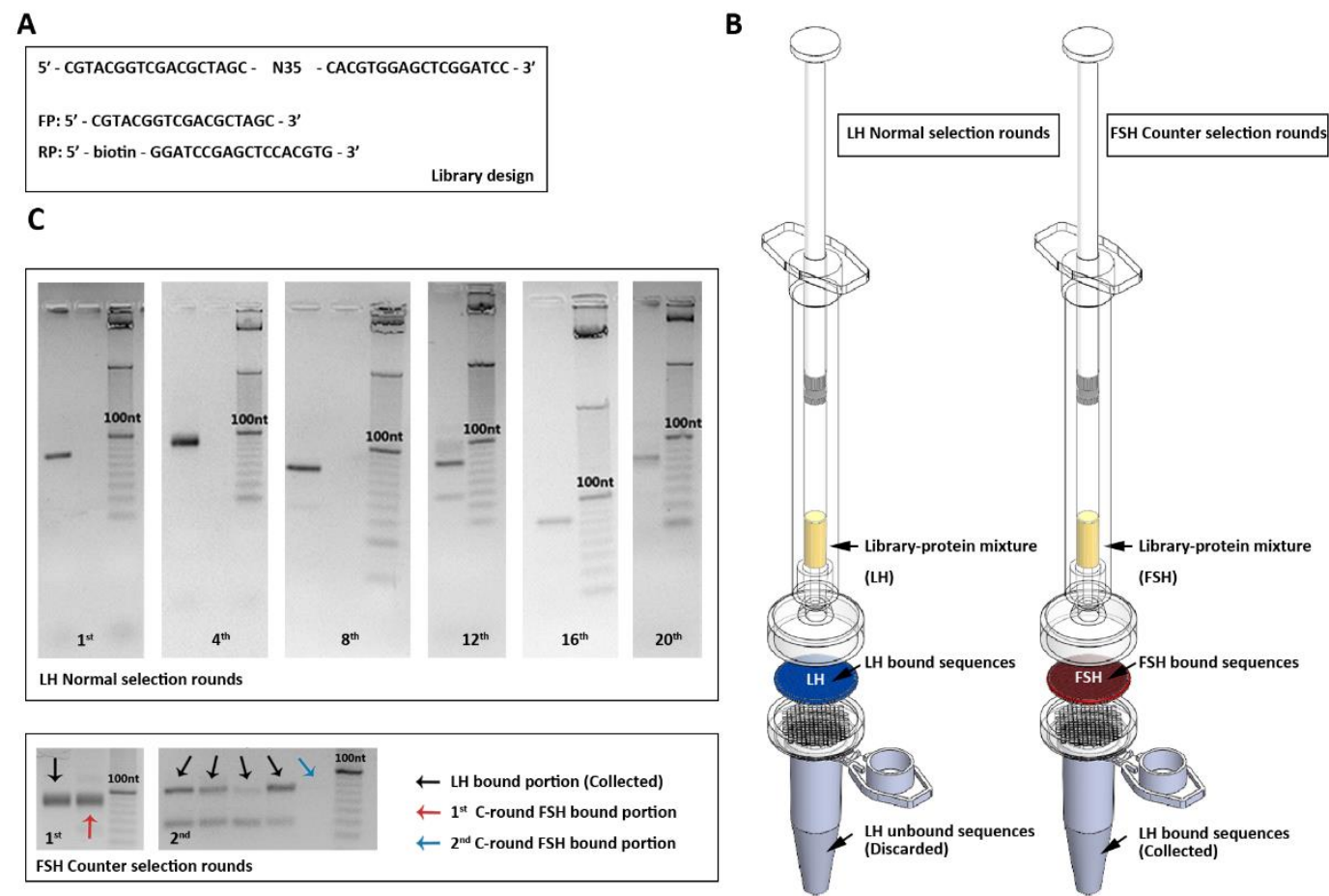

Figure 2-2: Nitrocellulose membrane-based SELEX. (A) Library and primers design. N35 represents the random region of the 35-nt long sequence. (B) Illustration of the membrane-based partition method in normal selection and counter-selection rounds. Yellow colour represents solution mixture of library with LH in the left-hand side and library with FSH in the right-hand side. During the filtration, protein-bound sequences will be absorbed in the membrane (blue LH, red FSH). (C) Agarose gel electrophoresis images obtained from the PCR product of different rounds of selection. The upper figure is the normal selection round from $1^{\text {st }}$ to $20^{\text {th }}$. Bands on the left-hand-side with stronger intensity are the selected library in each round. Non-specific amplicons can be seen in few different rounds with a particular "increase-decrease-increase" trend. Figures on the lower side are counter selection rounds. The four black arrows in the $2^{\text {nd }} \mathrm{FSH}$ counter selection round represent four different fractions collected from the filtrate. 


\subsection{Library enrichment}

\subsubsection{LH binding enrichment}

Enzyme Linked OligoNucleotide Assay (ELONA) was used to monitor the affinity enrichment during the selection process in different selection round. Similar to Enzyme-Linked ImmunoSorbent Assay (ELISA), ELONA is a sandwich-based assay, LH was immobilised onto a high binding 96-well plate and titrated low, middle and high concentration of different round selected ssDNA libraries with biotin tag. These libraries were biotinylated via PCR using biotinylated forward primer and phosphorylated reverse primer (for generation of ssDNA after PCR via Lambda Exonuclease Digestion). The binding signal can be obtained colourimetrically via adding streptavidin HRP and TMB (Figure 2-3 A). Figure 2$3 \mathbf{B}$ shows the result of the binding signal generated by $3^{\text {rd }}, 7^{\text {th }}, 15^{\text {th }}$ and $20^{\text {th }}$ round library. An increasing trend of the affinity can be observed from $3^{\text {rd }}$ to $20^{\text {th }}$ round library. I also incubated the $15^{\text {th }}$ and $20^{\text {th }}$ libraries with FSH to check whether the counter selections were successful or not. The result shows no apparent binding for FSH in both selection round libraries.

A

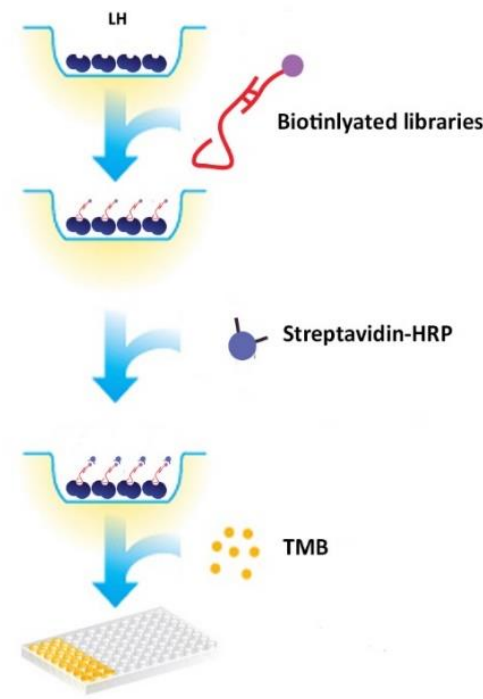

B

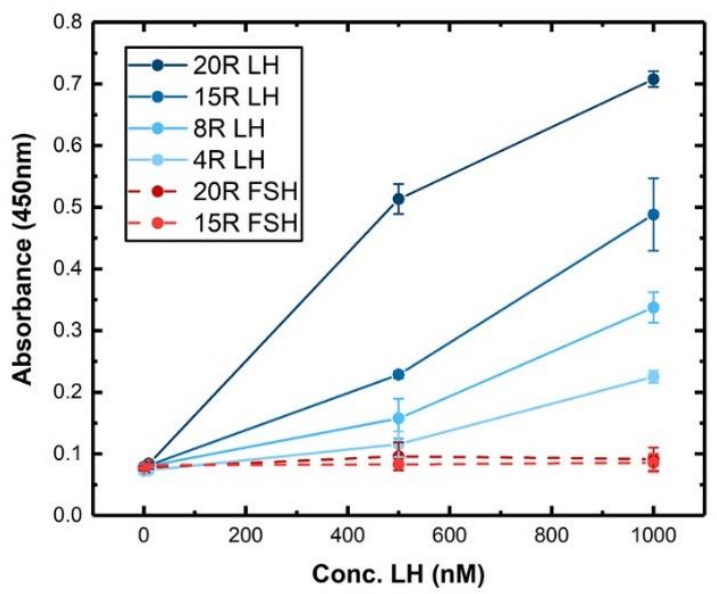

Figure 2-3: ELONA study for affinity enrichment. (A) ELONA principle. LH was coated on the wells, and different concentration of biotinylated ssDNA libraries was added for titration. After adding streptavidin-HRP and TMB, the colorimetric signal can be generated for qualification (B) Colorimetric response of different round libraries from the ELONA. Four blue lines represent libraries from different selection round against LH. Two 
red dash lines represent the negative control of signal response for $20^{\text {th }}$ and $15^{\text {th }}$ round libraries against FSH.

\subsubsection{Further affinity and specificity characterisation of $20 \mathrm{R}$ library}

Electrophoretic Mobility Shift Assay (EMSA) was performed to further estimate the rough binding affinity of the latest selection round library (20R) against LH and check whether this library also binds FSH. The basic principle of the EMSA design is an electrophoretic separation of protein-ssDNA library mixture on polyacrylamide gel for a short period. The stronger protein-ssDNA interaction is, the harder ssDNA separates apart further. Therefore, a shift can be observed by increasing the target concentration (Figure 2-4 A). I incubated 200nM latest round library with different concentration of LH and FSH and ran the polyacrylamide gel to get a rough idea about the binding affinity and specificity of the latest selected library. As can be seen in the Figure 2-4 B, there is an increasing shift trend from low LH concentration $(0.85 \mu \mathrm{M})$ to high $\mathrm{LH}$ concentration $(6.8 \mu \mathrm{M})$, whereas no shift can be observed for all FSH concentrations, same as the negative control (ssDNA library without any protein incubation). One difference between ELONA and EMSA designed is that, in the ELONA, protein concentration is fixed and ssDNA libraries concentration is changed. Whereas, in the EMSA, I set the ssDNA libraries concentration and change protein concentration. By using these two methods, the binding affinity of the lasted round library was confirmed to be the strongest and no FSH interaction in both studies. The result gave a strong confidence to stop the selection and carry on further individual sequence characterisation.

A

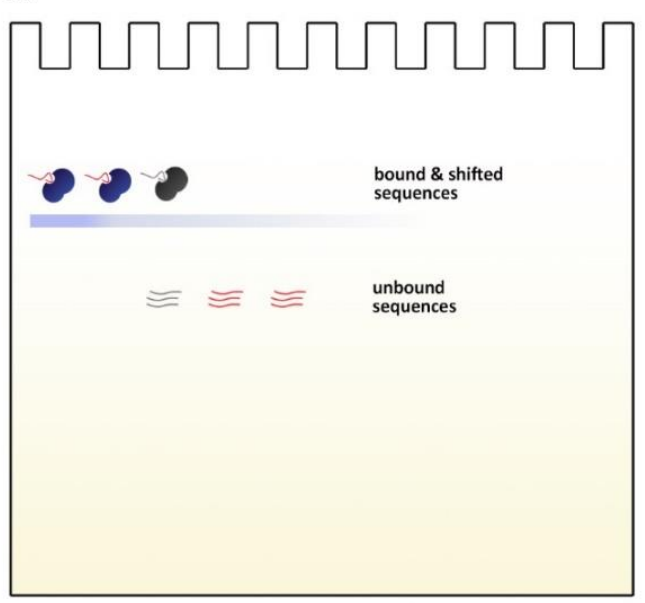

B
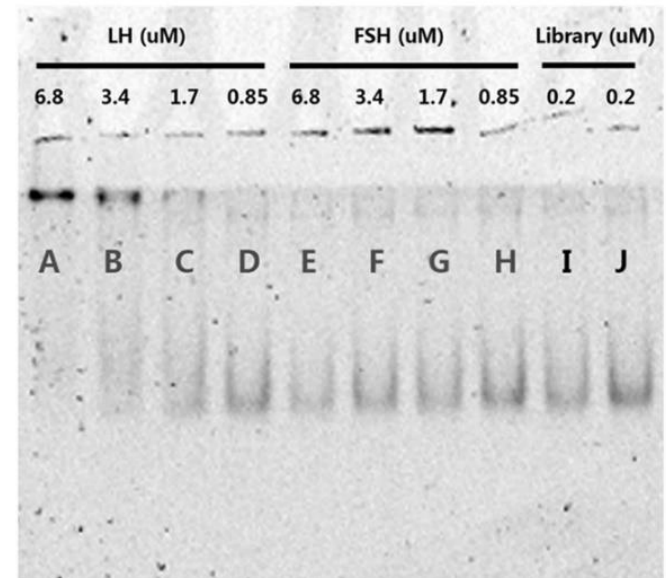
Figure 2-4: EMSA study to further confirm the binding affinity and specificity (A) illustration of EMSA mechanism. The more sequence bound to the protein, the stronger intensity of the band appeared (Blue line). (B) EMSA gel electrophoresis image for the $20^{\text {th }}$ library against $\mathrm{LH}$ and $\mathrm{FSH}$, from D to A lanes is the titration of $\mathrm{LH}$ against $20^{\text {th }}$ round library. From $\mathrm{H}$ to $\mathrm{E}$ lanes are the titration of FSH against $20^{\text {th }}$ round library. The negative control was lane I and J, both of them were library incubated with buffer alone.

\subsection{Sequence analysis}

\subsubsection{Cloning and Sanger sequencing}

After the LH binding affinity and specificity of the latest selection round library were confirmed, the library pool was cloned and sent for Sanger Sequencing. In total 40 clones were sent but only 38 sequences were obtained (two clones have mismatch primer regions). The sequence alignment and population can be seen in Figure 2-5 A, among all the 38 sequences, B10 appeared eight times, and B38 appeared four times. B11, B35 and B10 shared a very similar sequence order, which they only have one nucleotide difference. This might suggest B11 and B35 are the mutation forms of $\mathrm{B} 10$ since the population of these two sequences is less than B38. Therefore, I decided to use B10, B38, B22, B18, B23 and B21 as the final aptamer candidates. I observed most of these sequences are "G rich" sequence. Therefore there is quite a high chance for them to form $\mathrm{G}$ quadruplex structures. To further investigate their possible structure, I used MFold to predict the secondary structure according to the free energy [115]. Figure 2-5 B shows the secondary structures of these six selected aptamer candidates predicted by Mfold software. Form the predicted model, most of the candidates formed loops together with the primer regions. However, further investigation needs to be done to investigate the whether the primer regions also involved in the $3 \mathrm{D}$ structure the sequences form for the binding of LH. 
A

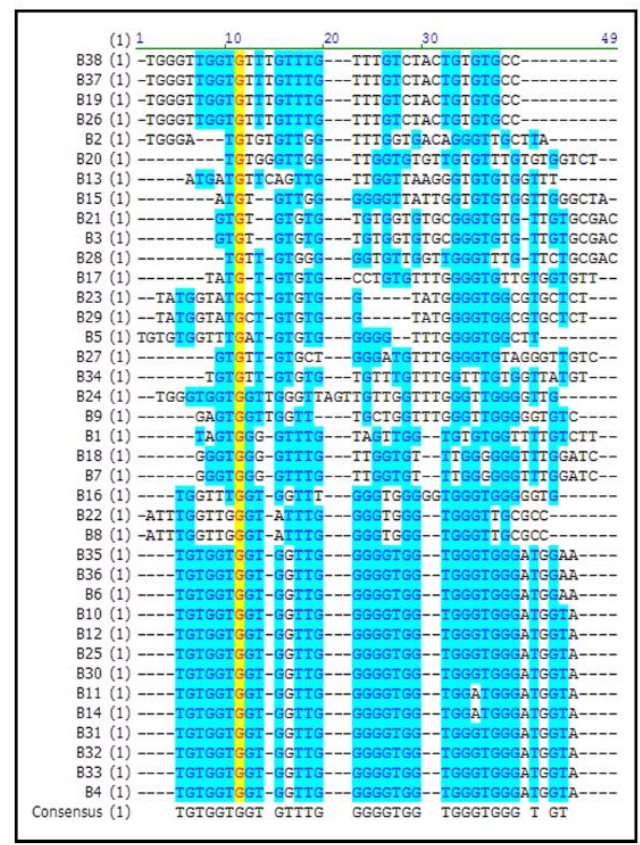

\begin{tabular}{|l|l|l|}
\hline Sequence & Frequency & Name \\
\hline TGTGGTGGTGGTTGGGGGTGGTGGGTGGGATGGTA & 8 & B 10 \\
\hline TGGGTGGTGTTTGTTGTTTGTCTACTGTGGCC & 4 & B 38 \\
\hline TGTGGTGGTGGTTGGGGGTGGGGATGGGATGGTA & 2 & B 11 \\
\hline TGTGGTGGTGGTGGGGGTGGTGGGTGGGATGGAA & 2 & B 35 \\
\hline ATTTGGTTGGGTATTGGGGTGGGTGGGTGCGCC & 2 & B22 \\
\hline GGGTGGGGTTGTTGGTGTTTGGGGGGTTGGATC & 2 & B18 \\
\hline TATGGTATGCTGTGTGGTATGGGGTGGCGTGCTCT & 2 & B 23 \\
\hline ATGTGTTGGGGGGTTATTGGTGTGTGGTTGGGCTA & 2 & B21 \\
\hline \hline
\end{tabular}

\section{B}

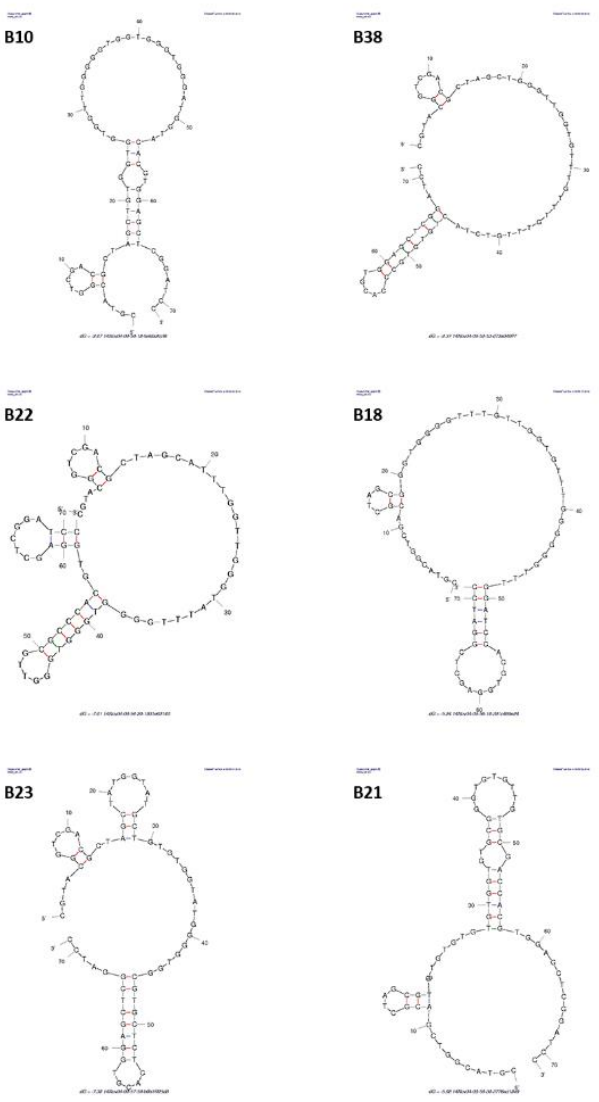

Figure 2-5: Sequence analysis. (A) Global alignment of all the sequence obtained from Sanger Sequencing, most of the sequences are G rich. The table below summarised the appearance frequency of all the sequence that can be found more than two copies of the received sequencing data. B10, B11 and B35 share very similar sequence. (B) MFold prediction of the major sequences, these six sequences were picked as they appear the most among the whole library pool.

\subsection{Affinity and specificity characterization for picked candidates}

\subsubsection{Screening of best candidate by ELONA}

$\mathrm{B} 10, \mathrm{~B} 38, \mathrm{~B} 22, \mathrm{~B} 18, \mathrm{~B} 21$, and B23 from the latest selection round were picked as the aptamer candidates for further characterisation of the affinity and specificity against LH. I first use ELONA to screen out the best binder among all the sequences. All of the six sequences were custom-synthesised with a biotin label at the 5'end site so that after they bind to the LH immobilised on the plate, Streptavidin-HRP and TMB can be added for a colorimetric signal generation. In the initial screening, 
200ng of LH was immobilised on each well and concentration of $0,10,100500$, 1000,2000 and 5000nM of each aptamer candidates were added for titration. Figure 2-6 shows the binding curve for all the six aptamer candidates and their Hyperbl fitting. Among the candidates, B23, B10 and B38 shows relatively strong $K_{D}$ and $B 23$ is one of the best binder $\left(K_{D}=334 n M\right)$. B23 was decided to choose as the aptamer candidate for further affinity characterisation.

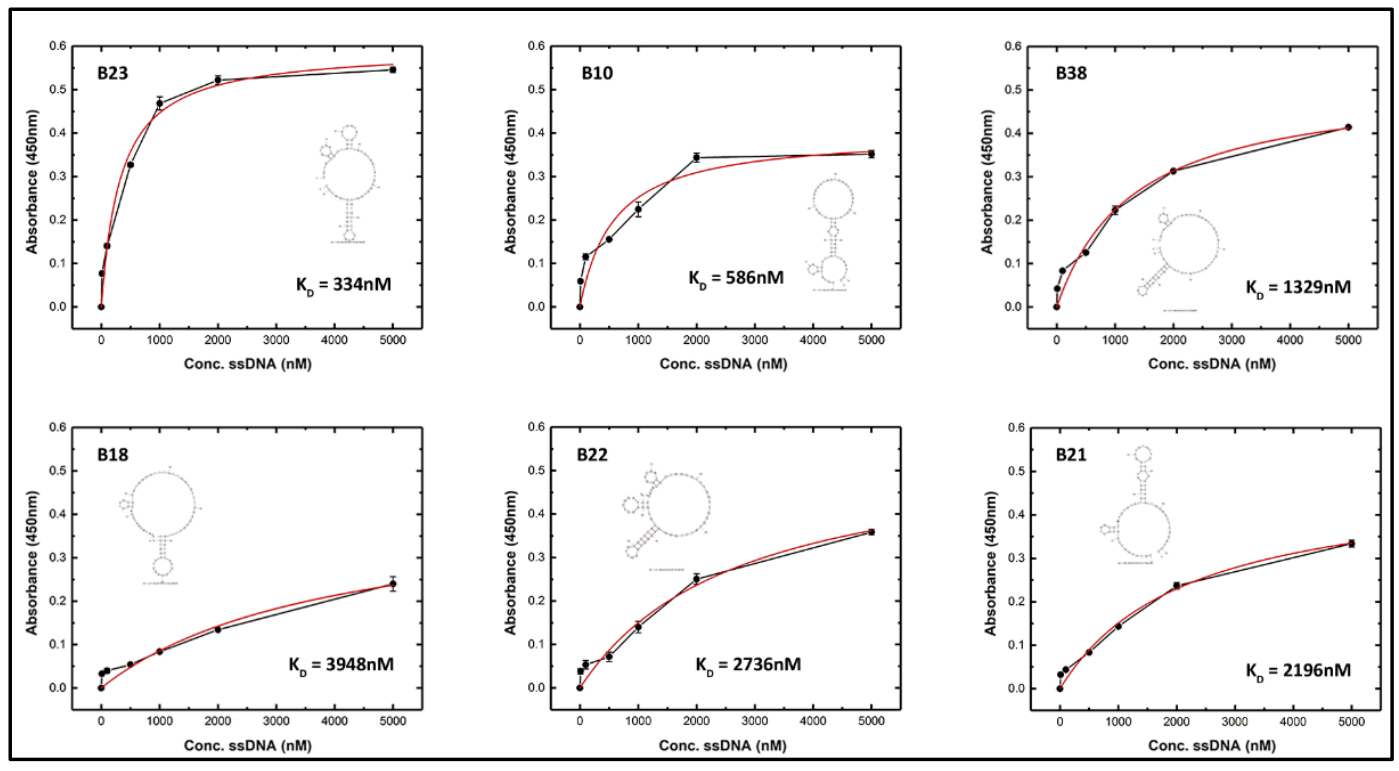

Figure 2-6: Binding affinity selected candidates. ELONA studies on all the 6 aptamer candidates, 7 concentration points were used from $0 \mathrm{nM}$ to $5000 \mathrm{nM} . \mathrm{K}_{\mathrm{D}}$ of each aptamer candidates were obtained via fitting the data points to a Hyperbl model.

\subsubsection{Truncation study of selected aptamer}

Sometimes the primer regions from the library would involve in forming the binding region of the aptamer against the target. Therefore I want to investigate further whether truncation of this two-primer region will affect B23's binding affinity. A full length and truncated B23 were synthesised with one end of biotin label for ELONA. Figure 2-7 A shows the result of this ELONA study. This time a similar setup was used like the previous screening test together with two more negative controls. One was testing the response for truncated B23 sequences against $\mathrm{FSH}$, and the other was testing the signal response of a random sequence obtained from another group member against LH. As can be seen in the result, there was no apparent assay signal for both negative controls (Figure 2-7 A, navy blue and light blue) whereas noticeable dose-response curve can be observed for the two B23 
sequences (Figure 2-7 A, blank and grey). After fitting of the data to a Hyperbl model, the $K_{D}$ calculated was $321 \mathrm{nM}$ for the full-length sequence and $451 \mathrm{nM}$ for the truncated sequence. The $\mathrm{K}_{\mathrm{D}}$ slightly decreased but I decided to use the short B23 sequence for further characterisation as the shorter B23 sequence is more straightforward for further sensor design and it remains a relatively strong binding affinity and specificity against LH. The B23 in the rest of this thesis represents the truncated sequence (35-nt).

\subsubsection{Specificity study of selected aptamer}

I tested the cross-reaction of a range of proteins that may affect sensor application to B23. There are two major groups: one is gonadotropins that would appear in infertility patient's blood (FSH and TSH). The other is abundant proteins that exist in normal human blood (HSA and IgG). I used both ELONA and EMSA for the investigation. All of these proteins (500ng each) were immobilised on the plate, $5 \mu \mathrm{M}$ of B23 was added to test the signal response of FSH, TSH, HSA, and IgG. $1 \mu \mathrm{M}$ of B23 was added to test the signal response of $\mathrm{LH}$. As can be seen in the Figure 2-7 B, only LH shows a significant signal response. I also carried out EMSA to double confirm the possible cross-reaction for B23 against HSA and FSH - these two are more critical in the clinical application. In this study, I applied $5 \mu \mathrm{M}$ of FSH and HSA and a serial concentration (from 0 to $5 \mu \mathrm{M}$ ) of LH against B23. As a result shown in Figure 2-7 C, no significant shift can be observed for both FSH and HSA lane. However, there is an increasing shift for the LH lanes which indicated the binding effect. Another negative control of random sequence also been used in this experiment and no shift can be observed.

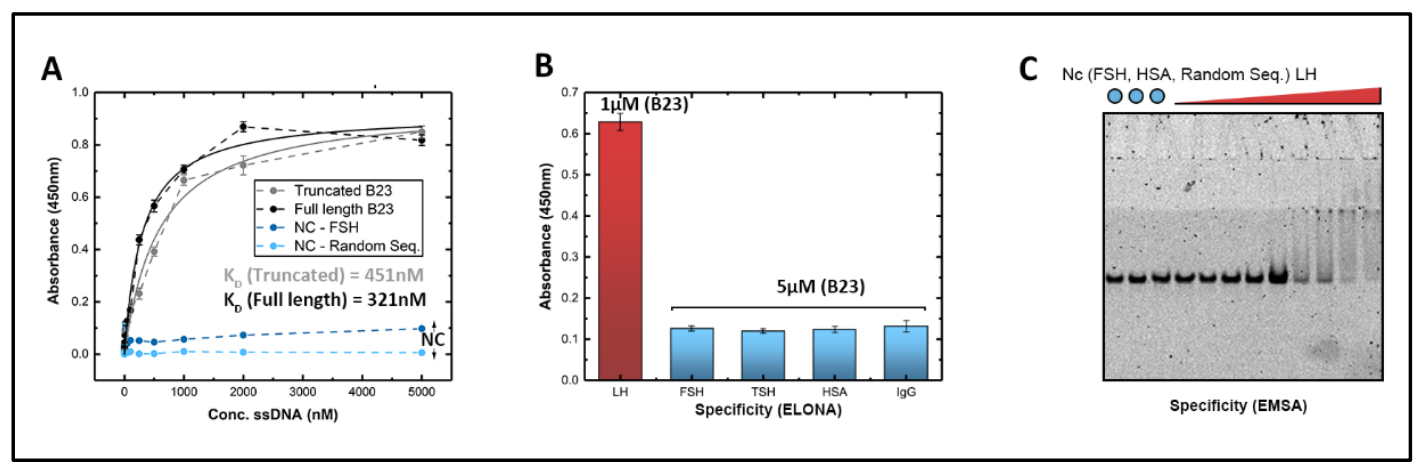

Figure 2-7: Binding affinity and specificity of selected candidates. (A) Truncation study of B23. The dose response curve of truncated B23 is shown in grey colour and the full length is shown in black. Two blue lines indicate the negative controls, one is the response of truncated B23 against FSH (blue), the other is the response of a random sequence against 
LH (light blue). (B) Specificity study of B23 via ELONA. The red column represents the colorimetric response of adding $1 \mu \mathrm{M}$ B23 onto the $\mathrm{LH}$ plate and the blue columns represent the response of adding $5 \mu \mathrm{M}$ of B23 to other non-specific proteins. (C) Specificity study of B23 via EMSA. The blue dots represent the negative controls of the experiment which are running $1 \mu \mathrm{M}$ of B23 with $5 \mu \mathrm{M} \mathrm{FSH}$, running $1 \mu \mathrm{M}$ of B23 with $5 \mu \mathrm{M}$ HSA and a random sequence with $5 \mu \mathrm{M} \mathrm{LH}$. The rest of the lanes are running $1 \mu \mathrm{M}$ of $\mathrm{B} 23$ with a series of $\mathrm{LH}$ concentration ranging from 0 to $5000 \mathrm{nM}$.

\subsection{Binding kinetics and conformational changing dynamics studies}

\subsubsection{Surface Plasmon Resonance (SPR) study for selected aptamer}

As a well-developed biophysical technique, SPR has been widely used for binding interaction study. It applies an optical method based on detection of the subtle change of the refractive index of the medium near a metal surface such as gold. One major advantage of SPR compare to other affinity techniques is it can also investigate the binding kinetics of the binding molecule against the target [116].

In the SPR study design, B23 sequence was synthesised with a thiol label at one end for the immobilisation on the bare gold SPR chip. After the immobilisation of B23 on to the chip and back-filling co-thiol molecules $\mathrm{MCH}$ (a more detailed discussion on the usage of co-thiol molecules can be found in the section 3.3 of Chapter 3), different concentration of LH was injected with a certain flow rate to allow contact of the target and the B23 aptamer. In between each LH injection, a $7 \mathrm{M}$ Urea was injected for the regeneration of the sensor surface. Figure 2-8 A shows the injection-association-dissociation-regeneration cycle from 50nM LH injection to $500 \mathrm{nM} \mathrm{LH}$ injection. It's quite clear to see the $7 \mathrm{M}$ Urea can fully regenerate the sensor (SPR signal back to the baseline). 6 concentrations from $10 \mathrm{nM}$ to $10 \mu \mathrm{M}$ LH were applied (buffer as blank) and the SPR responses were fitted to a 1:1 binding model (Figure 2-8 B), the estimated $\mathrm{K}_{\text {off }}=1.2 \times 10^{-3} \mathrm{~s}^{-1}$. It's a relatively fast off rate (slow off-rate normally $<10^{-5} \mathrm{~s}^{-1}$ ), which this feature may benefit to continues sensing format design. I also calculated the $\mathrm{K}_{\mathrm{D}}$ using this fitted model and it was estimated as $535 \mathrm{nM}$. The result remains quite consistent with few of our other affinity assay methods with the acceptable scale of variation. 

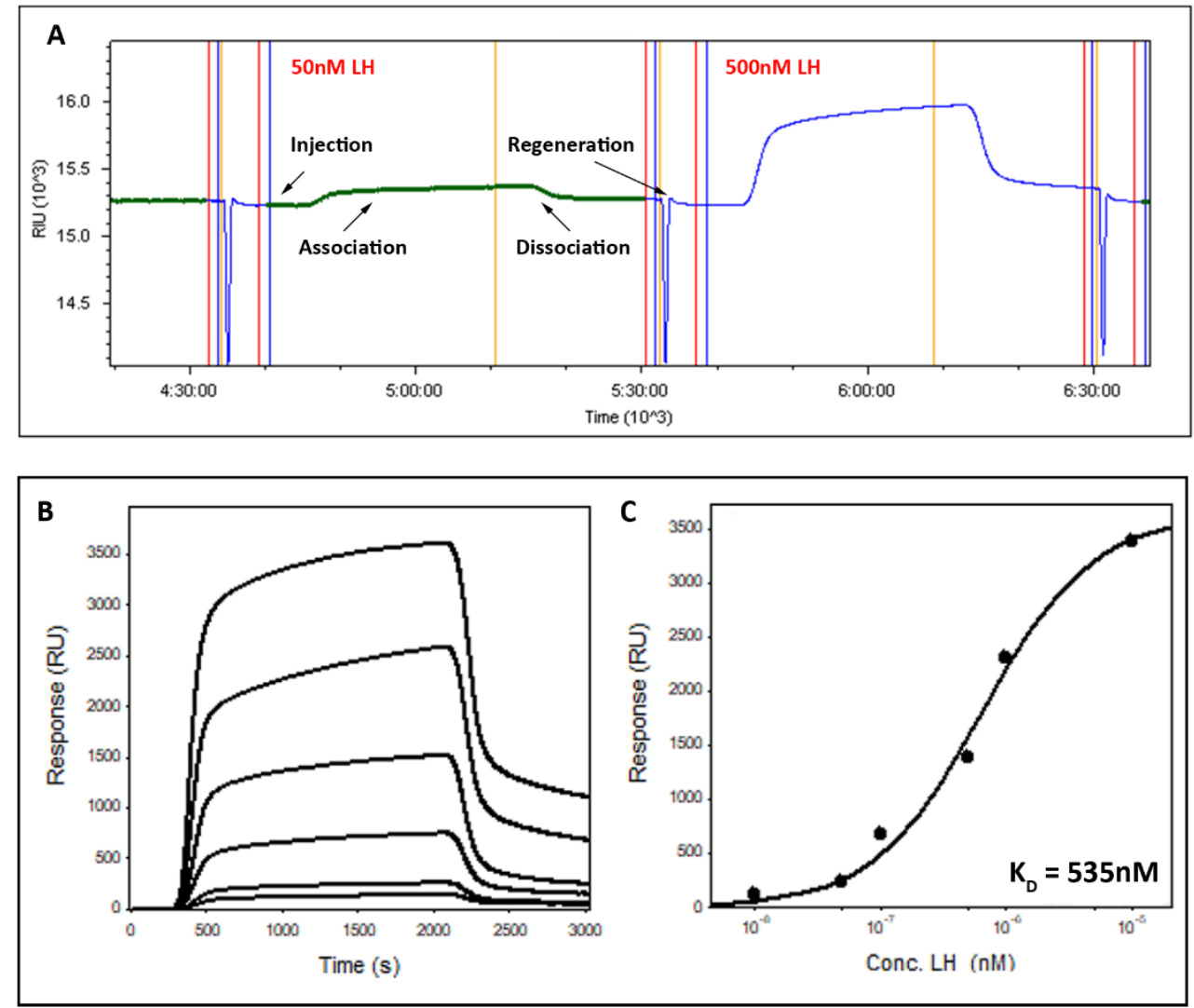

Figure 2-8: SPR study to investigate the binding kinetics of B23. (A) Injectionassociation-dissociation-regeneration cycles of the SPR study. Different stages are pointed out by the black arrows and the figure includes two concentrations, one is the response of the B23 aptamer against 50nM LH and the other is response of 500nM LH. (B) SPR response of the B23 aptamer against different concentrations of $\mathrm{LH}$ from $10 \mathrm{nM}$ to $10000 \mathrm{nM}$. (C) The affinity estimation from the scrubber programme. $\mathrm{K}_{\mathrm{D}}$ is estimated to be $535 \mathrm{nM}$.

\subsubsection{Circular Dichroism (CD) study for selected aptamer}

From the sequencing data, I noticed the latest round library may contain a certain portion of G-quadruplex structure aptamers. To further investigate their possible secondary structure, $\mathrm{CD}$ experiment was performed to study whether the 6 candidates form any G-quadruplex structures. G-quadruplex structures arise from different G-quartet stacking, strand segment orientation and loop arrangements. These particular features lead to the display of unique CD spectral signatures. For example, a parallel G-quadruplex structure shows a positive peak at $264 \mathrm{~nm}$ and negative peak at $245 \mathrm{~nm}$, whereas an antiparallel structure often shows a positive 
peak at $295 \mathrm{~nm}$ and negative peak at 260nm [117].

Figure 2-9 A shows the $\mathrm{CD}$ experiment result for B21, B18, B38, B21, B23 and B10. It's clearly to see B18 and B10 formed parallel G-quadruplex structure as there were strong absorption for a positive peak in $265 \mathrm{~nm}$ and a negative peak in 240nm. B23 also forms another type of G-quadruplex structure different from B18 and B10 with a positive peak at $280 \mathrm{~nm}$ and negative peak at $240 \mathrm{~nm}$. A further CD study of B23 was then performed to investigate whether the binding of LH will change the confirmation. Figure 2-8 B shows the CD result before and after adding $1 \mu \mathrm{M} \mathrm{LH}$, the negative peak at $240 \mathrm{~nm}$ became more negative and the positive peak at $280 \mathrm{~nm}$ increased. It is believed that there is conformational change for B23 upon binding to LH. This feature is important as in the electrochemical biosensor design, conformational change is needed to form a good sensor.

A

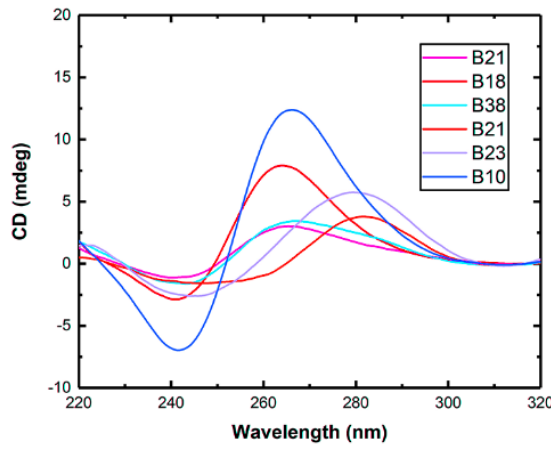

B

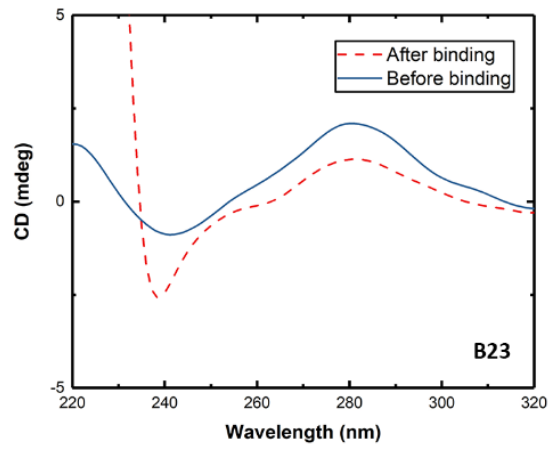

Figure 2-9: Conformational study on aptamer candidates (A) CD of 6 aptamer candidates. B10 and B18 shares similar G-quadruplex structures and B23 shows another kind of G-quadruplex structure. No obvious G-quadruplex structures can be depicted from other candidates (B) CD of B23 before and after binding $5 \mu \mathrm{M}$ of LH. The red dash line represents the change after binding, negative peak at $240 \mathrm{~nm}$ increased and a positive peak at $280 \mathrm{~nm}$ also increased.

\subsection{Chapter summary}

The generation of a new binding molecule against LH is critical for the development of new sensing method for LH pulsatility. In this chapter, I developed the binding molecule by a nitrocellulose membrane-based SELEX method (Figure 2-2). 6 candidates were obtained (Figure 2-5 B) and I selected B23 as the best binder via comparing their $\mathrm{K}_{\mathrm{D}}$ estimated by ELONA experiment (Figure 2-6). To consolidate the binding affinity data, I used both biochemical methods (ELONA and EMSA) 
(Figure 2-7) and biophysical method (SPR) (Figure 2-8 C) to estimate $\mathrm{K}_{\mathrm{D}}$ value of the truncated B23 against LH. All the values obtained were in consistent with a variant about from $334 \mathrm{nM}-535 \mathrm{nM}$. I consider the variation is acceptable as different methods may result in different value amount. The specificity of B23 also been investigated by EMSA and ELONA, I have not observed any cross-reactions among FSH, TSH, HSA, and IgG (Figure 2-7). SPR was used to further investigate the LH binding kinetic of B23. After fitting to a 1:1 binding model, the $\mathrm{K}_{\text {off }}$ was estimated to be $1.2 \times 10^{-3} \mathrm{~s}^{-1}$. It's a relatively fast off rate (slow off-rate normally $<10^{-5} \mathrm{~s}^{-1}$ ) (Figure 2-8). However it is still one level below when compared to some of other small molecules aptamer (normally $=10^{-2} \mathrm{~s}^{-1}$ ). Therefore, how I can better regenerate the binding capacity is critical for the electrochemical sensing platform development. However, the feature of confirmed structural change gave us the confidence to use B23 as the final candidate of the LH sensor. Clear conformational change was observed from the $\mathrm{CD}$ experiment before and after adding $\mathrm{LH}$ onto a B23 solution (Figure 2-9). In conclusion, after the SELEX, I obtained an LH specific aptamer candidate which suitable to applied electrochemical format for further sensor development.

\subsection{Materials and Methods}

\subsubsection{Materials}

All chemicals and reagents were of analytical laboratory grade or higher. Proteins used for selection were the recombinant LH (Luveris ${ }^{\circledR}$ ) and recombinant FSH (Gonal-f®), both were purchased from Merck Serono. Human pituitary LH, FSH, TSH were purchased from Lee BioSolutions, they were used for aptamer characterisation. Other proteins for characterisation including HSA, BSA and IgG were purchased from Sigma Aldrich. All of the oligonucleotides including DNA library, modified primers, and aptamer candidates from this chapter were custom synthesised from Integrated DNA Technologies (IDT). Nitrocellulose membrane and its filter accessories were purchased from Millipore. Dnynabeads ${ }^{\circledR}$ MyOne ${ }^{\mathrm{TM}}$ streptavidin T1 was purchased from ThermoFisher Scientific. Pfx PCR kit was purchased from Invitrogen. $\lambda$-exonuclease kit was purchased from New England Biolabs. 


\subsubsection{Nitrocellulose membrane filtration}

The SELEX procedure used in this chapter was based on a nitrocellulose membrane filtration method which utilise a $0.45 \mu \mathrm{m}$ HAWP nitrocellulose membrane and its filter accessories (illustrated in Figure 2-1 B). For example, in the initial round of selection, 10nmole of ssDNA library and 100pmole of the recombinant LH were used. The ssDNA library was first heated to $96{ }^{\circ} \mathrm{C}$ and cooled down at room temperature before every selection round. This library was incubated with the recombinant $\mathrm{LH}$ on a rotating platform at room temperature for 1 hour. The mixture was filtered through the nitrocellulose membrane and washed three times to remove the non-specifically bound ssDNA. This membrane was then heated at $99{ }^{\circ} \mathrm{C}$ for 5 minutes in $300 \mu \mathrm{L}$ to denature protein and elute the bound ssDNA. PhenolChloroform extraction and ethanol precipitation were used to recover the bound ssDNA before PCR amplification. The reset of selection rounds and counter selection rounds were performed under similar protocol with different library: protein ratio as mentioned in the result sections.

\subsubsection{PCR amplification and agarose gel electrophoresis}

The PCR amplification was performed using PCR mix listed in Table 2-1 under parameters: initialization step $\left(3\right.$ minutes, $\left.95{ }^{\circ} \mathrm{C}\right)$, denaturation step $(30$ seconds, $95{ }^{\circ} \mathrm{C}$ ), annealing step $\left(30\right.$ seconds, $\left.51{ }^{\circ} \mathrm{C}\right)$ and extension step at $\left(30\right.$ seconds, $\left.72{ }^{\circ} \mathrm{C}\right)$. The PCR product collected from each round was sacrificed $1 \mu \mathrm{L}$ to be separated by $3 \%$ agarose gel electrophoresis in 1X TAE (Tris-acetate-EDTA) buffer at $400 \mathrm{~V}$. The gel was stained with SYBR gold and photographed under the UV light. and stored at $-20{ }^{\circ} \mathrm{C}$ freezer until the next experiment.

Table 2-1 Pfx PCR reaction mixture

\begin{tabular}{ll}
\hline Reagent & Volume $(\mu \mathrm{L})$ \\
\hline $10 \mathrm{x}$ Buffer & 10 \\
$\mathrm{ddH}_{2} \mathrm{O}$ & 39.6 \\
$\mathrm{FP} 10 \mu \mathrm{M}$ & 20 \\
$\mathrm{RP} 10 \mu \mathrm{M}$ & 20 \\
$\mathrm{dNTP}$ mix $10 \mathrm{Mm}$ & 10 \\
Pfx DNA polymerase & 0.4 \\
\hline Total volume & 100 \\
\hline
\end{tabular}




\subsubsection{Generation of ssDNA by Biotin-streptavidin separation}

The double-stranded DNA PCR product was applied to streptavidin magnetic beads for the separation of non-biotinylated single-stranded DNAs from their complementary biotinylated strand. The beads were pre-washed three times by the $1 \mathrm{X}$ binding \& wash $(\mathrm{B} \& \mathrm{~W})$ buffer recommended in the product protocol. After the pre-washed step, $95 \mu \mathrm{L}$ PCR product and $2 \mu \mathrm{L} 2 \mathrm{X}$ B\&W buffer was then added to the beads and mixed for 30 minutes at room temperature on a rotating plat-form. The mixture was then applied to a magnet and the supernatant was removed. The selected single-stranded DNA sequences were separated from their complementary strands (with a biotin modified 5' end) by incubating with $50 \mu \mathrm{L} 0.1 \mathrm{M} \mathrm{NaOH}$ and elute after 5 minutes. $150 \mu \mathrm{L}$ binding buffer was then added and concentration was measured by a Nanodrop-2000 (Thermo Fisher Scientific).

\subsubsection{Enzyme Linked OligoNucleotide Assay (ELONA)}

Enzyme Linked OligoNucleotide Assay (ELONA) was performed with 4 different selection rounds (Round 3, Round 7, Round 15 and Round 20). 160 ng of LH and FSH in $100 \mu \mathrm{L}$ selection buffer were coated on each well of the 96-well microtitre plates overnight at $4^{\circ} \mathrm{C}$ separately. After blocking with PBS Tween-20 (PBST) buffer with $1 \%$ BSA for 1 hour at room temperature, the wells were washed 3 times with the selection buffer. A biotinylated complementary strand for the 5 'end primer region was annealed to Round 3, Round 7, Round 15 and Round 20. These biotinylated pools in different concentrations $(1 \mu \mathrm{M}, 500 \mathrm{nM}$, and $50 \mathrm{nM})$ were added to the LH-coated wells/FSH-coated wells and incubated for 1 hour at room temperature. Following 3 times washes, streptavidin-horseradish peroxidase (HRP) conjugate was applied to each well. The plate was incubated for 30 minutes and washed with $0.1 \%$ BSA PBST buffer. $50 \mu \mathrm{L}$ TMB was then added to the wells and incubated for 30 minutes. By adding $100 \mu \mathrm{L}$ of $1 \mathrm{M} \mathrm{H} 2 \mathrm{SO} 4$, the reaction was quenched and the binding complexes were quantified by measuring the absorbance at $450 \mathrm{~nm}$ in the micro plate reader. The further characterisation of the 6 aptamer candidate were performed using similar ELONA setup but with different range of aptamer concentration for the titration. 


\subsubsection{Electrophoretic Mobility Shift Assay (EMSA)}

The latest selection pool Round 20 was tested for its binding affinity to LH using Electrophoretic Mobility Shift Assay (EMSA). The sample pool was prepared by magnetic bead-based ssDNA generation after the amplification of the Round 20 library as described previously. $0.2 \mu \mathrm{M}$ Round 20 ssDNA pool was then incubated with increasing concentrations of LH and FSH from $0.85-6.8 \mu \mathrm{M}$ in a total volume of $10 \mu \mathrm{L}$ at room temperature. After 1 hour incubation, the samples were resolved by double layers non-denaturing polyacrylamide gels $(6 \%$ polyacrylamide for stacking and $12 \%$ polyacrylamide for resolving) containing $1 \mathrm{X}$ TBE buffer. The gel was stained with SybrGold for 30 minutes and imaged on the GelDocTM XR+ system (Bio-rad). The further characterisation of the B23 aptamer candidate was performed using similar EMSA setup but with a different range of aptamer concentration for the titration.

\subsubsection{Cloning and sequencing}

Round 20 were chosen for cloning and sequencing analysis. The Round 20 was amplified with unmodified primers and purified using the PCR clean-up system (Qiagen). The product was cloned into a PCR Blunt II TOPO vector (Invitrogen) and transformed into E.Coli DH alpha competent cells via heat shock method. 40 colonies of each pool were picked and purified using the mini-prep kit (Qiagen). 40 samples in total were prepared and sent to sequence (Techdragon). All the sequences data collected were aligned using Vector NTI Advanced 11.1 (Invitrogen) and predicted the secondary structure by MFold with a setting of the condition according to the selection buffer.

\subsubsection{Surface Plasmon Resonance (SPR)}

As the same for the electrochemical system, the probe used for the SPR study modified with thiol at one end. $1 \mu \mathrm{M}$ of B23 was immobilized onto a plain gold chip in an overnight incubation at room temperature. After the immobilization, $1 \mathrm{~mL}$ of $2 \mathrm{mM}$ MSA was used to blocking the unoccupied binding sites on the gold surface. The plain gold chip was then adapted into a flow cell of the Reicherts SPR platform. SPR optimization 8 The SPR measurement was fine-tuned by adjusting the time 
settings and flow rates of injection, dissociation, waiting and regeneration. The final setting was as bellowed: 40 mins of injection, 40 mins of dissociation following another 40mins injection of the regeneration solution, 40 mins of dissociation and 40 mins of waiting in $2 \mu \mathrm{L} / \mathrm{min}$ flowrate. Regeneration Different regeneration solutions were tested in order to obtain a better regeneration efficiency: $10 \mathrm{mM}$ Glycine in $\mathrm{pH} 2,10 \mathrm{mM}$ Glycine in $\mathrm{pH} 10$ and $50 \mathrm{mM}$ of $\mathrm{NaOH}$. Same concentration of the analyte was injected repeatedly 6 times in these 3 regeneration solution and the relevant respond units were analysed.

\subsubsection{Kinetic calculation}

The affinity interactions between immobilised aptamer and LH in the solution were characterized by the association rate constant $\left(k_{\mathrm{on}}\right)$, dissociation rate constant $\left(k_{\mathrm{off}}\right)$, and equilibrium association constant $\left(K_{\mathrm{a}}\right)$. The sensitivity of the $k_{\text {on }}$ and $k_{\text {off }}$ in response to variation of the binding conditions are the main study points based on the concept of "kinetic discrimination" [118]. The data were fitted using a simple 1:1 reversible interaction model, $A+B \leftrightarrow A B$, where $A$ is the injected LH, $B$ is the immobilised aptamer, and $A B$ was the the-aptamer complex formed during the reaction. In the SPR system, the signal $R$ was proportional to the amount of $[A B]$, and $R_{\max }$ was proportional to the initial $[B]$. A set of differential equations was applied to the data to find a solution for the three parameters $k_{\mathrm{on}}, k_{\mathrm{off}}$, and $K_{\mathrm{a}}$, respectively from the Analysis interface Scrubber

\subsubsection{Circular Dichroism (CD)}

$5 \mu \mathrm{M}$ of 6 aptamer candidates were measured in the CD (Jasco J-810 spectropolarimeter). Smooth processing was done using the software provided by the manufacturer. In the conformational change study, $5 \mu \mathrm{M}$ of B23 was used (volume $=300 \mu \mathrm{L}$ ), and $5 \mu \mathrm{L}$ of $30 \mu \mathrm{M} \mathrm{LH}$ was added to $295 \mu \mathrm{L}$ of $5 \mu \mathrm{M}$ B23. The $\mathrm{CD}$ was scanned from 220 to $320 \mathrm{~nm}$. The data gathered were the average of four scans at a scanning rate of $100 \mathrm{~nm} / \mathrm{min}$. The scan of the buffer recorded at room temperature was subtracted from the average scans for each DNA sequence. Data were processed in the Excel and Origin for plotting. 


\section{CHAPTER 3 | Electrochemical aptamer-based LH assay development}

In this chapter, I focus on developing the electrochemical assay which applies our selected aptamer as the sensing element to quantify LH level. The method converts the binding event between LH and the redox probe labelled LH aptamer into a clear electric signal driven by binding-induced electron transfer kinetics of the probe. I present the optimisation of assay parameters such as redox probe selection, aptamer packing density, and backfilling co-thiol molecules optimisation under a standard disk electrode system. I also present the validation of the assay performance via different electrochemical techniques such as $\mathrm{CV}, \mathrm{SWV}$, and ACV to investigate the optimal signal analysing technology which will be applicable in the final device setup.

Keywords: E-AB, Square-wave voltammetry, Alternating current voltammetry

\subsection{Background}

Electrochemical aptamer sensors provide many advantages over other detection techniques regarding sensitivity and specificity. The commercial availability of chemically modifying any aptamer sequence with electrochemical label offers a convenient to adapt aptamer to conformational change-based electrochemical assay format without further design [119]. However, the critical requirement of this assay format is the need and an optimal degree of conformational change, which limits the generalisation of this type of assay since not all the aptamer-target binding will trigger aptamer conformational change. White et al. reviewed the advantage and challenge of using this sensing format for diagnostic device development [120]. In recent years, the positive process has been made to improve $\mathrm{E}-\mathrm{AB}$ performance by optimising the electrode fabrication method [121-123] and choosing appropriate electrochemical techniques [109]. Application of using E-AB in real-time monitoring of drug pharmacokinetics in the awake animal has also been achieved in Plaxco's group [124]. 
There are five main electrochemical techniques utilised in the literature include cyclic voltammetry(CV), differential pulse voltammetry (DPV), square wave voltammetry (SWV), alternating current voltammetry (ACV) electrochemical impendence spectroscopy (EIS). In our study, I used CV, SWV and ACV for the characterisation of our aptamer sensing system.

Cyclic Voltammetry (CV) involves measuring current over a potential range and switching the direction of the scan at a specific potential, then the current is measured in the opposite direction of the original scan before the scan direction again changes at the other end of the potential range and proceeds in the original direction. This 'sweeping' as it is often referred to may be repeated several times as changes in the system are observed. This technique is often used when a redox couple is present in the system, as changes in the positioning or accessibility of the redox probe to the working electrode are detectable as peak current changes. Square wave voltammetry (SWV) is commonly seen as one of the most sensitive techniques. It involves the application of a potential ramp and applying a small pulse of constant amplitude to it. SWV can also be seen as using asymmetrical double pulse, one in the direction of the scan (forward) direction and one in reverse, this results in a square wave been superimposed on a potential staircase. Each current measurement is taken at the end of the individual pulses. A difference current is plotted against potential. This technique provides the excellent background noise suppression of DPV, as charging currents are allowed to decay, while an increased sensitivity is noted due to the recording of both forward and backwards currents. Finally, the technique allows for vastly improved speeds over other techniques as the increased samples taken provides for increased frequency while maintaining its sensitivity. An SWV scan can occur in a matter of seconds compared to minutes for other techniques.

In this chapter, I aim at

1) Using the electrochemical techniques above to identify the optimal assay parameters for the $\mathrm{E}-\mathrm{AB}$.

2) Characterising the sensor performance by different electrochemical techniques. With all of these established on a well-verified traditional gold disk electrode system, I can move forward more customised sensing platform design. 


\subsection{Redox probe comparison}

Redox-reporter-labelled oligonucleotides have been widely used in electrochemical biosensor designs. In E-AB sensors, the most commonly used redox probes are Methylene blue (MB), Ferrocene $(\mathrm{Fc})$. Other redox reporters such as Nile blue and anthraquinone have also been investigated by varies research groups (Figure 3-1 A). These probes are commercially available to conjugate onto the aptamer and active at potential within the $1 \mathrm{~V}$ window over which thiol-on-gold SAMs are reasonably stable. In this study, I compared the use of $\mathrm{MB}$ and $\mathrm{Fc}$ as the redox label on LH aptamer and found out MB has better performance whereas the Fc labelled aptamer showed less signal stability and may not suitable for application in some of the commercially available chip-based electrodes due to peak potential overlaps from our results.

\subsubsection{Probe modification}

Ferrocene and Methylene blue modified aptamer can be purchased from companies provide oligonucleotide synthesis services such as IDT (USA), Biomers (German) and Sangon (China). Methylene blue modification provided by those companies usually achieved by post synthesis conjugation - coupling 3'-amine-terminated oligos with NHS ester-activated while Fc modification can be directly linked to the base nucleotide or coupled using similar post-synthesis modification (Figure 3-1 B). In the studies, I ordered all the MB modified sequences from Biomers and modified Ferrocene labelled aptamer in-house. The in-house modification was achieved via coupling Ferrocene carboxylic acid onto the B23 sequence with a 5' amino modifier. A detailed protocol is described in the method section.
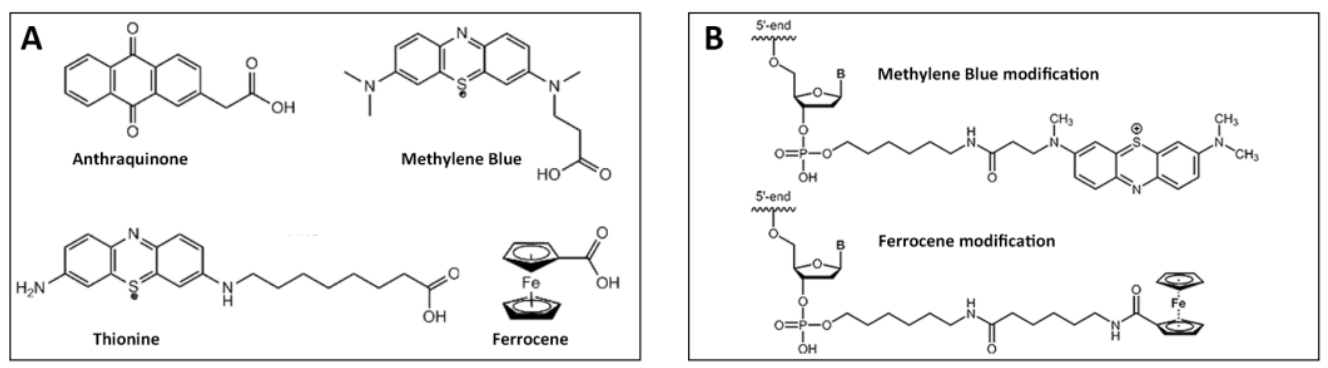

Figure 3-1: Commercially available redox modifiers. (A) Anthraquinone, Methylene Blue, Thionine and Ferrocene are four of the most frequently use electrochemical redox probe. (B) The oligo modifications using Methylene Blue and Ferrocene. 


\subsubsection{The issue of using $F c$ as a label}

Both $\mathrm{MB}$ and $\mathrm{Fc}$ are ideal candidates to use as signalling moieties because of their reversible electrochemical behaviour and commercially available conjugation chemistry mentioned above. Methylene blue undertakes a reversible two-electron, one-proton reduction and has seen the most prevalent use in different kinds of literature. However, the redox reaction is sensitive to changes in $\mathrm{pH}$ and thus, care must be taken when working in conditions where $\mathrm{pH}$ is unknown or varies. On the other hand, ferrocene undergoes reversible, one-electron oxidation to form ferrocenium. However, ferrocenium is prone to nucleophilic attack even by mild nucleophiles like chloride which ultimately can displace the molecule from the nucleic acid. Therefore this particular character limits the utility of ferrocene labels in physiologically relevant solutions.

I used the protocol described in the Methods section to fabricate electrochemical aptamer sensors on gold disk electrode of both MB and Fc modified B23 aptamer and recorded the blank peak signal via SWV. (Figure 3-2). Both two sensor showed correct peak positions in $-2.38 \mathrm{~V}(\mathrm{MB})$ and $2.8 \mathrm{~V}(\mathrm{Fc})$ against $\mathrm{Ag} / \mathrm{AgCl}$ reference electrode.

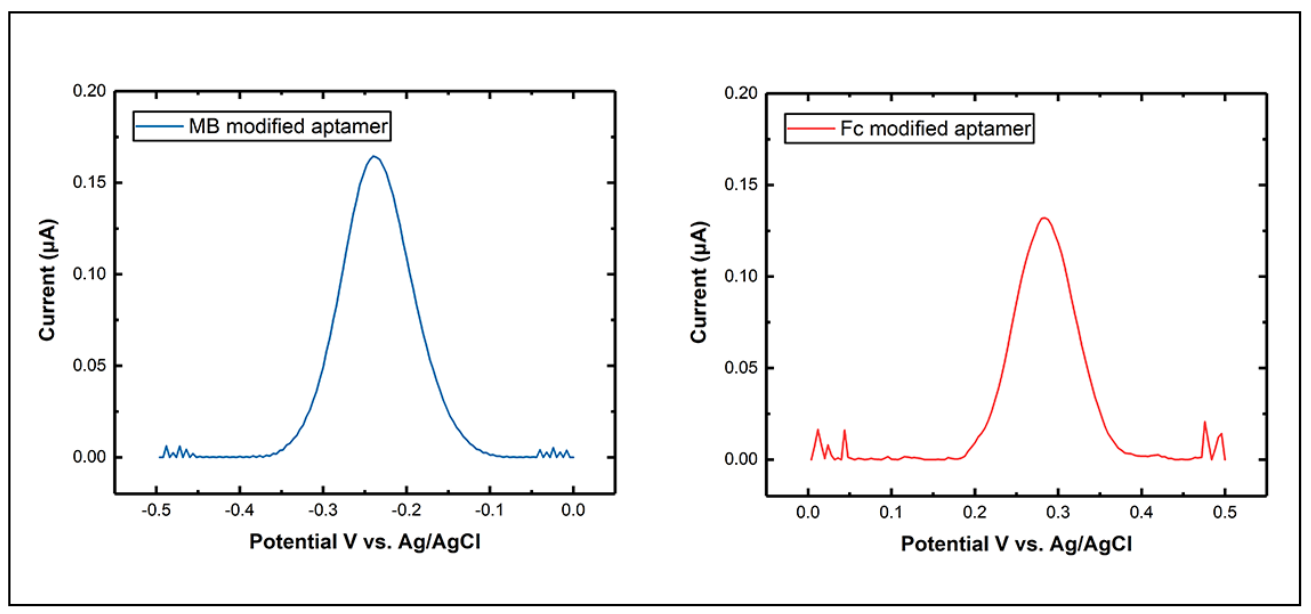

Figure 3-2: Blank SWV signal of MB modified aptamer and Fc modified aptamer on gold disk electrode. The blue curve represents the MB modified aptamer peak which is at $-2.38 \mathrm{~V}$ and the red curve represents the Fc modified aptamer peak which is at $0.28 \mathrm{~V}$. 
I then compared the signal stability by continuing recording their blank SWV signal under 60 mins and the results are showed in Figure 3-3. Fc modified B23 shows a noticeable signal decrease over time while MB remains relatively stable. This phenomenon has also been observed by multiple research groups. I found out this signal decay of Fc label may associate stronger with the number of scans rather than different measurement time.

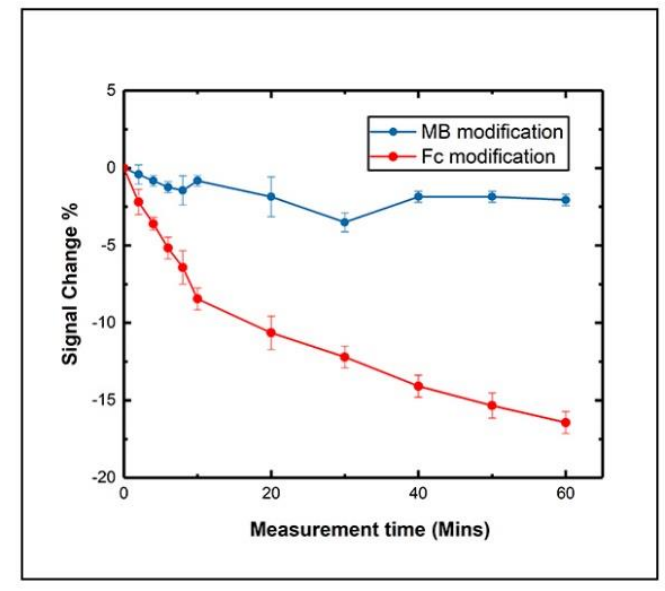

Figure 3-3: Stability comparison between the SWV peak signal generated by MB and Fc modified aptamers. SWV peak height change among 1 hour was used as the reference to compare. An apparent signal decrease can be observed in the Fc modified aptamer signal (red).

Another reason I gave up the usage of $\mathrm{Fc}$ as the labelled probe is that unknown oxidation peak at the similar potential range $(0.25 \sim 0.35 \mathrm{~V})$ was observed when performing SWV measuring on bare gold chip electrode (Micrux, Dropsens). This was an unexpected phenomenon, and I do not have a clear idea of what may trigger this happen. As this peak appears in all of the electrode systems time by time, it's believed that it may be due to the introduction of redox active impurities (Figure 3-4). 


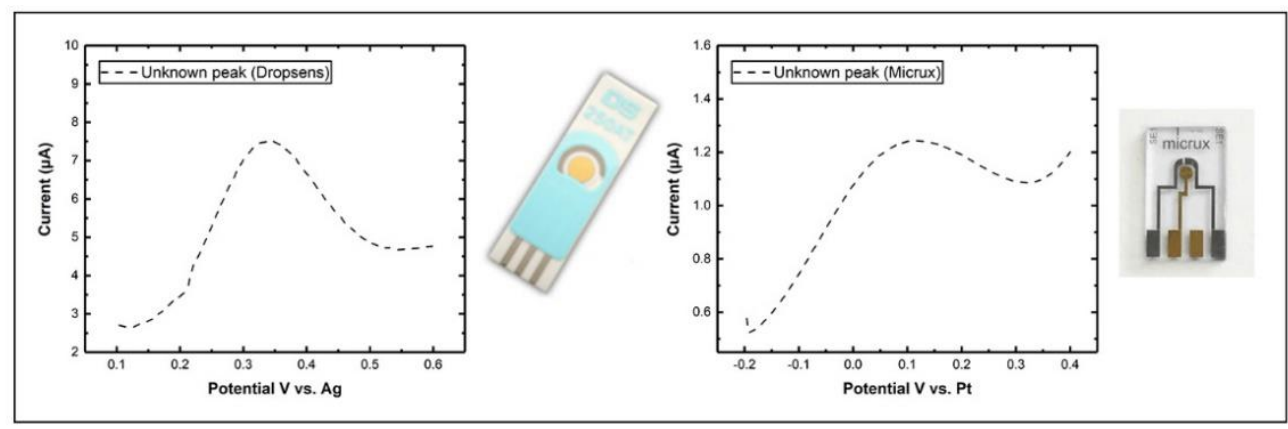

Figure 3-4: Unknown peaks for Fe modified aptamer on chip electrodes Unknown peak appears both on Dropsens and Micrux electrode when scanning SWV in bare electrode under PBS buffer.

\subsection{Co-thiol modification}

Typical E-AB gold electrode surface comprises a mixed monolayer of a thiolmodified aptamer and a thiol spacer such as 6-mercapto-1-hexanol (MCH), mercaptosuccinic acid (MSA), and $\mathrm{EG}_{3}$ (Figure 3-5 A). The usage of these backfilling molecules can form passivating monolayer to stabilise aptamer folding structure and reduce non-specific absorption. Here, I compared three thiol reagents in the traditional gold disk 3-electrode system (Figure 3-5 B).

\subsubsection{MSA, MCH and EG3}

$\mathrm{MCH}$ has been widely reported as the backfilling molecule of E-AB sensor fabrication in the literature $[125,126]$. Early studies showed that the deposition of $\mathrm{MCH}$ could lift non-specifically bound DNA. However, MCH is found to compete with the immobilised thiol aptamer sequence. The $\mathrm{MCH}$ can remove some of the aptamers by substitution at the gold surface. Therefore, EG3, and MSA were also suggested as the alternative backfilling co-thiol molecules as they were reported to be milder compared to $\mathrm{MCH}$ and maintain the high surface density of the recognition element [127].

$\mathrm{MCH}, \mathrm{MSA}$ and $\mathrm{EG}_{3}$ were used as the backfilling molecules to fabricate $\mathrm{LH}$ aptamer sensors. Figure 3-5 C shows the SWV of the blank signals of LH aptamer filled by these co-thiol molecules. These three electrodes were controlled to have 
similar roughness factor and same aptamer immobilisation concentration. Sensor backfilled by $\mathrm{EG}_{3}$ showed the strongest signal when compare to $\mathrm{MCH}$, this may suggest the MCH may compete for some of the LH aptamers off the sensor surface. Sensor backfilled by MSA shows a poor-defined peak signal which it is quite consistent with different batches. This may due to the MSA was not long enough to stabilise the LH aptamer folding and affected the electron transfer of the MB reporter. I chose $\mathrm{MCH}$ as the optimal backfilling molecules as $\mathrm{EG}_{3}$ is quite tricky to handle regarding its smell and storage requirement. (-20 degree).
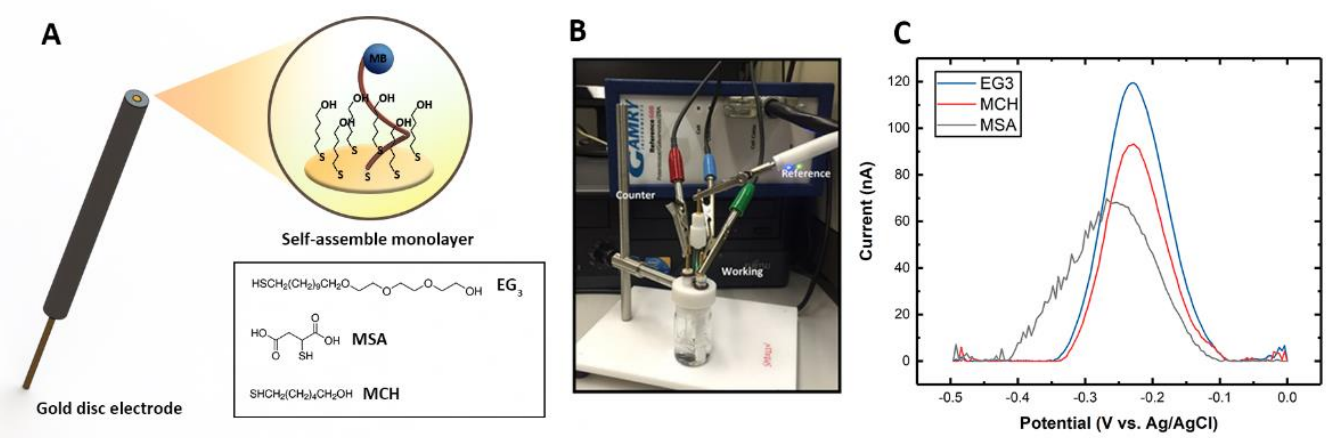

Figure 3-5: Co-thiol modification. (A) $2 \mathrm{~mm}$ Traditional gold disk electrode setup for EAB sensor. MB-modified aptamer is immobilised on the surface and back-filled with cothiol molecules such as EG3, MSA or MCH. (B) Traditional gold disk electrode 3-electrode system setup. $\mathrm{An} \mathrm{Ag} / \mathrm{AgCl}$ reference electrode and platinum counter electrode are used in this system. (C) MB signal comparison among using 3 different co-thiol molecules.

\subsection{Aptamer packing density}

The probe density of $\mathrm{E}-\mathrm{AB}$ sensor is critical for sensor performance. As the basic requirement of the structure-switching $\mathrm{E}-\mathrm{AB}$ sensor is the conformational change upon binding the target, high probe density increases the space hindrance and the degree of change may not be sufficient for qualifying signal readout. On the other hand, low probe density may lead to no readable MB signal. I controlled the packing density of our electrode within the range of $10^{11} \sim 10^{12}$ aptamers $/ \mathrm{cm}^{2}$, which is the optimal self-assemble-monolayer density. I used two steps method to ensure the electrodes I fabricated can meet the probe density requirement:

\subsubsection{Roughness factor (RF) of different electrodes}


First is to select gold disk electrode with consistent quality. Traditional gold disk electrode requires polishing and cleaning before every time of usage. The human errors of handling electrodes could generate issues such as electrode-electrode variants. I applied Roughness factor (RF) as a reference for gold disk electrode handling. RF can be determined using the Eq. (1)

$$
\mathrm{RF}=\frac{\text { Electrode ECSA }}{\text { Electrode Geometric Area }}
$$

(1)

Where ECSA represent the electrochemical active surface area of the electrode, and the ECSA can be determined using Eq. (2):

$$
\mathrm{ECSA}=\frac{\mathrm{Q}_{\mathrm{Au}}}{400 \mu \mathrm{C} \mathrm{cm}^{-2}}
$$

Where $\mathrm{Q}_{\mathrm{Au}}$ is integration of charges under the reduction peak in the $\mathrm{CV}$ of blank gold electrode in $0.5 \mathrm{M}$ sulfuric acid (integral of the area divides scan rate, Figure 3-6 A), $400 \mu \mathrm{C} \mathrm{cm}^{-2}$ is the mean surface concentration of gold atoms based on density and atomic weight of gold.

Gold electrodes with RF in the range of 1.2 to 1.4 were used for all the experiments.

\subsubsection{Packing density calculation}

Second is to calculate the packing density of every electrode after fabrication. The aptamer packing density can be calculated using Eq. (3):

$$
\text { Packing density }=\frac{\mathrm{Q}_{\mathrm{MB}}}{\mathrm{nF}(\mathrm{ECSA})}
$$

(3)

Where $\mathrm{Q}_{\mathrm{MB}}$ is the integration of charges under the reduction peak in the $\mathrm{CV}$ of aptamer-modified electrode in PBS buffer (integral of the area divides scan rate, Figure 3-6 B) 
As I used the range of $10^{11} \sim 10^{12}$ aptamers $/ \mathrm{cm}^{2}$ for our packing density reference, which equals approximately 550 650 pmoles $/ \mathrm{cm}^{2}$. I used the methods described to ensure every hand fabricated electrode can meet the requirements.

A

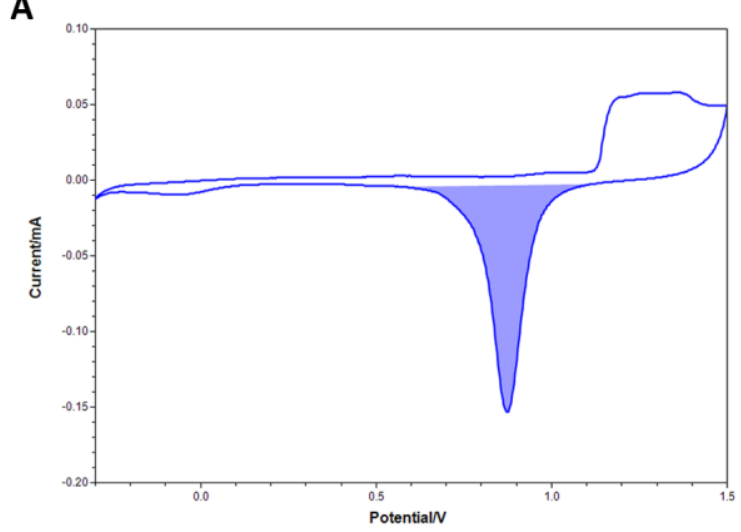

B

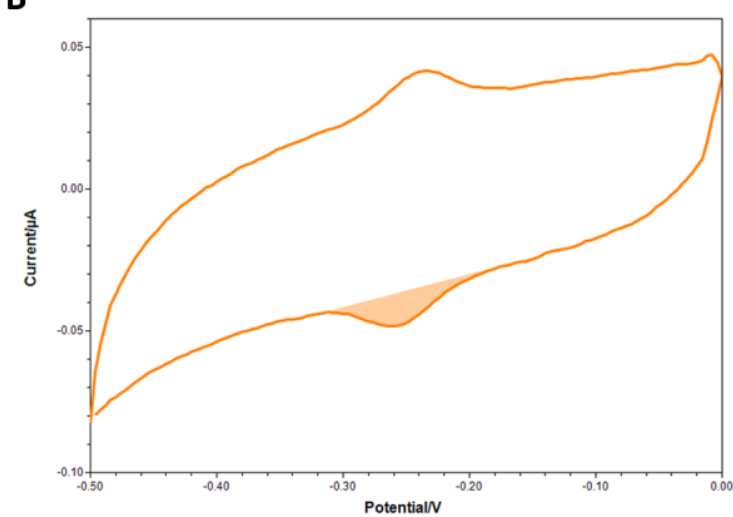

Figure 3-6: Methods for calculating the probe density. (A) CV scan of a clean gold electrode in $0.5 \mathrm{M}$ sulfuric acid solution. The blue area indicates the integral of the gold reduction peak and the value of the integral can be calculated by the potentialstat programme. $\mathrm{Q}_{\mathrm{Au}}$ can be calculated from the integral and ECSA can be calculated according to Eq. (2) (B) CV scan of an aptamer-immobilised electrode in PBS buffer. From the orange integral, $Q_{\text {мв }}$ can be calculated, and the aptamer packing density can be then calculated using Eq. (3).

\subsection{Electron transfer kinetic characterisation}

After the optimisation of the usage of redox label, co-thiol molecule and aptamer probe packing density, most of the parameters of the LH electrochemical assay were confirmed. I start to look into the more theoretical characterisation of the whole system such as the electron transfer kinetic calculation. This can give me more information about whether the current setup is generating the correct signal output.

\subsubsection{Coefficient constant and charge transfer rate constant calculation}

Useful information involving electrochemical mechanism usually can be acquired from the relationship between the peak current and scan rate. Therefore, the influence of scan rate on the oxidation and reduction peak current of MB-modified LH aptamer sensor was investigated in detail. According to the method described 
by Laviron [128], the charge transfer coefficient, $\alpha$, and the apparent heterogeneous charge transfer rate constant, $k s$, for the electron transfer between an gold electrode and a surface-confined redox couple of $\mathrm{MB}$ can be evaluated in Cyclic Voltammetry $(\mathrm{CV})$ by the variation of the anodic and cathodic peak potentials with the logarithm of scan rates. Figure 3-7 A shows the Cyclic Voltammogram of MBmodified LH aptamer immobilised electrode in PBS buffer under different scan rates (from $0.03 \mathrm{~V} / \mathrm{s}$ to $0.2 \mathrm{~V} / \mathrm{s}$ ). The plot of the peak current vs scan rate is linear over the whole range of scan rate studied (Figure 3-7 B), which this indicates the electron transfer is under a typical surface controlled model - evidence of the successfulness of immobilisation of MB-modified LH aptamer. The linear relationship between the peak current and the scan rate was obtained with the linear regression equation Eq. (4):

$$
\text { Ipa }=239.87 \mathrm{v}-5.57
$$

where the $\mathrm{v}$ represents the scan rate $\left(\mathrm{r}^{2}=0.998\right)$.

Using this equation together with Laviron's equation [128] Eq. (5):

$$
\mathrm{Ipa}=\frac{\mathrm{n}^{2} \mathrm{~F}^{2} \mathrm{AT} \mathrm{s}}{4 \mathrm{RT}}
$$

Where $\mathrm{n}$ is the electron transfer number $(\mathrm{MB}=2), \mathrm{A}$ is the Electrochemical Active Area, $\mathrm{F}$ is the Faraday constant, $\mathrm{R}$ is the gas constant, $\mathrm{T}$ is the temperature.

Therefore, the adsorption capacity $\left(\mathrm{T}_{\mathrm{s}}\right)$ can be calculated as $694 \mathrm{pmoles} / \mathrm{cm}^{2}$, which consistent with the packing density I optimised before.

Also, Figure 3-7 C shows the relationship between oxidation peak potential (Ep) and the logarithm of scan rate ( $\log \mathrm{v})$. Epa (Oxidation peak potential) and Epc (Reduction peak potential) were plotted separately to give two branches. It can be seen that at high scan rates, the plotting of Epa and Epc vs log v produces a linear line with linear regression equation Eq. (6) $\left(r^{2}=0.997\right)$ and Eq. (7) $\left(r^{2}=0.992\right)$ :

$$
\text { Epa }=0.19 \log \mathrm{v}-2.82
$$

$$
\mathrm{Epc}=-0.23 \log \mathrm{v}-2.07
$$

These equations, together with Laviron's equation Eq. (8) and Eq. (9), 


$$
\mathrm{Epa}=\mathrm{a}+\frac{2.303 \mathrm{RT}}{(1-\alpha) \mathrm{nF}} \operatorname{logv}
$$

(8)

$$
\mathrm{Epc}=\mathrm{b}-\frac{2.303 \mathrm{RT}}{\alpha \mathrm{NF}} \operatorname{logv}
$$

(9),

As the $\mathrm{n}$ for MB equal 2, the coefficient constant $\alpha$ can be calculated as 0.45 . For the apparent heterogeneous charge transfer rate constant, $k s$, can be calculated using Eq. (10):

$$
\log k s=\alpha \log (1-\alpha)+(1-\alpha) \log \alpha-\log \frac{R T}{n F v}-\frac{\alpha(1-\alpha) n F \eta}{R T}
$$

Where $\eta$ is the overpotential $=\mathrm{Ep}-\mathrm{E} 0$, and $\mathrm{E} 0=(E p a+E p c) / 2)$. Therefore, the ks of the MB-modified aptamer can be calculated as around $20 \mathrm{~s}^{-1}$, which is consistent with most of the MB tethered oligos published in the literature [129].
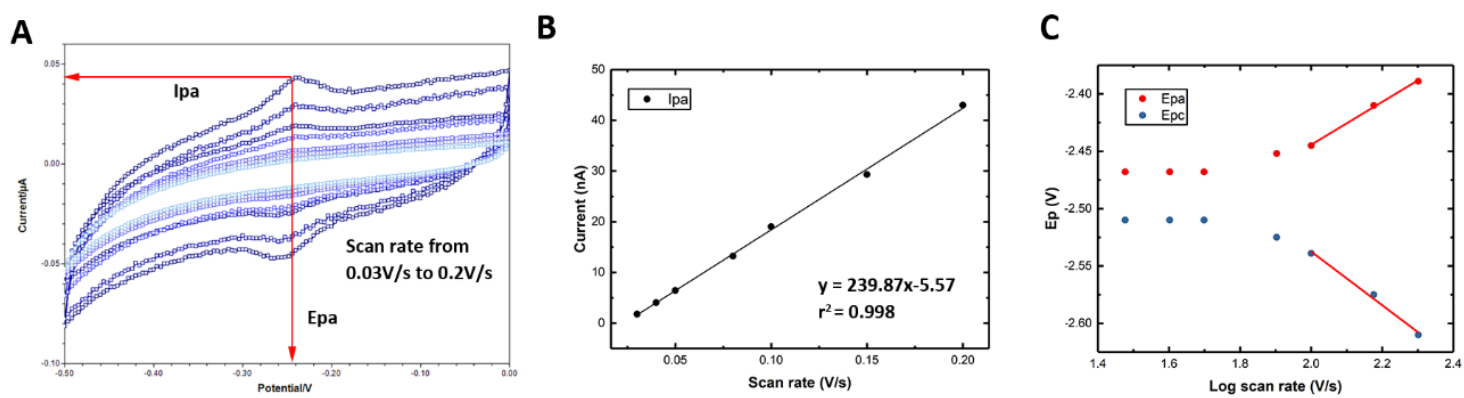

Figure 3-7: Effect of scan rate. (A) Cyclic Voltammogram for MB-modified LH aptamer immobilised electrode in PBS buffer under scan rates $(0.03,0.04,0.05,0.08,0.1,0.15$ and $0.2 \mathrm{~V} / \mathrm{s}$ ). The red arrows point out the oxidation peak current (Ipa) and oxidation peak potential (Epa). (B) Plot Ipa vs. Scan rate. Linear relationship indicates the electron transfer is under a surface-controlled mechanism. (C) Plot Ipa vs. log v. At high scan rates, both Epa and Epc exhibit linear relationship vs. $\log \mathrm{v}$.

\subsection{Signal response upon binding $\mathrm{LH}$}

The signal response upon the binding of LH was characterised via multiple electrochemical techniques including SWV and ACV. By comparing the sensitivity 
and feasibility of using all these methods for quantification, I can find out the best suitable one for the final sensing platform application.

\subsubsection{SWV}

The signal in E-AB sensors is driven by binding-induced changes in the rate of electron transfer from the attached redox reporter. Square wave voltammetry is typically employed to convert this change in electron transfer rates into a change in observed current.

In this method, I apply a "staircase" potential waveform and measure the Faradic current at the end of each square pulse. A current vs potential voltammogram is generated from this by taking the difference between each subsequently measured current. I first optimised the frequency for the measurement, as shown in Figure 3-8, the LH aptamer sensor shows a "signal on" response at the range of $0 \sim 10 \mathrm{~Hz}$ and a "signal off" response at the range of $10 \sim 500 \mathrm{~Hz}$ (the highest frequency our potentialstat can achieve).

However, in practice I realised the "non-response" frequency sometimes shift towards lower frequency range and for our LH sensor, it's difficult to use the "signal on" range to quantify LH concentration as in low frequency, the peak's shape is not very clear which makes the potentialstat's software sometimes cannot initial auto peak function. Manual peak will introduce errors. Therefore, I chose $100 \mathrm{~Hz}$ at the "signal off" range as our optimal frequency and performed titration study using this setup. 


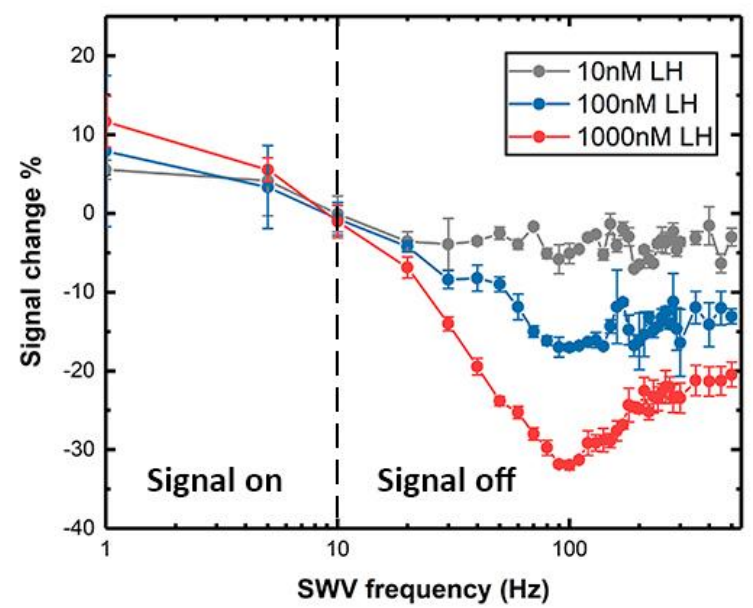

Figure 3-8: Signal response change under different SWV frequency. MB peak signals of the LH aptamer was recorded under scans in different SWV frequency setting. I applied three concentrations $(10,100$ and $1000 \mathrm{nM})$ of $\mathrm{LH}$ to investigate the signal change.

Using $100 \mathrm{~Hz}$ as the optimal SWV frequency for LH aptamer sensor, I tested the dose-response of the LH aptamer under this sensing format against LH and FSH. Figure 3-9 A shows the peak current decrease trend upon adding different concentration of LH in the buffer and Figure 3-9 B shows a dose-response relationship. After fitting to a Hyperbl model, the $\mathrm{K}_{\mathrm{D}}$ estimated is $144 \mathrm{nM}$ and the linear range is from $5 \mathrm{nM}$ to $100 \mathrm{nM}$.

One of the disadvantages of "signal off" sensing format is the limited signal gain. I found out the LH sensor reach the saturation around $2000 \mathrm{nM}$ and the maximum gain is around $40 \%$. The narrow linear range of the current sensor format is not wide enough to cover the pulsatility range. Therefore, further optimisation of the sensor is required to increase the signal gain and broaden the linear range of the sensor. 
A

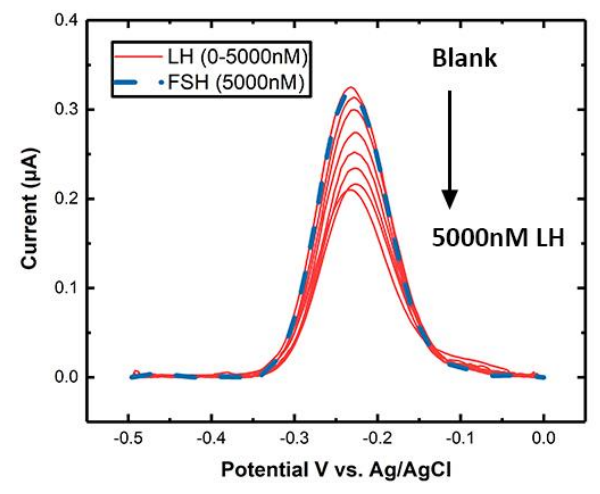

B

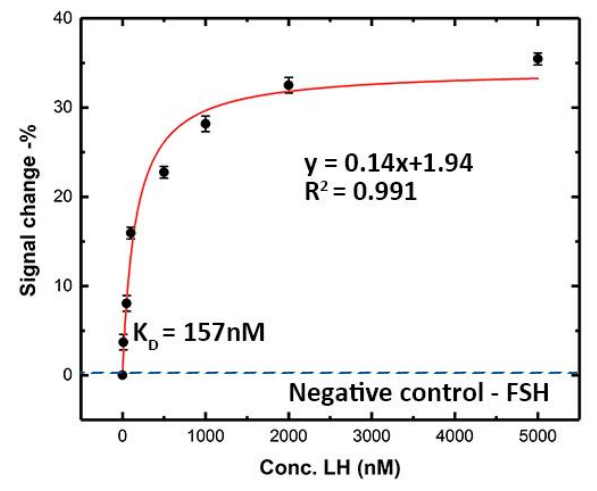

Figure 3-9: SWV dose response. (A) Square-wave voltammogram of gold disk LH aptamer sensor. All the curves were obtained by performing SWV scan from $-0.5 \mathrm{~V}$ to $0 \mathrm{~V}$, with $100 \mathrm{~Hz}$ frequency and $25 \mathrm{mV}$ Amplitude. 8 concentrations of LH were used: $0,10,50$, 100, 200, 500, 1000 and 2000nM. (B) Dose-response curve using SWV methods. The "-\%" of the signal change indicates the change is a "signal off" model. The $\mathrm{K}_{\mathrm{D}}$ is estimated to be $144 \mathrm{nM}$ and the linear range is from $10 \mathrm{nM}$ to $100 \mathrm{nM}$

\subsubsection{ACV}

To double confirm the E-AB conformational change signalling mechanism, I further applied ACV to investigate the dose-response of the sensor. Recently there has been literature pointed out using ACV instead of SWV may achieve a bettersignal gain and reach a better Limit of Detection (LoD) for ampicillin detection in a complex sample [130]. Figure 3-10 shows the original data obtained from the potentialstat for the titration study. Unfortunately, no matter how I optimise the experimental setting, the data still being very noisy. Therefore I have to use external peak analysis by Origin to extract the peak height information (Figure 3-11 A). In this experiment, all the LH concentration arrangement and sensor fabrication was exactly the same as the SWV experiment. As can be seen in Figure 3-11 B, I plotted the dose-response curve and fitted under a hyperbl model. The $K_{D}$ estimated is $540 \mathrm{nM}$, about 2.5 times less than the $K_{D}$ measured by SWV. Which this result was controversial against the ampicillin paper (they stated ACV could achieve higher sensitivity in their condition). However, I noticed the signal gain increased when I apply ACV for the measurement slightly. Even this, I still have the disadvantage of long data processing time required for $\mathrm{ACV}$. 


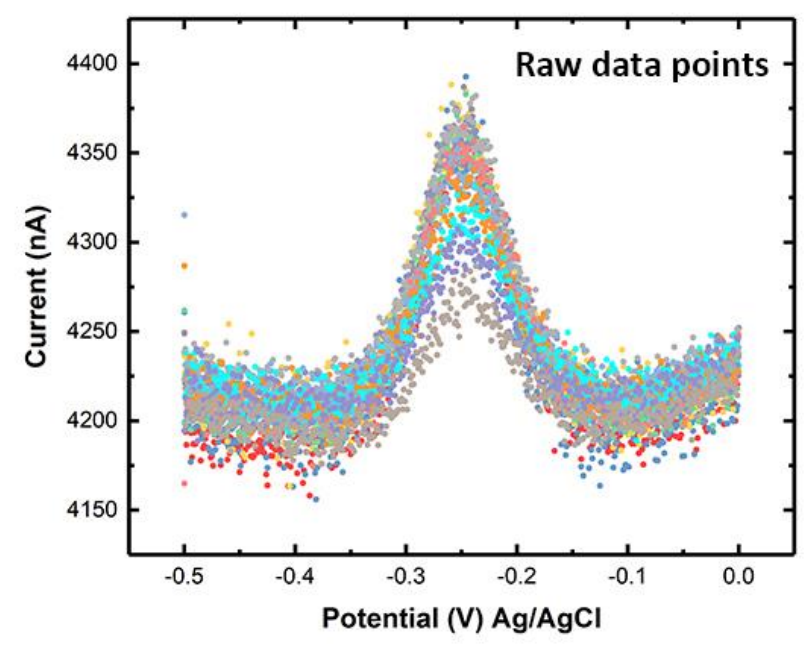

Figure 3-10: ACV raw data point. Raw ACV data obtained by scanning from -0.5 to 0 $\mathrm{V}$. The peaks here are scans of different concentration LH in PBS buffer on the LH aptamer sensor.

\section{A}

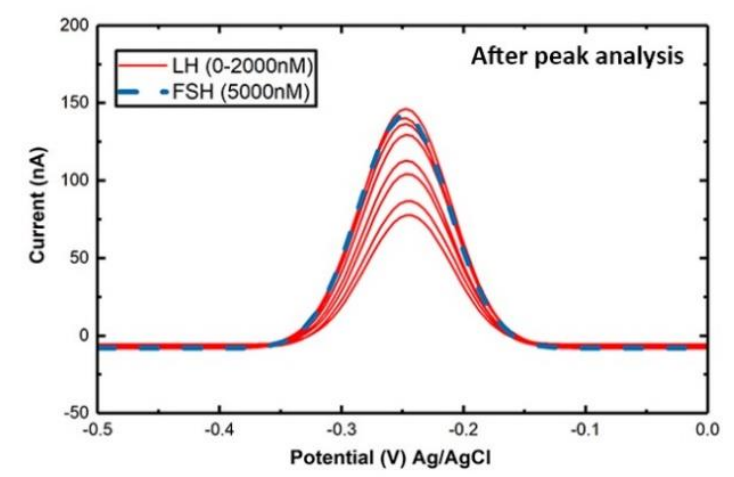

B

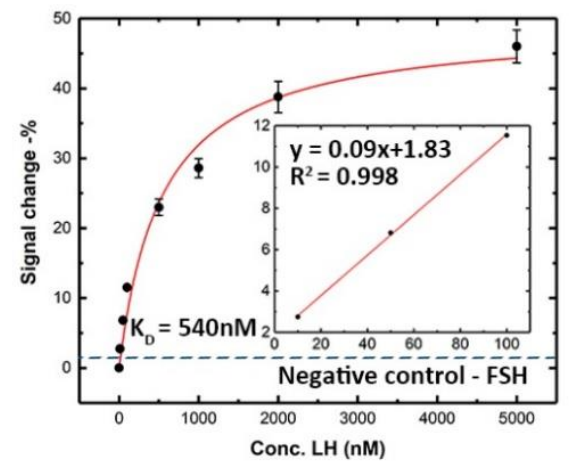

Figure 3-11: ACV dose response. (A) Processed ACV data. I used the Origin's peak analysis function to calculate the peak highs. Negative control was 5000nM FSH. (B) Doseresponse curve using SWV methods. The "-\%" of the signal change indicate the change is a "signal off" model. The $\mathrm{K}_{\mathrm{D}}$ is estimated to be $540 \mathrm{nM}$ and the linear range is from $10 \mathrm{nM}$ to $100 \mathrm{nM}$. 


\subsection{Chapter summary}

In this chapter, I applied traditional gold disk electrode system to optimise the basic parameters for the LH electrochemical assay. The reason of that is compared to some of the other disposable electrode platforms, gold disk electrode shows more rigid and stable performance as the bulk polycrystalline gold layer are usually thicker than most of the chip-based electrode, this contributes to a more stable Selfassembly monolayer immobilisation [131].

The optimal LH electrochemical assay parameters were confirmed after testing the usage of different redox probes (Figure 3-1 to Figure 3-3), co-thiol molecules (Figure 3-5). It is unexpected that I encounter Fc signalling issue on the screenprinted electrode (Figure 3-4). I tested all of those electrodes straight out of the box so the electrochemical impurities should not be introduced from our lab reagents. However, positive potential range where Fc posited indeed covers quite many electrochemical components therefore I believe the choice of using MB is much better. The electrochemical property of the LH aptamer sensor was characterised by performing different CV scan rate, the electron transfer constant was calculated and it was consistent with surface immobilised redox species which provides evidence of the successful immobilisation of the LH aptamer.

The second aim was achieved by measuring the dose-response using both SWV and ACV electrochemical methods. From the result obtained by using SWV, the $\mathrm{K}_{\mathrm{D}}$ estimated was stronger than the ACV (Figure 3-9 and Figure 3-11). However, using ACV for the measurement seems can provide a larger signal gain. I chose SWV as our final assay method as the measurement time is much faster than ACV. Confirming all the assay parameters provide us a useful reference for the further customisation of the sensing platform.

\subsection{Materials and Methods}

\subsubsection{Materials and equipment}

All chemicals and reagents were of analytical laboratory grade or higher. For the Ferrocene labelling, EDC and sulfo-NHS were purchased from Sigma-Aldrich. Thiol and amine modified B23 was custom synthesized in Integrated DNA 
Technology. Methylene blue modified B23 was custom synthesized in Biomer.net. Electrode set (gold disk electrode, $\mathrm{Ag} / \mathrm{AgCl}$ reference electrode, platinum counter electrode) and polishing pads/solutions were purchased from IJ Cambria Scientific. The potentialstat used for CV, SWV measurement was Emstat blue from palmsens. The potentialstat used for ACV measurement was compactstat from Ivium Technologies.

\subsubsection{Chemical modification of B23 with redox group}

N-Hydroxysuccinimide (NHS) and 1-Ethyl-3-(3-dimethylaminopropyl) carbodiimide (EDC) were used to conjugate the redox reporter Ferrocene (Fc) onto B23 in order to produce the electrochemical molecular beacon. Briefly, $1 \mathrm{mM}$ of EDC, $5 \mathrm{mM}$ of NHC were added to a PBS solution contained Ferrocenecarboxylic acid and amino/ thiol modified B23 with a moles ratio of 50:1. The mixture was incubated overnight on the shaker at room temperature. NAP-5 column was used to purify the Fc-B23. The concentration of the resulted product was evaluated by UVvis spectrophotometry. Since the ferrocene label has absorption in A260 and A450, Calibration was made by a dilution series of the Ferrocenecarboxylic acid. The FcB23 was saved in $-20^{\circ} \mathrm{C}$ freezer for the next modification.

\subsubsection{Electrochemical experiment setup}

3 -electrode system was used as the initial design of the electrochemical sensor. This system consists of a $2 \mathrm{~mm}$ gold working electrode, a $0.5 \mathrm{~mm}$ platinum counter electrode, an $\mathrm{Ag} / \mathrm{AgCl}$ reference electrode and a $10 \mathrm{~mL}$ electrochemical cell (Figure 3-2 B). CV, SWV were measured using this 3-electrode system under Emstat. ACV was measured under Compactstat.

\subsubsection{Electrode cleaning}

Different methods were tested for the electrode cleaning. The major 3 steps of the cleaning include solution cleaning, polishing, and electrochemical cleaning. Water, piranha solution, and a mild version of piranha solution $\left(30 \% \mathrm{H}_{2} \mathrm{O}_{2}: 30 \% \mathrm{NH}_{3}\right.$ : water $=1: 1: 5)$ were tested for the solution cleaning. 3 mins polishing with $1 \mu \mathrm{m}$ 
micro cloth suspension in diamond and 3 mins polishing with $0.05 \mu \mathrm{m}$ micro cloth suspension in alumina were used after the solution cleaning. For the electrochemical cleaning, methods include performing a $0.5 \mathrm{M} \mathrm{H}_{2} \mathrm{SO}_{4}$ cleaning via cyclic voltammetry (oxidation $2 \mathrm{~V}$ for $5 \mathrm{~s}$, reduction $-0.35 \mathrm{~V}$ for $10 \mathrm{~s}$ followed a full scan from -0.35 to $1.5 \mathrm{~V}$ at a scan rate of $0.1 \mathrm{Vs}^{-1}$ and sample interval of $10 \mathrm{mV}$ until the gold identical peaks can be clearly observed), and performing both basic and acidic cleaning using $0.5 \mathrm{M} \mathrm{NaOH}$ and $0.5 \mathrm{M} \mathrm{H}_{2} \mathrm{SO}_{4}$ under similar condition as mentioned above. The cleaning cycle was terminated by a clear observation of typical cyclic votammogram ( 3 identical oxidation peaks) for a clean gold electrode.

\subsubsection{Aptamer immobilisation}

The immobilisation of the Fc-modified and MB-modified B23 aptamer onto the gold electrode was achieved by the interaction between the modified thiol group on one end of the B23 and the gold surface. Before incubation of the modified aptamer with the previously cleaned electrode, $2 \mathrm{ul}$ of $10 \mathrm{mM}$ TCEP was added to the aptamer solution and incubate 1 hour at $4{ }^{\circ} \mathrm{C}$ fridges to reduce the disulfide bond. The gold electrode was immersed into the resulted product (200nM Fc-B23) for overnight incubation at room temperature for the formation of DNA self-assembled monolayers (SAM). 200ul of $2 \mathrm{mM}$ Mercaptosuccinic acid (MSA) or $\mathrm{EG}_{3}$ or $\mathrm{MCH}$ was then used as a blocking reagent to block the unoccupied binding sites of the gold surface for 1 hour. The electrode was then saved in the binding buffer (PBS + $5 \mathrm{mM} \mathrm{MgCl}$ ) with parafilm sealed before the sensing measurement. All of the measurements were performed with the system mentioned above.

\subsubsection{Cyclic Voltammetry}

For the Fc-modified aptamer, the $\mathrm{CV}$ scan potential was from 0 to $0.6 \mathrm{~V}$. For the MB-modified aptamer, the CV scan potential was from -0.5 to $0 \mathrm{~V}$. The scan rate study was performed only using MB-modified aptamer, under a scanning potential ranging from -0.5 to $0 \mathrm{~V}$. Seven scan rates were used: $0.2,0.15,0.1,0.08,0.05,0.04$, and $0.03 \mathrm{~V} / \mathrm{s}$. 


\subsubsection{Square-wave Voltammetry}

For the Fc-modified aptamer, the SWV scan potential was from 0 to $0.6 \mathrm{~V}$. For the MB-modified aptamer, the SWV scan potential was from -0.5 to $0 \mathrm{~V}$. Other SWV parameters were $25 \mathrm{mV}$ amplitude, $100 \mathrm{~Hz}$ frequency, and $4 \mathrm{mV}$ steps.

\subsubsection{Alternative Cyclic Voltammetry}

For the MB-modified aptamer, the ACV scan potential was from -0.5 to $0 \mathrm{~V}$. Other ACV parameters were $25 \mathrm{mV}$ amplitude, $100 \mathrm{~Hz}$ frequency, and $0.1 \mathrm{~V} / \mathrm{s}$ scan rate. 


\section{CHAPTER 4 | Rapid prototyping devices for the electrochemical assay}

In the previous chapter, I demonstrated how I adopt the aptamer probe into an electrochemical-based assay format on standard gold disk electrode system. While I have this potential tool to perform LH assay, our addressability remains limited to a manual single point measurement in the laboratory setting and large electrode size. In this chapter, I focus on illustrating how I minimise the electrode and use advance rapid prototyping technologies such as $3 \mathrm{D}$ printing and $\mathrm{CNC}$ to assist the design on finalising a whole user-friendly and clinical feasible platform to perform LH pulsatility measurement. I show the proof of concept for a tube-based continuous sensing platform and develop a novel automatic 96 well plate assay system using an open-source liquid handling robot called AELECAP - Automated ELEctroChemical Aptamer Platform.

Keywords: Electrode roughening, rapid prototyping, 3D printing, automation

\subsection{Background}

The goal for all the aptamer-based detection methods discussed is to create a complete functional biosensing system for biomarker detection. The aptasensors usually need to be integrated into an appropriate system. These biosensing systems usually consist 3 key components: Sample preparation/collection device, core recognition element, and signal readout device.

Rapid prototyping techniques such as $3 \mathrm{D}$ printing provides a perfect way to prototype integrated system. In our lab, wegenerated aptamer-based sensing system for malaria diagnostic based on different designs [132]. Thanks to the nature of aptamer for easy modification, I believe adopting these technologies can develop an integrated sensing system for LH pulsatility measurement. Therefore, this chapter aims to utilise rapid prototyping technologies to prototype different devices base on the aptamer and electrochemical assay developed for LH measurement. As well as the charaterisation of their performance. 
I have two strategies for designing medical device prototypes. The first one is to design an integrated liquid handling platform for automatic measurement on multiple samples. The advantage of this is it can be directly validated on clinical samples and compare the performance with the clinical automated immunoassay platform. The second strategy is to design a continuous sensing platform which can be used as a proof-of-concept prototype for the ultimate goal of the sensing the LH pulsatility in a real-time format.

\subsection{Electrode optimisation}

\subsubsection{Issues of commercialised electrodes}

Minimisation of the electrode has been done to improve the feasibility of the device by optimisation of different choice of electrodes and customise electrodes via multiple electrode fabrication methods. I have been tried using commercialised chip-based disposable electrodes to repeat experiments I did at Chapter 3 for doseresponse characterisation. However, there were some critical issues for those electrodes: The first attempt was using Dropsens electrode. Dropsens provides screen-printed electrodes with different materials. The one I selected for our application is AT250, which present a gold working electrode printed with hightemperature ink, a platinum counter electrode and a silver reference electrode (Figure 4-1 A, right). AT250 provides a relatively large working electrode area (4mm diameter) therefore I thought I could observe a higher current signal when compared to the gold disk electrode I used. However, I failed to obtain any MB signals both using SWV and CV to interrogate (Figure 4-1 A, left). I occasionally see in SWV, a small peak appears at the position of $-0.1 \mathrm{~V}$, but this will disappear with multiple SWV scans. This may occur due to electrochemical active impurities (same issue mentioned in Section 3.1.2). Then I tried multiple cleaning protocols including ethanol, electrochemical polishing, and piranha solution but the results were the same. Intensive electrochemical cleaning and piranha solution cleaning even destroyed the gold metal layer. A similar situation happened in my next attempt, Micrux electrode, which is a thin-film mini electrode present a $1 \mathrm{~mm}$ diameter gold working electrode, platinum counter and a reference electrode (Figure 4-1 B, right). As can be seen in the Figure 4-1 B, left, I failed to obtained MB peak signal of LH-aptamer immobilised micrux electrode measured by both SWV and CV. 
One assumption is no MB-modified LH aptamer presented on these electrodes therefore no MB signal can be observed. To verify this, I utilised the malaria aptamer 2008s developed by our group as it can generate colourimetric response via enzymatic assay called APTEC (APtamer-Tethered Enzyme Capture) [133]. I immobilised a thiol-modified 2008s aptamer onto Dropsens, Micrux and gold disk electrode, blocked with $\mathrm{MCH}$ and performed APTEC to see whether they can all generate a colourimetric response. As shown in Figure 4-1 C, only gold disk electrode showed a response. This indicates the thiol-modified aptamer only be successfully modified on the gold disk electrode but not Dropsens and Micurx electrode. 

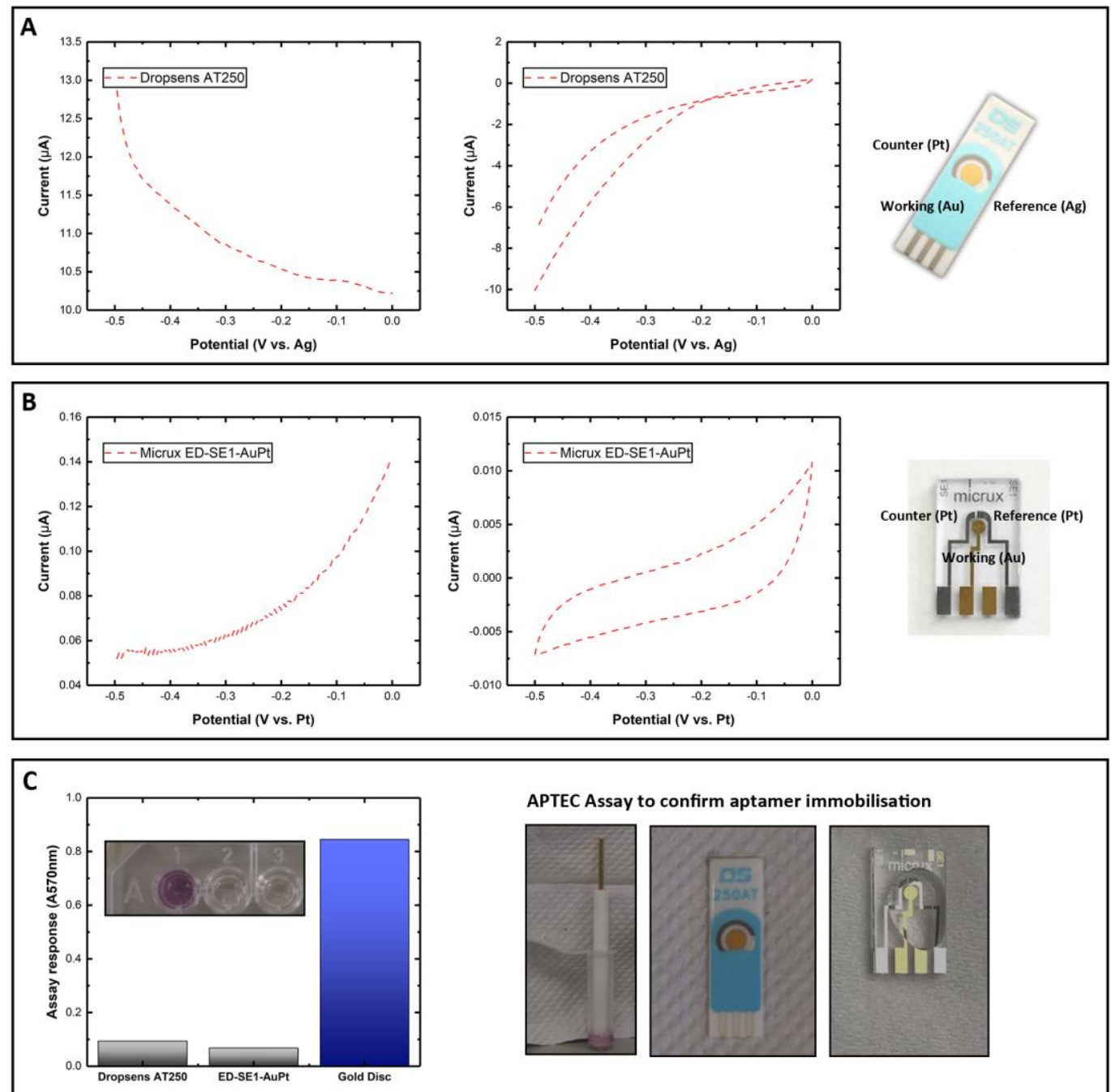

Figure 4-1: Investigation of Dropsens and Micrux electrode. (A) Cyclic Voltammogram and Square-wave Voltammogram of LH aptamer-modified Dropsens AT250 electrode. A small peak appeared at the Square-wave Voltammogram about $-0.1 \mathrm{~V}$ but this is regarded as impurity peak. (B) Cyclic Voltammogram and Square-wave Voltammogram of LH aptamer-modified Dropsens ED-SE1-AuPt. (C) APTEC assay results.

\subsubsection{Customised electrodes}

I further investigate the possibility of using customised electrode instead of commercialised one. Because I can control better of the fabrication parameters and optimised the process. I used physical deposition and chemical deposition methods to fabricate two kinds of the gold electrode. The first one is a gold sputtering 
electrode. As can be seen in the Figure 4-2 A, right, the electrode I fabricated is a micro-needle electrode with three gold electrodes pad and one silver electrode pad. I believe by increasing the working electrode area, MB signal should be able to be detected. Figure 4-2 A, left shows the SWV and CV of LH aptamer-immobilised microneedle electrode in PBS. Clear MB peak can be observed in both voltammograms. I also fabricated another electrode by using chemical deposition. Figure 4-2 B shows the SWV and CV of LH aptamer-immobilised chip electrode in PBS. Similar peaks can be observed as the microneedle electrode.
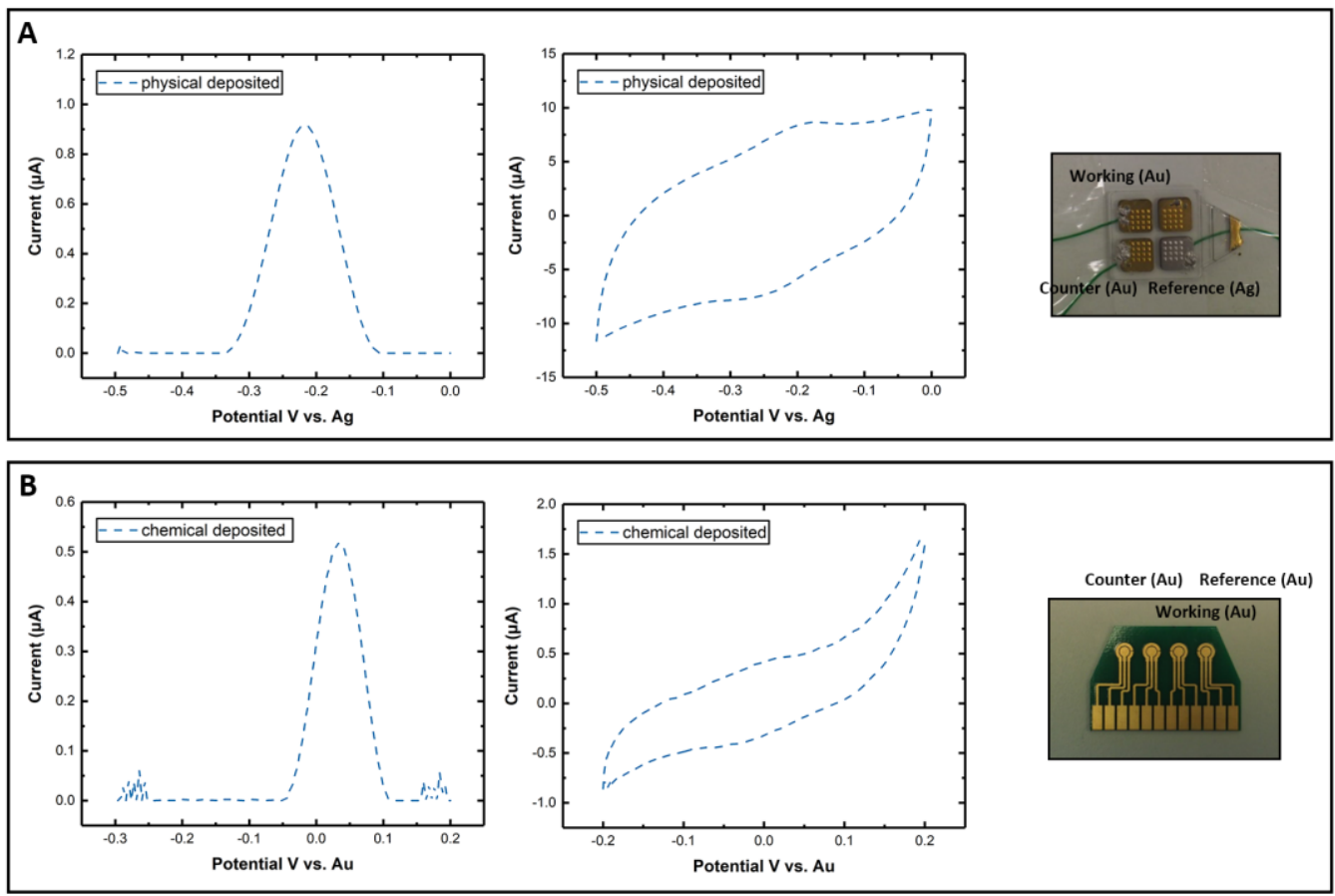

Figure 4-2: Investigation of physical deposited and chemical deposited electrode. (A) Cyclic Voltammogram and Square-wave Voltammogram of LH aptamer-modified Dropsens AT250 electrode. A small peak appeared at the Square-wave Voltammogram about $-0.1 \mathrm{~V}$ but this is regarded as impurity peak. (B) Cyclic Voltammogram and Squarewave Voltammogram of LH aptamer-modified Dropsens ED-SE1-AuPt.

\subsection{Golden wire electrode}

\subsubsection{Design and cleaning}

Another attempt for me to generate suitable minimised electrode system is microwire. These wires are different metals with diameters ranging from $0.3 \mathrm{~mm}$ to $1 \mathrm{~mm}$. For each electrode, I used a protective design illustrated in Figure 4-3 A. In 
this design, about $5 \mathrm{~mm}$ metal wire is exposed for surface modification. The other part of the wire is wrapped with non-conductive materials such as a heat-shrinkable tube or PVC tape and sealed with non-conductive epoxy. This provides a consistent contact electrode area when dip the wire into solution. The length of the wire can be adjusted according to the application. A 3-wire-electrode system were used to test the basic performance of the wire electrode Figure 4-3 B. Before electrochemical cleaning, there werestrange oxidation and reduction peaks can be seen around $0.5 \mathrm{~V}$ when performing $\mathrm{CV}$ scan in $0.5 \mathrm{M}$ sulfuric acid. After multiple $\mathrm{CV}$ scans from -0.3 to $1.5 \mathrm{~V}$ in $0.5 \mathrm{M}$ sulfuric acid (typical electrochemical cleaning protocol), the peak disappeared and the typical clean gold electrode voltammogram can be obtained (Figure 4-3 C). I also performed CV and SWV after I immobilised the LH aptamer. As can be seen in Figure 4-3 D and E, the MB peak can be easily seen. This means the immobilisation was successful.
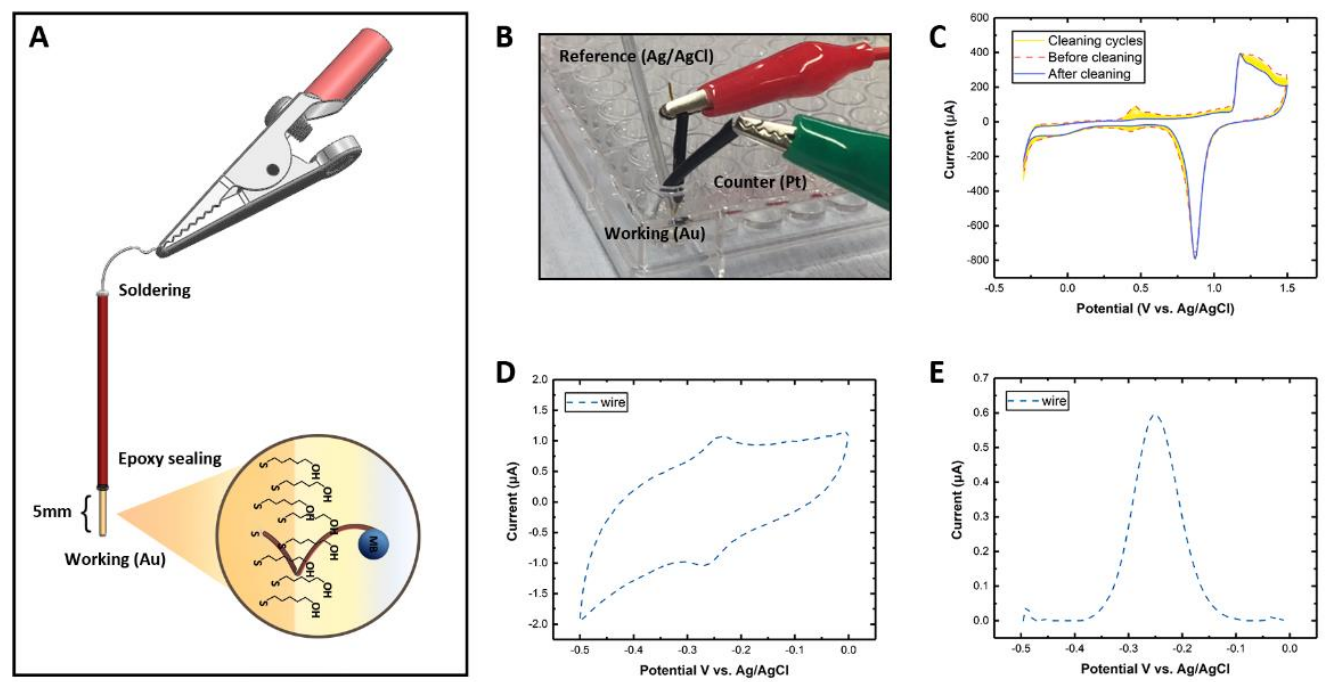

Figure 4-3: Investigation of the wire electrode. (A) Design of the wire electrode. MBmodified LH aptamer is immobilised on the $5 \mathrm{~mm}$ wire surface. Heat-shrinkable tube and PVC tape with epoxy sealing are used to insulate the wire and maintain a certain contact area for the measurement. The other end of the electrode is soldered to the metal wire for connection. (B) Picture of 3-wire-electrode-system. Here I use the 96-well plate as the container. (C) Cyclic Voltammogram of multiple scans from $-0.3 \mathrm{~V}$ to $1.5 \mathrm{~V}$ in $0.5 \mathrm{M}$ sulfuric acid. The impurity (peaks around $0.5 \mathrm{~V}$ ) can be removed after multiple cycles. (D \& E) Cyclic Voltammogram and Square-wave Voltammogram of LH aptamer-modified wire electrode. 


\subsubsection{Roughening of the wire electrode}

Electrochemical roughening can increase the electrochemically active area. One literature mentioned roughening of the electrode may increase the signal gain [123]. To further investigate the effect, I performed electrochemical roughening and applied $5000 \mathrm{uM}$ of $\mathrm{LH}$ to compare the signal response before and after the roughening step. The roughening can be confirmed via comparing the Cyclic Voltammogram before and after roughening in $0.5 \mathrm{M}$ sulfuric acid. As shown in Figure 4-4 A, the reduction and oxidation peak of the gold electrode was increased after roughening. I also performed signal response study on adding the same amount of LH to the wire electrode using setup illustrated in the last section. Figure 4-4 B $\boldsymbol{\&} \mathbf{C}$ shows the difference of signal response upon adding $2 \mu \mathrm{M} \mathrm{LH}$ for normal wire electrode and the roughened electrode. There is a noticeable gain increased as shown in the Figure 4-4 C.
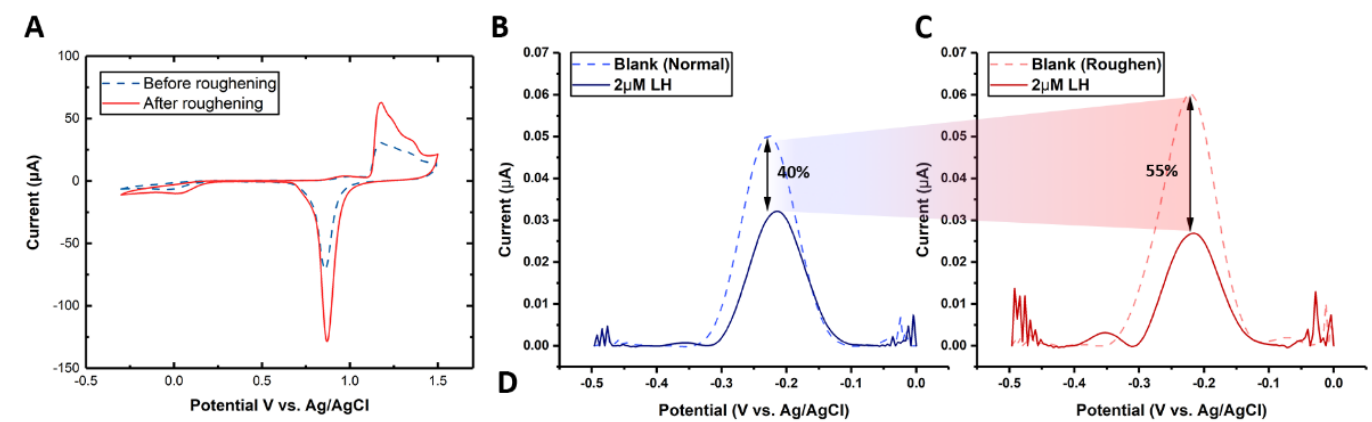

Figure 4-4: Methods for calculating the probe density. (A) CV scan of clean normal gold wire electrode in $0.5 \mathrm{M}$ sulfuric acid solution. The oxidation and reduction peaks increased after roughening. (B) Square-wave voltammogram of the response of normal wire electrode upon adding $2 \mu \mathrm{M}$ LH. (C) Square-wave voltammogram of the response of roughened wire electrode upon adding $2 \mu \mathrm{M} \mathrm{LH}$.

\subsection{Automatic system design}

\subsubsection{6 well plate platform design}

Based on the roughened wire electrode, I further developed an automatic system to perform the electrochemical measurement. Thanks to the convenient of the wire electrode, I succeeded to customise a 96 well plate format sensor. As shown in the Figure 4-5 A, I designed and 3D printed a plastic holder for the 3-wire-electrode system to adapt to a 96 well plate platform. The $3 \mathrm{D}$ printed adaptor and hold the 
wires separately to avoid electric shortcut, as well as leave enough space to allow tip movement for the automatic robot (Figure 4-5 B). Using this setup, the assay can be performed via moving the sample solution from the sample wells to the detection well. Wash step can be also integrated. Figure 4-5 C shows the picture of the system.
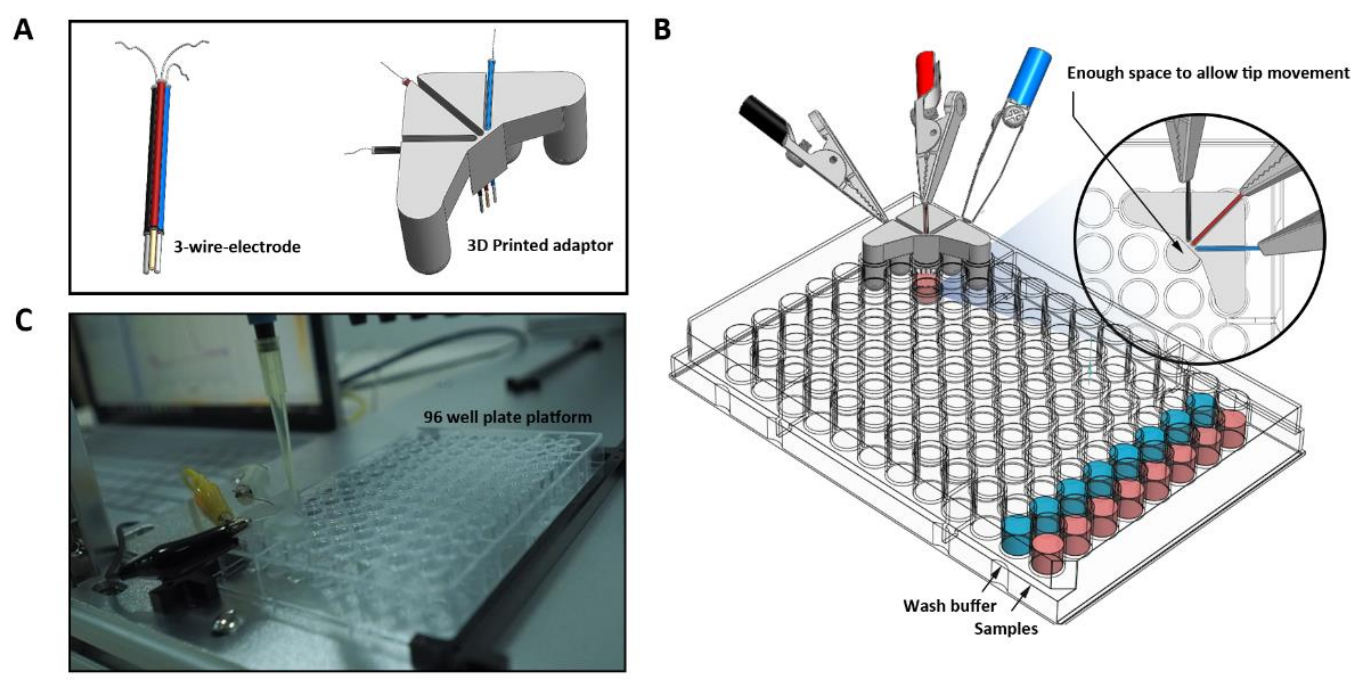

Figure 4-5: Design of the 96 well platform. (A) 3D printed adapter. The adaptor holds the 3 electrodes in a certain position to avoid electric shortcut (B) Assay format. Using 96 well plate the assay can be performed by moving sample solution from one well to another well and integrated with a wash step. (C) Picture of the platform.

\subsubsection{Automatic program}

I applied an open-source liquid handling robot for the automation of the electrochemical 96 well assay based on the wire electrode. The automatic program was written in Python under the opentron's API following a basic robot movement orders illustrated in Figure 4-6 A. First I need to calibrate the robot manually to allow the robot record every preset position of the 96 plate, tip box, tip discard box Figure 4-6 B. Then the program will be initiated and synchronize with the potentialstat auto measurement program by setting a time delay. The step loop is 1) the robot first moves the sample from the storage well to the detection well, wait until the measurement finish at the potentialstat; 2) move out the sample after measurement and discard the tip; 3) move the washing buffer and pipette up and down to regenerate the sensor, this includes discard the wash buffer and tips; 4) move the next sample and following the same loop Figure 4-6 C. 
A

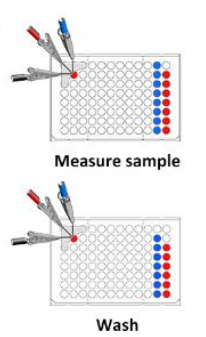

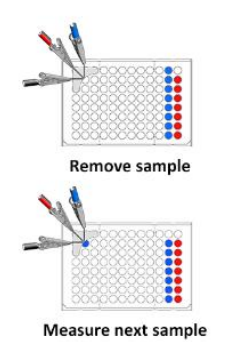

B

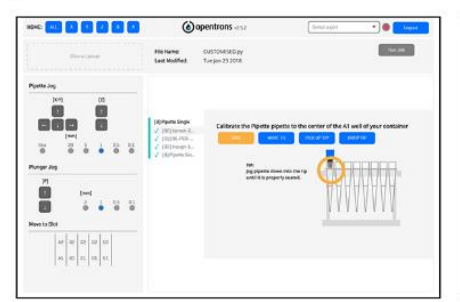

c

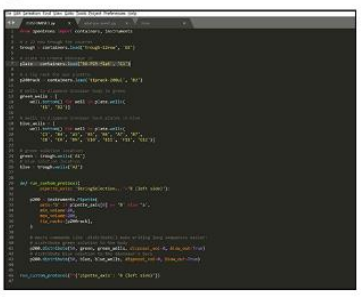

Figure 4-6: Design of the program. (A) Robotic movement orders (B) Calibration of the robot for recognising every preset position. (C) Python under Opentron's API.

\subsubsection{Assay performance}

An automatic assay was performed to evaluate the performance of the device (Figure 4-7 A). Base on the program designed, a series of different concentration LH solution were assayed continuously. The dose-response can be seen in Figure 4-7 B \& C. The roughened electrode has higher signal response range, the $K_{D}$ estimated was $259 \mathrm{nM}$, with a linear range from $5 \mathrm{nM}$ to $500 \mathrm{nM}$. FSH has also been used as the negative control.

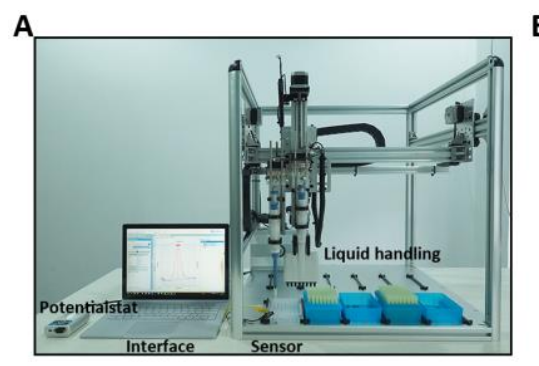

B

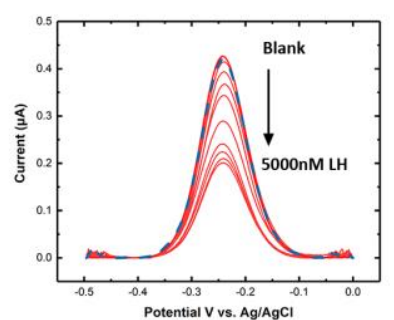

C

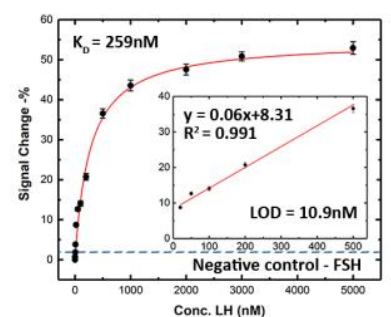

Figure 4-7: Assay performance in the system. (A) Automatic platform setup (B) SWV response, a current decreasing trend can be observed when increasing the LH concentration. (C) Dose-response curve for the LH titration study using the system.

\subsection{Attempts on designing other systems}

\subsubsection{Continuous flow system design}


I assembled a continuous flow electrochemical measuring platform using the wire electrodes and a plastic tube with a diameter of $1 \mathrm{~mm}$ (Figure 4-8 A). Three syringes with different concentration of LH in the PBS buffer (I also added 5\% HSA to simulate the serum condition) were switched subsequently after finishing the record of SWV signal under a certain time period. As can be seen from the Figure 4-8 B, there were clear responses every time I changed the concentration (pointed at the arrows in Figure 4-8 B). However, the baseline never returns to the origin. I assume the flow rate setting is not efficient enough for the sensor regeneration. In addition, I realised the baseline become more stable in the later concentration points. This may indicates the aptamer sensor requires a longer time for equilibration at the beginning.

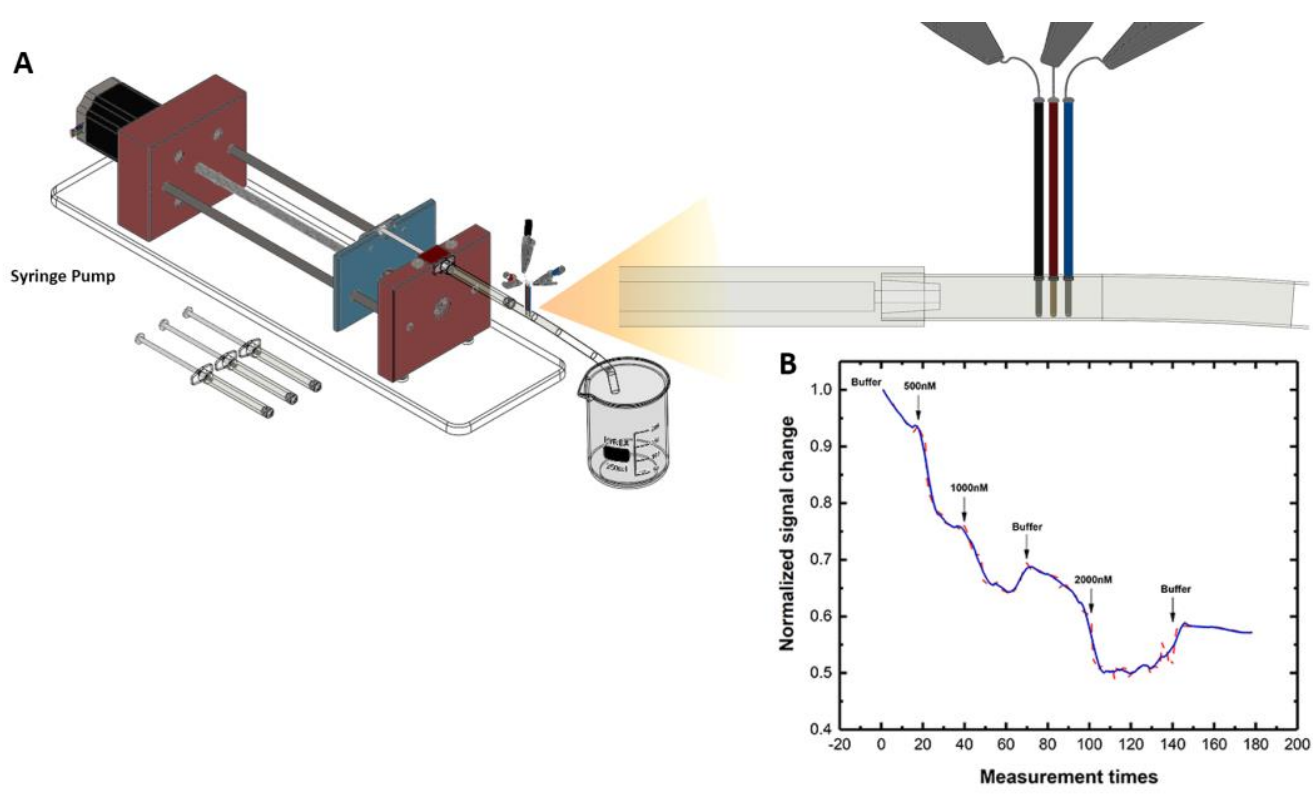

Figure 4-8: Continuous assay format. (A) Continuous device setup (B) Realtime response by injecting different concentration of LH in the device.

\subsubsection{Multiple-electrode chip based design}

Base on some of the positive results for the chemical deposited electrode, I designed multi-electrode system (48 individual electrode system per chip). Using this design, the LH pulsatility can be measured by direct dropping blood/serum sample onto the chip 48 times Figure 4-9 A. Unfortunately, I found out the electrode-electrode variation was considerable large even for the initial current signal (Figure 4-9 B) 
and I have to perform zero-point calibration before the usage of every single electrode so this design may need more improvement on the calibration.

A

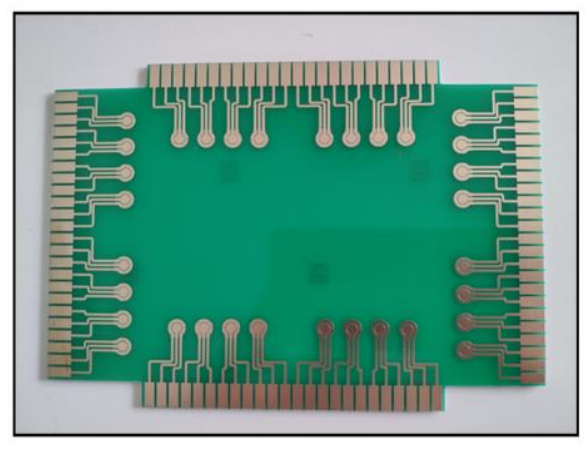

B

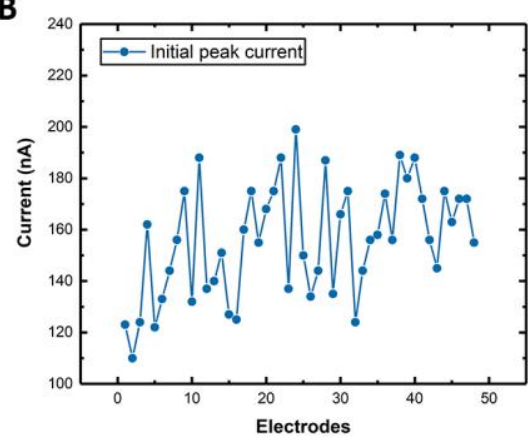

Figure 4-9: Multi-electrode chip format. (A) Design of the chip (B) Peak current in PBS for each electrode. This shows the variation of the peak currents.

\subsection{Chapter summary}

In this chapter, I rapid prototyped multiple devices based on the electrochemical LH aptamer assay developed previously. The first step towards customisation is to minimise the 3 -electrode system. The existing commercially available micro-chip electrodes showed unexpected results of no observing any aptamer signal (Figure 4-1). The cause of loss of signal was confirmed to be the failure of aptamer immobilisation on both Dropsens and Micrux electrode surfaces via the APTEC assay we developed before for another malaria aptamer. I don't have the answer yet why the immobilisation fails in these two electrodes as I found out the immobilisation was successful in other electrodes including chemical deposited gold, physically deposited gold electrode and traditional gold disc electrode (Figure 4-2). One possible reason for the failure of using Micrux electrode may be the working electrode area is too small ( $1 \mathrm{~mm}$ diameter) that no observable signal can be obtained.

Chemical and physical deposited gold electrodes are suitable to use as a disposable electrode format. However, I found out the physically deposited gold electrode I have has issued with peel-off gold. Therefore, I tried to design a 48 electrode array chip as one of the attempts I did for LH pulsatility measurement (Figure 4-9). Unfortunately, the initial signal readout varies from batch to batch 
and I failed to obtain consistent signal output without the zero-point calibration (perform a blank sample without LH and use the signal change to normalise signal output). This limits the application for the end user (too much to calibrate for 48 electrodes). Improvement may be added if I perform further optimisation on the electrode pre-treatment.

Therefore, I switch the focus to finding cheap alternatives for traditional gold disc electrode. Gold wire microelectrode was first used in 1994 for the measurement of Vitamin C in fruit juices [134]. Moreover, recently has been used in the E-AB field for continuous measurement [124]. I used 3D printing technology to generate different sensing format based on the gold wire electrode and characterised the wire electrode's performance using SWV (Figure 4-3). Electrochemical roughening increased the signal gain of the electrode, this is consistent with few other journals applied similar roughening method on the wire electrode $[121,123]$. I think the increased may occur due to the change of probe density, where provides larger degree of conformational change of aptamer upon binding to LH.

I further adapted opentron opensource liquid handling machine for our device prototype. The assay can be performed via moving and washing sample automatically under this system (Figure 4-5). The platform can be used for series clinical sample measurement and has the advantage of only using one sensor each time.

\subsection{Materials and Methods}

\subsubsection{Materials and equipment}

All chemicals and reagents were of analytical laboratory grade or higher. Screen printed electrode (Dropsens), Micrux electrode (MICRUX), APTEC reagents in details, all the modified aptamer used were synthesized in Biomer.net. The open source robot was purchased from Opentron. All the 3D printed parts were printed using Asiga 3D printer. All the metal wires were purchased from jewellery shop (Gold, silver, and platinum). Epoxy, soldering iron, soldering wire. Pump. 


\subsubsection{Sensor fabrication in commercialised electrodes}

A similar method was used to immobilise LH aptamer onto the screen-printed and micrux electrode. Prior immobilization, the electrodes were rinsed with absolute ethanol and $\mathrm{ddH}_{2} \mathrm{O}$. A drop of MB-B23 was added to each electrode for 4 hours incubation under a sealed moist condition. 200ul of $2 \mathrm{mM}$ Mercaptosuccinic acid (MSA) or $\mathrm{EG}_{3}$ or $\mathrm{MCH}$ was then used as a blocking reagent to block the unoccupied binding sites of the gold surface for 1 hour. The electrode was then saved in the binding buffer (PBS $+5 \mathrm{mM} \mathrm{MgCl}$ ) with parafilm sealed before the sensing measurement. Electrochemical measurement All of the measurements were performed with the system mentioned above.

\subsubsection{Sensor fabrication in customised electrodes}

The microneedle electrodes were kindly provided from Professor Tony Cass (Department of Chemistry, Imperial College London). A similar method for probe immobilisation was used for sensor fabrication. For the chemical deposit electrode, I used the same method for the probe immobilisation as the dropsens electrode.

\subsubsection{Fabrication of the gold wire electrode}

For each electrode, the gold wire was rinsed with ethanol and $\mathrm{ddH}_{2} \mathrm{O}$ to remove dust. Sandpaper (P320) was also used to polish the surface of the wires. The only $5 \mathrm{~mm}$ of the wire is exposed and electrodes were rinsed with absolute ethanol and $\mathrm{ddH}_{2} \mathrm{O}$. A drop of MB-B23 was added to each electrode for 4 hours incubation under a sealed moist condition. 200ul of 2mM Mercaptosuccinic acid (MSA) or $\mathrm{EG}_{3}$ or $\mathrm{MCH}$ was then used as a blocking reagent to block the unoccupied binding sites of the gold surface for 1 hour. The electrode was then saved in the binding buffer (PBS $+5 \mathrm{mM} \mathrm{MgCl}$ ) with parafilm sealed before the sensing measurement. Electrochemical measurement 


\subsubsection{Roughening of the electrode}

The roughening step was conducted by using two-step chronoamperometry by alternating the potential between $0 \mathrm{~V}$ to $2 \mathrm{~V}$, back and forth for around 10000 pulses in 0.5 Sulfuric acids.

\subsubsection{Opentron setup}

The opentron robotic platform needs to be calibrated before each time usage. The principle is to allow the robot to remember the preset position at each slot (such as 96 plates, tip box, trash etc.) by moving the liquid handling arm to the preset position manually. 


\section{CHAPTER 5 | Clinical evaluation of the automatic platform in patient samples with different LH pulsatility profiles}

In the previous 3 chapters, I demonstrated the development of an LH pulsatility sensing platform from generating the critical "binding molecule", optimising the suitable "electrochemical assay formats" to designing the integrated "device platform prototypes". While I can provide evidence that the system works in a solution-based system for LH detection, our ultimate goal is to apply the system from bench to bedside. In this chapter, I focus on evaluating our platform using actual clinical samples from patients with a different range of pulsatile LH profiles. These 650 samples are from 3 cohorts including menopause female, healthy male and female, as well as patients diagnosed Hypogonadotropic Hypogonadism. To further evaluate the clinical performance of our designed platform, I also performed comparison studies of our test results to the results obtained from the routine used clinical immunoassay system.

Keywords: menopause, hypogonadotropic hypogonadism, clinical assay, LH pulsatility

\subsection{Background}

Luteinising hormone pulsatility has been used as an indicator of hypothalamus function in many clinical studies, especially for studying the potential therapeutic agent that targets KNDy neuron level. In Dhillo's group, measuring LH pulsatility was used for studying NK3R antagonist for treating menopause hot flush [59, 135, 136], kisspeptin as a tool to assess GnRH function in primary amenorrhoea [136] and kisspeptin assisted ovulation induction [137, 138]. All of the LH pulsatility measurement in these studies were performed by clinical assay system which was costly and time-consuming as particular reagents and antibodies are required for each measurement. The development of the automatic E-AB platform provides an alternative option to measure LH in these samples and I can also use these clinical samples to exanimate our platform's performance further.

Therefore, to demonstrate, I chose clinical samples collected previously from 4 different cohorts: menopause [59], healthy male [139], healthy female [140], and 
HA sample collected alternatively under the ethic reference of $12 \mathrm{LO} / 0507$. All of the samples from these studies were from people who were recruited from newspaper advertisements and would come to our research unit and have at least one cannula inserted into a vein in their arm, which could then be used to repeatedly sample blood from. The sample for gonadotrophins and sex steroids were left to clot for at least 30 minutes, spun at 10,000 rpm for 10 minutes, the serum supernatant removed and then immediately stored at -20 for subsequent analysis using an automated chemiluminescent immunoassay method. Samples were taken from the vehicle or baseline visit. Each patient I used 48 samples and these samples were collected from the patient every 10 mins in 8 hours. I can apply one 96 plates for one patient's measurement (Figure 5-1).
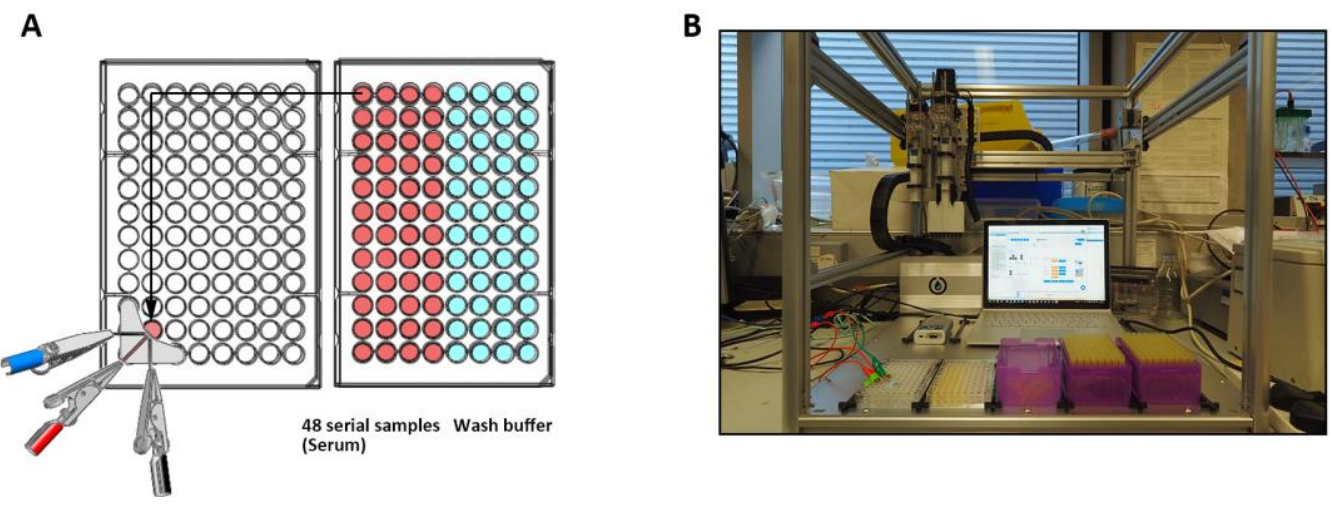

Figure 5-1: Methods for calculating the probe density. (A) CV scan of a clean gold electrode in $0.5 \mathrm{M}$ sulfuric acid solution (B) CV scan of an aptamer-immobilised electrode in PBS buffer

\subsection{Serum response}

To obtain a more accurate calibration curve for the sensing platform, I investigated the signal response change when I switch from normal binding buffer to serum condition. I directly used serum samples collected from patients with HA (Hammersmith Hospital, UK) and HH (Tsan Yuk Hospital, HK) as the blank reference. All of the serum samples were measured via Architect Plus system from Abbott and showed extremely low LH value (below the detection limit of the clinical assay system $=3 \mathrm{nM})$. As the LoD of our system in the solution was estimated to be $10.9 \mathrm{nM}$, I believed those sample could be used as the blank reference as their clinical values are less than our detection limit. Here I used these samples to investigate the sensor's signal response and regeneration performance. 


\subsubsection{Conversion of the molar concentration to the international unit}

In the normal clinical practice, LH concentration is measured in international units (IU). Therefore in the clinical evaluation studies, all the results generated from our sensing platform were converted to IU based on the 96/602 standard reference (same standard as the LH I used for calibration). In this standard, one IU is defined as $1 / 189^{\text {th }}$ of an ampule donated $96 / 602$ and distributed by the NIBSC. It is equal to $0.04656 \mu \mathrm{g}$ of $\mathrm{LH}$. Therefore for $1 \mathrm{IU} / \mathrm{L}$, it is approximately $5.6 \mathrm{nM}$.

\subsubsection{Electrochemical signal response in undiluted serum}

5 samples from 5 HA patients with LH level under $0.1 \mathrm{IU} / \mathrm{L}$ (approximately $0.56 \mathrm{nM}$, measured by Architect Plus system) were mixed and used as the blank reference for this study. I performed both CV and SWV after I move the undiluted serum sample to the sensing area of the device. In the Cyclic Voltammogram shown in Figure 52 A, I observed a potential shift from $-0.24 \mathrm{~V}$ to $-0.28 \mathrm{~V}$, and the $\mathrm{H}_{2} \mathrm{O}$ oxidation became weaker (the negative "tail" of the blue curve became shorter in the red curve). This may suggest the absorption of large proteins in the serum onto the electrode surface. However, in the Square-wave voltammogram presented in Figure 5-2 B, there was no obvious signal reduction, but a significant peak shift correspond to the $\mathrm{CV}$ study from $-0.24 \mathrm{~V}$ to $-0.28 \mathrm{~V}$. This may suggest the nonspecific absorption do not change the aptamer structure. I then performed a titration study using the same concentration range of the dose-response test of spiked LH in PBS buffer described in the previous chapter but this time with spiked LH in undiluted HA serum sample. As can be seen in the Figure 5-2 C, the $\mathrm{K}_{\mathrm{D}}$ estimated is $358 \mathrm{nM}$, a bit higher than the dose-response study under PBS buffer condition $(259 \mathrm{nM})$. The LoD measured was similar with a value of $10.7 \mathrm{nM}$, which approximately equals $1.9 \mathrm{IU} / \mathrm{L}$. 

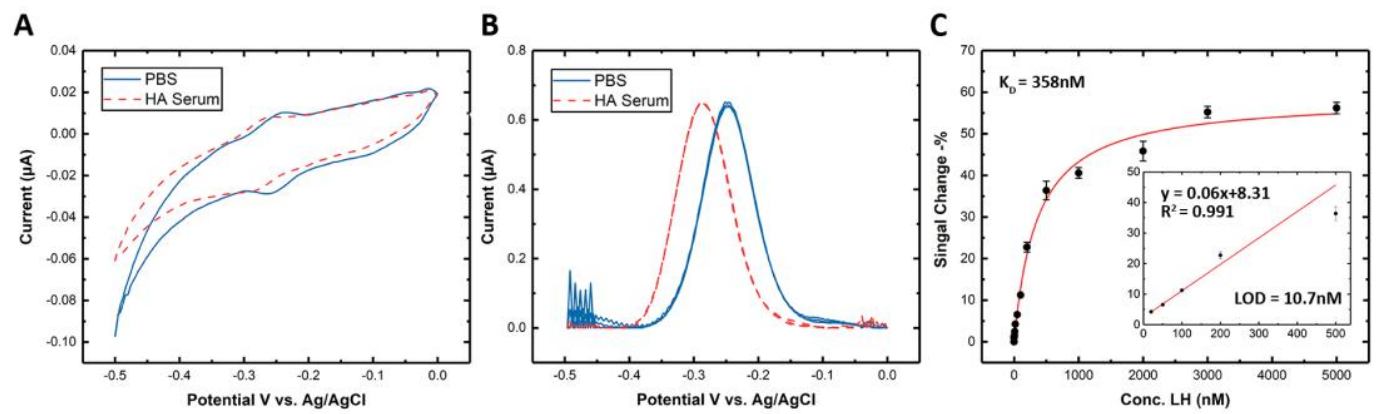

Figure 5-2: Dose-response of the sensing system in undiluted serum. (A) CV scan of the LH aptamer sensor in PBS and then in undiluted HA serum. (B) SWV of the LH aptamer sensor in PBS and then in undiluted HA serum. (C) Doseresponse curve using SWV methods. The "-\%" of the signal change indicates the change is a "signal off" model. The $\mathrm{K}_{\mathrm{D}}$ is estimated to be $3580 \mathrm{nM}$, and the linear range is from $10 \mathrm{nM}$ to $500 \mathrm{nM}$.

\subsubsection{Regeneration in a serum sample}

I further investigated the signal regeneration of the sensing platform under HA serum condition using SWV. According to the previous SPR result, the off rate of the B23 aptamer is relatively fast (with a half-life of about 12 minutes). I want to see under normal wash condition, whether the sensor can be regenerated. Figure 53 shows the Square-wave voltammogram of the regeneration study. I first applied $500 \mathrm{nM}$ of $\mathrm{LH}$, which is about $2 \mathrm{x}$ higher than the maximum $\mathrm{LH}$ concentration I will be using in the clinical investigation $(50 \mathrm{IU} / \mathrm{L})$. By normal washing step performed by the robotic platform, the signal can be regenerated in a relatively completed level (94\%). However, when 5000nM of LH applied, only can be regenerated to $75 \%$. I think this may not affect much as our clinical application range for the pulsatility measurement normally under 500nM. 


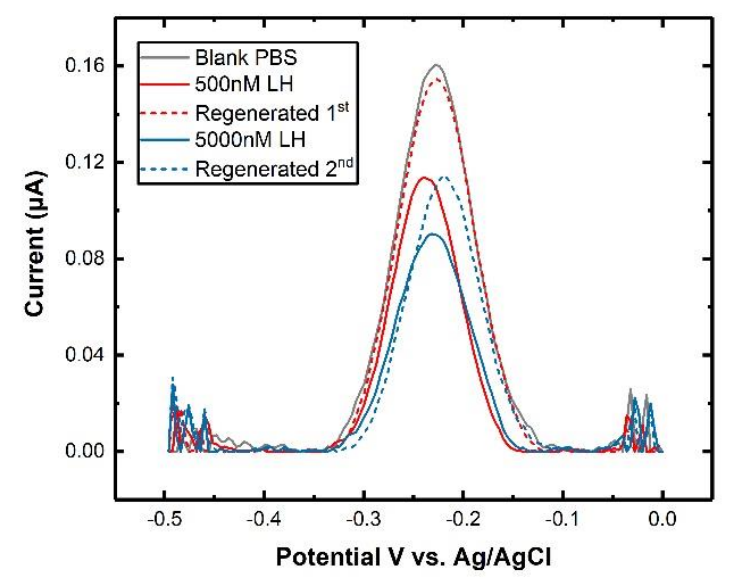

Figure 5-3: Regeneration under undiluted serum condition. The dash line represents the regenerated peak obtained after using the automatic platform washing step

\subsection{Small-scale test}

Before I use the system to perform large-scale study, I performed two small-scale studies using serum samples collected from two regions (the UK and Hong Kong) and compared the result from our sensing platform to the results obtained from the clinical assay system. The first pilot study is a measurement of 6 individual serum samples from a healthy female, $\mathrm{LH}$ concentration of these ranging from 3.7IU/L $(20.72 \mathrm{nM})$ to $28.7 \mathrm{IU} / \mathrm{L}(160.72 \mathrm{nM})$. The mixture of HA serum sample was used as the blank reference to calibrate the device. The final calculation of the LH concentration via our sensing system utilised the Hyperbl fitting equation from Figure 5-2 C and converted to IU/L from nM. As can be seen in the Figure 5-4 A, the result from our sensing platform is relevant to the result obtained from the clinical assay system. A Bland Altman plot was used to compare two methods, mean value is closed to 0 which this two assays results are relevant.

A second pilot study was performed using human serum samples with selected concentrations to stimulate three pulses. Figure 5-4 C and D show the comparison of the measured result to the clinical assay result. They all show a consistent concentration range. 
A

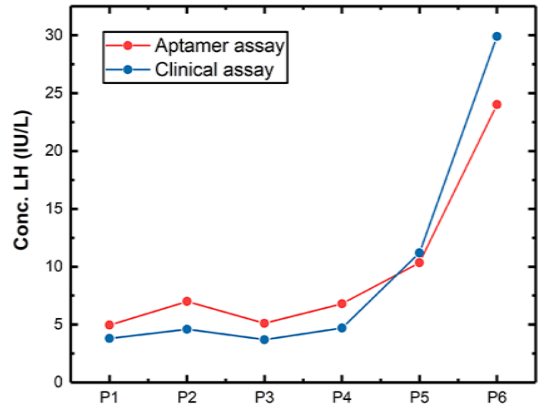

Clinical samples with different $\mathrm{LH}$ concentrations

C

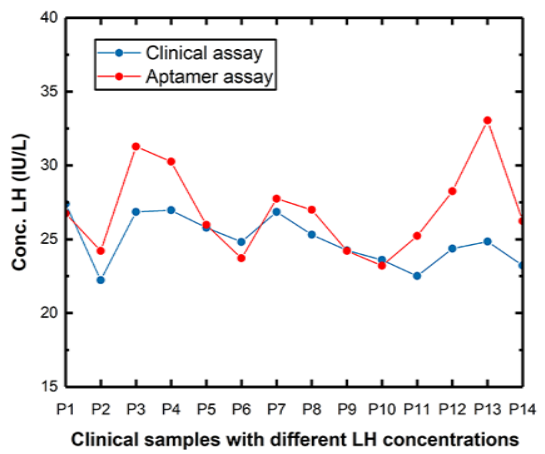

B

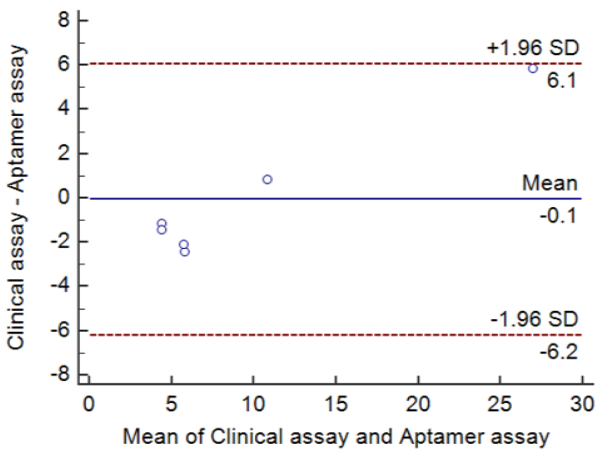

D

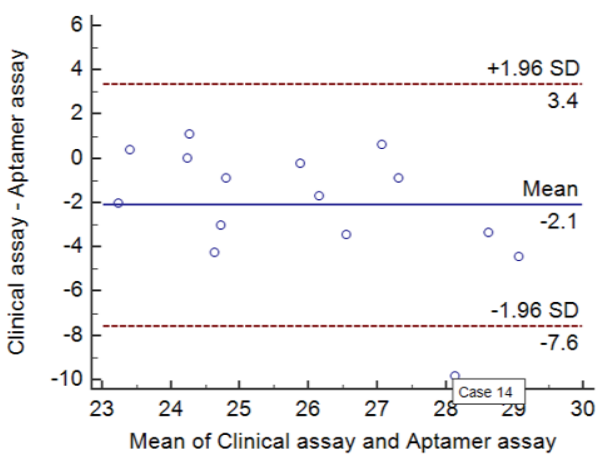

Figure 5-4: Pilot studies on measuring LH pulsatility. (A \& B) 6 patients with different large range LH level measured by the aptamer and clinical assay and the Bland Altman plot (C \& D) 14 patients with small range variation LH level measured by the aptamer and clinical assay and the Bland Altman plot.

\subsection{Menopause patient samples (high LH level)}

A larger scale of study using 150 samples collected from menopause female was conducted to further evaluate the platform's performance in relatively high LH and FSH level sample. Menopause female usually has more frequent LH pulse and high LH amplitude. The FSH level is much higher than the LH level in those selected samples. I chose 3 patient's profile where their $\mathrm{LH}$ level is ranging from $14.15 \mathrm{IU} / \mathrm{L}$ $(79.24 \mathrm{nM})$ to $49.89 \mathrm{IU} / \mathrm{L}(279.38 \mathrm{nM})$. There were in total 150 samples and each of the patients was measured using hand fabricated wire electrodes $(n=3)$. Figure 5-5 A to $\mathbf{C}$ shows the concentration match for the clinical assay and Aptamer assay, Figure 5-5 E to $\mathbf{G}$ show the difference between FSH value and the measured LH value from our sensing platform. All of the obtained datapoints were plotted using Bland Altman method to compare the relativity of two measuring methods 
(Aptamer and antibody). As can be seen in the Figure 5-5 D, the plot's mean value is very close to zero.
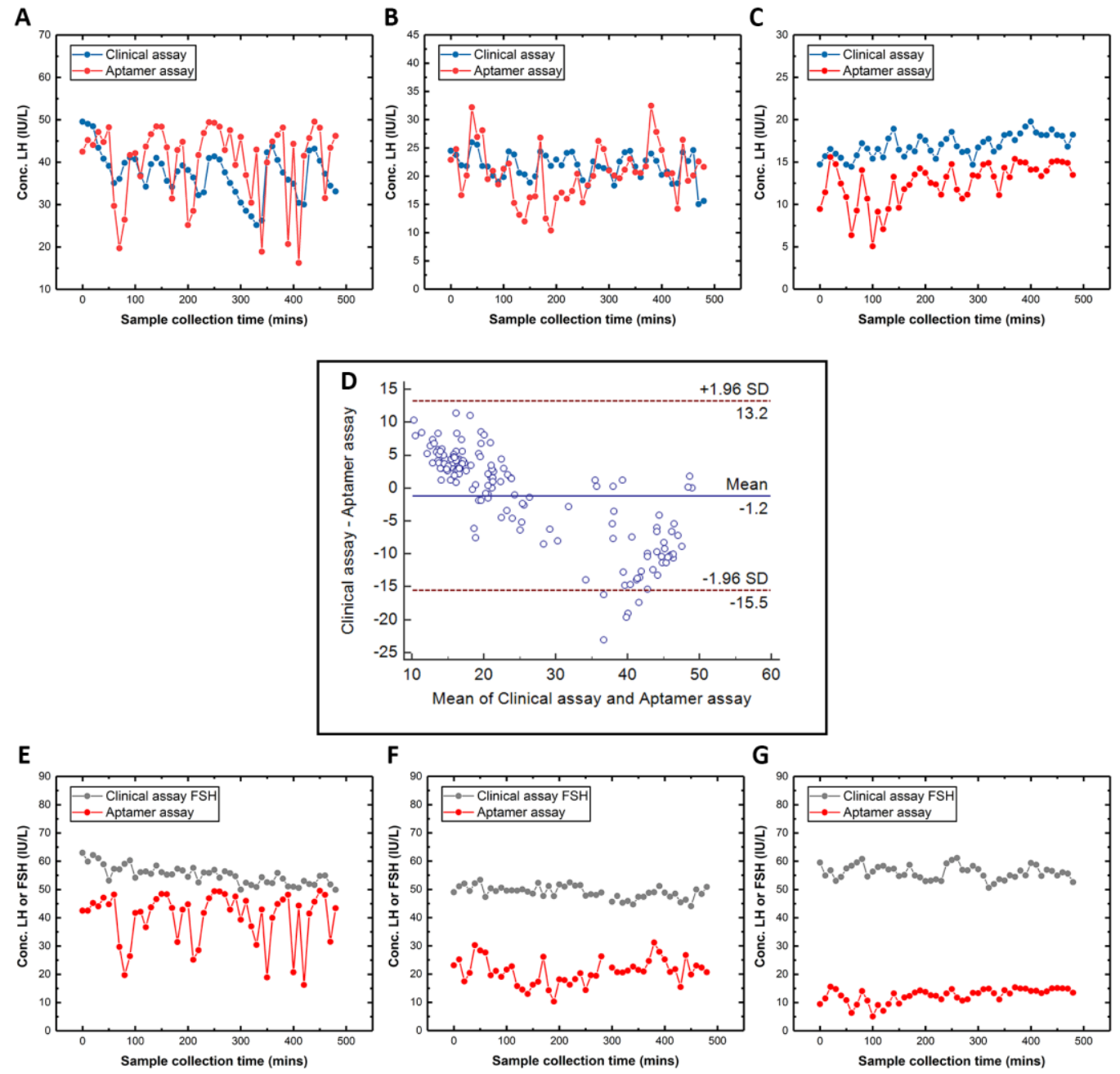

Figure 5-5: Analysis of the LH pulsatility measurement of menopause female samples.

(A B C) 3 patients with different range LH level measured by the aptamer and clinical assay (D) Bland-Altman plot (E F G) 3 patients with different range FSH level measured by the clinical assay and LH level measured by the aptamer assay 


\subsection{Health people samples (normal LH level)}

\subsubsection{Serum samples collected from healthy female}

The other cohort I want to measure is the healthy male and female group. The project aims to distinguish between health and patient with abnormal LH pulsatility, such as HA patient. It is therefore more important to see what is the actual performance of the system when it is used for healthy cohorts. I first collected serum samples from 2 sub-groups (female and male) and their LH value measured by the clinical assay system is ranging from 2-5IU/L (male) and 2-8 (female). As shown in the Figure 5-6 A, B, and C, 3 patients with 150 serum samples were measured using the aptamer system and compared the results with clinical assayed value. In the female group, the result still shows a very relevant line even though one of them exhibit the calculated range is not at the same level of the clinical assay (Figure 55 B), this may be due to some of the LH degradation. Bland-Altman plot was again used to determine the relativity of these two assays (Figure 5-6 D).
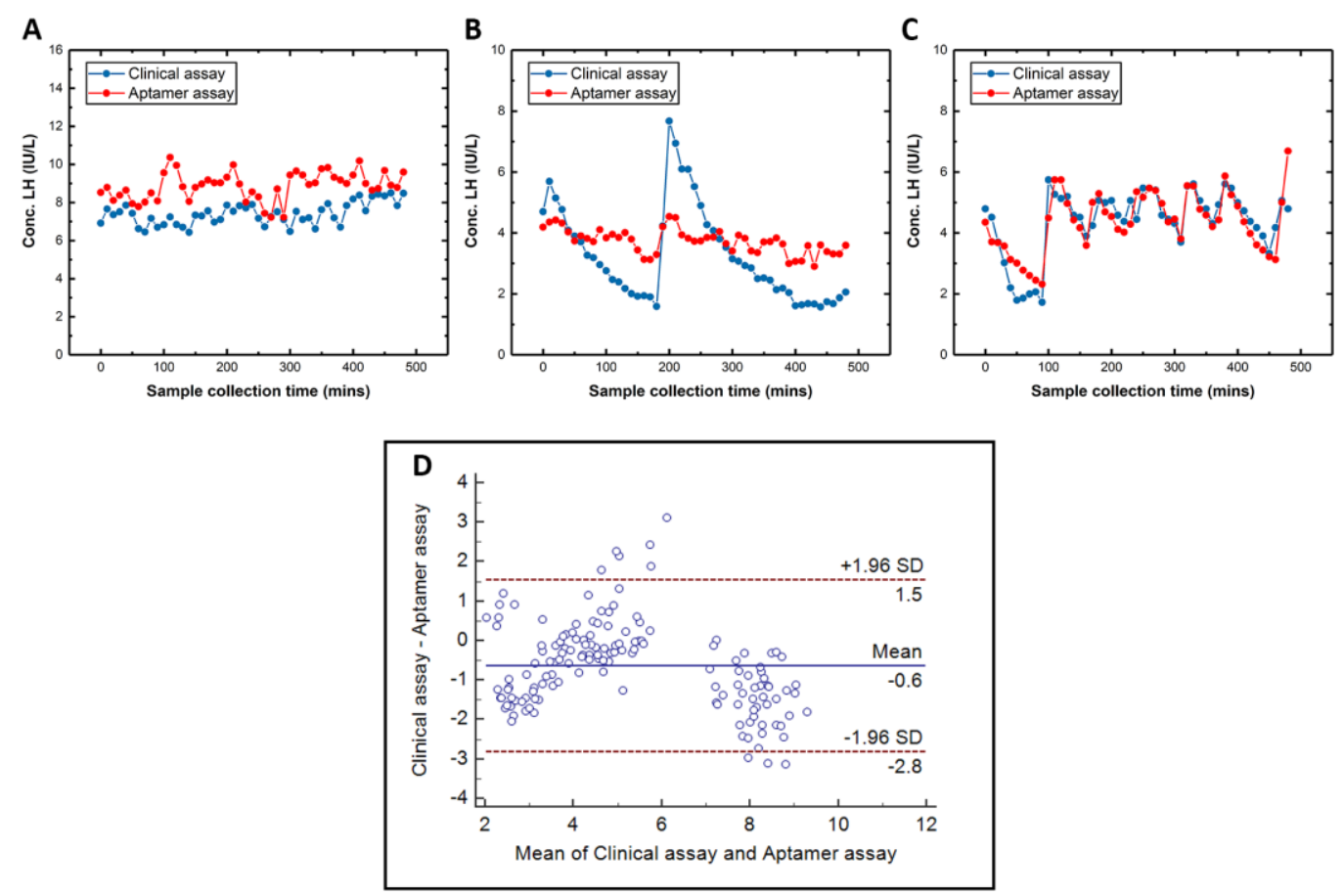

Figure 5-6: Analysis of the LH pulsatility measurement of healthy female samples. (A B C) 3 patients with different range LH level measured by the aptamer 
and clinical assay (D) Bland-Altman plot comparing the clinical assay result and clinical assay result in the whole healthy female patient group samples

\subsubsection{Serum sample collected from healthy male}

However, in another study of using the male serum sample for the LH pulsatility measurement, I didn't observe the optimal response of the LH binding aptamer. Figure 5-7 A-C shows the comparison between clinical assay and aptamer assay, from there I observed signal drift especially in the Figure 5-7 B, I suggest this may due to the concentration in these sample sets are all quite close to the detection limit of the system (around 2IU/L), low signal to noise ratio at this range makes the measurement more difficult. Figure 5-7 D shows the Bland-Altman plot for the two methods. The mean value is 0.7 which is till quite close to the 0 point.
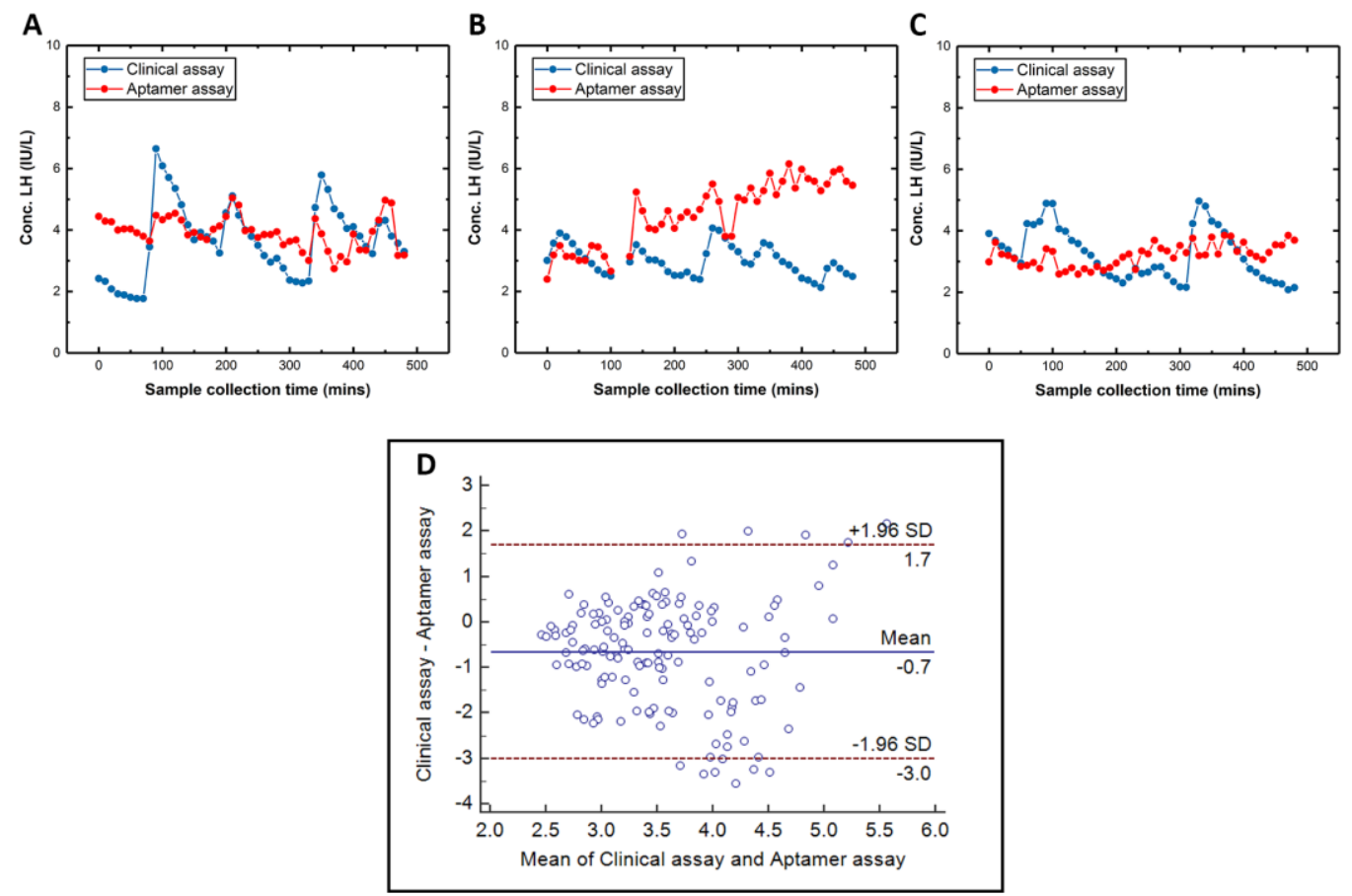

Figure 5-7: Analysis of the LH pulsatility measurement of healthy male samples. (A B C) 3 patients with different range LH level measured by the aptamer and clinical assay (D) Bland-Altman plot comparing the clinical assay result and clinical assay result in the whole healthy male patient group samples 


\subsection{HA samples (no LH)}

Finally, I performed a continuous measurement of our blank reference sample - the serum located from HA patient. In total 146 samples were used in our platform and the processed results are list below in Figure 5-8 A, B, and C). None of them can be well quantified as they are below the detection limit.
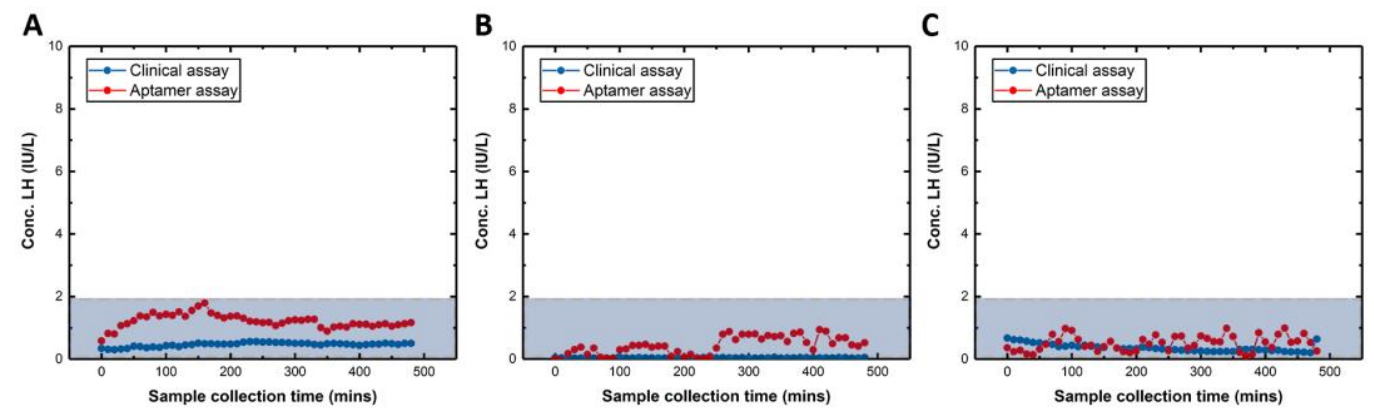

Figure 5-8: (A B C) Three HA patients pulsatility profile. All the data points were below the detection limit of the platform,

\subsection{Chapter summary}

In this chapter, I performed the clinical evaluation of our automatic LH aptamer assay system. I chose clinical samples with a different range of LH level as I want to find out whether the device works to distinguish among different patient cohorts. I also used clinical samples from patients in different regions (6 patients from Hong Kong and 12 from UK). Using the HA patient sample, I found out the blank serum didn't affect the sensor performance very much (Figure 5-2). I also test the regeneration of the sensor and found out $95 \%$ regeneration can be achieved within the linear range of the sensor (Figure 5-3). These results, together with the successful pilot studies (Figure 5-4) gave us the confidence to carry out the larger trial for LH pulsatility measurement.

Menopause samples gave us the best pulse match which is expectable as the serum level of LH falls into the high concentration range within the linear curve. All of this samples were measured both LH and FSH level using the clinical immunoassay, and there is a clear difference between the FSH level and the LH 
level I measured using our device. This is an evidence of the capability our LH sensor to distinguish FSH and LH in a serum sample (Figure 5-5).

Similar results can be observed in our health female sample, in 3 of the health objects, the LH measurement using our device platform fits well for 2 healthy female, with 1 exception (Figure 5-6). II did notice in this patient sample, the value I obtained of the LH is quite close to the FSH value. In the health male sample, especially when II measured low LH level's sample. The device can rarely pick up the pulse which is understandable as the LH level of the sample I have is quite close to the detection limit of our platform (Figure 5-7). Therefore I think I still need to improve the signal-to-noise ratio for the final application in healthy people.

However, I could positively distinguish the HA patients' sample to the healthy and menopause patients' sample as there is no LH presented in the HA sample (Figure 5-8).

\subsection{Materials and Methods}

\subsubsection{Material and equipment}

The 6 healthy woman serum samples for the $1^{\text {st }}$ pilot study and the HA patient serums were kindly offered by Dr. Raymond Li from Tsan Yuk Hospital, HK. The rest of the samples were collected at the Hammersmith Hospital, UK. They were ollected alternatively under the ethic reference of 12LO/0507. All of the samples from these studies were from people who were recruited from newspaper advertisements and would come to our research unit and have at least one cannula inserted into a vein in their arm, which could then be used to repeatedly sample blood from. The sample for gonadotrophins and sex steroids were left to clot for at least 30 minutes, spun at 10,000rpm for 10 minutes, the serum supernatant removed and then immediately stored at -20 for subsequent analysis using an automated chemiluminescent immunoassay method. Samples were taken from the vehicle or baseline visit. Each patient I used 48 samples and these samples were collected from the patient every 10 mins in 8 hours. The equipment used for clinical assay measurement was Architect Plus system from Abbott. 


\subsubsection{Automatic assay procedure}

The OpenTron robot was calibrated before each patient measurement. $10 \mathrm{HA}$ patient serum samples with undetectable LH concentration were poured together and 0 point calibration was performed for each electrode. The measurement cycle was: 1 ) move sample $>2$ ) measure sample $>3$ ) remove sample/wash electrode $>4$ ) move next sample. The SWV measurement was initiated at the Emstat interface (PSTrace) with a set time delay to synchronise with the robot. All the square-wave voltammograms obtained were normalised in PSTrace and recorded the peak value of each sample. The data of the peak value was processed and analysis via Excel, Origin and MedCal. 


\section{CHAPTER 6 | Perspectives and outlook}

\subsection{Summary}

Back to Geoffrey Harris's age in 1955, the evidence obtained to support his HPG axis hypothesis was mainly from experiments done in female since ovulation was the only reliable surrogate marker for hypothalamic activation to be detected [141]. Nowadays, thanks to the development of hormone assay methods, I am able to choose more accurate maker to investigate HPG axis function. LH pulsatility has been widely used in the academic research as the optimal surrogate marker for GnRH pulsatility [31] for investigating hypothalamic function both in animal models [142, 143] and human clinical studies [49, 59]. However, limitation remains on its clinical usage as the current immunoassay system is not designed for pulsatility measurement $[29,30]$. Therefore, to address this, I developed an entire novel device platform from the very basic binding molecule and evaluated the performance via conducting measurement in real clinical samples with different LH pulsatility profile.

I have 3 significant novelty in this research project:

1) Novel aptamer against LH with high affinity and specificity. Current no reported LH aptamer exist, and I successfully adapted this newly developed aptamer into electrochemical assay format. (Chapter $2 \&$ Chapter 3)

2) The automation of $E-A B$ sensing. The automation of $E-A B$ sensing provides a competitive platform to the current clinical immunoassay platform with a smaller footprint, lower cost and faster assay time. (Chapter 4)

3) Evaluation of the platform via multiple patient cohorts and obtain a comparison result to the clinical assay. The pilot studies I performed provide a proof-ofconcept in applying the E-AB sensing system in the clinic. (Chapter 5)

\subsection{Perspectives}


In chapter 2, I presented the development and characterisation of our novel LH aptamer. Traditional SELEX has been widely used in our lab, and I have developed multiple aptamers based on these methods [96-99]. The reason I choose nitrocellulose membrane selection is I don't have any protein tag in our recombinant human LH protein for the selection. There is currently no LH aptamer exist in the literature, only hCG peptide aptamer [144] and Ovine FSH $\alpha$ subunit DNA aptamer [145] have been reported. As the fact that our LH DNA aptamer is specific to LH but not FSH so I assumed the binding side of the aptamer might be in the $\beta$ subunit which gives us more novelty of the project.

The optimal LH aptamer candidate should meet the three requirements: First, strong binding affinity against LH and no significant interference with LH structural similar proteins such as FSH and TSH; Second, upon binding the LH, the candidate should trigger a certain degree of conformational change so that it can be further modified based on the structure-switching electrochemical sensing scheme; And the third, the candidate would preferably have a relatively fast off-rate so that the sensing response can be recorded in a real-time format. I applied biochemical and biophysical assay to characterise our aptamer candidates, and the result shows the LH specific aptamer could meet three of these requirements. However, judging from later result of the continuous format sensing platform, I may think the current off-rate of the aptamer I have is not sufficient for quick regeneration similar to most of the small molecule aptamer sensors exist in the literature [110, 124]. Normal small molecule aptamer has dissociation rate around $10^{-2}$ to $10^{-3} \mathrm{~s}^{-1}[146,147]$ whereas our LH aptamer is $1.2 \times 10^{-3} \mathrm{~s}^{-1}$, which encountered difficulty when applied it in a complex sample such as serum and blood. This is one of the reasons in the later I switched toward using the strategy of designing multiple measurement device systems and I hope to improve the continuous sensing ability of this aptamer in the future.

In chapter 3 and chapter 4 , I present the development of the electrochemical assay based on our LH aptamer under a traditional gold disk electrode platform and the minimisation of this assay into a smaller microwire electrode platform. Polycrystalline gold has long been commonly used as the working electrode in the typical three-electrode electrochemical sensing system [148, 149]. The robust feature of this type of gold disc electrode and can be easily reused made us choose it as a standard model for studying the electrochemical properties of the redox probe labelled LH aptamer. By applying this system, I investigated the use of different 
redox probes, packing density of the aptamer on the defined electrode surface, as well as the co-thiol molecule selection.

One of the most controversial conclusions I have during the optimisation process is the usage of Fc as the redox probe. Fc has been widely used in the electrochemical aptamer-based sensor design [150, 151]. However, I found out using $\mathrm{Fc}$ as the redox probe for reuse type of sensing may be problematic. As can be seen in our result (Figure 3-3), the baseline keeps decreasing when I performed repeated measurements in the same sample. One possibility is the ferrocenium formed may prone to nucleophilic attack and the Fc molecule is then be taken off from the nucleic acid [152]. Also, when I performed studies using commercially available chip-based electrodes, unknown peaks in the potential close to where Ferrocene is. The minimisation of the sensor was achieved using microwire electrode. Gold wire microelectrode was first used in 1994 for the measurement of Vitamin C in fruit juices [134]. Moreover, recently has been used in the E-AB field for continuous measurement [124]. I adapted the design of this microwire into the 96 well plate assay system and achieved the automation under the opensource robots.

In chapter 5, I performed the clinical evaluation of our automatic LH aptamer assay system. I choose clinical samples with a different range of LH level as I think I want to find out whether the device works to distinguish among different patient cohorts. In Dhillo's group, measuring LH pulsatility has been used for investigating NK3R antagonist for the treatment of menopause hot flush [59, 135, 136], kisspeptin as a tool to assess GnRH function in primary amenorrhoea [136] and kisspeptin assisted ovulation induction [137, 138]. All of the LH pulsatility measurement in these studies were performed by clinical assay system which was costly and time-consuming as particular reagents and antibodies are required for each measurement.

Therefore, our LH pulsatility measuring system has the advantage regarding less sample volume requirement and only one-step required for generating signal out. The LH pulsatility profile obtained from our system were similar to what the result obtained from the clinical system, which gives us more confidence on the further development to improve this LH pulsatility sensing platform in the future. 


\subsection{Future work}

To revisit our motivation on using aptamer technology for LH pulsatility measurement, I realise that I have not developed a continuous sensing platform that proved in the clinic but rather than that, I developed a proof-of-concept platform that close to this goal and proved it has its competitivity to the current assay system. The AELECAP system can also be further improved for multiplex measurement, a panel of aptamers can be immobilised onto the microwires and integrated into the platform using a newly designed 3D printed holders. These multiplex AELECAP system can be applied to multiple hormone pulsatility monitoring including cortisol and growth factor. A better software interface that can synchronise OpenTron program and potentialstat program is also needed. To fulfil the clinical usage requirement, an integration of automatic blood sampling device in the liquid handling robot is a good direction.

In the future, I also hope to further optimise the continuous platform design (Figure 4-8) with better signal to noise control and drift correction process. To do this, further optimisation or a new generation of LH aptamer is needed. The critical properties of the optimal continuous sensing aptamer are 1) wide "signal on" range; 2) fast dissociation rate; Unfortunately there is currently no selection method that is targetting these requirements. For the second point, the application of SPR in the selection may help as SPR can offer real-time binding kinetic information during the library selection.

How to better process the data is also required further investigation. I have not yet used pulsatility program to fit the data. Deconvolution model as mentioned in the first chapter can be used to analyse the pulse information for both the LH pulsatility result measured by AELECAP and original clinical immunoassay platform. Therefore, more mathematical modelling needs to be done in the future for data interpretation.

I aspire to disseminate the LH aptamer platform for usage and further development. Ultimately, I hope that this work and future directions will help bring hormone pulsatility measurement closer to reaching its potential clinical application. 


\section{Reference}

1. Todd RB. The Cyclopaedia of Anatomy and Physiology. 1836:460.

2. Tata JR. One hundred years of hormones - A new name sparked multidisciplinary research in endocrinology, which shed light on chemical communication in multicellular organisms. Embo Rep 2005; 6: 490-6.

3. Gay VL, Midgley AR, Jr. Response of the adult rat to orchidectomy and ovariectomy as determined by LH radioimmunoassay. Endocrinology 1969; 84: 1359-64.

4. Veldhuis JD, Keenan DM, Pincus SM. Motivations and methods for analyzing pulsatile hormone secretion. Endocrine reviews 2008; 29: 823-64.

5. Krsmanovic LZ, Stojilkovic SS, Catt KJ. Pulsatile gonadotropin-releasing hormone release and its regulation. Trends in endocrinology and metabolism: TEM 1996; 7: 56-9.

6. Vance ML, Kaiser DL, Evans WS et al. Pulsatile growth hormone secretion in normal man during a continuous 24-hour infusion of human growth hormone releasing factor (1-40). Evidence for intermittent somatostatin secretion. The Journal of clinical investigation 1985; 75: 1584-90.

7. Scheff JD, Kosmides AK, Calvano SE et al. Pulsatile glucocorticoid secretion: origins and downstream effects. IEEE transactions on bio-medical engineering 2011; 58: 3504-7.

8. Pitteloud N, Dwyer AA, DeCruz S et al. The relative role of gonadal sex steroids and gonadotropin-releasing hormone pulse frequency in the regulation of folliclestimulating hormone secretion in men. The Journal of clinical endocrinology and metabolism 2008; 93: 2686-92.

9. Levine JE, Chappell P, Besecke LM et al. Amplitude and frequency modulation of pulsatile luteinizing hormone-releasing hormone release. Cellular and molecular neurobiology 1995; 15: 117-39.

10. Fraietta R, Zylberstejn DS, Esteves SC. Hypogonadotropic hypogonadism revisited. Clinics 2013; 68 Suppl 1: 81-8.

11. Hartman ML, Iranmanesh A, Thorner MO, Veldhuis JD. Evaluation of pulsatile patterns of growth hormone release in humans: A brief review. American journal of human biology: the official journal of the Human Biology Council 1993; 5: 60314.

12. Keenan DM, Veldhuis JD. Pulsatility of Hypothalamo-Pituitary Hormones: A Challenge in Quantification. Physiology (Bethesda) 2016; 31: 34-50.

13. Fluhmann CF. Anterior pituitary hormone in the blood of women with ovarian deficiency. JAMA 1929; 93: 672-4.

14. Harris GW. Neural control of the pituitary gland. LONDON: Edward Arnold 1955.

15. Amoss M, Burgus R, Blackwell $R$ et al. Purification, amino acid composition and $\mathrm{N}$-terminus of the hypothalamic luteinizing hormone releasing factor (LRF) of ovine origin. Biochem Biophys Res Commun 1971; 44: 205-10. 
16. Matsuo H, Baba Y, Nair RM et al. Structure of the porcine LH- and FSH-releasing hormone. I. The proposed amino acid sequence. Biochem Biophys Res Commun 1971; 43: 1334-9.

17. Dierschke DJ, Bhattacharya AN, Atkinson LE, Knobil E. Circhoral oscillations of plasma LH levels in the ovariectomized rhesus monkey. Endocrinology 1970; 87: 850-3.

18. Belchetz PE, Plant TM, Nakai Y et al. Hypophysial responses to continuous and intermittent delivery of hypopthalamic gonadotropin-releasing hormone. Science 1978; 202: 631-3.

19. Clarke IJ, Cummins JT. The temporal relationship between gonadotropin releasing hormone $(\mathrm{GnRH})$ and luteinizing hormone $(\mathrm{LH})$ secretion in ovariectomized ewes. Endocrinology 1982; 111: 1737-9.

20. Dalkin AC, Haisenleder DJ, Ortolano GA et al. The frequency of gonadotropinreleasing-hormone stimulation differentially regulates gonadotropin subunit messenger ribonucleic acid expression. Endocrinology 1989; 125: 917-24.

21. Tsutsumi R, Webster NJ. GnRH pulsatility, the pituitary response and reproductive dysfunction. Endocr J 2009; 56: 729-37.

22. de Roux N, Genin E, Carel JC et al. Hypogonadotropic hypogonadism due to loss of function of the KiSS1-derived peptide receptor GPR54. Proc Natl Acad Sci US A 2003; 100: 10972-6.

23. Seminara SB, Messager S, Chatzidaki EE et al. The GPR54 gene as a regulator of puberty. N Engl J Med 2003; 349: 1614-27.

24. Topaloglu AK, Reimann F, Guclu M et al. TAC3 and TACR3 mutations in familial hypogonadotropic hypogonadism reveal a key role for Neurokinin B in the central control of reproduction. Nat Genet 2009; 41: 354-8.

25. Ahn T, Fergani C, Coolen LM et al. Prenatal testosterone excess decreases neurokinin 3 receptor immunoreactivity within the arcuate nucleus KNDy cell population. $J$ Neuroendocrinol 2015; 27: 100-10.

26. Lehman MN, Coolen LM, Goodman RL. Minireview: kisspeptin/neurokinin B/dynorphin (KNDy) cells of the arcuate nucleus: a central node in the control of gonadotropin-releasing hormone secretion. Endocrinology 2010; 151: 3479-89.

27. Li XF, Kinsey-Jones JS, Cheng Y et al. Kisspeptin signalling in the hypothalamic arcuate nucleus regulates GnRH pulse generator frequency in the rat. PloS one 2009; 4: e8334.

28. Knobil E. The GnRH pulse generator. American journal of obstetrics and gynecology 1990; 163: 1721-7.

29. Sauder SE, Frager M, Case GD et al. Abnormal patterns of pulsatile luteinizing hormone secretion in women with hyperprolactinemia and amenorrhea: responses to bromocriptine. J Clin Endocrinol Metab 1984; 59: 941-8.

30. Reame NE, Sauder SE, Case GD et al. Pulsatile gonadotropin secretion in women with hypothalamic amenorrhea: evidence that reduced frequency of gonadotropinreleasing hormone secretion is the mechanism of persistent anovulation. $J$ Clin Endocrinol Metab 1985; 61: 851-8. 
31. Powers CA, Johnson DC. Correlation of LH, FSH and GnRH release during various brain-pituitary coincubations. Hormone research 1984; 19: 117-26.

32. Byrne CJ, Fair S, English AM et al. Effect of breed, plane of nutrition and age on growth, scrotal development, metabolite concentrations and on systemic gonadotropin and testosterone concentrations following a GnRH challenge in young dairy bulls. Theriogenology 2017; 96: 58-68.

33. Veldhuis JD, Liu PY, Takahashi PY et al. Analysis of the impact of intravenous LH pulses versus continuous LH infusion on testosterone secretion during GnRHreceptor blockade. American journal of physiology Regulatory, integrative and comparative physiology 2012; 303: R994-R1002.

34. Huerta R, Malacara JM, Fajardo ME et al. High-frequency FSH and LH pulses in obese menopausal women. Endocrine 1997; 7: 281-6.

35. Balasubramanian R, Dwyer A, Seminara SB et al. Human GnRH deficiency: a unique disease model to unravel the ontogeny of GnRH neurons. Neuroendocrinology 2010; 92: 81-99.

36. Hall JE. Neuroendocrine changes with reproductive aging in women. Semin Reprod Med 2007; 25: 344-51.

37. Dunaif A. Insulin resistance and the polycystic ovary syndrome: mechanism and implications for pathogenesis. Endocr Rev 1997; 18: 774-800.

38. Dunaif A. Insulin action in the polycystic ovary syndrome. Endocrinol Metab Clin North Am 1999; 28: 341-59.

39. Seminara SB, Hayes FJ, Crowley WF, Jr. Gonadotropin-releasing hormone deficiency in the human (idiopathic hypogonadotropic hypogonadism and Kallmann's syndrome): pathophysiological and genetic considerations. Endocrine reviews 1998; 19: 521-39.

40. Laitinen EM, Vaaralahti K, Tommiska J et al. Incidence, phenotypic features and molecular genetics of Kallmann syndrome in Finland. Orphanet journal of rare diseases 2011; 6: 41.

41. Santoro N, Filicori M, Crowley WF, Jr. Hypogonadotropic disorders in men and women: diagnosis and therapy with pulsatile gonadotropin-releasing hormone. Endocrine reviews 1986; 7: 11-23.

42. Perkins RB, Hall JE, Martin KA. Neuroendocrine abnormalities in hypothalamic amenorrhea: spectrum, stability, and response to neurotransmitter modulation. The Journal of clinical endocrinology and metabolism 1999; 84: 1905-11.

43. Giusti M, Perfumo F, Verrina E et al. Delayed puberty in uremia: pituitary-gonadal function during short-term pulsatile luteinizing hormone-releasing hormone administration. Journal of endocrinological investigation 1992; 15: 709-17.

44. Dandona P, Dhindsa S, Chaudhuri A et al. Hypogonadotrophic hypogonadism in type 2 diabetes, obesity and the metabolic syndrome. Current molecular medicine 2008; 8: 816-28.

45. Skorupskaite K, George JT, Anderson RA. The kisspeptin-GnRH pathway in human reproductive health and disease. Human reproduction update 2014; 20: 485-500. 
46. Schneider F, Tomek W, Grundker C. Gonadotropin-releasing hormone (GnRH) and its natural analogues: a review. Theriogenology 2006; 66: 691-709.

47. Millar RP, Newton CL. Current and future applications of GnRH, kisspeptin and neurokinin B analogues. Nature reviews Endocrinology 2013; 9: 451-66.

48. Jayasena CN, Nijher GM, Abbara A et al. Twice-weekly administration of kisspeptin-54 for 8 weeks stimulates release of reproductive hormones in women with hypothalamic amenorrhea. Clinical pharmacology and therapeutics 2010; 88: 840-7.

49. Jayasena CN, Abbara A, Veldhuis JD et al. Increasing LH pulsatility in women with hypothalamic amenorrhoea using intravenous infusion of Kisspeptin-54. The Journal of clinical endocrinology and metabolism 2014; 99: E953-61.

50. Jayasena CN, Nijher GM, Chaudhri OB et al. Subcutaneous injection of kisspeptin54 acutely stimulates gonadotropin secretion in women with hypothalamic amenorrhea, but chronic administration causes tachyphylaxis. The Journal of clinical endocrinology and metabolism 2009; 94: 4315-23.

51. George JT, Veldhuis JD, Tena-Sempere M et al. Exploring the pathophysiology of hypogonadism in men with type 2 diabetes: kisspeptin-10 stimulates serum testosterone and LH secretion in men with type 2 diabetes and mild biochemical hypogonadism. Clinical endocrinology 2013; 79: 100-4.

52. Dhillo WS, Chaudhri OB, Patterson $M$ et al. Kisspeptin-54 stimulates the hypothalamic-pituitary gonadal axis in human males. J Clin Endocrinol Metab 2005; 90: 6609-15.

53. Nakamura S, Wakabayashi Y, Yamamura T et al. A neurokinin 3 receptor-selective agonist accelerates pulsatile luteinizing hormone secretion in lactating cattle. Biology of reproduction 2017; 97: 81-90.

54. Misu R, Oishi S, Yamada A et al. Development of novel neurokinin 3 receptor (NK3R) selective agonists with resistance to proteolytic degradation. Journal of medicinal chemistry 2014; 57: 8646-51.

55. Matsuzaki T, Tungalagsuvd A, Munkhzaya M et al. Neurokinin B receptor agonist and Dynorphin receptor antagonist stimulated luteinizing hormone secretion in fasted male rodents. Endocrine journal 2018; 65: 485-92.

56. Endo N, Rahayu LP, Ito Y, Tanaka T. Ovarian and hormonal responses to single or continuous peripheral administration of senktide, a neurokinin 3 receptor agonist, during the follicular phase in goats. Domestic animal endocrinology 2015; 53: 13643.

57. Gorkem U, Togrul C, Arslan E et al. Is there a role for kisspeptin in pathogenesis of polycystic ovary syndrome? Gynecological endocrinology : the official journal of the International Society of Gynecological Endocrinology 2018; 34: 157-60.

58. Roseweir AK, Kauffman AS, Smith JT et al. Discovery of potent kisspeptin antagonists delineate physiological mechanisms of gonadotropin regulation. The Journal of neuroscience : the official journal of the Society for Neuroscience 2009; 29: 3920-9. 
59. Prague JK, Roberts RE, Comninos AN et al. Neurokinin 3 receptor antagonism as a novel treatment for menopausal hot flushes: a phase 2, randomised, double-blind, placebo-controlled trial. Lancet 2017; 389: 1809-20.

60. Jayasena CN, Abbara A, Comninos AN et al. Kisspeptin-54 triggers egg maturation in women undergoing in vitro fertilization. The Journal of clinical investigation 2014; 124: 3667-77.

61. Kol S, Fainaru O. GnRH Agonist Triggering of Ovulation Replacing hCG: A 30Year-Old Revolution in IVF Practice Led by Rambam Health Care Campus. Rambam Maimonides medical journal 2017; 8.

62. Amsterdam JD, Maislin G, Rosenzweig M, Halbrecht U. Gonadotropin (LH and FSH) response after submaximal GnRH stimulation in depressed premenopausal women and healthy controls. Psychoneuroendocrinology 1995; 20: 311-21.

63. Moult PJ, Rees LH, Besser GM. Pulsatile gonadotrophin secretion in hyperprolactinaemic amenorrhoea an the response to bromocriptine therapy. Clinical endocrinology 1982; 16: 153-62.

64. Tay CC, Glasier AF, Illingworth PJ, Baird DT. Abnormal twenty-four hour pattern of pulsatile luteinizing hormone secretion and the response to naloxone in women with hyperprolactinaemic amenorrhoea. Clinical endocrinology 1993; 39: 599-606.

65. Bouchard P, Lagoguey M, Brailly S, Schaison G. Gonadotropin-releasing hormone pulsatile administration restores luteinizing hormone pulsatility and normal testosterone levels in males with hyperprolactinemia. The Journal of clinical endocrinology and metabolism 1985; 60: 258-62.

66. Backstrom CT, McNeilly AS, Leask RM, Baird DT. Pulsatile secretion of LH, FSH, prolactin, oestradiol and progesterone during the human menstrual cycle. Clinical endocrinology 1982; 17: 29-42.

67. Burger CW, Korsen T, van Kessel $\mathrm{H}$ et al. Pulsatile luteinizing hormone patterns in the follicular phase of the menstrual cycle, polycystic ovarian disease (PCOD) and non-PCOD secondary amenorrhea. The Journal of clinical endocrinology and metabolism 1985; 61: 1126-32.

68. Reame N, Sauder SE, Kelch RP, Marshall JC. Pulsatile gonadotropin secretion during the human menstrual cycle: evidence for altered frequency of gonadotropinreleasing hormone secretion. The Journal of clinical endocrinology and metabolism 1984; 59: 328-37.

69. Soules MR, Steiner RA, Clifton DK et al. Progesterone modulation of pulsatile luteinizing hormone secretion in normal women. The Journal of clinical endocrinology and metabolism 1984; 58: 378-83.

70. Odell WD, Ross GT, Rayford PL. Radioimmunoassay for human luteinizing hormone. Metabolism: clinical and experimental 1966; 15: 287-9.

71. Paul WE, Odell WD. Radiation Inactivation of the Immunological and Biological Activities of Human Chorionic Gonadotropin. Nature 1964; 203: 979-80.

72. Seibel MM, Levesque LA, Taymor ML. A rapid radioimmunoassay method for serum luteinizing hormone utilizing polyethylene glycol and a double-antibody method of separation. Fertility and sterility 1981; 35: 36-9. 
73. Odell WD, Griffin J. Two-monoclonal-antibody "sandwich"-type assay of human lutropin, with no cross reaction with choriogonadotropin. Clinical chemistry 1987; 33: $1603-7$.

74. Pettersson KS, Soderholm JR. Ultrasensitive two-site immunometric assay of human lutropin by time-resolved fluorometry. Clinical chemistry 1990; 36: 192833.

75. Wheeler MJ. Automated immunoassay analysers. Ann Clin Biochem 2001; 38 217-29.

76. Wheeler MJ. The measurement of LH, FSH, and prolactin. Methods Mol Biol 2013; 1065: $105-16$.

77. Juntao L, Zhuang K, Yang $\mathrm{W}$ et al. A rapid quantitative determination method of Luteinizing hormone with gold immunochromatographic strip. Conference proceedings : Annual International Conference of the IEEE Engineering in Medicine and Biology Society IEEE Engineering in Medicine and Biology Society Annual Conference 2017; 2017: 17-20.

78. Yen SS, Tsai CC, Naftolin F et al. Pulsatile patterns of gonadotropin release in subjects with and without ovarian function. The Journal of clinical endocrinology and metabolism 1972; 34: 671-5.

79. Merriam GR, Wachter KW. Algorithms for the study of episodic hormone secretion. The American journal of physiology 1982; 243: E310-8.

80. Clifton DK, Steiner RA. Cycle detection: a technique for estimating the frequency and amplitude of episodic fluctuations in blood hormone and substrate concentrations. Endocrinology 1983; 112: 1057-64.

81. Evans WS, Sollenberger MJ, Booth RA, Jr. et al. Contemporary aspects of discrete peak-detection algorithms. II. The paradigm of the luteinizing hormone pulse signal in women. Endocrine reviews 1992; 13: 81-104.

82. Oerter KE, Guardabasso V, Rodbard D. Detection and characterization of peaks and estimation of instantaneous secretory rate for episodic pulsatile hormone secretion. Computers and biomedical research, an international journal 1986; 19: 170-91.

83. Veldhuis JD, Johnson ML. Cluster analysis: a simple, versatile, and robust algorithm for endocrine pulse detection. The American journal of physiology 1986; 250: E486-93.

84. Liu PY, Keenan DM, Kok P et al. Sensitivity and specificity of pulse detection using a new deconvolution method. Am J Physiol-Endoc M 2009; 297: E538-E44.

85. Genazzani AD, Rodbard D, Forti G et al. Estimation of Instantaneous Secretory Rate of Luteinizing-Hormone in Women during the Menstrual-Cycle and in Men. Clinical endocrinology 1990; 32: 573-81.

86. Veldhuis JD, Carlson ML, Johnson ML. The Pituitary-Gland Secretes in Bursts Appraising the Nature of Glandular Secretory Impulses by Simultaneous MultipleParameter Deconvolution of Plasma-Hormone Concentrations. Proceedings of the National Academy of Sciences of the United States of America 1987; 84: 7686-90.

87. Johnson ML, Pipes L, Veldhuis PP et al. AutoDecon, a deconvolution algorithm for identification and characterization of luteinizing hormone secretory bursts: 
Description and validation using synthetic data. Analytical biochemistry 2008; 381: 8-17.

88. Liu HY, Polotsky AJ, Grunwald GK, Carlson NE. Bayesian analysis improves pulse secretion characterization in reproductive hormones. Syst Biol Reprod Med 2018; 64: 80-91.

89. Ellington AD, Szostak JW. In vitro selection of RNA molecules that bind specific ligands. Nature 1990; 346: 818-22.

90. Tuerk C, Gold L. Systematic evolution of ligands by exponential enrichment: RNA ligands to bacteriophage T4 DNA polymerase. Science 1990; 249: 505-10.

91. Jo H, Ban C. Aptamer-nanoparticle complexes as powerful diagnostic and therapeutic tools. Experimental \& molecular medicine 2016; 48: e230.

92. Zhu Q, Liu G, Kai M. DNA Aptamers in the Diagnosis and Treatment of Human Diseases. Molecules 2015; 20: 20979-97.

93. Kimoto M, Matsunaga KI, Hirao I. Evolving Aptamers with Unnatural Base Pairs. Current protocols in chemical biology 2017; 9: 315-39.

94. Zhuo Z, Yu Y, Wang M et al. Recent Advances in SELEX Technology and Aptamer Applications in Biomedicine. International journal of molecular sciences 2017; 18.

95. Pristoupil TI, Kramlova M. Microchromatographic separation of ribonucleic acids from proteins on nitrocellulose membranes. Journal of chromatography 1968; 32: 769-70.

96. Cheung YW, Kwok J, Law AW et al. Structural basis for discriminatory recognition of Plasmodium lactate dehydrogenase by a DNA aptamer. Proceedings of the National Academy of Sciences of the United States of America 2013; 110: 15967-72.

97. Tucker WO, Kinghorn AB, Fraser LA et al. Selection and Characterization of a DNA Aptamer Specifically Targeting Human HECT Ubiquitin Ligase WWP1. International journal of molecular sciences 2018; 19.

98. Shum KT, Tanner JA. Differential inhibitory activities and stabilisation of DNA aptamers against the SARS coronavirus helicase. Chembiochem : a European journal of chemical biology 2008; 9: 3037-45.

99. Shum KT, Lui EL, Wong SC et al. Aptamer-mediated inhibition of Mycobacterium tuberculosis polyphosphate kinase 2. Biochemistry 2011; 50: 3261-71.

100. Vinores SA. Technology evaluation: pegaptanib, Eyetech/Pfizer. Current opinion in molecular therapeutics 2003; 5: 673-9.

101. Gupta S, Drolet DW, Wolk SK et al. Pharmacokinetic Properties of DNA Aptamers with Base Modifications. Nucleic acid therapeutics 2017; 27: 345-53.

102. Ding F, Gao Y, He X. Recent progresses in biomedical applications of aptamerfunctionalized systems. Bioorganic \& medicinal chemistry letters 2017; 27: 425669.

103. Zhuo ZJ, Yu YY, Wang ML et al. Recent Advances in SELEX Technology and Aptamer Applications in Biomedicine. International journal of molecular sciences $2017 ; 18$. 
104. Ikebukuro K, Kiyohara C, Sode K. Novel electrochemical sensor system for protein using the aptamers in sandwich manner. Biosensors \& bioelectronics 2005; 20: $2168-72$.

105. Ding C, Ge Y, Lin JM. Aptamer based electrochemical assay for the determination of thrombin by using the amplification of the nanoparticles. Biosensors \& bioelectronics 2010; 25: 1290-4.

106. Abbaspour A, Norouz-Sarvestani F, Noori A, Soltani N. Aptamer-conjugated silver nanoparticles for electrochemical dual-aptamer-based sandwich detection of staphylococcus aureus. Biosensors \& bioelectronics 2015; 68: 149-55.

107. Zhu B, Alsager OA, Kumar S et al. Label-free electrochemical aptasensor for femtomolar detection of 17beta-estradiol. Biosensors \& bioelectronics 2015; 70: 398-403.

108. Xiao Y, Lai RY, Plaxco KW. Preparation of electrode-immobilized, redoxmodified oligonucleotides for electrochemical DNA and aptamer-based sensing. Nature protocols 2007; 2: 2875-80.

109. Dauphin-Ducharme P, Plaxco KW. Maximizing the Signal Gain of Electrochemical-DNA Sensors. Analytical chemistry 2016; 88: 11654-62.

110. Ferguson BS, Hoggarth DA, Maliniak D et al. Real-time, aptamer-based tracking of circulating therapeutic agents in living animals. Science translational medicine 2013; 5: 213 ra165.

111. Meirinho SG, Dias LG, Peres AM, Rodrigues LR. Voltammetric aptasensors for protein disease biomarkers detection: A review. Biotechnol Adv 2016; 34: 941-53.

112. Chang AL, McKeague M, Smolke CD. Facile characterization of aptamer kinetic and equilibrium binding properties using surface plasmon resonance. Methods in enzymology 2014; 549: 451-66.

113. Amano R, Takada K, Tanaka Y et al. Kinetic and Thermodynamic Analyses of Interaction between a High-Affinity RNA Aptamer and Its Target Protein. Biochemistry 2016; 55: 6221-9.

114. Entzian C, Schubert T. Studying small molecule-aptamer interactions using MicroScale Thermophoresis (MST). Methods 2016; 97: 27-34.

115. Zuker M. Mfold web server for nucleic acid folding and hybridization prediction. Nucleic acids research 2003; 31: 3406-15.

116. Drescher DG, Selvakumar D, Drescher MJ. Analysis of Protein Interactions by Surface Plasmon Resonance. Advances in protein chemistry and structural biology 2018; 110: 1-30.

117. Del Villar-Guerra R, Trent JO, Chaires JB. G-Quadruplex Secondary Structure Obtained from Circular Dichroism Spectroscopy. Angewandte Chemie 2017.

118. Wolf LK, Gao Y, Georgiadis RM. Kinetic discrimination of sequence-specific DNA - Drug binding measured by surface plasmon resonance imaging and comparison to solution-phase measurements. Journal of the American Chemical Society 2007; 129: 10503-11.

119. Wu ZS, Guo MM, Zhang SB et al. Reusable electrochemical sensing platform for highly sensitive detection of small molecules based on structure-switching signaling aptamers. Analytical chemistry 2007; 79: 2933-9. 
120. Schoukroun-Barnes LR, Macazo FC, Gutierrez B et al. Reagentless, StructureSwitching, Electrochemical Aptamer-Based Sensors. Annu Rev Anal Chem (Palo Alto Calif) 2016; 9: 163-81.

121. Hauke A, Kumar LSS, Kim MY et al. Superwetting and aptamer functionalized shrink-induced high surface area electrochemical sensors. Biosensors \& bioelectronics 2017; 94: 438-42.

122. Li H, Dauphin-Ducharme $\mathrm{P}$, Arroyo-Curras $\mathrm{N}$ et al. A Biomimetic Phosphatidylcholine-Terminated Monolayer Greatly Improves the In Vivo Performance of Electrochemical Aptamer-Based Sensors. Angewandte Chemie 2017; 56: 7492-5.

123. Arroyo-Curras N, Scida K, Ploense KL et al. High Surface Area Electrodes Generated via Electrochemical Roughening Improve the Signaling of Electrochemical Aptamer-Based Biosensors. Analytical chemistry 2017; 89: 12185-91.

124. Arroyo-Curras N, Somerson J, Vieira PA et al. Real-time measurement of small molecules directly in awake, ambulatory animals. Proceedings of the National Academy of Sciences of the United States of America 2017; 114: 645-50.

125. Farid S, Meshik X, Choi M et al. Detection of Interferon gamma using graphene and aptamer based FET-like electrochemical biosensor. Biosensors \& bioelectronics 2015; 71: 294-9.

126. Li Y, Wang Q, Zhang Y et al. A label-free electrochemical aptasensor based on graphene oxide/double-stranded DNA nanocomposite. Colloids and surfaces B, Biointerfaces 2016; 145: 160-6.

127. Balamurugan S, Obubuafo A, Soper SA et al. Designing highly specific biosensing surfaces using aptamer monolayers on gold. Langmuir: the ACS journal of surfaces and colloids 2006; 22: 6446-53.

128. Laviron E. General expression of the linear potential sweep voltammogram in the case of diffusionless electrochemical systems. J Electroanal Chem 1979; 101: 1928.

129. Farjami E, Campos R, Ferapontova EE. Effect of the DNA end of tethering to electrodes on electron transfer in methylene blue-labeled DNA duplexes. Langmuir : the ACS journal of surfaces and colloids 2012; 28: 16218-26.

130. Yu ZG, Lai RY. A reagentless and reusable electrochemical aptamer-based sensor for rapid detection of ampicillin in complex samples. Talanta 2018; 176: 619-24.

131. Yang Z, Gonzalezcortes A, Jourquin G et al. Analytical Application of SelfAssembled Monolayers on Gold Electrodes - Critical Importance of Surface Pretreatment. Biosensors \& bioelectronics 1995; 10: 789-95.

132. Dirkzwager RM, Liang SL, Tanner JA. Development of Aptamer-Based Point-ofCare Diagnostic Devices for Malaria Using Three-Dimensional Printing Rapid Prototyping. Acs Sensors 2016; 1: 420-6.

133. Dirkzwager RM, Kinghorn AB, Richards JS, Tanner JA. APTEC: aptamertethered enzyme capture as a novel rapid diagnostic test for malaria. Chemical communications 2015; 51: 4697-700. 
134. Farrington AM, Jagota N, Slater JM. Simple solid wire microdisc electrodes for the determination of vitamin C in fruit juices. The Analyst 1994; 119: 233-8.

135. Comninos AN, Wall MB, Demetriou L et al. Kisspeptin modulates sexual and emotional brain processing in humans. J Clin Invest 2017; 127: 709-19.

136. Vimalesvaran S, Narayanaswamy S, Yang L et al. Using kisspeptin to assess $\mathrm{GnRH}$ function in an unusual case of primary amenorrhoea. Endocrinol Diabetes Metab Case Rep 2017; 2017.

137. Abbara A, Clarke S, Islam R et al. A second dose of kisspeptin-54 improves oocyte maturation in women at high risk of ovarian hyperstimulation syndrome: a Phase 2 randomized controlled trial. Hum Reprod 2017; 32: 1915-24.

138. Chambers ES, Viardot A, Psichas A et al. Effects of targeted delivery of propionate to the human colon on appetite regulation, body weight maintenance and adiposity in overweight adults. Gut 2015; 64: 1744-54.

139. Narayanaswamy S, Prague JK, Jayasena $\mathrm{CN}$ et al. Investigating the KNDy Hypothesis in Humans by Coadministration of Kisspeptin, Neurokinin B, and Naltrexone in Men. The Journal of clinical endocrinology and metabolism 2016; 101: 3429-36.

140. Narayanaswamy S, Jayasena $\mathrm{CN}, \mathrm{Ng} \mathrm{N}$ et al. Subcutaneous infusion of kisspeptin54 stimulates gonadotrophin release in women and the response correlates with basal oestradiol levels. Clinical endocrinology 2016; 84: 939-45.

141. Tata JR. One hundred years of hormones. EMBO reports 2005; 6: 490-6.

142. He W, Li X, Adekunbi D et al. Hypothalamic effects of progesterone on regulation of the pulsatile and surge release of luteinising hormone in female rats. Scientific reports 2017; 7: 8096.

143. Li SY, Li XF, Hu MH et al. Neurokinin B receptor antagonism decreases luteinising hormone pulse frequency and amplitude and delays puberty onset in the female rat. Journal of neuroendocrinology 2014; 26: 521-7.

144. Chang $\mathrm{CC}$, Chen $\mathrm{CP}$, Lee $\mathrm{CH}$ et al. Colorimetric detection of human chorionic gonadotropin using catalytic gold nanoparticles and a peptide aptamer. Chemical communications 2014; 50: 14443-6.

145. Lin JS, McNatty KP. Aptamer-based regionally protected PCR for protein detection. Clinical chemistry 2009; 55: 1686-93.

146. Chang AL, McKeague M, Liang JC, Smolke CD. Kinetic and equilibrium binding characterization of aptamers to small molecules using a label-free, sensitive, and scalable platform. Analytical chemistry 2014; 86: 3273-8.

147. Latham MP, Zimmermann GR, Pardi A. NMR chemical exchange as a probe for ligand-binding kinetics in a theophylline-binding RNA aptamer. Journal of the American Chemical Society 2009; 131: 5052-3.

148. Hoogvliet JC, Dijksma M, Kamp B, van Bennekom WP. Electrochemical pretreatment of polycrystalline gold electrodes to produce a reproducible surface roughness for self-assembly: a study in phosphate buffer $\mathrm{pH}$ 7.4. Analytical chemistry 2000; 72: 2016-21. 
149. Shervedani RK, Babadi MK. Application of 2-mercaptobenzothiazole selfassembled monolayer on polycrystalline gold electrode as a nanosensor for determination of $\mathrm{Ag}(\mathrm{I})$. Talanta 2006; 69: 741-6.

150. Le Floch F, Ho HA, Leclerc M. Label-free electrochemical detection of protein based on a ferrocene-bearing cationic polythiophene and aptamer. Analytical chemistry 2006; 78: 4727-31.

151. Lu Y, Zhu N, Yu P, Mao L. Aptamer-based electrochemical sensors that are not based on the target binding-induced conformational change of aptamers. The Analyst 2008; 133: 1256-60.

152. R P. Decomposition of the ferricenium cation by nucleophilic reagents. $J$ Organomet Chem 1972; 39. 


\section{Appendix}


Request letters for copyright permission 


\title{
Request to reproduce a figure from a paper for my PhD thesis
}

\author{
Liang, Shaolin
}

周日 2018/9/23 15:37

收件人:subscriptions@the-aps.org <subscriptions@the-aps.org>;

Dear American Physiological Society,

I have completed my PhD thesis at Imperial College London entitled 'An aptamer-based

sensing platform for luteinising hormone pulsatility measurement'.

I seek your permission to reprint, in my thesis an figure from:

Keenan DM, Veldhuis JD. Pulsatility of Hypothalamo-Pituitary Hormones: A Challenge in Quantification. Physiology (Bethesda) 2016; 31: 34-50.

The Figure to be reproduced is attached in this email.

I would like to include the figure in my thesis which will be added to Spiral, Imperial's institutional repositoryhttp://spiral.imperial.ac.uk/

and made available to the public under a Creative Commons Attribution-NonCommercial-NoDerivs licence.

If you are happy to grant me all the permissions requested, please return a signed copy of this letter. If you wish to grant only some of the permissions requested, please list these and then sign.

Yours sincerely,

Shaolin Liang

\section{Permission granted for the use requested above:}

I confirm that I am the copyright holder of the extract above and hereby give permission to include it in your thesis which will be made available, via the internet, for non-commercial purposes under the terms of the user licence.

Signed:

Name:

Organisation:

Job title: 

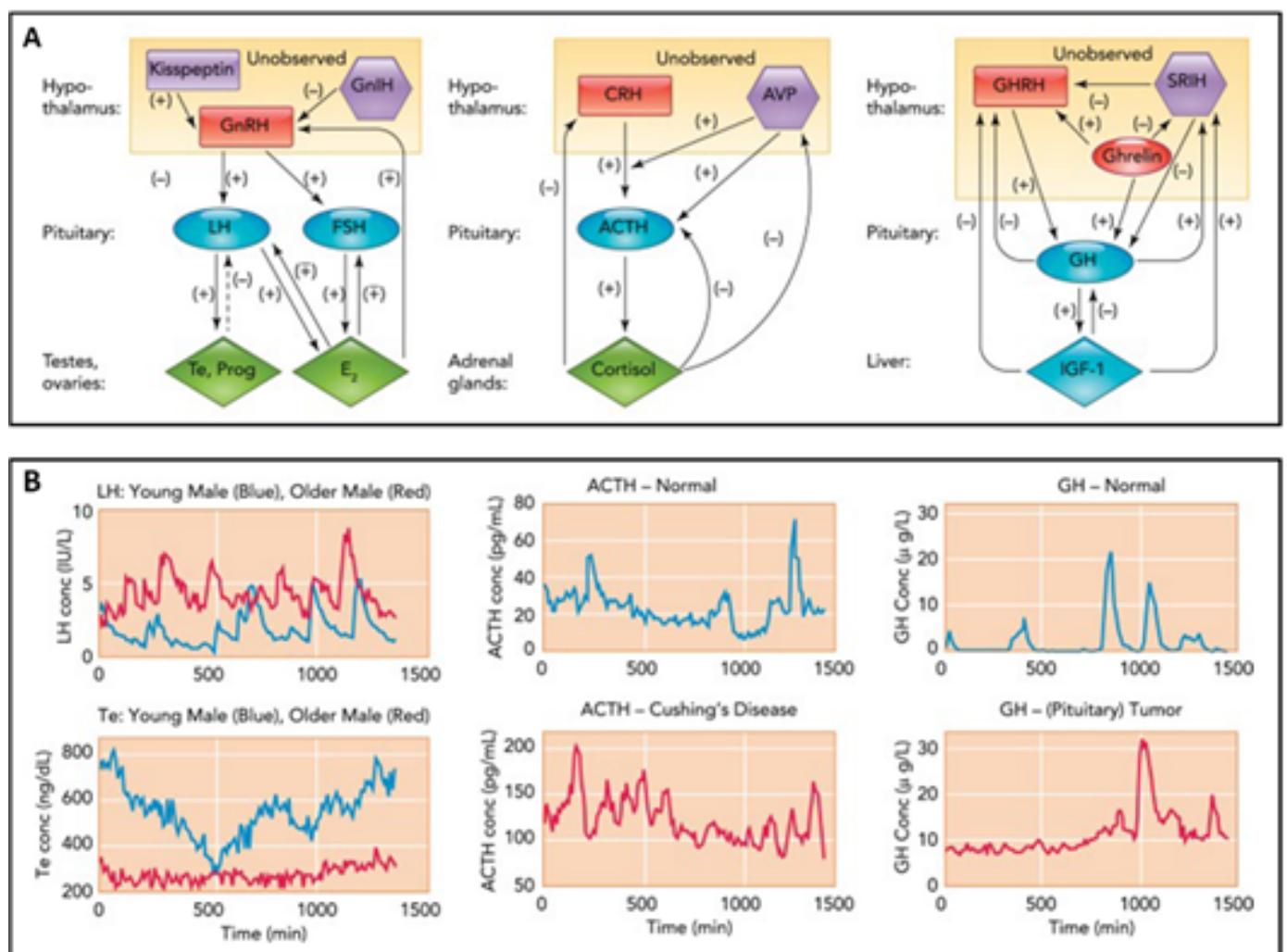


\title{
Request to reproduce a figure from a paper for my PhD thesis
}

\author{
Liang, Shaolin
}

周日 2018/9/23 15:34

收件人:journals.permissions@oup.com <journals.permissions@oup.com>;

Dear The Endocrine Society,

I have completed my PhD thesis at Imperial College London entitled 'An aptamer-based

\section{sensing platform for luteinising hormone pulsatility measurement'.}

I seek your permission to reprint, in my thesis a figure from:

Dierschke DJ, Bhattacharya AN, Atkinson LE, Knobil E. Circhoral oscillations of plasma LH levels in the ovariectomized rhesus monkey. Endocrinology 1970; 87: 850-3.

The Figure to be reproduced is attached in this email.

I would like to include the figure in my thesis which will be added to Spiral, Imperial's institutional repository http://spiral.imperial.ac.uk/

and made available to the public under a Creative Commons Attribution-NonCommercial-NoDerivs licence.

If you are happy to grant me all the permissions requested, please return a signed copy of this letter. If you wish to grant only some of the permissions requested, please list these and then sign. Yours sincerely,

Shaolin Liang

\section{Permission granted for the use requested above:}

I confirm that I am the copyright holder of the extract above and hereby give permission to include it in your thesis which will be made available, via the internet, for non-commercial purposes under the terms of the user licence.

Signed:

Name:

Organisation:

Job title:
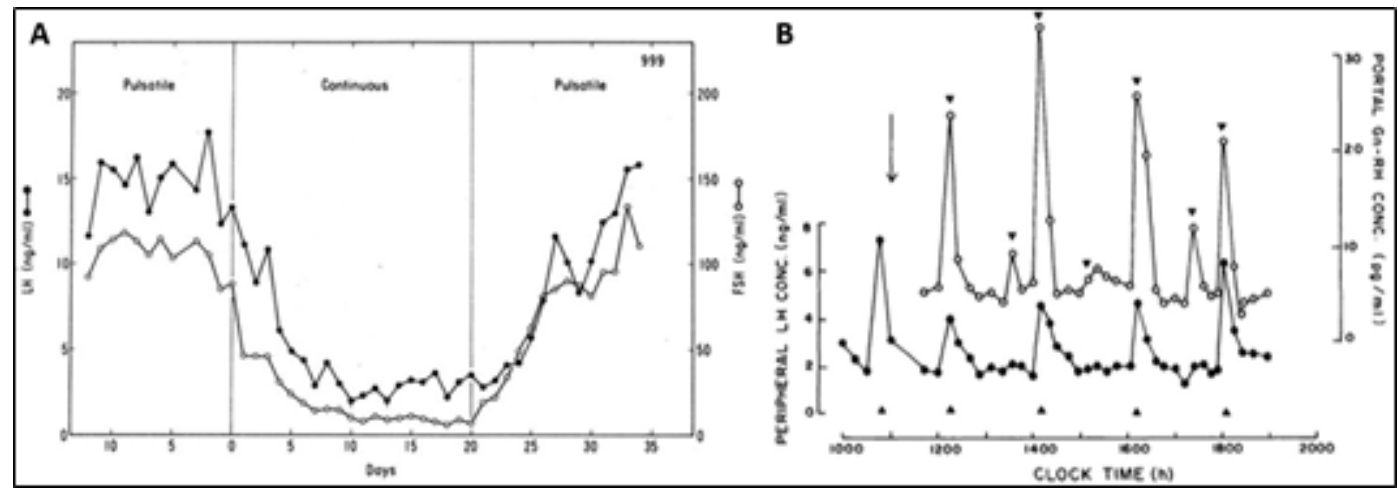
This Agreement between Imperial College London ("You") and The American Association for the Advancement of Science ("The American Association for the Advancement of Science") consists of your license details and the terms and conditions provided by The American Association for the Advancement of Science and Copyright Clearance Center.

License Number

License date

Licensed Content Publisher

Licensed Content Publication Science

Licensed Content Title

Licensed Content Author

Licensed Content Date

Licensed Content Volume

Licensed Content Issue

Volume number

Issue number

Type of Use

Requestor type

Format

Portion

Number of figures/tables

Order reference number

Title of your thesis / dissertation

Expected completion date

Estimated size(pages)

Requestor Location

Billing Type

Billing Address

Total

Terms and Conditions

202

4368

202

4368

Figure

1

200

Invoice
4434821477070

Sep 23, 2018

The American Association for the Advancement of Science

Hypophysial responses to continuous and intermittent delivery of hypopthalamic gonadotropin-releasing hormone

PE Belchetz,TM Plant, Y Nakai,EJ Keogh,E Knobil

Nov 10, 1978

Thesis / Dissertation

Author of the AAAS published paper

Print and electronic

An aptamer-based sensing platform for luteinising hormone pulsatility measurement

Sep 2018

Imperial College London Imperial College London

London, SW7 2AZ

United Kingdom

Attn: Imperial College London

Imperial College London

Imperial College London

London, United Kingdom SW7 2AZ

Attn: Imperial College London

$0.00 \mathrm{GBP}$

American Association for the Advancement of Science TERMS AND CONDITIONS 
Regarding your request, we are pleased to grant you non-exclusive, non-transferable permission, to republish the AAAS material identified above in your work identified above, subject to the terms and conditions herein. We must be contacted for permission for any uses other than those specifically identified in your request above.

The following credit line must be printed along with the AAAS material: "From [Full Reference Citation]. Reprinted with permission from AAAS."

All required credit lines and notices must be visible any time a user accesses any part of the AAAS material and must appear on any printed copies and authorized user might make. This permission does not apply to figures / photos / artwork or any other content or materials included in your work that are credited to non-AAAS sources. If the requested material is sourced to or references non-AAAS sources, you must obtain authorization from that source as well before using that material. You agree to hold harmless and indemnify AAAS against any claims arising from your use of any content in your work that is credited to non-AAAS sources.

If the AAAS material covered by this permission was published in Science during the years 1974 - 1994, you must also obtain permission from the author, who may grant or withhold permission, and who may or may not charge a fee if permission is granted. See original article for author's address. This condition does not apply to news articles.

The AAAS material may not be modified or altered except that figures and tables may be modified with permission from the author. Author permission for any such changes must be secured prior to your use.

Whenever possible, we ask that electronic uses of the AAAS material permitted herein include a hyperlink to the original work on AAAS's website (hyperlink may be embedded in the reference citation).

AAAS material reproduced in your work identified herein must not account for more than $30 \%$ of the total contents of that work.

AAAS must publish the full paper prior to use of any text.

AAAS material must not imply any endorsement by the American Association for the Advancement of Science.

This permission is not valid for the use of the AAAS and/or Science logos.

AAAS makes no representations or warranties as to the accuracy of any information contained in the AAAS material covered by this permission, including any warranties of merchantability or fitness for a particular purpose.

If permission fees for this use are waived, please note that AAAS reserves the right to charge for reproduction of this material in the future.

Permission is not valid unless payment is received within sixty (60) days of the issuance of this permission. If payment is not received within this time period then all rights granted herein shall be revoked and this permission will be considered null and void.

In the event of breach of any of the terms and conditions herein or any of CCC's Billing and Payment terms and conditions, all rights granted herein shall be revoked and this permission will be considered null and void.

AAAS reserves the right to terminate this permission and all rights granted herein at its discretion, for any purpose, at any time. In the event that AAAS elects to terminate this permission, you will have no further right to publish, publicly perform, publicly display, distribute or otherwise use any matter in which the AAAS content had been included, and all fees paid hereunder shall be fully refunded to you. Notification of termination will be sent to the contact information as supplied by you during the request process and termination shall be immediate upon sending the notice. Neither AAAS nor CCC shall be liable for any costs, expenses, or damages you may incur as a result of the termination of this permission, beyond the refund noted above.

This Permission may not be amended except by written document signed by both parties. The terms above are applicable to all permissions granted for the use of AAAS material. Below you will find additional conditions that apply to your particular type of use.

\section{FOR A THESIS OR DISSERTATION}

If you are using figure(s)/table(s), permission is granted for use in print and electronic versions of your dissertation or thesis. A full text article may be used in print versions only of a dissertation or thesis. 
Permission covers the distribution of your dissertation or thesis on demand by ProQuest / UMI, provided the AAAS material covered by this permission remains in situ.

If you are an Original Author on the AAAS article being reproduced, please refer to your License to Publish for rules on reproducing your paper in a dissertation or thesis.

\section{FOR JOURNALS:}

Permission covers both print and electronic versions of your journal article, however the AAAS material may not be used in any manner other than within the context of your article.

\section{FOR BOOKS/TEXTBOOKS:}

If this license is to reuse figures/tables, then permission is granted for non-exclusive world rights in all languages in both print and electronic formats (electronic formats are defined below).

If this license is to reuse a text excerpt or a full text article, then permission is granted for non-exclusive world rights in English only. You have the option of securing either print or electronic rights or both, but electronic rights are not automatically granted and do garner additional fees. Permission for translations of text excerpts or full text articles into other languages must be obtained separately.

Licenses granted for use of AAAS material in electronic format books/textbooks are valid only in cases where the electronic version is equivalent to or substitutes for the print version of the book/textbook. The AAAS material reproduced as permitted herein must remain in situ and must not be exploited separately (for example, if permission covers the use of a full text article, the article may not be offered for access or for purchase as a stand-alone unit), except in the case of permitted textbook companions as noted below.

You must include the following notice in any electronic versions, either adjacent to the reprinted AAAS material or in the terms and conditions for use of your electronic products:

"Readers may view, browse, and/or download material for temporary copying purposes only, provided these uses are for noncommercial personal purposes. Except as provided by law, this material may not be further reproduced, distributed, transmitted, modified, adapted, performed, displayed, published, or sold in whole or in part, without prior written permission from the publisher."

If your book is an academic textbook, permission covers the following companions to your textbook, provided such companions are distributed only in conjunction with your textbook at no additional cost to the user:

- Password-protected website

- Instructor's image CD/DVD and/or PowerPoint resource

- Student CD/DVD

All companions must contain instructions to users that the AAAS material may be used for non-commercial, classroom purposes only. Any other uses require the prior written permission from AAAS.

If your license is for the use of AAAS Figures/Tables, then the electronic rights granted herein permit use of the Licensed Material in any Custom Databases that you distribute the electronic versions of your textbook through, so long as the Licensed Material remains within the context of a chapter of the title identified in your request and cannot be downloaded by a user as an independent image file.

Rights also extend to copies/files of your Work (as described above) that you are required to provide for use by the visually and/or print disabled in compliance with state and federal laws.

This permission only covers a single edition of your work as identified in your request.

\section{FOR NEWSLETTERS:}

Permission covers print and/or electronic versions, provided the AAAS material reproduced as permitted herein remains in situ and is not exploited separately (for example, if permission covers the use of a full text article, the article may not be offered for access or for purchase as a stand-alone unit)

\section{FOR ANNUAL REPORTS:}

Permission covers print and electronic versions provided the AAAS material reproduced as permitted herein remains in situ and is not exploited separately (for example, if permission covers the use of a full text article, the article may not be offered for access or for purchase as a stand-alone unit) 


\section{FOR PROMOTIONAL/MARKETING USES:}

Permission covers the use of AAAS material in promotional or marketing pieces such as information packets, media kits, product slide kits, brochures, or flyers limited to a single print run. The AAAS Material may not be used in any manner which implies endorsement or promotion by the American Association for the Advancement of Science (AAAS) or Science of any product or service. AAAS does not permit the reproduction of its name, logo or text on promotional literature.

If permission to use a full text article is permitted, The Science article covered by this permission must not be altered in any way. No additional printing may be set onto an article copy other than the copyright credit line required above. Any alterations must be approved in advance and in writing by AAAS. This includes, but is not limited to, the placement of sponsorship identifiers, trademarks, logos, rubber stamping or self-adhesive stickers onto the article copies.

Additionally, article copies must be a freestanding part of any information package (i.e. media kit) into which they are inserted. They may not be physically attached to anything, such as an advertising insert, or have anything attached to them, such as a sample product. Article copies must be easily removable from any kits or informational packages in which they are used. The only exception is that article copies may be inserted into three-ring binders.

\section{FOR CORPORATE INTERNAL USE:}

The AAAS material covered by this permission may not be altered in any way. No additional printing may be set onto an article copy other than the required credit line. Any alterations must be approved in advance and in writing by AAAS. This includes, but is not limited to the placement of sponsorship identifiers, trademarks, logos, rubber stamping or self-adhesive stickers onto article copies.

If you are making article copies, copies are restricted to the number indicated in your request and must be distributed only to internal employees for internal use.

If you are using AAAS Material in Presentation Slides, the required credit line must be visible on the slide where the AAAS material will be reprinted

If you are using AAAS Material on a CD, DVD, Flash Drive, or the World Wide Web, you must include the following notice in any electronic versions, either adjacent to the reprinted AAAS material or in the terms and conditions for use of your electronic products: "Readers may view, browse, and/or download material for temporary copying purposes only, provided these uses are for noncommercial personal purposes. Except as provided by law, this material may not be further reproduced, distributed, transmitted, modified, adapted, performed, displayed, published, or sold in whole or in part, without prior written permission from the publisher." Access to any such CD, DVD, Flash Drive or Web page must be restricted to your organization's employees only.

\section{FOR CME COURSE and SCIENTIFIC SOCIETY MEETINGS:}

Permission is restricted to the particular Course, Seminar, Conference, or Meeting indicated in your request. If this license covers a text excerpt or a Full Text Article, access to the reprinted AAAS material must be restricted to attendees of your event only (if you have been granted electronic rights for use of a full text article on your website, your website must be password protected, or access restricted so that only attendees can access the content on your site).

If you are using AAAS Material on a CD, DVD, Flash Drive, or the World Wide Web, you must include the following notice in any electronic versions, either adjacent to the reprinted AAAS material or in the terms and conditions for use of your electronic products: "Readers may view, browse, and/or download material for temporary copying purposes only, provided these uses are for noncommercial personal purposes. Except as provided by law, this material may not be further reproduced, distributed, transmitted, modified, adapted, performed, displayed, published, or sold in whole or in part, without prior written permission from the publisher."

\section{FOR POLICY REPORTS:}

These rights are granted only to non-profit organizations and/or government agencies. Permission covers print and electronic versions of a report, provided the required credit line appears in both versions and provided the AAAS material reproduced as permitted herein remains in situ and is not exploited separately. 


\section{FOR CLASSROOM PHOTOCOPIES:}

Permission covers distribution in print copy format only. Article copies must be freestanding and not part of a course pack. They may not be physically attached to anything or have anything attached to them.

\section{FOR COURSEPACKS OR COURSE WEBSITES:}

These rights cover use of the AAAS material in one class at one institution. Permission is valid only for a single semester after which the AAAS material must be removed from the Electronic Course website, unless new permission is obtained for an additional semester. If the material is to be distributed online, access must be restricted to students and instructors enrolled in that particular course by some means of password or access control.

\section{FOR WEBSITES:}

You must include the following notice in any electronic versions, either adjacent to the reprinted AAAS material or in the terms and conditions for use of your electronic products: "Readers may view, browse, and/or download material for temporary copying purposes only, provided these uses are for noncommercial personal purposes. Except as provided by law, this material may not be further reproduced, distributed, transmitted, modified, adapted, performed, displayed, published, or sold in whole or in part, without prior written permission from the publisher."

Permissions for the use of Full Text articles on third party websites are granted on a case by case basis and only in cases where access to the AAAS Material is restricted by some means of password or access control. Alternately, an E-Print may be purchased through our reprints department (brocheleau@rockwaterinc.com).

REGARDING FULL TEXT ARTICLE USE ON THE WORLD WIDE WEB IF YOU ARE AN 'ORIGINAL AUTHOR' OF A SCIENCE PAPER

If you chose "Original Author" as the Requestor Type, you are warranting that you are one of authors listed on the License Agreement as a "Licensed content author" or that you are acting on that author's behalf to use the Licensed content in a new work that one of the authors listed on the License Agreement as a "Licensed content author" has written. Original Authors may post the 'Accepted Version' of their full text article on their personal or on their University website and not on any other website. The 'Accepted Version' is the version of the paper accepted for publication by AAAS including changes resulting from peer review but prior to AAAS's copy editing and production (in other words not the AAAS published version).

\section{FOR MOVIES / FILM / TELEVISION:}

Permission is granted to use, record, film, photograph, and/or tape the AAAS material in connection with your program/film and in any medium your program/film may be shown or heard, including but not limited to broadcast and cable television, radio, print, world wide web, and videocassette.

The required credit line should run in the program/film's end credits.

\section{FOR MUSEUM EXHIBITIONS:}

Permission is granted to use the AAAS material as part of a single exhibition for the duration of that exhibit. Permission for use of the material in promotional materials for the exhibit must be cleared separately with AAAS (please contact us at permissions@aaas.org).

\section{FOR TRANSLATIONS:}

Translation rights apply only to the language identified in your request summary above. The following disclaimer must appear with your translation, on the first page of the article, after the credit line: "This translation is not an official translation by AAAS staff, nor is it endorsed by AAAS as accurate. In crucial matters, please refer to the official Englishlanguage version originally published by AAAS."

\section{FOR USE ON A COVER:}

Permission is granted to use the AAAS material on the cover of a journal issue, newsletter issue, book, textbook, or annual report in print and electronic formats provided the AAAS material reproduced as permitted herein remains in situ and is not exploited separately By using the AAAS Material identified in your request, you agree to abide by all the terms and conditions herein.

Questions about these terms can be directed to the AAAS Permissions department permissions@ $\underline{\text { aaas.org. }}$ 
V 2

Questions? customercare@copyright.com or +1-855-239-3415 (toll free in the US) or +1-978-646-2777. 


\title{
Request to reproduce a figure from a paper for my PhD thesis
}

\author{
Liang, Shaolin
}

周日 2018/9/23 16:05

收件人:journals.permissions@oup.com <journals.permissions@oup.com>;

Dear The Endocrine Society,

I have completed my PhD thesis at Imperial College London entitled 'An aptamer-based sensing platform for luteinising hormone pulsatility measurement'.

I seek your permission to reprint, in my thesis a figure from:

Veldhuis JD, Carlson ML, Johnson ML. The Pituitary-Gland Secretes in Bursts -Appraising the Nature of Glandular Secretory Impulses by Simultaneous Multiple-Parameter Deconvolution of Plasma-Hormone Concentrations. Proceedings of the National Academy of Sciences of the United States of America 1987; 84: 7686-90.

The Figure to be reproduced is attached in this email.

I would like to include the figure in my thesis which will be added to Spiral, Imperial's institutional repository http://spiral.imperial.ac.uk/

and made available to the public under a Creative Commons Attribution-NonCommercial-NoDerivs licence.

If you are happy to grant me all the permissions requested, please return a signed copy of this letter. If you wish to grant only some of the permissions requested, please list these and then sign.

Yours sincerely,

Shaolin Liang

\section{Permission granted for the use requested above:}

I confirm that I am the copyright holder of the extract above and hereby give permission to include it in your thesis which will be made available, via the internet, for non-commercial purposes under the terms of the user licence.

Signed:

Name:

Organisation:

Job title: 


\section{ELSEVIER LICENSE \\ TERMS AND CONDITIONS}

This Agreement between Imperial College London ("You") and Elsevier ("Elsevier") consists of your license details and the terms and conditions provided by Elsevier and Copyright Clearance Center.

\begin{tabular}{|c|c|}
\hline License Number & 4434840305570 \\
\hline License date & Sep 23, 2018 \\
\hline Licensed Content Publisher & Elsevier \\
\hline Licensed Content Publication & Biotechnology Advances \\
\hline Licensed Content Title & $\begin{array}{l}\text { Voltammetric aptasensors for protein disease biomarkers detection: } \\
\text { A review }\end{array}$ \\
\hline Licensed Content Author & Sofia G. Meirinho,Luís G. Dias,António M. Peres,Lígia R. Rodrigues \\
\hline Licensed Content Date & September-October 2016 \\
\hline Licensed Content Volume & 34 \\
\hline Licensed Content Issue & 5 \\
\hline Licensed Content Pages & 13 \\
\hline Start Page & 941 \\
\hline End Page & 953 \\
\hline Type of Use & reuse in a thesis/dissertation \\
\hline $\begin{array}{l}\text { Intended publisher of new } \\
\text { work }\end{array}$ & other \\
\hline Portion & figures/tables/illustrations \\
\hline $\begin{array}{l}\text { Number of } \\
\text { figures/tables/illustrations }\end{array}$ & 1 \\
\hline Format & electronic \\
\hline $\begin{array}{l}\text { Are you the author of this } \\
\text { Elsevier article? }\end{array}$ & No \\
\hline Will you be translating? & No \\
\hline Original figure numbers & Figure 4 \\
\hline $\begin{array}{l}\text { Title of your } \\
\text { thesis/dissertation }\end{array}$ & $\begin{array}{l}\text { An aptamer-based sensing platform for luteinising hormone } \\
\text { pulsatility measurement }\end{array}$ \\
\hline Expected completion date & Sep 2018 \\
\hline $\begin{array}{l}\text { Estimated size (number of } \\
\text { pages) }\end{array}$ & 200 \\
\hline \multirow[t]{2}{*}{ Requestor Location } & $\begin{array}{l}\text { Imperial College London } \\
\text { Imperial College London }\end{array}$ \\
\hline & $\begin{array}{l}\text { London, SW7 2AZ } \\
\text { United Kingdom } \\
\text { Attn: Imperial College London }\end{array}$ \\
\hline Publisher Tax ID & GB 494627212 \\
\hline Total & 0.00 USD \\
\hline
\end{tabular}

Terms and Conditions 
1. The publisher for this copyrighted material is Elsevier. By clicking "accept" in connection with completing this licensing transaction, you agree that the following terms and conditions apply to this transaction (along with the Billing and Payment terms and conditions established by Copyright Clearance Center, Inc. ("CCC"), at the time that you opened your Rightslink account and that are available at any time at http://myaccount.copyright.com).

\section{GENERAL TERMS}

2. Elsevier hereby grants you permission to reproduce the aforementioned material subject to the terms and conditions indicated.

3. Acknowledgement: If any part of the material to be used (for example, figures) has appeared in our publication with credit or acknowledgement to another source, permission must also be sought from that source. If such permission is not obtained then that material may not be included in your publication/copies. Suitable acknowledgement to the source must be made, either as a footnote or in a reference list at the end of your publication, as follows:

"Reprinted from Publication title, Vol /edition number, Author(s), Title of article / title of chapter, Pages No., Copyright (Year), with permission from Elsevier [OR APPLICABLE SOCIETY COPYRIGHT OWNER]." Also Lancet special credit - "Reprinted from The Lancet, Vol. number, Author(s), Title of article, Pages No., Copyright (Year), with permission from Elsevier."

4. Reproduction of this material is confined to the purpose and/or media for which permission is hereby given.

5. Altering/Modifying Material: Not Permitted. However figures and illustrations may be altered/adapted minimally to serve your work. Any other abbreviations, additions, deletions and/or any other alterations shall be made only with prior written authorization of Elsevier

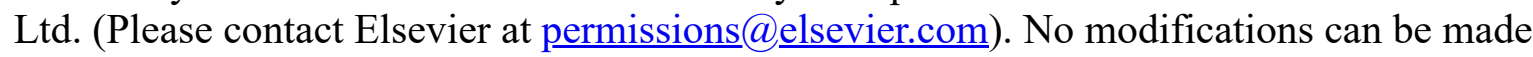
to any Lancet figures/tables and they must be reproduced in full.

6. If the permission fee for the requested use of our material is waived in this instance, please be advised that your future requests for Elsevier materials may attract a fee.

7. Reservation of Rights: Publisher reserves all rights not specifically granted in the combination of (i) the license details provided by you and accepted in the course of this licensing transaction, (ii) these terms and conditions and (iii) CCC's Billing and Payment terms and conditions.

8. License Contingent Upon Payment: While you may exercise the rights licensed immediately upon issuance of the license at the end of the licensing process for the transaction, provided that you have disclosed complete and accurate details of your proposed use, no license is finally effective unless and until full payment is received from you (either by publisher or by CCC) as provided in CCC's Billing and Payment terms and conditions. If full payment is not received on a timely basis, then any license preliminarily granted shall be deemed automatically revoked and shall be void as if never granted. Further, in the event that you breach any of these terms and conditions or any of CCC's Billing and Payment terms and conditions, the license is automatically revoked and shall be void as if never granted. Use of materials as described in a revoked license, as well as any use of the materials beyond the scope of an unrevoked license, may constitute copyright infringement and publisher reserves the right to take any and all action to protect its copyright in the materials.

9. Warranties: Publisher makes no representations or warranties with respect to the licensed material.

10. Indemnity: You hereby indemnify and agree to hold harmless publisher and CCC, and their respective officers, directors, employees and agents, from and against any and all claims arising out of your use of the licensed material other than as specifically authorized pursuant to this license.

11. No Transfer of License: This license is personal to you and may not be sublicensed, assigned, or transferred by you to any other person without publisher's written permission. 12. No Amendment Except in Writing: This license may not be amended except in a writing signed by both parties (or, in the case of publisher, by CCC on publisher's behalf).

13. Objection to Contrary Terms: Publisher hereby objects to any terms contained in any purchase order, acknowledgment, check endorsement or other writing prepared by you, which terms are inconsistent with these terms and conditions or CCC's Billing and Payment 
terms and conditions. These terms and conditions, together with CCC's Billing and Payment terms and conditions (which are incorporated herein), comprise the entire agreement between you and publisher (and CCC) concerning this licensing transaction. In the event of any conflict between your obligations established by these terms and conditions and those established by CCC's Billing and Payment terms and conditions, these terms and conditions shall control.

14. Revocation: Elsevier or Copyright Clearance Center may deny the permissions described in this License at their sole discretion, for any reason or no reason, with a full refund payable to you. Notice of such denial will be made using the contact information provided by you. Failure to receive such notice will not alter or invalidate the denial. In no event will Elsevier or Copyright Clearance Center be responsible or liable for any costs, expenses or damage incurred by you as a result of a denial of your permission request, other than a refund of the amount(s) paid by you to Elsevier and/or Copyright Clearance Center for denied permissions.

\section{LIMITED LICENSE}

The following terms and conditions apply only to specific license types: 15. Translation: This permission is granted for non-exclusive world English rights only unless your license was granted for translation rights. If you licensed translation rights you may only translate this content into the languages you requested. A professional translator must perform all translations and reproduce the content word for word preserving the integrity of the article.

16. Posting licensed content on any Website: The following terms and conditions apply as follows: Licensing material from an Elsevier journal: All content posted to the web site must maintain the copyright information line on the bottom of each image; A hyper-text must be included to the Homepage of the journal from which you are licensing at http://www.sciencedirect.com/science/journal/xxxxx or the Elsevier homepage for books at http://www.elsevier.com; Central Storage: This license does not include permission for a scanned version of the material to be stored in a central repository such as that provided by Heron/XanEdu.

Licensing material from an Elsevier book: A hyper-text link must be included to the Elsevier homepage at http://www.elsevier.com . All content posted to the web site must maintain the copyright information line on the bottom of each image.

Posting licensed content on Electronic reserve: In addition to the above the following clauses are applicable: The web site must be password-protected and made available only to bona fide students registered on a relevant course. This permission is granted for 1 year only. You may obtain a new license for future website posting.

17. For journal authors: the following clauses are applicable in addition to the above: Preprints:

A preprint is an author's own write-up of research results and analysis, it has not been peerreviewed, nor has it had any other value added to it by a publisher (such as formatting, copyright, technical enhancement etc.).

Authors can share their preprints anywhere at any time. Preprints should not be added to or enhanced in any way in order to appear more like, or to substitute for, the final versions of articles however authors can update their preprints on arXiv or RePEc with their Accepted Author Manuscript (see below).

If accepted for publication, we encourage authors to link from the preprint to their formal publication via its DOI. Millions of researchers have access to the formal publications on ScienceDirect, and so links will help users to find, access, cite and use the best available version. Please note that Cell Press, The Lancet and some society-owned have different preprint policies. Information on these policies is available on the journal homepage.

Accepted Author Manuscripts: An accepted author manuscript is the manuscript of an article that has been accepted for publication and which typically includes authorincorporated changes suggested during submission, peer review and editor-author communications.

Authors can share their accepted author manuscript:

- immediately 
- via their non-commercial person homepage or blog

$\circ$ by updating a preprint in arXiv or RePEc with the accepted manuscript

- via their research institute or institutional repository for internal institutional uses or as part of an invitation-only research collaboration work-group

- directly by providing copies to their students or to research collaborators for their personal use

- for private scholarly sharing as part of an invitation-only work group on commercial sites with which Elsevier has an agreement

- After the embargo period

- via non-commercial hosting platforms such as their institutional repository

- via commercial sites with which Elsevier has an agreement

In all cases accepted manuscripts should:

- link to the formal publication via its DOI

- bear a CC-BY-NC-ND license - this is easy to do

- if aggregated with other manuscripts, for example in a repository or other site, be shared in alignment with our hosting policy not be added to or enhanced in any way to appear more like, or to substitute for, the published journal article.

Published journal article (JPA): A published journal article (PJA) is the definitive final record of published research that appears or will appear in the journal and embodies all value-adding publishing activities including peer review co-ordination, copy-editing, formatting, (if relevant) pagination and online enrichment.

Policies for sharing publishing journal articles differ for subscription and gold open access articles:

Subscription Articles: If you are an author, please share a link to your article rather than the full-text. Millions of researchers have access to the formal publications on ScienceDirect, and so links will help your users to find, access, cite, and use the best available version. Theses and dissertations which contain embedded PJAs as part of the formal submission can be posted publicly by the awarding institution with DOI links back to the formal publications on ScienceDirect.

If you are affiliated with a library that subscribes to ScienceDirect you have additional private sharing rights for others' research accessed under that agreement. This includes use for classroom teaching and internal training at the institution (including use in course packs and courseware programs), and inclusion of the article for grant funding purposes.

Gold Open Access Articles: May be shared according to the author-selected end-user license and should contain a CrossMark logo, the end user license, and a DOI link to the formal publication on ScienceDirect.

Please refer to Elsevier's posting policy for further information.

18. For book authors the following clauses are applicable in addition to the above: Authors are permitted to place a brief summary of their work online only. You are not allowed to download and post the published electronic version of your chapter, nor may you scan the printed edition to create an electronic version. Posting to a repository: Authors are permitted to post a summary of their chapter only in their institution's repository.

19. Thesis/Dissertation: If your license is for use in a thesis/dissertation your thesis may be submitted to your institution in either print or electronic form. Should your thesis be published commercially, please reapply for permission. These requirements include permission for the Library and Archives of Canada to supply single copies, on demand, of the complete thesis and include permission for Proquest/UMI to supply single copies, on demand, of the complete thesis. Should your thesis be published commercially, please reapply for permission. Theses and dissertations which contain embedded PJAs as part of the formal submission can be posted publicly by the awarding institution with DOI links back to the formal publications on ScienceDirect.

\section{EIsevier Open Access Terms and Conditions}

You can publish open access with Elsevier in hundreds of open access journals or in nearly 2000 established subscription journals that support open access publishing. Permitted third 
party re-use of these open access articles is defined by the author's choice of Creative Commons user license. See our open access license policy for more information.

Terms \& Conditions applicable to all Open Access articles published with Elsevier: Any reuse of the article must not represent the author as endorsing the adaptation of the article nor should the article be modified in such a way as to damage the author's honour or reputation. If any changes have been made, such changes must be clearly indicated. The author(s) must be appropriately credited and we ask that you include the end user license and a DOI link to the formal publication on ScienceDirect.

If any part of the material to be used (for example, figures) has appeared in our publication with credit or acknowledgement to another source it is the responsibility of the user to ensure their reuse complies with the terms and conditions determined by the rights holder. Additional Terms \& Conditions applicable to each Creative Commons user license: CC BY: The CC-BY license allows users to copy, to create extracts, abstracts and new works from the Article, to alter and revise the Article and to make commercial use of the Article (including reuse and/or resale of the Article by commercial entities), provided the user gives appropriate credit (with a link to the formal publication through the relevant DOI), provides a link to the license, indicates if changes were made and the licensor is not represented as endorsing the use made of the work. The full details of the license are available at http://creativecommons.org/licenses/by/4.0.

CC BY NC SA: The CC BY-NC-SA license allows users to copy, to create extracts, abstracts and new works from the Article, to alter and revise the Article, provided this is not done for commercial purposes, and that the user gives appropriate credit (with a link to the formal publication through the relevant DOI), provides a link to the license, indicates if changes were made and the licensor is not represented as endorsing the use made of the work. Further, any new works must be made available on the same conditions. The full details of the license are available at http://creativecommons.org/licenses/by-nc-sa/4.0.

CC BY NC ND: The CC BY-NC-ND license allows users to copy and distribute the Article, provided this is not done for commercial purposes and further does not permit distribution of the Article if it is changed or edited in any way, and provided the user gives appropriate credit (with a link to the formal publication through the relevant DOI), provides a link to the license, and that the licensor is not represented as endorsing the use made of the work. The full details of the license are available at http://creativecommons.org/licenses/by-nc-nd/4.0. Any commercial reuse of Open Access articles published with a CC BY NC SA or CC BY NC ND license requires permission from Elsevier and will be subject to a fee.

Commercial reuse includes:

- Associating advertising with the full text of the Article

- Charging fees for document delivery or access

- Article aggregation

- Systematic distribution via e-mail lists or share buttons

Posting or linking by commercial companies for use by customers of those companies.

\section{Other Conditions:}

v1.9

Questions? customercare@copyright.com or +1-855-239-3415 (toll free in the US) or+1-978-646-2777.

Figure 1-2, 1-4, 1-5, 1-6, 1-8, 1-11 reproduced from open access articles These open access articles distributed under the Creative Commons Attribution License which permits unrestricted use, distribution, and reproduction in any medium, provided the original work is properly cited. (CC BY 4.0). 


\section{Conference Abstracts:}

Liang,S., Kinghorn,A.B., Prague,J.K., Dhillo,W.S., Cass, A.E.G., Li, R.H.W. \& Tanner,J.A. Automated electrochemical sensing system for monitoring of luteinising hormone pulsatility. Aptamers Oxford (2018)

Liang,S., Kinghorn,A.B., Dhillo,W.S., Cass, A.E.G. \& Tanner,J.A. Continuous flow electrochemical sensing system for monitoring luteinising hormone pulsatility. Oligos Oxford (2018)

Liang,S., Kinghorn,A.B., Dhillo,W.S., Cass, A.E.G. \& Tanner,J.A. Development of an aptamer-enable electrochemical sensing system for luteinising hormone pulsatility measurement. Aptamer in bordeaux (2016)

Liang,S., Dirkzwager,R.M. \& Tanner,J.A. Rapid Prototyping Aptamer-Enabled Malaria Diagnostics Using Three-Dimensional Printing. Aptamer Oxford (2016) 


\title{
Automated electrochemical sensing system for monitoring of luteinising hormone pulsatility
}

\author{
$\underline{\text { Shaolin Liang }}{ }^{1,2}$, Andrew Kinghorn ${ }^{1}$, Waljit Dhillo ${ }^{2}$, Anthony E G Cass ${ }^{3}$ and Julian A \\ Tanner ${ }^{1}$. \\ ${ }^{1}$ School of Biomedical Science, Faculty of Medicine, University of Hong Kong, 21 Sassoon \\ Road, Hong Kong SAR, P.R.China. \\ ${ }^{2}$ Department of Medicine, Imperial College London, South Kensington Campus, SW7 \\ $2 A Z$, UK \\ ${ }^{3}$ Department of Chemistry, Imperial College London, South Kensington Campus, SW7 \\ $2 A Z$, UK
}

Normal fertility in human involves a highly orchestrated signal communication crossing the axis of hypothalamic-pituitary-gonadal (HPG). The pulsatile release of Luteinizing Hormone (LH) from the pituitary gland is the key element in this "concerto" for the simulation of sex steroid hormones synthesis and the production of mature eggs. Specific alterations in LH pulsatile pattern are linked to hypothalamic dysfunction in female patient with anovulatory infertility - by knowing the information of this pattern, clinicians can decide whether the patient needs treatment with pituitary hormones to recover the normal ovulational function.

Here, we propose a novel platform using aptamer-based electrochemical sensor integrated with open-source liquid handling robot to assess LH pulsatility profile. A methylene blue (MB) modified LH specific aptamer was generated and adapted to a microwire electrode device designed for 96 well plate auto-handling. The sensor measures $\mathrm{LH}$ base on the structure switching upon binding associated with the electron transfer rate changes of the MB redox label.

To evaluate the performance of the system, LH pulsatility profile of Menopause female (high LH amplitude), normal female/male (normal LH amplitude) and female with hypothalamic amenorrhea (no LH pulsatility) was measured automatically. (total patient number $=12$, each has 50 blood samples collected every 10 mins in 8 hours). Our result shows the system can distinguish LH pulsatile pattern among the cohorts and pulsatility profiles were consistent with the result measured by clinical assays (same sample sets).

The automatic system shows a high potential on applying aptamer-based sensing clinically as it competes the current automated immunometric assays system with lower cost (reagent-less) and less complicated setup. It has the potential to be further developed as a POC diagnostic tool for the infertile patient caused by $\mathrm{LH}$ defect and assist clinicians to "personalised" treatment with hormonal therapy. 


\title{
Continuous flow electrochemical sensing system for monitoring luteinising hormone pulsatility
}

\author{
Shaolin Liang ${ }^{1}$, Waljit Dhillo ${ }^{2}$, Anthony E G Cass $^{3}$ and Julian A Tanner ${ }^{1}$. \\ ${ }^{1}$ School of Biomedical Science, Faculty of Medicine, University of Hong Kong, 21 Sassoon \\ Road, Hong Kong SAR, P.R.China. \\ ${ }^{2}$ Department of Medicine, Imperial College London, South Kensington Campus, SW7 \\ 2AZ, UK \\ ${ }^{2}$ Department of Chemistry, Imperial College London, South Kensington Campus, SW7 \\ 2AZ, UK
}

Normal fertility in human involves a highly orchestrated signal communication crossing the axis of hypothalamic-pituitary-gonadal (HPG). The pulsatile release of Luteinizing Hormone (LH) from pituitary gland is the key element in this "concerto" for the simulation of sex steroid hormones synthesis and the production of mature eggs. Specific alterations in LH pulsatile pattern are linked to hypothalamic dysfunction in female patient with anovulatory infertility - by knowing the information of this pattern, clinicians can decide whether the patient needs treatment with pituitary hormones to recover the normal ovulational function. Here, we propose a novel sensing platform using aptamer-based electrochemical sensor integrated with a customized continuous flow cell for measuring LH pulsatility. We generated a $\mathrm{LH}$ specific aptamer $(\mathrm{kd}=300 \mathrm{nM})$ with a relatively fast $\mathrm{k}_{\mathrm{on}}$ and $k_{\text {off }}$ rate $\left(K_{\text {on }}=2.15 \times 10^{3} \mathrm{M}^{-1} \mathrm{~s}^{-1}, \mathrm{k}_{\text {off }}=1.2 \times 10^{-3} \mathrm{~s}^{-1}\right)$. The $t_{1 / 2}$ of the dissociation stage is less than 5 mins, this feature could benefit the continuous detection of the sensor design (10 mins per data point is the gold standard for LH pulsatility measurement). Methylene blue (MB) modified aptamer was immobilised onto gold electrode and multiple measurements of LH samples with different concentration were achieved by recording the current changes via Square Wave Voltammetry. This system is able to monitor $\mathrm{LH}$ induced structural switching of the aptamer by determining electrochemical currents associated with the distance between the redox label and the electrode surface and has the potential to be further developed as a POC diagnostic tool for the infertile patient caused by LH defect and assist clinicians to "personalized" treatment with hormonal therapy. 


\title{
Development of aptamer-based electrochemical sensing system for monitoring luteinising hormone pulsatility
}

\author{
Shaolin Liang ${ }^{1}$, Waljit Dhillo², Anthony E G Cass $^{3}$ and Julian A Tanner ${ }^{1}$. \\ ${ }^{1}$ School of Biomedical Science, Faculty of Medicine, University of Hong Kong, 21 Sassoon \\ Road, Hong Kong SAR, P.R.China. \\ ${ }^{2}$ Department of Medicine, Imperial College London, South Kensington Campus, SW7 \\ $2 A Z, U K$ \\ ${ }^{2}$ Department of Chemistry, Imperial College London, South Kensington Campus, SW7 \\ 2AZ, UK
}

Normal fertility in human involves a highly orchestrated signal communication crossing the axis of hypothalamic-pituitary-gonadal (HPG). The pulsatile release of Luteinizing Hormone (LH) from pituitary gland is the key element in this "concerto" for the simulation of sex steroid hormones synthesis and the production of mature eggs. Specific alterations in LH pulsatile pattern are linked to hypothalamic dysfunction in female patient with anovulatory infertility - by knowing the information of this pattern, clinicians can decide whether the patient needs treatment with pituitary hormones to recover the normal ovulational function. However, there is currently no clinical feasible diagnostic tool available for the measurement of LH pulsatility, which majorly due to the large blood sample volume requirement and high sampling frequency. The single measurement by antibody-based assay only provides rough information about the $\mathrm{LH}$ and this data is not sufficient enough for the clinicians to decide treatment options. Here, we propose a novel diagnostic strategy by using an electrochemical aptamer-based point-of-care (POC) biosensing system to continuously monitor the $\mathrm{LH}$ concentration in the patient with infertility. We generated a $\mathrm{LH}$ specific aptamer $(\mathrm{Kd}=300 \mathrm{nM})$ with a relatively fast $\mathrm{k}_{\text {on }}$ and $k_{\text {off }}$ rate $\left(K_{\text {on }}=2.15 \times 10^{3} \mathrm{M}^{-1} \mathrm{~s}^{-1}, \mathrm{k}_{\text {off }}=1.2 \times 10^{-3} \mathrm{~s}^{-1}\right)$ according to our SPR results. The $\mathrm{t}_{1 / 2}$ of the dissociation stage is less than 5 mins, this feature could benefit the continuous detection of the sensor design (10 mins per data point is the gold standard for LH pulsatility measurement). We labelled this aptamer with ferrocene probe and adapted it to a MICRUX electrochemical system for further electrochemical characterization. This system is able to monitor LH induced structural switching of the aptamer by determining electrochemical currents associated with the distance between the redox label and the electrode surface and has the potential to be further developed as a POC diagnostic tool for the infertile patient caused by LH defect and assist clinicians to "personalized" treatment with hormonal therapy. 


\title{
Rapid Prototyping Aptamer-Enabled Malaria Diagnostics Using Three-Dimensional Printing
}

\author{
Shaolin Liang ${ }^{1}$, Roderick M Dirkzwager ${ }^{1}$ and Julian A Tanner ${ }^{1}$. \\ ${ }^{1}$ School of Biomedical Science, Faculty of Medicine, University of Hong Kong, 21 Sassoon \\ Road, Hong Kong SAR, P.R.China.
}

The integration of three-dimensional (3D) printing into diagnostic development gives the creative power for rapid prototyping diagnostic devices by adapting basic molecular technologies. Thanks for the quick printing time and low printing cost, customized parts can be generated to fit different assay formats and optimized via the process of "design test - redesign". In this study, we present the development of 3D printed aptamerenabled diagnostic devices based on our previously reported aptamer-tethered enzyme capture (APTEC) assay for malaria diagnosis. A paper-based syringe test and a magnetic bead-based well test were developed using 3D printed parts. Both were found to successfully detect the recombinant malaria biomarker Plasmodium falciparum lactate dehydrogenase ( $P f \mathrm{LDH}$ ) at $\mathrm{ng} \mathrm{mL}^{-1}$ concentrations using low sample volumes and function using purified or spiked whole blood samples. This study demonstrates the ability of translating a biochemical assay into workable diagnostic device prototypes by 3D printing rapid prototyping. This technology, intended for rapid diagnostic tests for malaria, is distinct and carries many potential advantages relative to established lateral flow immunochromatographic approaches.

shaolin2@hku.hk

rdirkz@hku.hk

jatanner@hku.hk 


\section{Research Publications:}

Tang,M.S.L., Shiu,S.C.C., Godonoga,M., Cheung,Y.W., Liang,S., Dirkzwager, R.M., Kinghorn,A.B., Fraser,L.A., Heddle,J.G. \& Tanner,J.A. An aptamer-enabled DNA nanobox for protein sensing. Nanomedicine (2018)

Kinghorn,A.B., Fraser,L.A., Liang,S., Shiu,S.C.C and Tanner,J.A. Aptamer Bioinformatics. IJMS (2017)

Fraser,L.A., Kinghorn,A.B., Dirkzwager, R.M., Cheung,Y.W., Liang,S., Lim,B., Shiu,S.C.C., Tang,M.S.L., Andrew,D., Manitta,J., Richards,J.S \& Tanner,J.A. A Portable Microfluidic Aptamer-Tethered Enzyme Capture (APTEC) biosensor for malaria diagnosis. Biosensors and Bioelectronics (2017)

Shiu,S.C.C., Cheung,Y.W., Dirkzwager, R.M., Liang,S., Kinghorn,A.B., Fraser,L.A., Tang,M.S.L., and Tanner,J.A. Aptamer-Mediated Protein Molecular Recognition Driving a DNA Tweezer Nanomachine. Advanced Biosystems (2017)

(Co-first author) Dirkzwager,R.M., Liang,S., \& Tanner,J.A. Development of aptamer-based point-of-care diagnostic device for malaria using 3D printing rapid prototyping. ACS Sensors (2016)

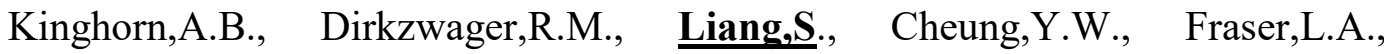
Tang,M.S.L \& Tanner,J.A. Aptamer Affinity Maturation by Resampling and Microarray Selection. Analytical Chemistry (2016)

Fraser, L.A., Kinghorn,A.B., Tang, M.S.L., Cheung,Y.W., Lim,B., Liang,S., Dirkzwager, R.M. \& Tanner,J.A. Oligonucleotide functionalized microbeads: indispensable tools for high-throughtput aptamer selection. Molecules (2015) 


\title{
Aptamer Bioinformatics
}

\author{
Andrew B. Kinghorn, Lewis A. Fraser (D), Shaolin Liang, Simon Chi-Chin Shiu (i) and \\ Julian A. Tanner* \\ School of Biomedical Sciences, Li Ka Shing Faculty of Medicine, The University of Hong Kong, \\ Pokfulam, Hong Kong SAR China; kinghorn@hku.hk (A.B.K.); lewis-fraser@hku.hk (L.A.F.); \\ shaolin2@hku.hk (S.L.); simon156@hku.hk (S.C.-C.S.) \\ * Correspondence: jatanner@hku.hk; Tel.: +852-3917-9472; Fax: +852-2855-1254
}

Received: 31 October 2017; Accepted: 20 November 2017; Published: 24 November 2017

\begin{abstract}
Aptamers are short nucleic acid sequences capable of specific, high-affinity molecular binding. They are isolated via SELEX (Systematic Evolution of Ligands by Exponential Enrichment), an evolutionary process that involves iterative rounds of selection and amplification before sequencing and aptamer characterization. As aptamers are genetic in nature, bioinformatic approaches have been used to improve both aptamers and their selection. This review will discuss the advancements made in several enclaves of aptamer bioinformatics, including simulation of aptamer selection, fragment-based aptamer design, patterning of libraries, identification of lead aptamers from high-throughput sequencing (HTS) data and in silico aptamer optimization.
\end{abstract}

Keywords: aptamer; simulation; in silico selection; molecular dynamics; fragment based design; HTS

\section{Introduction}

Aptamers are short nucleic acid sequences capable of specific, high-affinity molecular binding [1,2]. Aptamers are isolated via SELEX (Systematic Evolution of Ligands by Exponential Enrichment) (Figure 1), an evolutionary process in which successive rounds of selection and amplification are used to enrich an aptamer library for high affinity aptamers. Aptamers are among the simplest of genetic entities, having both genotypic and phenotypic properties and being capable of heredity in an in vitro selection experiment. Their combinatorial complexity poses many questions and problems that are well suited to computational analysis. Many computational approaches have been applied to aptamers, bringing together different disciplines and technologies. This review encompasses a broad range of aptamer bioinformatics approaches including simulation of aptamer selection, aptamer selection by molecular dynamics, patterning of libraries, identification of lead aptamers from high-throughput sequencing (HTS) data, and in silico aptamer optimization. We aim to describe and contrast these methods so that aptamer scientists might make use of the diverse array of bioinformatics resources available. 


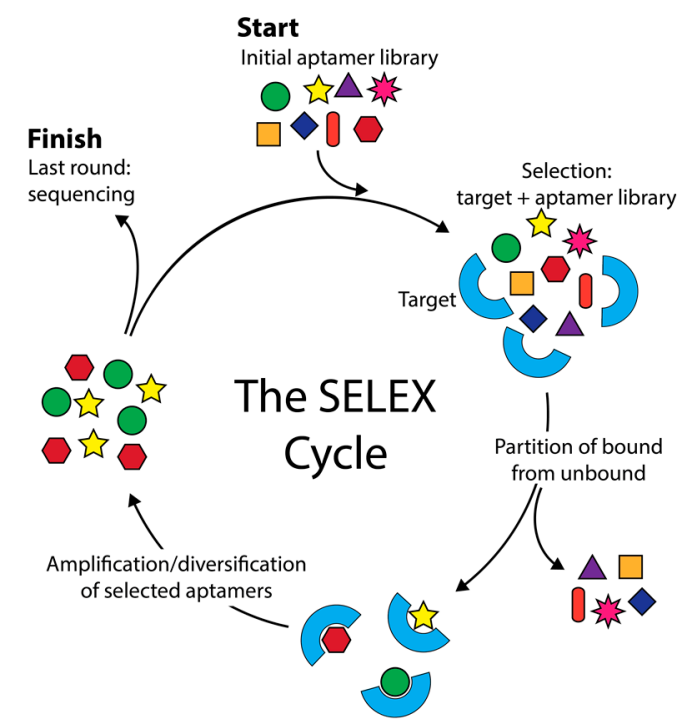

Fighure 1. The SELEX (Systhmatu்EE Sterrex

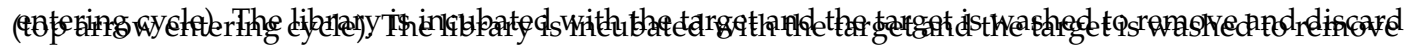

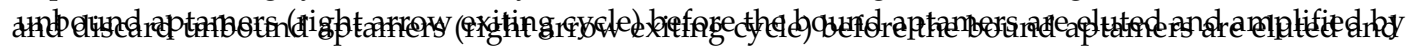

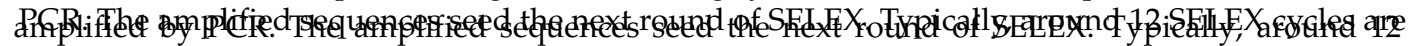

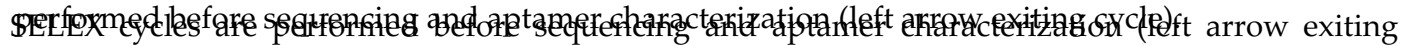
cycle).

2. Simulation of Aptamer Selection

2. Simulation of Aptamer Selection

Aptamer selection is complex. Complexity is found in both the myriad of experimental parameters

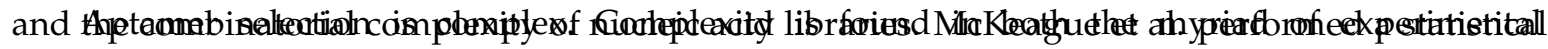

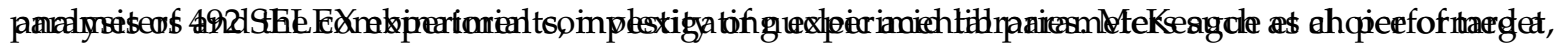

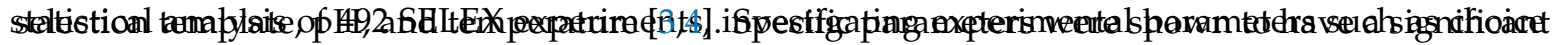

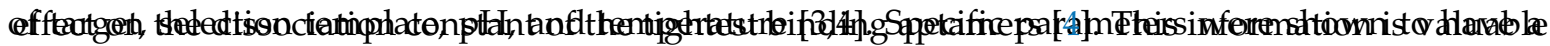

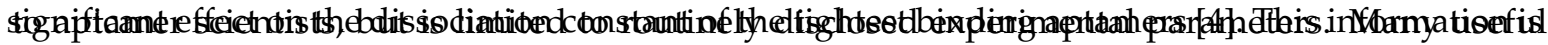

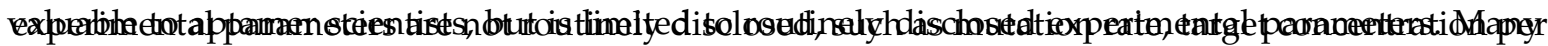

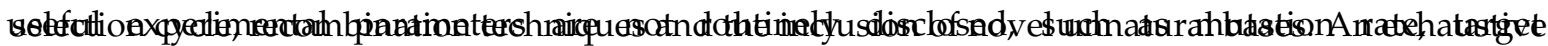

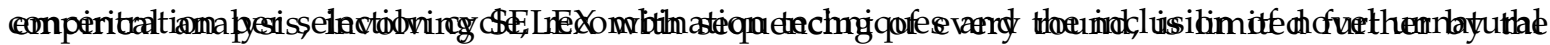

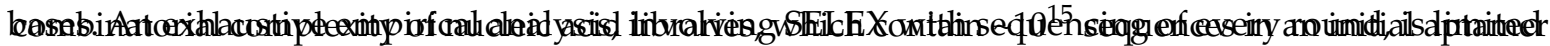

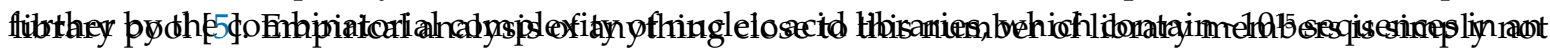

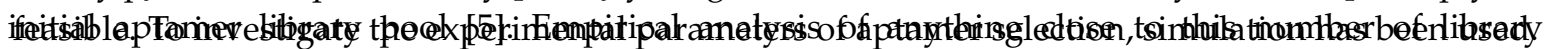

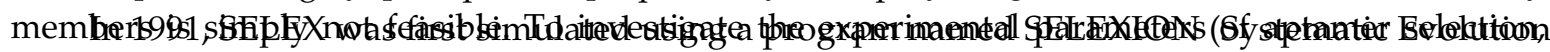

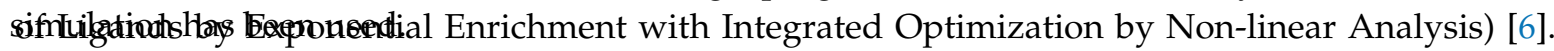

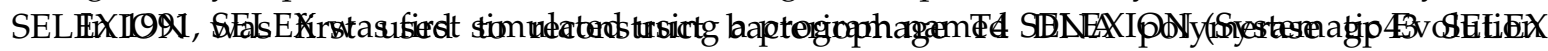

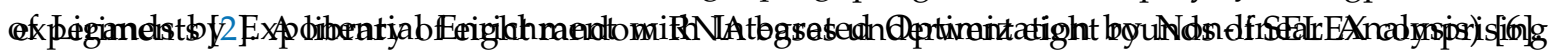

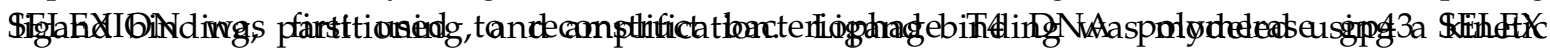

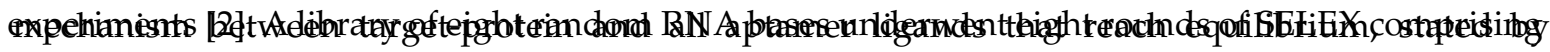
ligaind abiald[ágaspfottatiosing, and amplification. Ligand binding was modeled using a kinetic mechanism between target-protein and all aptamer ligands that reach equilibrium, stated by Irvine at al. [6] as follows:

$$
\begin{aligned}
& (P f)+\left(R N A f_{i}\right) \underset{\ll+i}{\longrightarrow}\left(P: R N A_{i}\right) \quad i=1, \ldots n, \\
& (\boldsymbol{P f})+\left(\boldsymbol{R} \boldsymbol{N} \boldsymbol{A} \boldsymbol{f}_{\boldsymbol{i}}\right) \stackrel{\substack{k-i \\
k}}{\longleftarrow}\left(\boldsymbol{P}: \boldsymbol{R} \boldsymbol{N} \boldsymbol{A}_{\boldsymbol{i}}\right) \quad \boldsymbol{i}=\mathbf{1}, \ldots \boldsymbol{n},
\end{aligned}
$$

where $(P f)$ was the free protein concentrat $(P: R N A)$ was the protein-RNA species $i$. complex concentration, $k+i$ was the rate constant for where $(P)$ ) was the rree protein concentration, $\left(R N A f_{i}\right)$ was the tree RNA species of $l$ concentration, $(P: R N A i)$ was the protein-RNA species $i$ complex concentration, $k+i$ was the rate constant for association of free protein and free RNA species $i, k-i$ is the rate constant for dissociation of proteinRNA species $i$ complexes, $(P: R N A i)$ was the protein-RNA species $i$ complex concentration, and $n$ is 
protein-RNA species $i$ complexes, $\left(P: R N A_{i}\right)$ was the protein-RNA species $i$ complex concentration, and $n$ is the number of RNA sequences with a unique set of rate constants [6]. Partitioning efficiency for the reconstruction was set to $80 \%$ of bound aptamers and $0.1 \%$ of unbound aptamers. Amplification of partitioned aptamers involved reverse transcription to CDNA and PCR amplification before library generation using transcription. The experimental parameters of the gp43 selection [2] were reconstructed and underwent simulation as a proof of principle for SELEXION [6]. These simulations indicated that the equilibrium mechanism proposed above for SELEX was sufficient to explain the high levels of enrichment after just a few rounds observed in the laboratory experiments. Following the reconstruction simulation of the gp43 selection, several properties were investigated using SELEXION including predicted enrichment under different conditions, optimal protein concentration when dissociation constant $\left(K_{\mathrm{D}}\right)$ estimates are known, near-optimum protein concentration with no estimate for $K_{\mathrm{D}}$, determination of sufficient protein concentration with no estimate for $K_{\mathrm{D}}$ or background, likelihood of SELEX success, and finally sequence representation in the random library pool [6]. SELEXION took a thorough approach to modeling ligand binding. However, a possible shortcoming would be the determination of aptamer properties such as $K_{\mathrm{D}}$. The binding affinities in terms of $K_{\mathrm{D}}$ were distributed without reference to aptamer sequence. For the reconstruction there were just five unique $K_{\mathrm{D}}$ values for all 65,536 unique aptamers in the initial library.

In 1998, Irvine et al.'s work [6] was extended and the program MultiSELEXION was coded to investigate SELEX against multiple targets [7]. MultiSELEXION allowed the investigation of problems arising from the use of contaminated protein preparations in SELEX, as well as analysis of complex target selections such as Cell-SELEX [8] and in vivo SELEX [9]. It was found that in most cases SELEX is capable of isolating differing ligands against the different targets in a heterogeneous mixture, irre SELEX spective of large variations in target concentrations or aptamer/target affinities. However, a low relative partitioning efficiency for a given target in a mixture gives a greatly reduced rate of selection of high-affinity aptamers [7].

Similarly to Irvine et al. [6] and Vant-Hull et al. [7], Chen et al. devised a SELEX simulation model that uses ligand binding based on equilibrium between target aptamer ligands and was applied to subtractive SELEX [10] as well as SELEX against a complex mixture [11]. This difference highlights and simulates selection pressures in SELEX experiments. Further similarities to Irvine et al. [6] and Vant-Hull et al. [7] included the binding affinities in terms of $K_{D}$ being distributed without reference to aptamer sequence. Chen et al. used just 10 unique $K_{\mathrm{D}}$ values for all aptamers in the simulations [10].

Wang et al. developed a model that focused on the two SELEX parameters, target concentration and the effect of nonspecific binding [12]. The model represented ligand binding using equilibrium kinetics similarly to Irvine et al. [6] and Vant-Hull et al. [7]. Partitioning was modeled in two ways: without background binding, which was intended to mimic microfluidic selection; and with background binding, which was intended to mimic nitrocellulose filter-based separation. Aptamer binding properties were normally distributed [12] as hypothesized in the literature [13,14]. Wang et al. [12] found that "without background binding" conditions, an increasing amount of target decreases the selection efficiency. Under "with background binding" conditions, there is an optimum target concentration that increases with increasing background binding. Interestingly, under multiple selection rounds and "with background binding" condition the optimum target concentration for achieving maximum enrichment increases with each SELEX round. This is contrary to the generally accepted practice of reducing the target concentration as SELEX progresses. The reason for this trend of increasing optimum target concentration in successive SELEX rounds could be the modeling of background binders. The more target, the greater the number of specifically binding aptamers make it to the next round, therefore the higher the ratio of specific to nonspecific binders and the higher the average $K_{\mathrm{D}}$ value. One aspect that SELEX Wang et al.'s model and many other models do not take into account is the possibility of adaptation in the aptamer pool giving rise to aptamers with increasingly tighter $K_{\mathrm{D}}$ values. 
Spill et al. developed a model that simulates Capture-SELEX and includes non-covalent ligand-substrate immobilization [15]. Aptamer-target binding was represented using a hybrid approach whereby an equilibrium constant is combined with a stochastic probability model. Following partitioning, the amplification of selected aptamers was simulated. Of particular interest is that the initial library $K_{\mathrm{D}}$ distribution has a dramatic effect on the outcome of the simulation. Additionally, the impact of distribution noise and the downstream effects on the total target concentration were assessed. The use of a stochastic model and Monte Carlo simulation highlighted sensitivity of SELEX to stochastic variation. Twenty very tight binders are capable of outcompeting $10^{15}$ library members or can be totally lost.

Simulation of SELEX has given insight into how an aptamer scientist might optimize the SELEX protocol. SELEX has both vast complexity in terms of the number of sequences (typically around $10^{15}$ ), and informational complexity associated with each individual aptamer's sequence, folding, and target binding. The aforementioned simulations have focused on representing the vast complexity of SELEX and neglected the informational complexity of individual aptamer sequences. All binding properties of aptamers are selected randomly or from a distribution with no relevance to the aptamer sequence. For particular questions about SELEX, including the role of adaptation and the occurrence of divergent and convergent evolution, a more thorough binding model is required.

Hoinka et al. coded a program to simulate the aptamer selection process called "AptaSim" [16]. AptaSim aimed at realistically recreating the selection process during SELEX with the intention of investigating the effect of error-prone PCR on aptamer selection. An initial library pool was generated using a first-order Markov Model, previously trained on early SELEX round selection data. The generated aptamer was randomly assigned a copy number and binding affinity within a predefined range. Iterative cycles of capture and amplification were then simulated where the capture probability is related to an aptamer's copy number and binding affinity, and amplification is subject to a specified probability of mutation. The binding model used attributed aptamer affinities at random without relevance of sequence. Additionally, mutated versions of these aptamers retained the original's attributed binding affinity. While AptaSim was an important step forward in simulating selection, enrichment and mutation copy number, AptaSim did not appropriately represent heritability or represent binding affinities correlated between related sequences, which is required for the study of SELEX as a genetic system.

Oh et al. used a string matching function as a binding model to simulate aptamer selection [17]. All aptamers were given a target binding score based on their similarity to a given "optimal aptamer" sequence [17]. This model does include heritability and binding correlation between related sequences. As string matching is not computationally demanding, this approach can be used for very large library sizes, which is more representative of aptamer selection. The drawback of string matching is that only close-range epistasis is possible and by using a one "optimal aptamer" model, the landscape is cone-shaped and would not represent a true aptamer binding landscape.

Wedge et al. used Kauffman's NK model [18] to represent ligand-target binding for the simulation of protein-directed evolution [19], a similar field to aptamer selection. The NK model is a robust mathematical model that serves as an objective function relating genotypic sequences to phenotypic fitnesses that make up a fitness landscape. Using the NK model, strings of informational digits of length $N$ are attributed fitness values equal to the sum of each digits interaction with $K$ other digits. In this way, epistatic and pleiotropic interactions can be modeled. The NK model has been used to describe many complex systems such as immunology [20], evolutionary biology [21], and economics [22]. The $N K$ model has also been related to aptamers [23]. In Wedge et al.'s [23] work the ligand properties were determined using an $N K$ model in which binary strings of length $N=100$ were used with random epistatic interactions varying from $K=0$ to 10 . The initial library size was 40,000 and during each of the 10 selection rounds, 1 to 4000 of the tightest binding ligands were partitioned. Varying degrees of selection pressure (number of ligands selected each round), mutation rate, and crossover (recombination) were tested and it was found that optimal directed evolution (DE) parameters were 
strong selection pressure, a high mutation rate, and that crossover is only valuable when epistasis is low to moderate $(K<5)$. While these results are valuable to the field of protein-directed evolution, the simulation did not mimic properties specific to SELEX.

The NK model can effectively represent the target binding of polymeric ligands such as proteins and aptamers. Besides the challenges for biological accuracy in representing base interactions within an aptamer, the classical NK model may have limitations in representing some aspects of biological

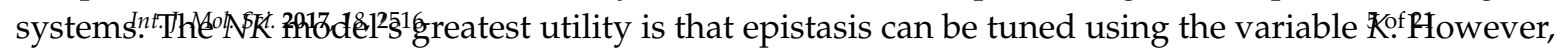
this epistasis is reasonably uniform throughout the sequence. To represent some biological systems,

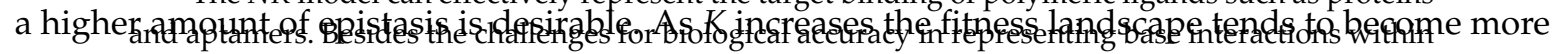

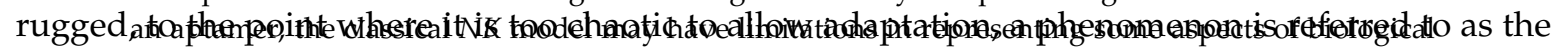

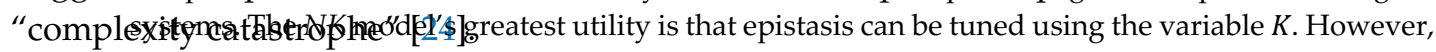

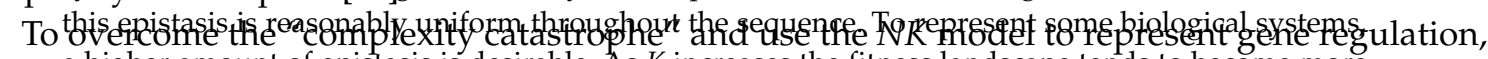

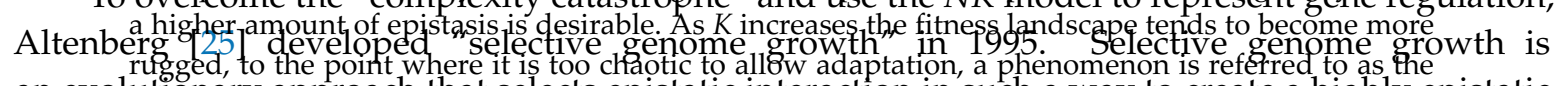

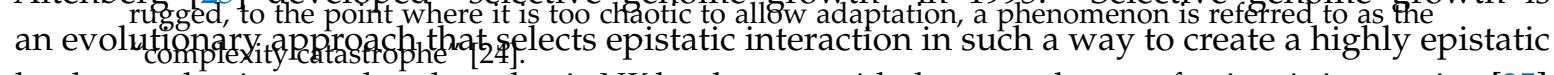

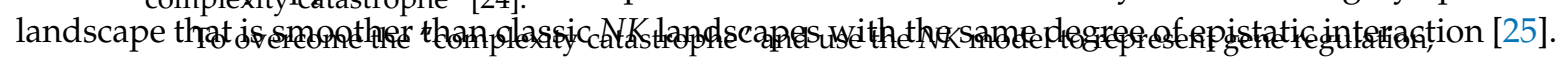

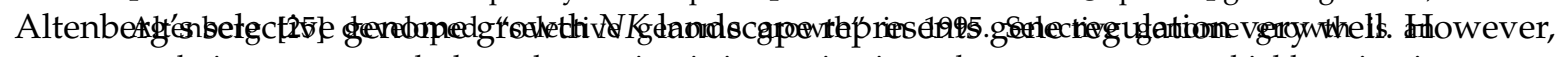

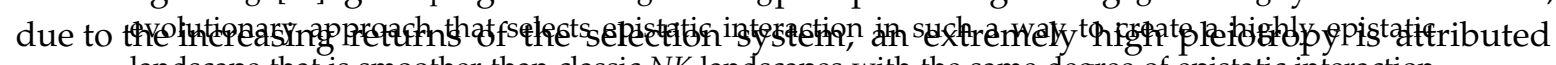

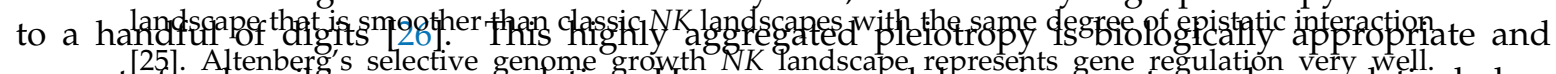

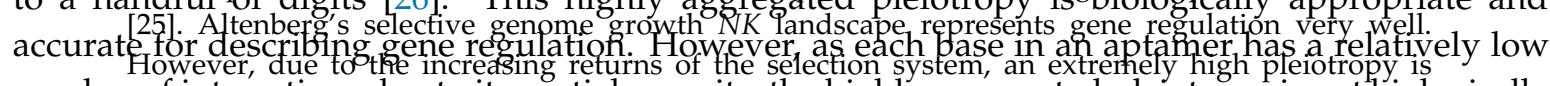

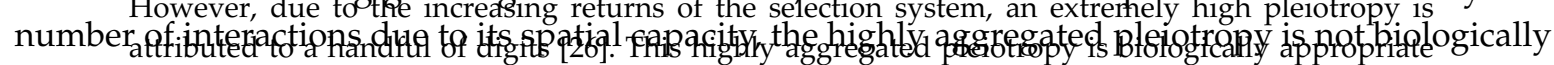

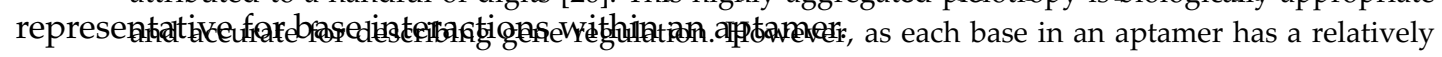

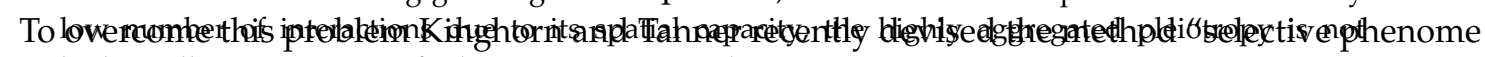

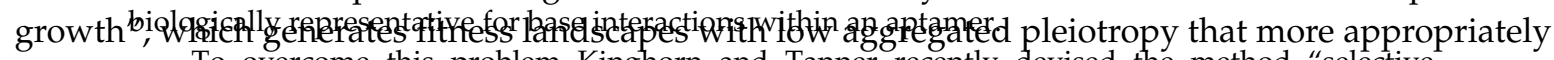

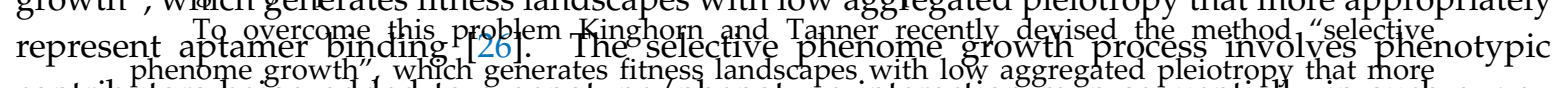

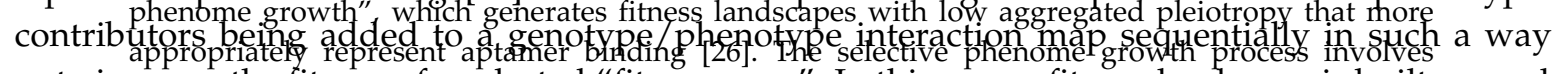

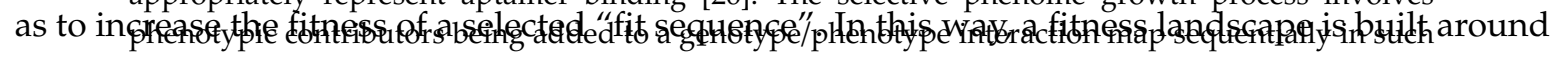

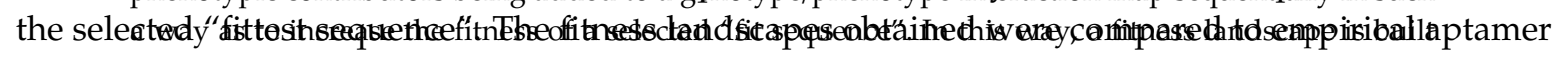

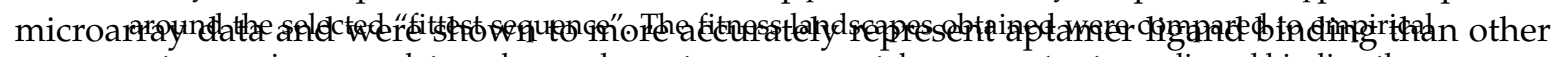

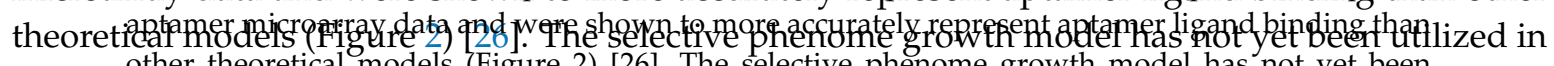

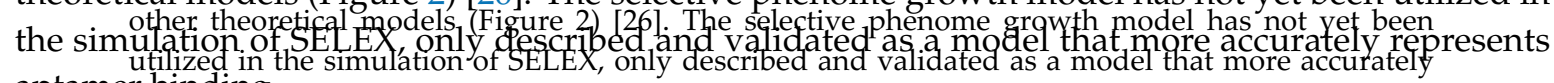
utilized in the simulation of
aptamerepindingentgaptamer binding.

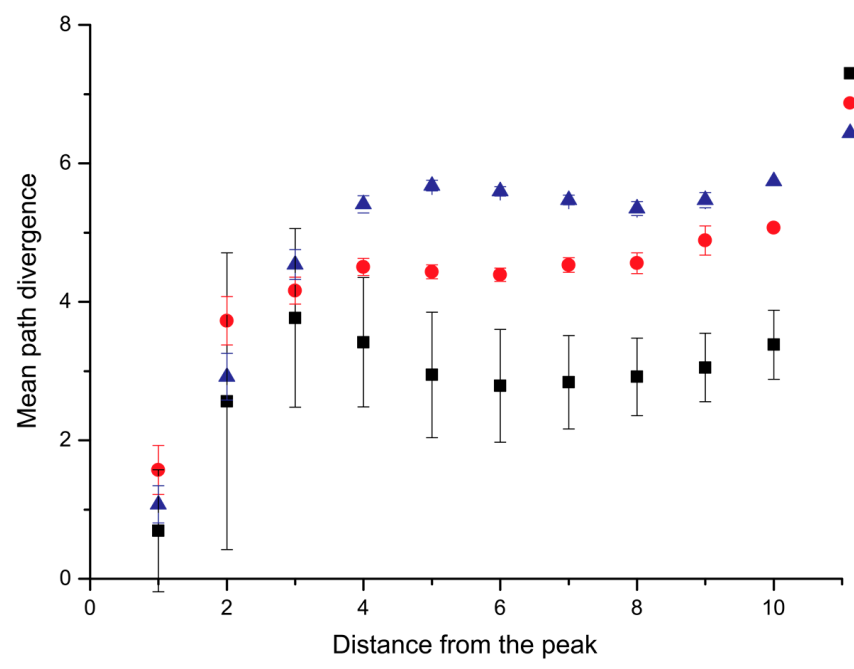

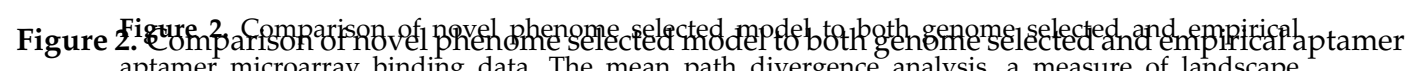

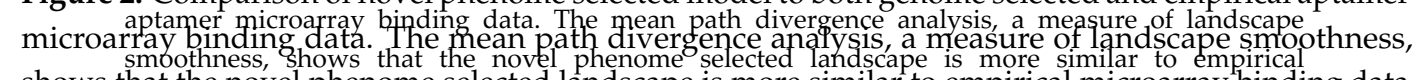

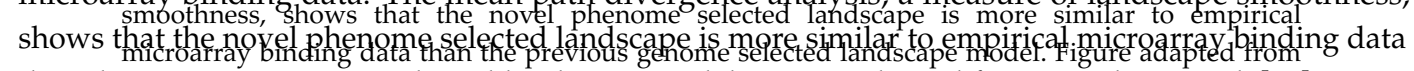
than therpordigute gengome selected landscape model. Figure adapted from Kinghorn et al. [26]. 
The no-free-lunch theorem states that all search algorithms perform exactly the same when averaged over all possible problems [27]. This infers that any elevated performance in one class of problem is exactly paid for in the performance of another class of problem. If there is discrepancy between a real-life system and a model used to describe it, for example an empirical SELEX experiment and a SELEX simulation, any elevated performance insight found using the simulation is exactly paid for in the performance of the real-life system. This illustrates the need for the simulation model to be as accurate as possible; otherwise optimizations will not translate to empirical SELEX experiments. The area least accurately modeled in SELEX simulations has been the aptamer binding model.

\section{Aptamer Selection by Molecular Dynamics}

Molecular dynamics have applications across biotechnology, including but not limited to protein studies, membrane transport, and drug discovery [28-31]. One particular application is to improve the efficacy of aptamer selections by computationally solving the three-dimensional structures of nucleic acids (NAs) and their targets, and simulating the physical forces involved in NA docking to a target. This is achieved by various N-body simulations that calculate the dynamic forces of the atoms and molecules of a NA within a binding site, in the form of a docking score. Docking scores can be used to identify sequences that bind to a target, defining a novel approach for aptamer discovery. Here, we will discuss studies wherein molecular dynamics has been used to enrich selection pools, optimize existing aptamers, and discover new aptamers.

\subsection{Whole Aptamer Docking}

For the purpose of this review we have divided in silico techniques into two categories: those that simulate the molecular dynamics of a whole aptamer and its target, and those that fragment an aptamer into discrete units to simulate binding interactions. We will discuss the literature that underpins in silico selections for whole aptamers.

Computationally predicting secondary and tertiary structures of NAs and targets reveals the steric and energetic properties of each structure. These predictions allow researchers to modify their selection pools to have a broader range of three-dimensional structures and NAs with more favorable free energy [32-34], and provides essential information for molecular docking simulations [35]. Many protein-NA structures have been solved experimentally using NMR and X-ray crystallography, for which there are large but limited libraries in the Protein Data Bank (PDB). If the structure has not been solved experimentally, homology modeling webserver services exist for both proteins and NAs [36-39].

In simulating the docking between a target and an aptamer, several non-covalent interactions are assessed including ionic interactions, hydrogen bonds, van der Waal's forces, hydrophobic interactions, base stacking interactions, and shape complementarity [35] (Figure 3A). Algorithms calculate the potential energy between interacting atomic components, known as force fields. For biological systems, the most frequently used MD force field simulations are CHARMM and AMBER [35]. Homology and modeling software for DNA-protein interactions is currently limited [35], as much of the software is based in analyzing protein-protein interactions. A coarse-grained force field has shown how dsDNA interacts with protein structures. Specific interactions are useful but limited in their scope and shape complementarity and internal DNA energy play an important role in simulating protein-DNA docking [40] (Figure 3B).

An initial attempt at in silico selection was proposed by Chushak and Stone. Computationally, they decreased RNA sequence search space in a selection pool by up to five orders of magnitude to enable conjugation of an enriched RNA selection pool to a microarray to improve high-throughput aptamer selections [34]. A three-step enrichment approach was used:

(1) Selection based on secondary structure - a set of criteria were used to identify and eliminate sequences with common simple structural motifs and high-energy unstable RNA sequences, both of which would be unlikely to form aptamers. 
(2) Selecting for conformational flexibility-a single RNA sequence can have a large range of three-dimensional conformations; the Rosetta RNA package [41] was used to generate these structures. Then the five lowest energy three-dimensional structures, and therefore those with the greatest conformational flexibility, were selected using the AMBER force field simulation and the generalized Born solvation model [42].

(3) Screening the RNA library with computational docking-a modified docking tool called DOVIS using Autodock v4 [43] was used to simulate interactions between all the generated RNA three-dimensional structures and small molecule targets. Docking was scored based on their calculated affinity for the targets. By selecting for the highest scoring sequences they effectively lowered the RNA pool size from $\sim 2.5 \times 10^{8}$ to $5 \times 10^{3}$. Six known aptamer-ligand complexes were used to validate this approach. Native aptamers were found in within the top $5 \%$ of in silico selected structures.

Confirming that molecular dynamic calculations align with experimental evidence provides further evidence that in silico approaches can complement aptamer selections. A software package that uses the CHARMM force field to analyze protein-protein interactions called Discovery Studio uses a docking simulation algorithm called ZDOCK $[35,44]$. ZDOCK was found to work effectively with short RNA-protein interactions [45] but was found to be ineffective when simulating longer RNA strands $[46,47]$. When combined with ZRANK, an algorithm that takes into account a range of attractive and repulsive forces, van der Waal's forces, and desolvation, effective simulation of protein-long-strand RNA was achieved $[35,48]$. Having confirmed the efficacy of this software package in conjunction with aptamer-protein interactions, Chen's research group mutated aptamers of angiopoiten-2 protein (Ang-2), a protein that regulates angiogenesis and is linked with the development and spread of cancer $[49,50]$. From the mutated strands, they selected three with high scores and tested them experimentally for binding with surface plasmon resonance (SPR). Based on binding affinity and SPR response, they claim one of these novel aptamers (Seq15_12_35, $K_{\mathrm{D}} 0.61$ ) has improved binding when compared to a high-affinity Ang-2 aptamer (Seq1, $K_{\mathrm{D}} 1.39$ ) found in the literature [48].

Selection can be a lengthy and costly process [51], especially when targeting human proteins for which native proteins may be expensive or commercially unavailable [52]. To lower the cost of selection, it is common to select an aptamer towards a recombinant or non-human version of the equivalent human protein [53]. There is an increased risk that the difference in homology between the native protein and the recombinant/non-human protein will result in selecting for an aptamer that will not bind to the native protein. This is the case for immune-checkpoint blockade receptor TIM3 [54], for which aptamers selected for murine binding aptamers lacked cross-reactivity with the human form. Based on the murine aptamer, Rabal et al. used a three-step bioinformatics process similar to those already discussed, but coupled cluster analysis with their chosen 3D docking algorithm, 3DRPC [55]. Clustering algorithm GROMACS [56] revealed highly populated clusters focused around specific binding sites. In four out of five cases, combining clustering with docking simulation revealed a binding mode and site that were not identified by docking simulation alone. They were able to show the scope of in silico aptamer-protein analysis by identifying a plausible binding site on murine TIM3 and aptamer binding mode that explains the lack of cross-reactivity in murine over human TIM-3 [54].

\subsection{Fragment-Based Aptamer Design and Docking}

Whole aptamer selections require massive computational resources and three-dimensional structures of both nucleotides and target. A fragment-based approach has been argued to simplify the process of in silico aptamer generation [57]. Tseng et al. presented a three-step approach in which they only require structural information of the target, known as the entropic fragment-based approach (EFBA) [57]. They first determined the probability distribution of the first nucleotide binding to the target. They then sequentially added nucleotides to the first, taking into account the probability distributions of the added nucleotide to its neighbors and the target. Finally, they determined a cutoff length based on an entropic criterion (information theory entropy). Once the target-NA complex was saturated and the interactions of the complex were at a global minimum irrespective of nucleotide 
target-NA complex was saturated and the interactions of the complex were at a global minimum

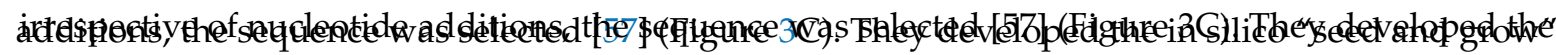

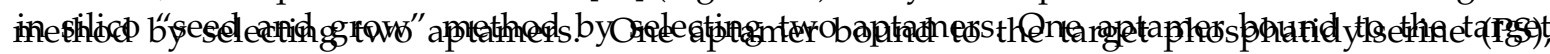

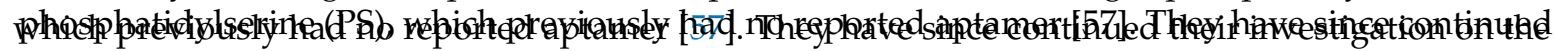

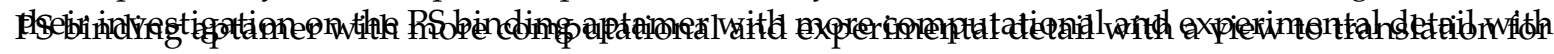
praieteatioutsen

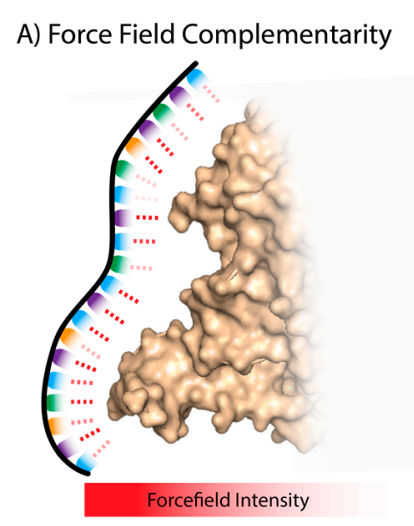

C) Seed and Grow Approach

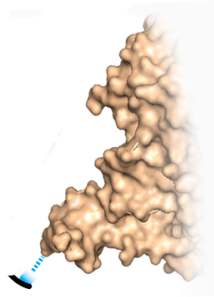

B) Shape Complementarity
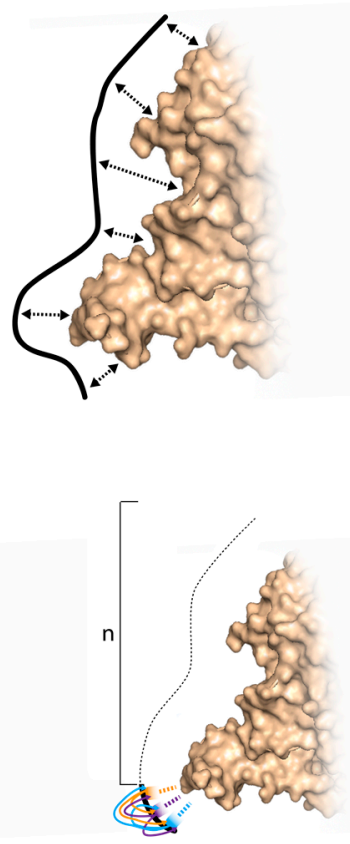

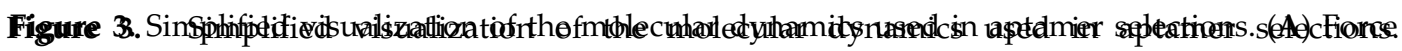

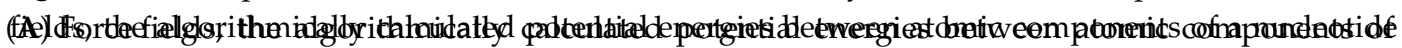

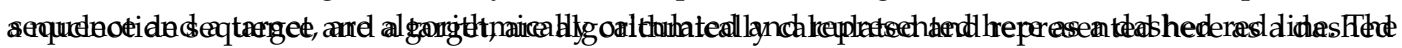

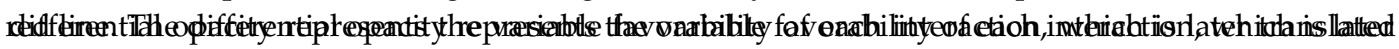

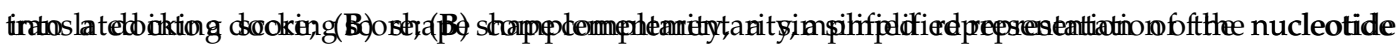

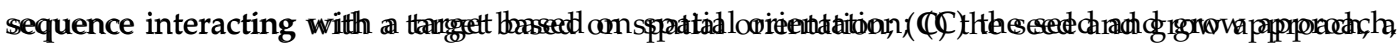

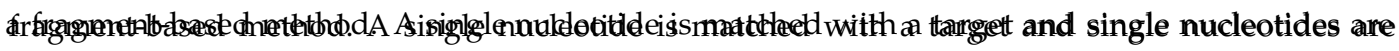

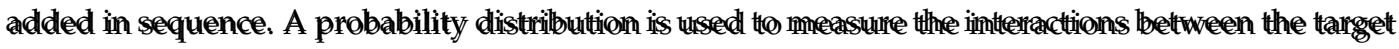

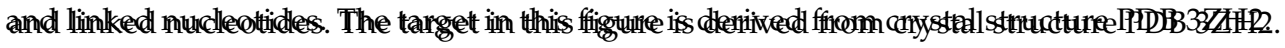

\section{Patterning of Libraries}

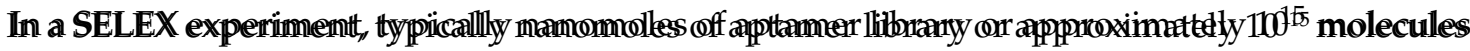

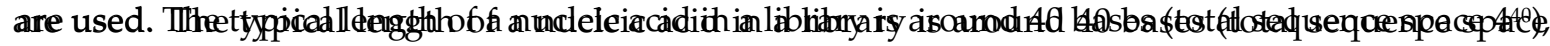

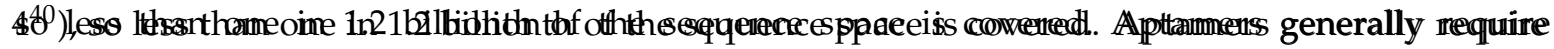
secondary structure to bind their targets, therefore increasing the occurrence of secomdary struicture in the library should enhance the success rate when selecting for an aptamer. Here, we wrill outtline

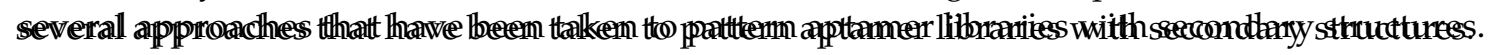

RNA aptamer and ribozyme selection analysis has shown that the presence of dlisttimetive

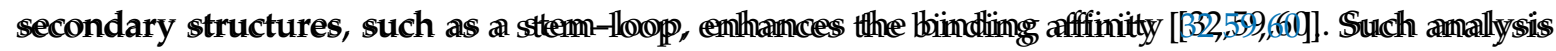

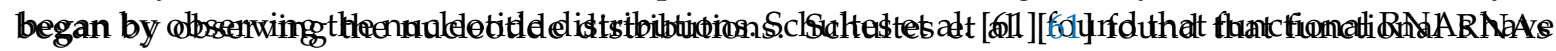

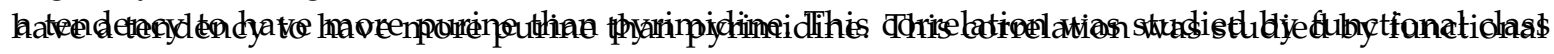

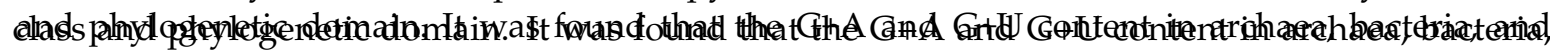

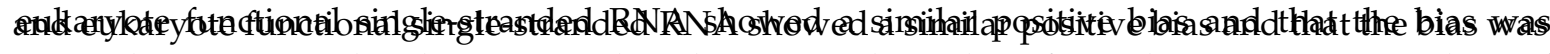

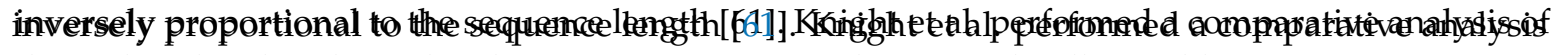

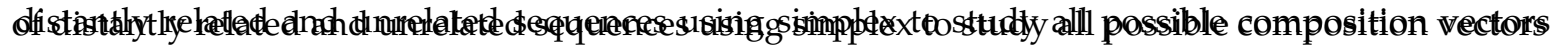
$(\mathrm{G}+\mathrm{A}, \mathrm{G}+\mathrm{U}$ and $\mathrm{G}+\mathrm{C})$ of isoleucine aptamer and hammerhead ribozyme [62]. A library size of $6.23 \times$ 
$(\mathrm{G}+\mathrm{A}, \mathrm{G}+\mathrm{U}$ and $\mathrm{G}+\mathrm{C})$ of isoleucine aptamer and hammerhead ribozyme [62]. A library size of $6.23 \times 10^{9}$ sequences containing $25 \% \mathrm{U}, 15 \% \mathrm{C}, 20 \% \mathrm{~A}$, and $40 \%$ G could maximize the probability of identifying both motifs (99\%). This study demonstrated that adjustment of base composition could be used to lower the total number of candidates in a SELEX (Systematic Evolution of Ligands by Exponential Enrichment) experiment.

Computational methods use sequence information to pattern initial libraries, which results in the evolution of more complex structures. For DNA aptamer selection, Ruff et al. patterned their initial library pool with alternating purine and pyrimidine, which was found to increase the formation of stem-loop structures that bind to streptavidin, immunoglobulin E (IgE), and vascular endothelial growth factor (VEGF) [63]. By sequencing pools from each selection round, they compared the selection efficiency for both random and patterned libraries. The patterned library was significantly enriched relative to the unpatterned library at the 10th round. For IgE, after adding restriction endonuclease to digest the tagged sequences, it was found that the enrichment was further significantly increased. The use of a patterned library in SELEX was able to select specific binders for all three molecules with affinity at nanomolar levels better than those selected from random libraries (streptavidin: $K_{\mathrm{D}}=105 \mathrm{nM}$, IgE: $K_{\mathrm{D}}=26 \mathrm{nM}$, VEGF: $K_{\mathrm{D}}=45 \mathrm{nM}$ ). These results showed that the use of a patterned library could increase the proportion of active aptamer, speed of selection, and affinity of the resultant aptamers [63].

This alternating purine and pyrimidine patterning strategy was enhanced when Martin et al. used a novel computational method to increase the structural complexity of a DNA library (Table 1) [64]. This patterning method reduced the size of the library, allowing the integration of the entire library onto a microarray, for the identification of a thrombin binding aptamer. The initial library was designed with UNAFold software using three major constraints. First, the first nucleotide of the aptamer must pair with the final one. Second, the number of unpaired bases must fluctuate between 10 and 30 for the 50-nucleotide strands. Third, there must be at least two stretches of unpaired nucleotides. This limited the total number of candidates in the library to 50,000 sequences. The selection results showed that the first six to eight bases of the top 15 sequences resembled thrombin binding aptamer and binding was also specific to thrombin. This demonstrated the effectiveness of using a patterned library on a microarray to select for aptamers.

Table 1. Library design used by Martin et al. [64].

\begin{tabular}{cc}
\hline Pattern & Library Design \\
\hline 1 & $(R Y)_{3}-\mathrm{N}_{4}-(\mathrm{RY})_{4}-\mathrm{N}_{3}-(\mathrm{RY})_{4}-\mathrm{N}_{4}-(\mathrm{RY})_{4}-\mathrm{N}_{3}-(\mathrm{RY})_{3}$ \\
2 & $(\mathrm{RRYY})_{2}-\mathrm{N}_{4}-(\mathrm{RRYY})-\mathrm{N}_{3}-(\mathrm{RRYY})-\mathrm{N}_{4}-(\mathrm{RRYY})-\mathrm{N}_{3}-(\mathrm{RRYY})-\mathrm{N}_{4}-(\mathrm{RRYY})_{2}$ \\
3 & $(\mathrm{RRYY})_{2}-\mathrm{N}_{4}-(\mathrm{RRRYY})-\mathrm{N}_{4}-(\mathrm{RR} R Y)-\mathrm{N}_{4}-(\mathrm{RRRY} Y)-\mathrm{N}_{4}-(\mathrm{RRYY})_{2}$ \\
4 & $(\mathrm{RRYY})_{2}-\mathrm{N}_{4}-(\mathrm{RY})_{3}-\mathrm{N}_{4}-(\mathrm{RY})_{3}-\mathrm{N}_{4}-(\mathrm{RY})_{3}-\mathrm{N}_{4}-(\mathrm{RRYY})_{2}$ \\
\hline
\end{tabular}

\footnotetext{
${ }^{1}$ Library designs of different patterns of alternating purine and pyrimidine. Pattern 1 library theoretically has $1.8 \times 10^{19}$ sequences and Pattern 2 has $3 \times 10^{20}$. Pattern 3 has three consecutive purines or pyrimidines, which may allow the formation of quadruplex, while Pattern 4 only allows alternating purines and pyrimidines. $R$ is purine, $\mathrm{Y}$ is pyrimidine, and $\mathrm{N}$ is a random mixture of purine and pyrimidine. Table adapted from [64].
}

The 1963 discovery of Hoogsteen base-pairing explains the formation of triplex and quadruplex structures $[65,66]$. The G-quadruplex structure now holds significant interest due to applications in therapeutics $[67,68]$ and diagnosis $[69,70]$. A G-quadruplex usually consists of four guanine tracks and a few tetrads [71]. The structural features include a broad surface of $\pi$-orbitals above and below the quadruplex that allow hydrophobic binding to targets such as nucleolin [72], hemin [73,74], and light-up fluorogens [75-77].

The binding capacity of G-quadruplex structures was exploited by McManus and Li, who integrated patterning into DNA libraries to select aptamers with quadruplex structures. They patterned the library with the following methods: inclusion of four G-tracts in the library while leaving the rest to be random; simplifying the complicated three-layer G-quadruplex into 


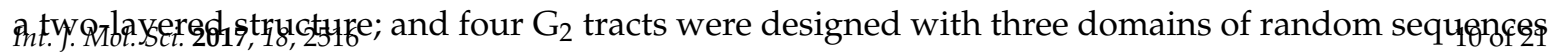
for loop formation $[71,78]$. The authors first investigated the effective loop length for the folding

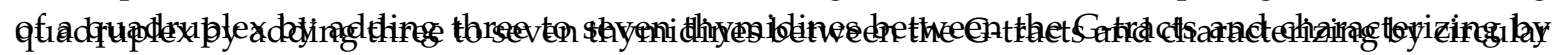

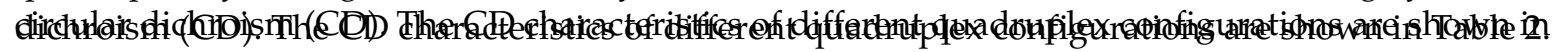

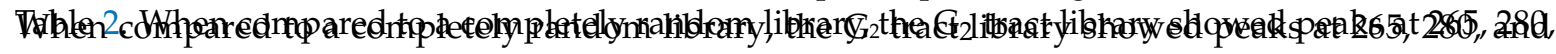

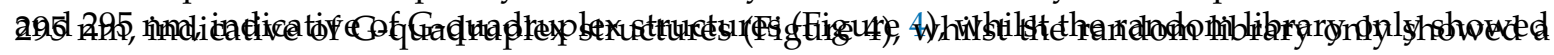

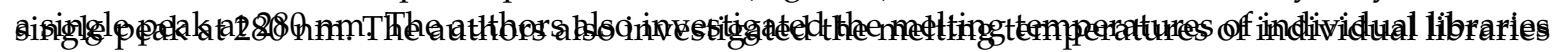

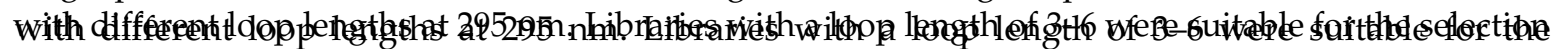

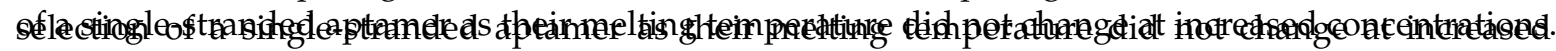

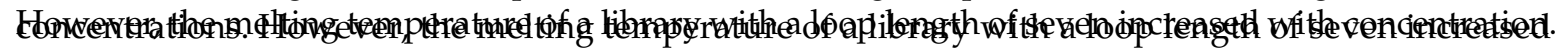

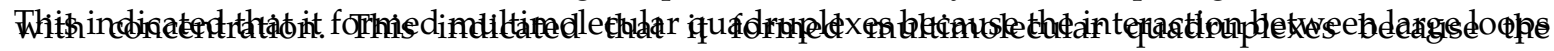

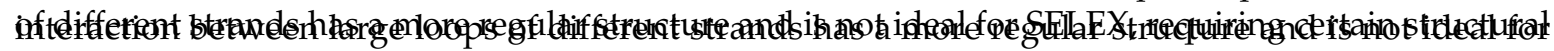

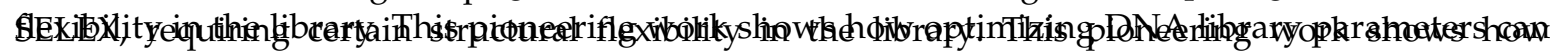

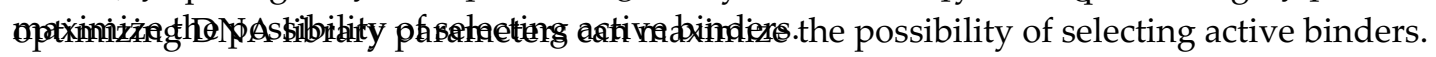

A

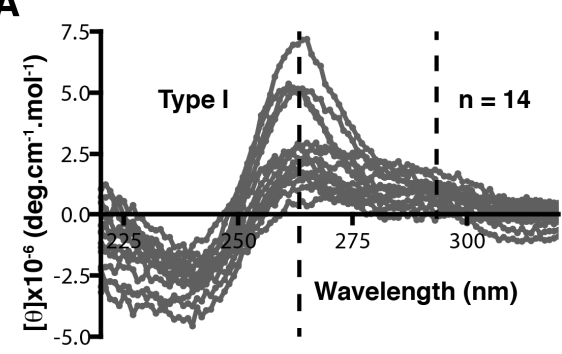

C

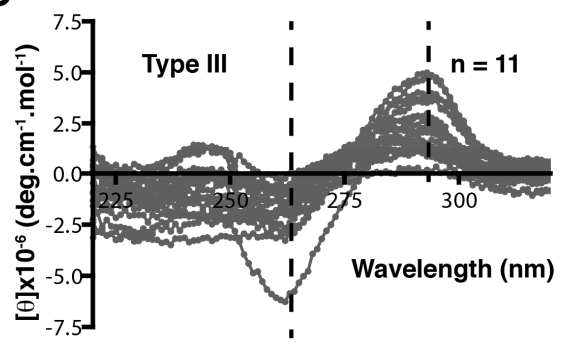

B

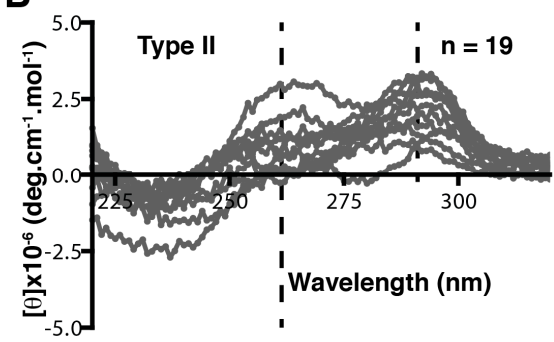

D

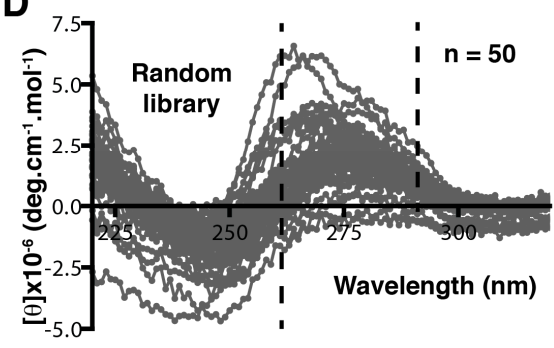

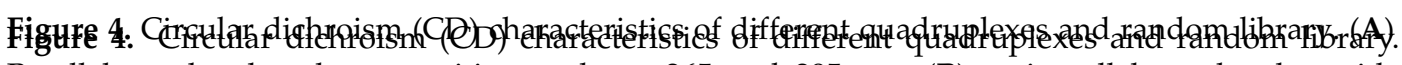

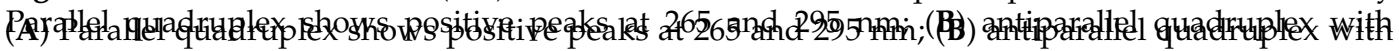
glyeosidic bond angles of the same orientation shows bimodal spectra as pesitive peaks at z6ry nm

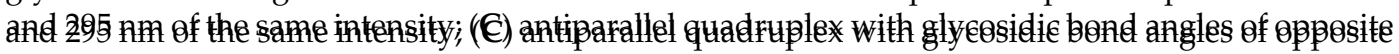

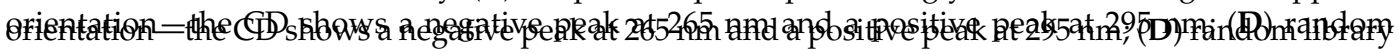
bilks

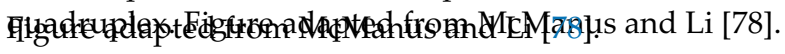

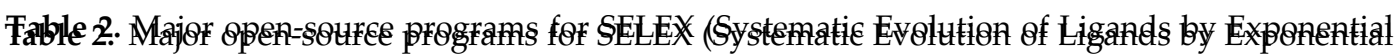

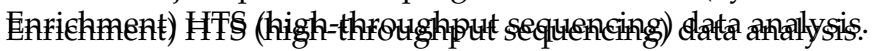

\begin{tabular}{|c|c|c|c|c|}
\hline Piroggan & $\begin{array}{c}\text { Operation } \\
\text { Operation System } \\
\text { System }\end{array}$ & Larlgaiagarage & CGist6tingingeklothod & $\begin{array}{l}\text { Validation } \\
\text { Validation Experiment } \\
\text { Experiment }\end{array}$ \\
\hline FASTAptamer & Mac/Linux & Perl & Levenshtein distance & HIV-1 Reverse Transcriptase \\
\hline AptFASTSA & Mac/MiagdXipex & $\mathrm{Java}^{\text {Perl }}$ & LSHekenshtieingdistance & Tränscriptase \\
\hline AptaCAtsterYAptaGUI & Maimy\&inux/PC & Pythghva & Struqtgramatiff-kasededclustering g & 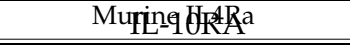 \\
\hline $\begin{array}{c}\text { AptaTrace } \\
\text { APTANI } \\
\text { PATTERNITY-seq }\end{array}$ & $\begin{array}{c}\text { Mac/Linux/PC } \\
\text { Linux } \\
\text { No details }\end{array}$ & $\begin{array}{l}\mathrm{C}++ \text {, Java } \\
\text { Python } \\
\text { No details }\end{array}$ & 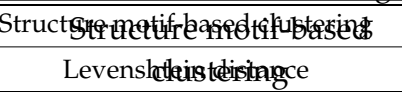 & $\begin{array}{c}\text { C-C chemokine receptor } \\
\text { Murine ILARa } \\
\text { Annexin-A2 }\end{array}$ \\
\hline AptaTrace & Mac/Linux/PC & $\mathrm{C}++$, Java & $\begin{array}{c}\text { Structure motif-based } \\
\text { clustering }\end{array}$ & $\begin{array}{c}\mathrm{C}-\mathrm{C} \text { chemokine } \\
\text { receptor }\end{array}$ \\
\hline PATTERNITY-seq & No details & $\begin{array}{c}\text { No } \\
\text { details }\end{array}$ & Levenshtein distance & Annexin-A2 \\
\hline
\end{tabular}


In a SELEX experiment, the random library provides low sequence space coverage and low structure space representation. Using stem-loop structures and/or patterned libraries can increase sequence space coverage and improve space representation. Although it is difficult to pinpoint the best method to pattern a library, it is clear that patterned libraries can enhance aptamer selection by reducing the time taken to select an aptamer, increasing the success rate of a selection, and improving the binding affinity of isolated aptamers [63].

\section{In Silico Aptamer Identification from High-Throughput Sequencing (HTS) Data}

\subsection{The Trend of Using HTS for Improving SELEX}

Initially developed for the purpose of tackling the increasing complexity of whole genome sequencing, HTS technologies have continued to evolve and change the landscape in many fields of biomedical research over the last 10 years [79]. Since the development of the first commercialized 454 sequencer, companies such as Illumina, Ion Torrent, and Oxford Nanopore technologies are all increasing sequencer capacity and reducing cost [80]. In the area of aptamer research, deep, high-throughput, and in-parallel DNA sequencing technologies allow the analysis of millions of sequences found in each round of aptamer selection, and thus open a new avenue for identification and optimization of aptamers [81]. HTS data obtained from each round of the selection can not only be used to monitor the dynamic sequence change of aptamer selection to identify the best-performing sequences in early rounds [82-84], but also as a tool for aptamer scientists to further investigate the enrichment principles of SELEX process such as selection efficiency [85], aptamer-target interactions [86], and mutation landscape [16].

Some of the earliest works applying HTS for identification of aptamers were performed by Schroeder's group in 2010. In the study, they applied a genomic RNA library-overlapping sequences constructed via PCR from the E. coli genome-to select against an RNA binding protein named Hfq. 454 sequencing was used to obtain sequence data for two of the last round libraries from the genomic selection and, for comparison purposes, the rounds of another selection that omitted the target binding step, to monitor the amplification variants of the genomic SELEX. By analyzing the HTS data, they successfully identified genomic RNA aptamers and discovered that these aptamers are predominant in the antisense transcripts $[87,88]$. In the same year, Soh's group pioneered DNA aptamer quantitative selection by applying microfluidic and HTS technologies. They performed three rounds of microfluidic device-assisted selection against platelet-derived growth factor BB (PDGF-BB) and sequenced each round of the selection via high-throughput sequencing. More than $1.7 \times 10^{7}$ sequences from each round of selection were obtained and the enrichment trajectory across different rounds was tracked by analyzing the HTS data. Comparing the sequences obtained from different rounds, they discovered the sequence with the highest affinity did not have the highest copy number in the last round [89]. Schultze et al. [90] confirmed this finding when they discovered that the library convergence in SELEX led to high-performance sequences being outcompeted by weaker-performing sequences that amplify more efficiently during PCR. The best binders tend to enrich rapidly in the very early rounds of selection [90]. Spiga et al. [91] performed HTS and SPR to monitor the binding affinity change and aptamer enrichment for tobramycin selection. They also discovered the most enriched and best binding sequences are visible even after two selection rounds [91]. As the cost of HTS continues to decrease [92], more researchers use it for characterizing multiple selection round libraries to ensure the quality of selected candidates [84,93]. Using the HTS dynamic monitoring method, researchers successfully identified high binding aptamers both for proteins [94] and small molecule targets [91].

\subsection{Benchmark Toolkit for HTS SELEX Analysis}

Besides the cost, one of the major hindrances in early years to generalize HTS methods for aptamer identification was the difficulty of processing large amounts of sequence data. However, multiple open-source/paid bioinformatics tools have been developed specifically for aptamer scientists. 
The initial step in processing HTS data from a sequenced SELEX pool is to remove the adapter, barcode, or constant region from the sequences. After this pre-processing, a tool to count the sequence frequency is required. Previously, aptamer research groups used genomic informatics software packages such as Tallymer [95,96] or RazerS [90,97], or designed in-house programs to fulfill the counting requirement [89998].

Galaxy Project is a platform that provides fundamental bioinformatic tools for bench scientists

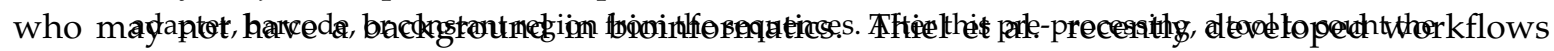

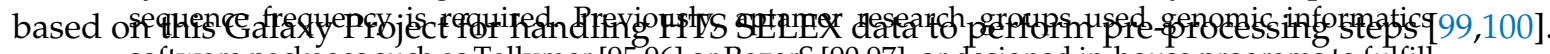

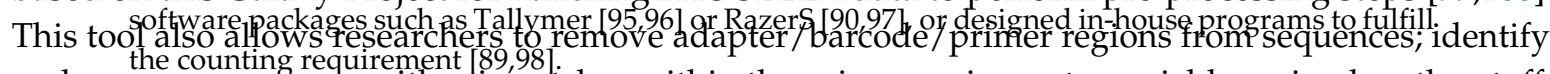
and remove Gequepces w with mismatches within the primer region; set a variable region.length cutoff;

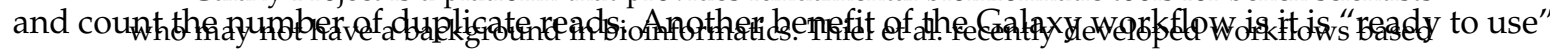

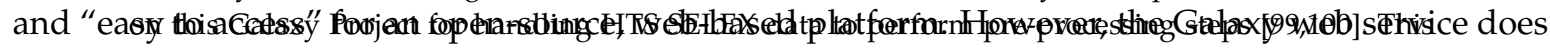
not curteplt genomicprojectequences with mismatches within the primer region; set a variable region length cutoff; and

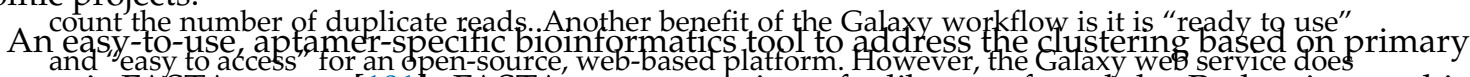

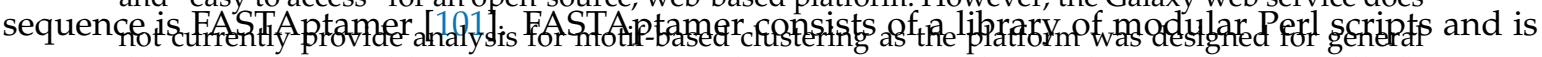

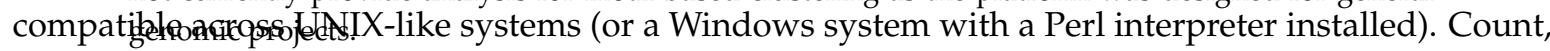

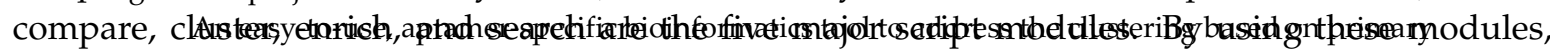

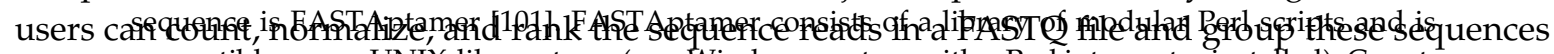

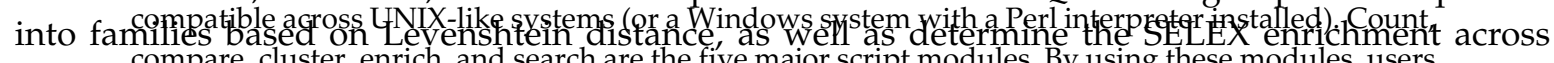

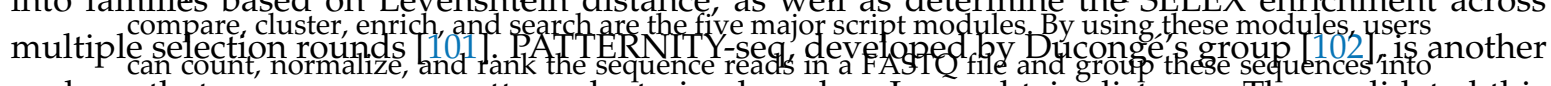

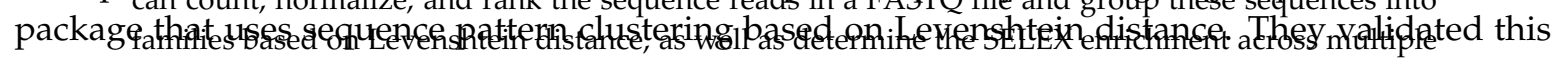

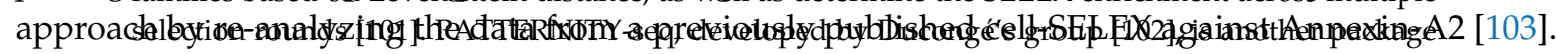

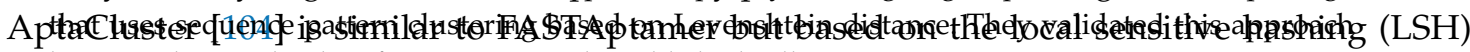

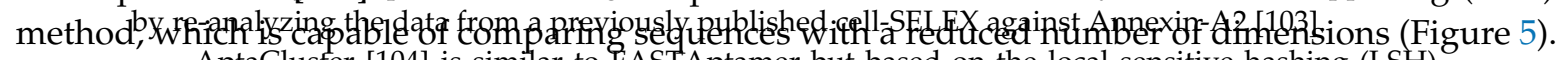
AptaClyster [104l is similar to FASTAptamer but based on the local sensitive hashing (LSH)
Iterative roung

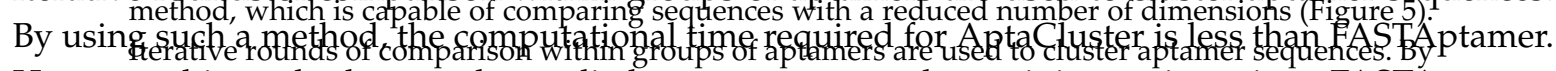

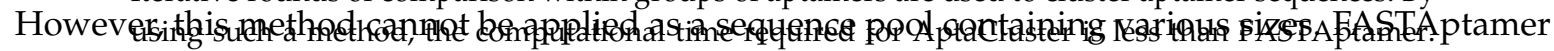

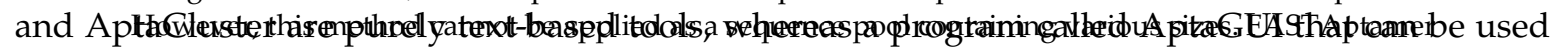

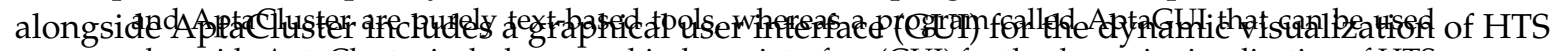

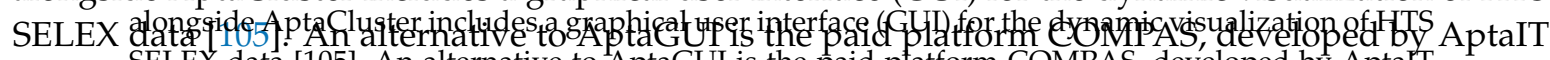
$\mathrm{GmbH}$. ThLEX data [105]. An atternative to AptaGUI is the paid patform COMPAS, developed by Aptart

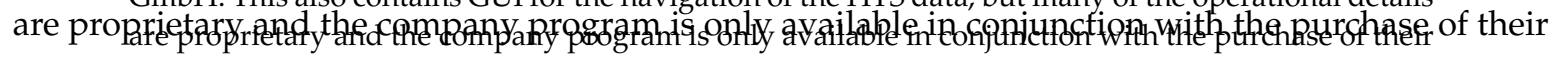
selectionednervice.
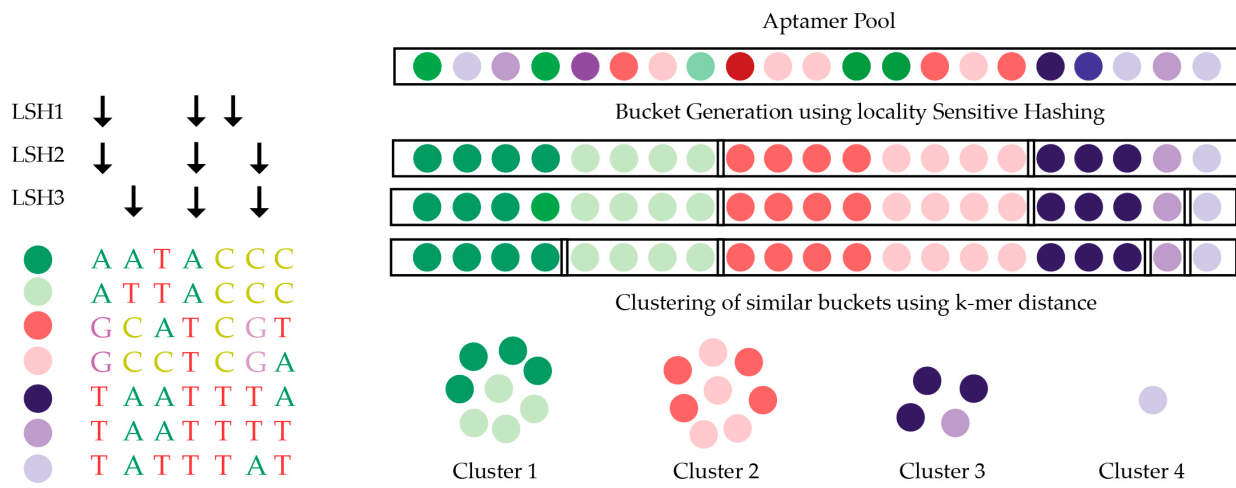

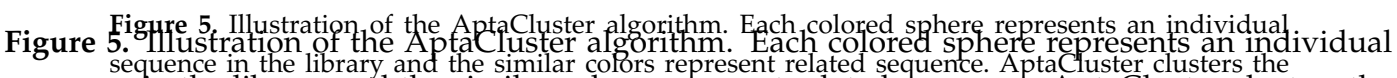

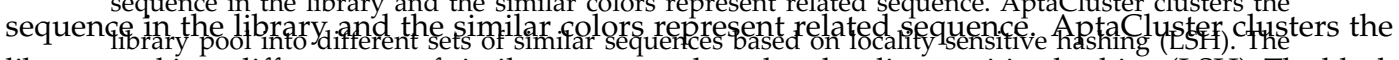

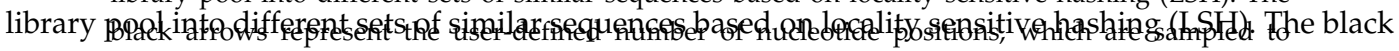

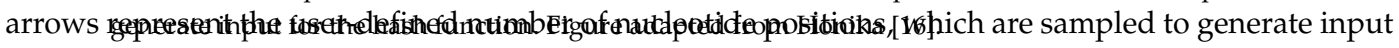
for the hash function. Figure adapted from Honika [16]. 


\subsection{Structure Motif Clustering-Based Tools}

Most of the previously mentioned bioinformatics tools do not include functions for prediction and clustering of HTS data for structure-based methods. Furthermore, they do not allow elucidation of complex motifs and important pre-processing steps for initial analysis of the data generated during SELEX.

Structure prediction programs such as Mfold [106] have been used to analyze low-throughput sequence data. Mfold predicts the secondary structure of single-stranded nucleic acids by energy minimization. Even though "bulk" servers of Mfold can analyze hundreds of sequences at once, it is, however, difficult to handle structure prediction on the HTS scale.

A recently developed platform for structure motif clustering is AptaTrace [107]. Based on the secondary structure prediction from SFOLD [108,109], AptaTrace applies this information into all of the sequences input to the program. This allows for the prediction of a specific structure for each k-mer in each selection round and ranking by predicted significant structural enrichment. APTANI is a similar program, able to cluster sequence motifs based on secondary structure prediction. It uses RNAsubopt from the Vienna RNA package [110] and predicts using sub-structures, apical loops, bulge loops, and intra-strand loops. This method was validated using a SELEX against IL4Ra. Using APTANI, an aptamer was identified in one round, which previously required five rounds.

The speed of HTS technology adoption has motivated the development of particular tools to assist HTS-based SELEX and identify better aptamer candidates (Table 2). Even though many approaches still lack multiple validations, using HTS to replace conventional sequencing methods for aptamer development is the trend. Recent progress in this field shows the potential for developing an all-in-one bioinformatics tools for aptamer researchers.

\section{In Silico Aptamer Optimization}

Aptamers have been isolated with both high affinity and high specificity for binding to their selected targets $[5,111]$. SELEX is an efficient method of isolating aptamers; however, following selection an aptamer scientist must always ask "Have I isolated the best possible aptamer sequence?" The library used for SELEX generally has a random region of around 40 bases [4], and typically only a few nanomoles can be used for the initial selection round. This represents a sequence space coverage of one in 80 billion. From this incredibly small sequence space coverage, it is unlikely that one will select the single best aptamer sequence. Sequence adaptation via mutation may account for some sequence space searching; however, as selection for SELEX is relatively low resolution [112], it is difficult to resolve the very best aptamer sequence.

Bioinformatic approaches have been used to improve the affinity of aptamers. As highlighted earlier, due to low selection pressure classical SELEX is unlikely to resolve the very best aptamer sequences. Therefore, each individual aptamer generated using a bioinformatics approach must be individually assayed for binding affinity, which can be labor-intensive and time-consuming. DNA microarrays consist of many features or spots on a glass slide, each feature containing many copies of a unique DNA sequence. This high-throughput technology allows for simultaneous assay of many aptamer sequences via incubation with fluorescent target.

In 2007, Katilius et al. used DNA microarrays to optimize and explore the surrounding sequence space of an aptamer against immunoglobulin E (IgE) [113]. Variations of the aptamer sequence with single, double and a selection of triple point mutations were synthesized onto a DNA microarray and assayed with Alexa Fluor 647 labeled IgE. This mutational analysis highlighted the conserved and unconserved base positions in the aptamer sequence. One aptamer variant showed mild affinity improvement $\left(K_{\mathrm{D}}=4.1 \mathrm{nM}\right)$ when compared to the original aptamer sequence $\left(K_{\mathrm{D}}=4.7 \mathrm{nM}\right)$ [113].

Platt et al. analyzed the sequence activity relationship of a set of G-quadruplex thrombin binding aptamers using DNA microarray technology [114]. The combinatorial landscape was probed via two methods. The first method investigated two internal loops of the G-quadruplex with 2-3 base random regions (GGGGAGTAGG $\left(X_{2-3}\right)$ GGTGTTGG $\left(X_{2-3}\right)$ GGGGCTCCCC, where $X$ denotes the bases varied). 
The second method investigated the hairpin in which the G-quadruplex is nested within a section using pseudo-random variants $\left(\left(X_{8}\right)\right.$ GGTT $\left(X_{2-4}\right)$ GGTTGGGG $\left(X_{6}\right)$, where $X$ denotes the bases varied). Despite this search through sequence space, the tightest binding novel aptamer $\left(K_{\mathrm{D}}=28 \mathrm{nM}\right)$ had a lower affinity than the original ThB aptamer $\left(K_{D}=26 \mathrm{nM}\right)[114]$.

Knight et al. combined a DNA aptamer microarray assay with in silico closed-loop aptameric directed evolution (CLADE) to select for aptamers against the natively fluorescent target allophycocyanin (APC) [115]. Five hundred control aptamer and 5500 test pool aptamers of 30 nucleotides were synthesized onto a DNA microarray for each round. The initial test pool for the first round was randomly generated. The test pool aptamers were assayed on the microarray for APC binding and ranked according to binding score. The top four aptamers were then subjected to point mutations and insertion-deletion events to give rise to a new 5500 test pool for synthesis onto a DNA microarray and use in the next round of selection. Nine rounds of CLADE were performed and the resulting aptamers characterized and phylogenetically analyzed. The CLADE strategy was successful with the tightest binding aptamer had a SPR determined $K_{\mathrm{D}}$ value of around $2 \mathrm{nM}$ [115]. Although high-affinity aptamers were isolated, the cost of nine microarrays would be much greater than the cost of an average SELEX experiment.

Expanding upon this work, Rowe et al. used the CLADE approach and tested the three diversification systems: mutation, recombination, and statistical binding prediction [116]. Over five CLADE selection rounds, aptamers were evolved to bind to glucose-6-phosphate dehydrogenase. The tightest binding aptamer was isolated using the recombination diversification system and had a $K_{\mathrm{D}}$ of $245 \mathrm{nM}$.

In 2012, Nonaka et al. used an in silico system to improve the affinity of the VEap121 aptamer against VEGF [117]. Interestingly, this study did not use DNA microarrays but instead used SPR to assay every individual aptamer. Three rounds of improvement were performed. Each round consisted of adaptation, SPR determination of $K_{\mathrm{D}}$ value, and selection of the five tightest binding aptamers to seed the next round. For the first generation, 10 mutants of VEap121 were generated, each with several mutations, where the guanine bases were conserved to retain the G-quadruplex structure. For the second generation, the five tightest binding aptamers from G1 as determined by SPR were replicated relative to their binding affinity to yield 20 sequences. These were then randomly paired to undergo single-point crossover and two single-base mutations, randomly introduced. For the third generation, the five tightest binding aptamers from both G1 and G2 as determined by SPR were crossed with VEap121 at a random point and two single-point mutations were randomly introduced. This process was repeated three times in order to produce the third generation of 20 sequences. This process produced four aptamers with a tighter binding $\left(K_{\mathrm{D}}=0.3,1.5,1.7\right.$ and $\left.2.4 \mathrm{nM}\right)$ than the original VEap121 aptamer $\left(K_{\mathrm{D}}=4.7 \mathrm{nM}\right)[117]$.

In 2016 Kinghorn et al. reported a novel strategy of aptamer affinity maturation by library resampling from SELEX sequence data [118]. This approach relies on the principle that classical SELEX is unlikely to select the best possible aptamer, but is highly likely to select family members of the best possible aptamer. The sequence of the best possible aptamer is hidden within the sequences of its family members. To make use of this aptamer family information, the authors coded the bioinformatic software "Resample", which uses information from a SELEX experiment in terms of an aptamer family motif and any available folding information. This information is used to generate a novel library that consists of every possible aptamer permutation within the aptamer family. This library is focused on a particular area of sequence space, representing it thoroughly while still having a library size small enough to fit onto a DNA microarray for screening (Figure 6). To demonstrate this process, the sequence data from a previous selection against the malarial antigen Plasmodium falciparum lactate dehydrogenase (PfLDH) was input into Resample to generate a library of 186,624 novel aptamer sequences within the specified aptamer family. This library was ordered on a DNA microarray that was incubated with $50 \mathrm{nM}$ Alexa Fluor 555 labeled PfLDH (target) and $1 \mu \mathrm{M}$ Alexa Fluor 647 labeled human lactate dehydrogenase B (counter-target) and washed and scanned to measure both binding affinity and binding specificity for all aptamers. The lead candidates were further characterized 
using microscale thermophoresis to show an order of magnitude improvement in binding affinity $K_{\mathrm{D}}$ value. The authors provide Resample as a hosted web service (website available: http: / / resample. azurewebsites.net) and state that affinity maturation using Resample round should just take two

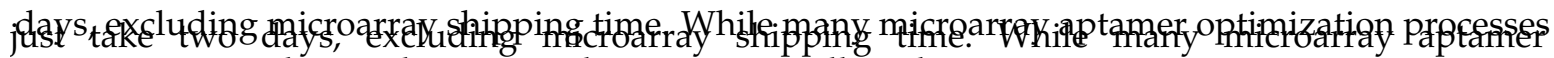

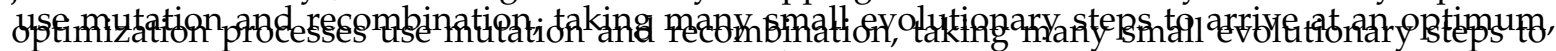

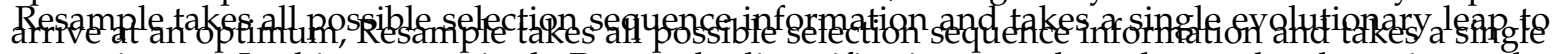

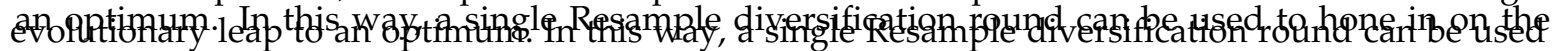

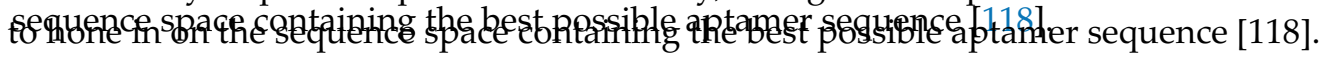

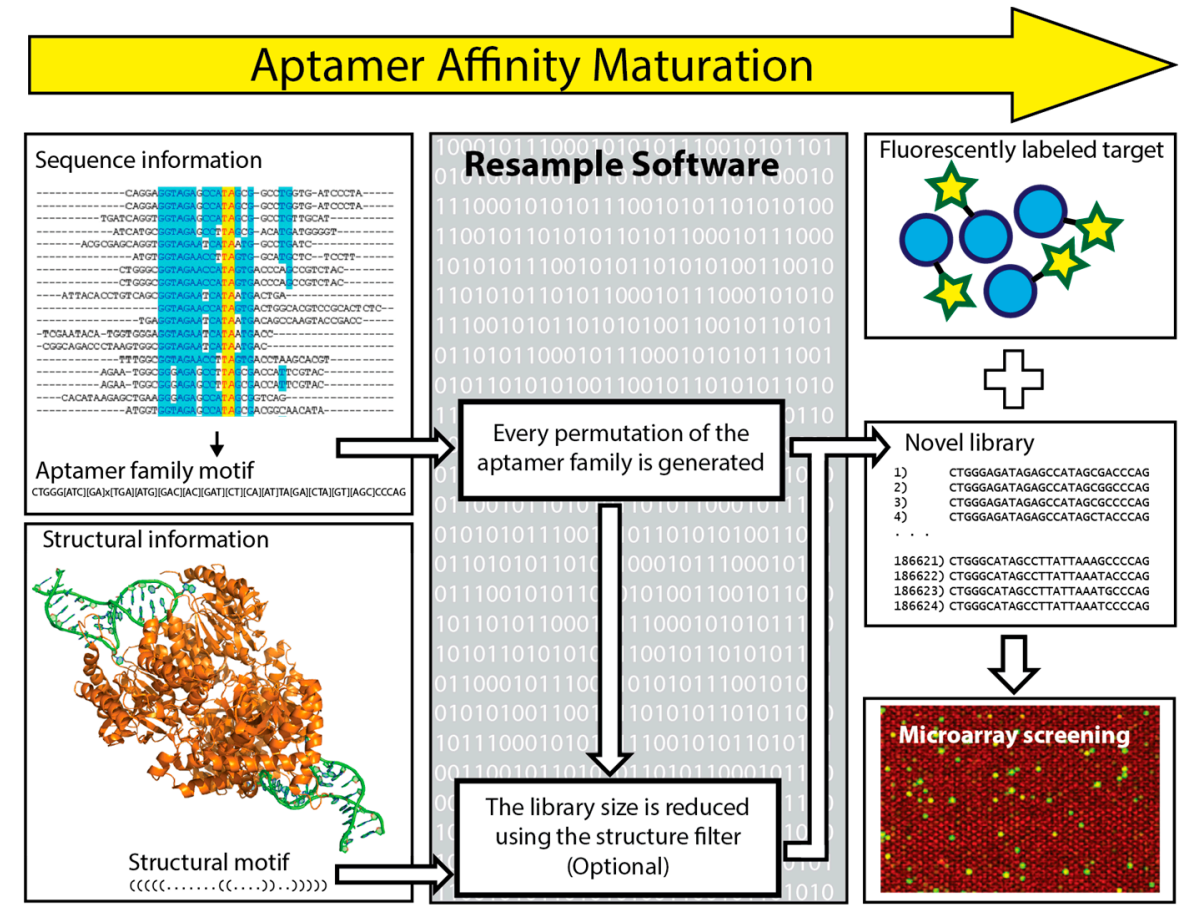

Fisure 6: The aptamer affinity maturation process. The xellow arrow desionates the aptamer affinitity

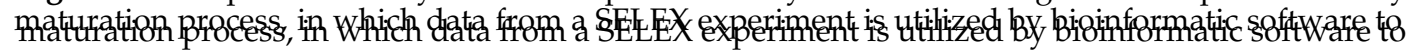

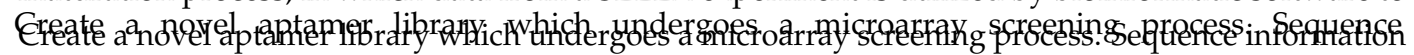
infarmationlable, if

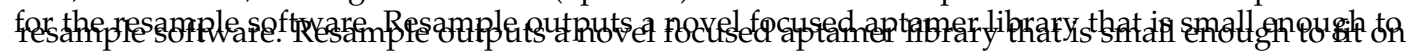

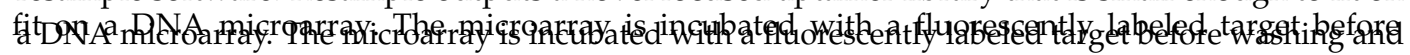

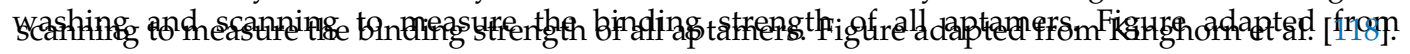
Kinghorn et al. [118].

\section{Conclusions and Future Perspectives
7. Conclusions and Future Perspectives}

Simulation of aptamer selection has given insight into the SELEX process, including the optimum

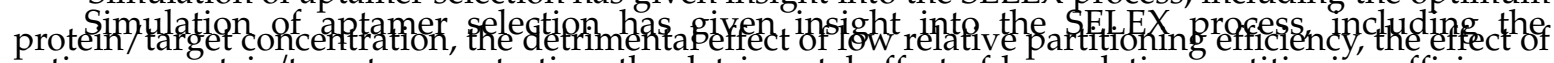

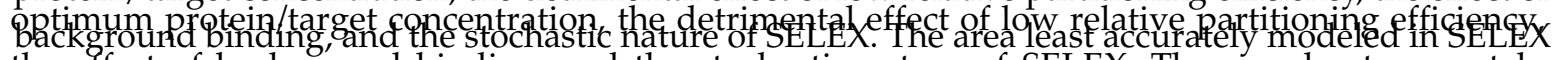
the effect of backoround bindino and the stochastic nature of SELEX The area least accurately modeled in SELEX simulations is aptamer hinding ERexelopment of more accurate aptamer binding models and applying them in simulation may lead to new insights into SELEX In silico approaches have been used to classify molecular interactions between binding hing macromolecules into discrete categories. Each categorized interaction ils assigned a probability and level of importance, which is translated into a score By grouping all the scores, it is ppssible to distinguish apoor binding interaction. from a strong interaction. Most programs have been developed

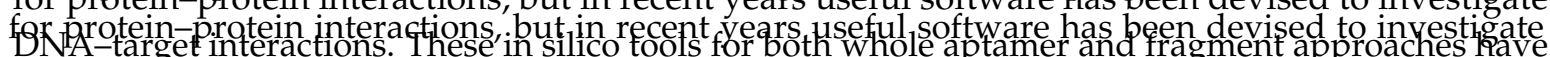
DiNA target interactions. These in silico tools for both whole aptamer and fragment a tiproaches have aided aptamer scientists in improving their selections and identifying novel high-affinity aptamers.

It is commonly known that the huge number of candidates in a random nucleic acid library cannot be covered by a single SELEX experiment. One of the solutions to reduce that number or increase the coverage of selection is to introduce patterns into the library. In silico methods include defining alternating purine and pyrimidine patterns leading to the increase in occurrence of stemloop structures or more complicated structures such as quadruplexes. The design could also be 
It is commonly known that the huge number of candidates in a random nucleic acid library cannot be covered by a single SELEX experiment. One of the solutions to reduce that number or increase the coverage of selection is to introduce patterns into the library. In silico methods include defining alternating purine and pyrimidine patterns leading to the increase in occurrence of stem-loop structures or more complicated structures such as quadruplexes. The design could also be combined with in vitro experiments to access the structural diversity of certain patterned libraries by $C D$ and NMR. Such approaches will effectively help to increase the success rate of identifying active binders in the selection process.

HTS technology shows high potential to replace the cloning and Sanger sequencing methods applied in traditional SELEX. By integrating an HTS step into SELEX, researchers can successfully reduce the selection rounds and the need for post-selection experiments to identify optimal aptamer sequences. These advantages of HTS technology encourage the rapid development of aptamer-based bioinformatic tools. There are several software packages and databases customized for aptamer scientists (Table 2) to analyze the large amount of HTS data based on different strategies. It will be useful to consider how best to compare these tools, using the same batch of data with multiple validations from different research groups.

In silico aptamer optimization has not been widely adopted. This may be due to the observation that most studies either achieve only mild affinity improvements or that the optimization method, while successful, is prohibitively expensive. Nonaka et al. achieved binding improvement of an order of magnitude by using a low-cost method, albeit labor-intensive in SPR measurements [117]. Kinghorn et al. achieved binding improvement of an order of magnitude with a low-cost method that can be performed in two days, excluding microarray shipping time [118]. Many aptamer optimization studies are stand-alones without follow-up or verification by other research groups. For the aptamer community to adopt in silico aptamer optimization, replicate studies need to be performed to strengthen and further validate in silico aptamer optimization methods.

Bioinformatic approaches have been used to improve both aptamers and their selection. In this review we have outlined a broad range of aptamer bioinformatics techniques including simulation of aptamer selection, aptamer selection by molecular dynamics, patterning of libraries, identification of lead aptamers from HTS data, and in silico aptamer optimization. Aptamers are particularly suited to bioinformatic techniques and their development and use can benefit aptamer scientific community.

Acknowledgments: This work was supported in part by the Hong Kong General Research Fund under grants 17127515 and 17163416 to JAT.

Author Contributions: Andrew B. Kinghorn wrote Section 2. Simulation of Aptamer Selection and Section 6. In Silico Aptamer Optimization as well as manuscript editing. Lewis A. Fraser wrote Section 3. Aptamer Selection by Molecular Dynamics as well as manuscript editing. Shaolin Liang wrote Section 5. In Silico Aptamer Identification from High-Throughput Sequencing (HTS) Data as well as manuscript editing. Simon Chi-Chin Shiu wrote Section 4. Patterning of libraries as well as manuscript editing. Julian A. Tanner edited and directed writing of the manuscript.

Conflicts of Interest: The authors declare no conflict of interest.

\section{References}

1. Ellington, A.D.; Szostak, J.W. In vitro selection of RNA molecules that bind specific ligands. Nature 1990, 346, 818-822. [CrossRef] [PubMed]

2. Tuerk, C.; Gold, L. Systematic evolution of ligands by exponential enrichment: RNA ligands to bacteriophage T4 DNA polymerase. Science 1990, 249, 505-510. [CrossRef] [PubMed]

3. Cruz-Toledo, J.; McKeague, M.; Zhang, X.; Giamberardino, A.; McConnell, E.; Francis, T.; DeRosa, M.C.; Dumontier, M. Aptamer base: A collaborative knowledge base to describe aptamers and SELEX experiments. Database 2012. [CrossRef] [PubMed]

4. McKeague, M.; McConnell, E.M.; Cruz-Toledo, J.; Bernard, E.D.; Pach, A.; Mastronardi, E.; Zhang, X.; Beking, M.; Francis, T.; Giamberardino, A. Analysis of in vitro aptamer selection parameters. J. Mol. Evol. 2015, 81, 150-161. [CrossRef] [PubMed] 
5. Cheung, Y.-W.; Kwok, J.; Law, A.W.; Watt, R.M.; Kotaka, M.; Tanner, J.A. Structural basis for discriminatory recognition of Plasmodium lactate dehydrogenase by a DNA aptamer. Proc. Natl. Acad. Sci. USA 2013, 110, 15967-15972. [CrossRef] [PubMed]

6. Irvine, D.; Tuerk, C.; Gold, L. SELEXION: Systematic evolution of ligands by exponential enrichment with integrated optimization by non-linear analysis. J. Mol. Biol. 1991, 222, 739-761. [CrossRef]

7. Vant-Hull, B.; Payano-Baez, A.; Davis, R.H.; Gold, L. The mathematics of SELEX against complex targets. J. Mol. Biol. 1998, 278, 579-597. [CrossRef] [PubMed]

8. Homann, M.; Göringer, H.U. Combinatorial selection of high affinity RNA ligands to live African trypanosomes. Nucleic Acids Res. 1999, 27, 2006-2014. [CrossRef] [PubMed]

9. Coulter, L.R.; Landree, M.A.; Cooper, T.A. Identification of a new class of exonic splicing enhancers by in vivo selection. Mol. Cell. Biol. 1997, 17, 2143-2150. [CrossRef] [PubMed]

10. Chen, C.-K.; Kuo, T.-L.; Chan, P.-C.; Lin, L.-Y. Subtractive SELEX against two heterogeneous target samples: Numerical simulations and analysis. Comput. Biol. Med. 2007, 37, 750-759. [CrossRef] [PubMed]

11. Chen, C.-K. Complex SELEX against target mixture: Stochastic computer model, simulation, and analysis. Comput. Meth. Prog. Biol. 2007, 87, 189-200. [CrossRef] [PubMed]

12. Wang, J.; Rudzinski, J.F.; Gong, Q.; Soh, H.T.; Atzberger, P.J. Influence of target concentration and background binding on in vitro selection of affinity reagents. PLoS ONE 2012, 7, e43940. [CrossRef] [PubMed]

13. Vant-Hull, B.; Gold, L.; Zichi, D.A. Theoretical principles of in vitro selection using combinatorial nucleic acid libraries. Curr. Protoc. Nucleic Acid Chem. 2000. [CrossRef]

14. Zhao, Y.; Granas, D.; Stormo, G.D. Inferring binding energies from selected binding sites. PLoS Comput. Biol. 2009, 5, e1000590. [CrossRef] [PubMed]

15. Spill, F.; Weinstein, Z.B.; Shemirani, A.I.; Ho, N.; Desai, D.; Zaman, M.H. Controlling uncertainty in aptamer selection. Proc. Natl. Acad. Sci. USA 2016. [CrossRef] [PubMed]

16. Hoinka, J.; Berezhnoy, A.; Dao, P.; Sauna, Z.E.; Gilboa, E.; Przytycka, T.M. Large scale analysis of the mutational landscape in HT-SELEX improves aptamer discovery. Nucleic Acids Res. 2015, 43, 5699-5707. [CrossRef] [PubMed]

17. Oh, I.S.; Lee, Y.-G.; McKay, R. Simulating chemical evolution. In Proceedings of the IEEE Congress on Evolutionary Computation, New Orleans, LA, USA, 5-8 July 2011; pp. 2717-2724. [CrossRef]

18. Kauffman, S.; Levin, S. Towards a general theory of adaptive walks on rugged landscapes. J. Theor. Biol. 1987, 128, 11-45. [CrossRef]

19. Wedge, D.C.; Rowe, W.; Kell, D.B.; Knowles, J. In silico modelling of directed evolution: Implications for experimental design and stepwise evolution. J. Theor. Biol. 2009, 257, 131-141. [CrossRef] [PubMed]

20. Deem, M.W.; Lee, H.Y. Sequence space localization in the immune system response to vaccination and disease. Phys. Rev. Lett. 2003, 91, 068101. [CrossRef] [PubMed]

21. Hall, M.; Christensen, K.; di Collobiano, S.A.; Jensen, H.J. Time-dependent extinction rate and species abundance in a tangled-nature model of biological evolution. Phys. Rev. E 2002, 66, 011904. [CrossRef] [PubMed]

22. Kauffman, S.; Macready, W. Technological evolution and adaptive organizations: Ideas from biology may find applications in economics. Complexity 1995, 1, 26-43. [CrossRef]

23. Klussmann, S. Functional oligonucleotides and their applications. In The Aptamer Handbook; John Wiley \& Sons: New York, NY, USA, 2006.

24. Kauffman, S.A. The Origins of Order: Self Organization and Selection in Evolution; Oxford University Press: Oxford, UK, 1992; pp. 61-100.

25. Altenberg, L. Evolving better representations through selective genome growth. In Proceedings of the First IEEE Conference on IEEE World Congress on Computational Intelligence, Evolutionary Computation, Orlando, FL, USA, 27-29 June 1994; pp. 182-187.

26. Kinghorn, A.B.; Tanner, J.A. Selective Phenome Growth Adapted Model: A Novel Landscape to Represent Aptamer Ligand Binding. Complexity 2017, 2017, 1-12. [CrossRef]

27. Wolpert, D.H.; Macready, W.G. No free lunch theorems for optimization. IEEE Trans. Evolut. Comput. 1997, 1, 67-82. [CrossRef]

28. MacKerell, A.D.; Bashford, D.; Bellott, M.; Dunbrack, R.L., Jr.; Evanseck, J.D.; Field, M.J.; Fischer, S.; Gao, J.; Guo, H.; Ha, S. All-atom empirical potential for molecular modeling and dynamics studies of proteins. J. Phys. Chem. B 1998, 102, 3586-3616. [CrossRef] [PubMed] 
29. Durrant, J.D.; McCammon, J.A. Molecular dynamics simulations and drug discovery. BMC Biol. $2011,9,71$.

30. MacKerell, A.D.; Nilsson, L. Molecular dynamics simulations of nucleic acid-protein complexes. Curr. Opin. Struct. Biol. 2008, 18, 194-199. [CrossRef]

31. Gurtovenko, A.A.; Vattulainen, I. Pore formation coupled to ion transport through lipid membranes as induced by transmembrane ionic charge imbalance: Atomistic molecular dynamics study. J. Am. Chem. Soc. 2005, 127, 17570-17571. [CrossRef] [PubMed]

32. Carothers, J.M.; Oestreich, S.C.; Szostak, J.W. Aptamers selected for higher-affinity binding are not more specific for the target ligand. J. Am. Chem. Soc. 2006, 128, 7929-7937. [CrossRef] [PubMed]

33. Gevertz, J.; Gan, H.H.; Schlick, T. In vitro RNA random pools are not structurally diverse: A computational analysis. RNA 2005, 11, 853-863. [CrossRef]

34. Chushak, Y.; Stone, M.O. In silico selection of RNA aptamers. Nucleic Acids Res. 2009, 37, e87. [CrossRef] [PubMed]

35. Hua, W.P.; Linb, H.T.; Tsaia, J.J.; Chenc, W.Y. Investigating interactions between proteins and nucleic acids by computational approaches. In Computational Methods with Applications in Bioinformatics Analysis; World Scientific: Singapore, 2017; p. 98.

36. Arnold, K.; Bordoli, L.; Kopp, J.; Schwede, T. The SWISS-MODEL workspace: A web-based environment for protein structure homology modelling. Bioinformatics 2006, 22, 195-201. [CrossRef] [PubMed]

37. Kelley, L.A.; Mezulis, S.; Yates, C.M.; Wass, M.N.; Sternberg, M.J. The Phyre2 web portal for protein modeling, prediction and analysis. Nat. Protoc. 2015, 10, 845-858. [CrossRef] [PubMed]

38. Van Dijk, M.; Bonvin, A.M. 3D-DART: A DNA structure modelling server. Nucleic Acids Res. 2009, 37, W235-W239. [CrossRef] [PubMed]

39. Popenda, M.; Szachniuk, M.; Antczak, M.; Purzycka, K.J.; Lukasiak, P.; Bartol, N.; Blazewicz, J.; Adamiak, R.W. Automated 3D structure composition for large RNAs. Nucleic Acids Res. 2012, 40, e112. [CrossRef] [PubMed]

40. Setny, P.; Bahadur, R.P.; Zacharias, M. Protein-DNA docking with a coarse-grained force field. BMC Bioinform. 2012, 13, 228. [CrossRef] [PubMed]

41. Das, R.; Baker, D. Automated de novo prediction of native-like RNA tertiary structures. Proc. Natl. Acad. Sci. USA 2007, 104, 14664-14669. [CrossRef] [PubMed]

42. Tsui, V.; Case, D.A. Theory and applications of the generalized Born solvation model in macromolecular simulations. Biopolymers 2000, 56, 275-291. [CrossRef]

43. Zhang, S.; Kumar, K.; Jiang, X.; Wallqvist, A.; Reifman, J. DOVIS: An implementation for high-throughput virtual screening using AutoDock. BMC Bioinform. 2008, 9, 126. [CrossRef] [PubMed]

44. Chen, R.; Li, L.; Weng, Z. ZDOCK: An initial-stage protein-docking algorithm. Proteins 2003, $52,80-87$. [CrossRef] [PubMed]

45. Kumar, J.V.; Chen, W.-Y.; Tsai, J.J.; Hu, W.-P. Molecular simulation methods for selecting thrombin-binding aptamers. In Information Technology Convergence; Springer: Dordrecht, The Netherlands, 2013; Volume 253, pp. 743-749.

46. White, R.R.; Shan, S.; Rusconi, C.P.; Shetty, G.; Dewhirst, M.W.; Kontos, C.D.; Sullenger, B.A. Inhibition of rat corneal angiogenesis by a nuclease-resistant RNA aptamer specific for angiopoietin-2. Proc. Natl. Acad. Sci. USA 2003, 100, 5028-5033. [CrossRef] [PubMed]

47. Sarraf-Yazdi, S.; Mi, J.; Moeller, B.J.; Niu, X.; White, R.R.; Kontos, C.D.; Sullenger, B.A.; Dewhirst, M.W.; Clary, B.M. Inhibition of in vivo tumor angiogenesis and growth via systemic delivery of an angiopoietin 2-specific RNA aptamer. J. Surg. Res. 2008, 146, 16-23. [CrossRef] [PubMed]

48. Hu, W.-P.; Kumar, J.V.; Huang, C.-J.; Chen, W.-Y. Computational selection of RNA aptamer against angiopoietin-2 and experimental evaluation. BioMed Res. Int. 2015, 2015, 658712. [CrossRef] [PubMed]

49. Maisonpierre, P.C.; Suri, C.; Jones, P.F.; Bartunkova, S.; Wiegand, S.J.; Radziejewski, C.; Compton, D.; McClain, J.; Aldrich, T.H.; Papadopoulos, N. Angiopoietin-2, a natural antagonist for Tie2 that disrupts in vivo angiogenesis. Science 1997, 277, 55-60. [CrossRef] [PubMed]

50. Holash, J.; Maisonpierre, P.; Compton, D.; Boland, P.; Alexander, C.; Zagzag, D.; Yancopoulos, G.; Wiegand, S. Vessel cooption, regression, and growth in tumors mediated by angiopoietins and VEGF. Science 1999, 284, 1994-1998. [CrossRef] [PubMed]

51. Lin, H.; Zhang, W.; Jia, S.; Guan, Z.; Yang, C.J.; Zhu, Z. Microfluidic approaches to rapid and efficient aptamer selection. Biomicrofluid 2014, 8, 041501. [CrossRef] [PubMed] 
52. Shangguan, D.; Bing, T.; Zhang, N. Cell-SELEX: Aptamer selection against whole cells, in Aptamers Selected by Cell-SELEX for Theranostics; Springer: Heidelberg, Germany, 2015; pp. 13-33.

53. Mallikaratchy, P. Evolution of Complex Target SELEX to Identify Aptamers against Mammalian Cell-Surface Antigens. Molecules 2017, 22, 215. [CrossRef] [PubMed]

54. Rabal, O.; Pastor, F.; Villanueva, H.; Soldevilla, M.M.; Hervas-Stubbs, S.; Oyarzabal, J. In Silico Aptamer Docking Studies: From a Retrospective Validation to a Prospective Case Study'TIM3 Aptamers Binding. Mol. Ther. Nucleic Acids 2016, 5, e376. [CrossRef] [PubMed]

55. Huang, Y.; Liu, S.; Guo, D.; Li, L.; Xiao, Y. A novel protocol for three-dimensional structure prediction of RNA-protein complexes. Sci. Rep. 2013, 3, 1887. [CrossRef] [PubMed]

56. Berendsen, H.J.; van der Spoel, D.; van Drunen, R. GROMACS: A message-passing parallel molecular dynamics implementation. Comput. Phys. Commun. 1995, 91, 43-56. [CrossRef]

57. Tseng, C.Y.; Ashrafuzzaman, M.; Mane, J.Y.; Kapty, J.; Mercer, J.R.; Tuszynski, J.A. Entropic Fragment-Based Approach to Aptamer Design. Chem. Biol. Drug Des. 2011, 78, 1-13. [CrossRef] [PubMed]

58. Ashrafuzzaman, M.; Tseng, C.-Y.; Kapty, J.; Mercer, J.R.; Tuszynski, J.A. A computationally designed DNA aptamer template with specific binding to phosphatidylserine. Nucleic Acid Ther. 2013, 23, 418-426. [CrossRef]

59. Carothers, J.M.; Oestreich, S.C.; Davis, J.H.; Szostak, J.W. Informational complexity and functional activity of RNA structures. J. Am. Chem. Soc. 2004, 126, 5130-5137. [CrossRef] [PubMed]

60. Davis, J.H.; Szostak, J.W. Isolation of high-affinity GTP aptamers from partially structured RNA libraries. Proc. Natl. Acad. Sci. USA 2002, 99, 11616-11621. [CrossRef] [PubMed]

61. Schultes, E.; Hraber, P.T.; LaBean, T.H. Global similarities in nucleotide base composition among disparate functional classes of single-stranded RNA imply adaptive evolutionary convergence. RNA 1997, 3, 792-806. [PubMed]

62. Knight, R.; De Sterck, H.; Markel, R.; Smit, S.; Oshmyansky, A.; Yarus, M. Abundance of correctly folded RNA motifs in sequence space, calculated on computational grids. Nucleic Acids Res. 2005, 33, 5924-5935. [CrossRef] [PubMed]

63. Ruff, K.M.; Snyder, T.M.; Liu, D.R. Enhanced functional potential of nucleic acid aptamer libraries patterned to increase secondary structure. J. Am. Chem. Soc. 2010, 132, 9453-9464. [CrossRef] [PubMed]

64. Martin, J.A.; Mirau, P.A.; Chushak, Y.; Chavez, J.L.; Naik, R.R.; Hagen, J.A.; Kelley-Loughnane, N. Single-Round Patterned DNA Library Microarray Aptamer Lead Identification. J. Anal. Methods Chem. 2015, 2015, 137489. [CrossRef] [PubMed]

65. Hoogsteen, K. The crystal and molecular structure of a hydrogen-bonded complex between 1-methylthymine and 9-methyladenine. Acta Crystallogr. 1963, 16, 907-916. [CrossRef]

66. Frank-Kamenetskii, M.D.; Mirkin, S.M. Triplex DNA structures. Annu. Rev. Biochem. 1995, 64, 65-95. [CrossRef] [PubMed]

67. De Nicola, B.; Lech, C.J.; Heddi, B.; Regmi, S.; Frasson, I.; Perrone, R.; Richter, S.N.; Phan, A.T. Structure and possible function of a G-quadruplex in the long terminal repeat of the proviral HIV-1 genome. Nucleic Acids Res. 2016, 44, 6442-6451. [CrossRef] [PubMed]

68. Corey, D.R. Telomeres and telomerase: From discovery to clinical trials. Chem. Biol. 2009, 16, 1219-1223. [CrossRef] [PubMed]

69. Bourdoncle, A.; Estévez Torres, A.; Gosse, C.; Lacroix, L.; Vekhoff, P.; Le Saux, T.; Jullien, L.; Mergny, J.L. Quadruplex-Based Molecular Beacons as Tunable DNA Probes. J. Am. Chem. Soc. 2006, 128, 11094-11105. [CrossRef] [PubMed]

70. Zhu, J.; Zhang, L.; Li, T.; Dong, S.; Wang, E. Enzyme-free unlabeled DNA logic circuits based on toehold-mediated strand displacement and split G-quadruplex enhanced fluorescence. Adv. Mater. 2013, 25, 2440-2444. [CrossRef] [PubMed]

71. Webba da Silva, M. Geometric formalism for DNA quadruplex folding. Chemistry 2007, 13, 9738-9745. [CrossRef] [PubMed]

72. Bates, P.J.; Kahlon, J.B.; Thomas, S.D.; Trent, J.O.; Miller, D.M. Antiproliferative activity of G-rich oligonucleotides correlates with protein binding. J. Biol. Chem. 1999, 274, 26369-26377. [CrossRef] [PubMed]

73. Travascio, P.; Li, Y.; Sen, D. DNA-enhanced peroxidase activity of a DNA-aptamer-hemin complex. Chem. Biol. 1998, 5, 505-517. [CrossRef] 
74. Sen, D.; Poon, L.C. RNA and DNA complexes with hemin [Fe(III) heme] are efficient peroxidases and peroxygenases: How do they do it and what does it mean? Crit. Rev. Biochem. Mol. Biol. 2011, 46, 478-492. [CrossRef] [PubMed]

75. Baugh, C.; Grate, D.; Wilson, C. $2.8 \AA$ crystal structure of the malachite green aptamer11Edited by J. A. Doudna. J. Mol. Biol. 2000, 301, 117-128. [CrossRef] [PubMed]

76. Warner, K.D.; Chen, M.C.; Song, W.; Strack, R.L.; Thorn, A.; Jaffrey, S.R.; Ferre-D'Amare, A.R. Structural basis for activity of highly efficient RNA mimics of green fluorescent protein. Nat. Struct. Mol. Biol. 2014, 21, 658-663. [CrossRef] [PubMed]

77. Trachman, R.J., 3rd; Demeshkina, N.A.; Lau, M.W.L.; Panchapakesan, S.S.S.; Jeng, S.C.Y.; Unrau, P.J.; Ferre-D'Amare, A.R. Structural basis for high-affinity fluorophore binding and activation by RNA Mango. Nat. Chem. Biol. 2017, 13, 807-813. [CrossRef] [PubMed]

78. McManus, S.A.; Li, Y. Assessing the amount of quadruplex structures present within G(2)-tract synthetic random-sequence DNA libraries. PLoS ONE 2013, 8, e64131. [CrossRef] [PubMed]

79. Goodwin, S.; McPherson, J.D.; McCombie, W.R. Coming of age: Ten years of next-generation sequencing technologies. Nat. Rev. Genet. 2016, 17, 333-351. [CrossRef] [PubMed]

80. Marian, A.J. Sequencing your genome: What does it mean? Methodist Debakey Cardiovasc. J. 2014, 10, 3-6. [CrossRef] [PubMed]

81. Blind, M.; Blank, M. Aptamer selection technology and recent advances. Mol. Ther. Nucleic Acids 2015, 4, e223. [CrossRef] [PubMed]

82. Berezhnoy, A.; Stewart, C.A.; McNamara, J.O.; Thiel, W.; Giangrande, P.; Trinchieri, G.; Gilboa, E. Isolation and optimization of murine IL-10 receptor blocking oligonucleotide aptamers using high-throughput sequencing. Mol. Ther. 2012, 20, 1242-1250. [CrossRef] [PubMed]

83. Thiel, W.H.; Bair, T.; Peek, A.S.; Liu, X.; Dassie, J.; Stockdale, K.R.; Behlke, M.A.; Miller, F.J., Jr.; Giangrande, P.H. Rapid identification of cell-specific, internalizing RNA aptamers with bioinformatics analyses of a cell-based aptamer selection. PLoS ONE 2012, 7, e43836. [CrossRef] [PubMed]

84. Valenzano, S.; De Girolamo, A.; DeRosa, M.C.; McKeague, M.; Schena, R.; Catucci, L.; Pascale, M. Screening and Identification of DNA Aptamers to Tyramine Using in Vitro Selection and High-Throughput Sequencing. ACS Comb. Sci. 2016, 18, 302-313. [CrossRef] [PubMed]

85. Takahashi, M.; Wu, X.; Ho, M.; Chomchan, P.; Rossi, J.J.; Burnett, J.C.; Zhou, J. High throughput sequencing analysis of RNA libraries reveals the influences of initial library and PCR methods on SELEX efficiency. Sci. Rep. 2016, 6, 33697. [CrossRef] [PubMed]

86. Dupont, D.M.; Larsen, N.; Jensen, J.K.; Andreasen, P.A.; Kjems, J. Characterisation of aptamer-target interactions by branched selection and high-throughput sequencing of SELEX pools. Nucleic Acids Res. 2015, 43, e139. [CrossRef] [PubMed]

87. Zimmermann, B.; Gesell, T.; Chen, D.; Lorenz, C.; Schroeder, R. Monitoring genomic sequences during SELEX using high-throughput sequencing: Neutral SELEX. PLOS ONE 2010, 5, e9169. [CrossRef] [PubMed]

88. Lorenz, C.; Gesell, T.; Zimmermann, B.; Schoeberl, U.; Bilusic, I.; Rajkowitsch, L.; Waldsich, C.; von Haeseler, A.; Schroeder, R. Genomic SELEX for Hfq-binding RNAs identifies genomic aptamers predominantly in antisense transcripts. Nucleic Acids Res. 2010, 38, 3794-3808. [CrossRef] [PubMed]

89. Cho, M.; Xiao, Y.; Nie, J.; Stewart, R.; Csordas, A.T.; Oh, S.S.; Thomson, J.A.; Soh, H.T. Quantitative selection of DNA aptamers through microfluidic selection and high-throughput sequencing. Proc. Natl. Acad. Sci. USA 2010, 107, 15373-15378. [CrossRef] [PubMed]

90. Schütze, T.; Wilhelm, B.; Greiner, N.; Braun, H.; Peter, F.; Mörl, M.; Erdmann, V.A.; Lehrach, H.; Konthur, Z.; Menger, M. Probing the SELEX process with next-generation sequencing. PLoS ONE 2011, 6, e29604.

91. Spiga, F.M.; Maietta, P.; Guiducci, C. More DNA-Aptamers for Small Drugs: A Capture-SELEX Coupled with Surface Plasmon Resonance and High-Throughput Sequencing. ACS Comb. Sci. 2015, 17, 326-333. [CrossRef] [PubMed]

92. Gotrik, M.R.; Feagin, T.A.; Csordas, A.T.; Nakamoto, M.A.; Soh, H.T. Advancements in Aptamer Discovery Technologies. Acc. Chem. Res. 2016, 49, 1903-1910. [CrossRef] [PubMed]

93. Ditzler, M.A.; Lange, M.J.; Bose, D.; Bottoms, C.A.; Virkler, K.F.; Sawyer, A.W.; Whatley, A.S.; Spollen, W.; Givan, S.A.; Burke, D.H. High-throughput sequence analysis reveals structural diversity and improved potency among RNA inhibitors of HIV reverse transcriptase. Nucleic Acids Res. 2013, 41, 1873-1884. [CrossRef] [PubMed] 
94. Scoville, D.J.; Uhm, T.K.; Shallcross, J.A.; Whelan, R.J. Selection of DNA Aptamers for Ovarian Cancer Biomarker CA125 Using One-Pot SELEX and High-Throughput Sequencing. J. Nucleic Acids 2017, 2017, 9879135. [CrossRef] [PubMed]

95. Hoon, S.; Zhou, B.; Janda, K.D.; Brenner, S.; Scolnick, J. Aptamer selection by high-throughput sequencing and informatic analysis. Biotechniques 2011, 51, 413-416. [CrossRef] [PubMed]

96. Kurtz, S.; Narechania, A.; Stein, J.C.; Ware, D. A new method to compute K-mer frequencies and its application to annotate large repetitive plant genomes. BMC Genom. 2008, 9, 517. [CrossRef] [PubMed]

97. Weese, D.; Emde, A.K.; Rausch, T.; Doring, A.; Reinert, K. RazerS-fast read mapping with sensitivity control. Genome Res. 2009, 19, 1646-1654. [CrossRef] [PubMed]

98. Jing, M.; Bowser, M.T. Tracking the emergence of high affinity aptamers for rhVEGF165 during capillary electrophoresis-systematic evolution of ligands by exponential enrichment using high throughput sequencing. Anal. Chem. 2013, 85, 10761-10770. [CrossRef] [PubMed]

99. Thiel, W.H.; Giangrande, P.H. Analyzing HT-SELEX data with the Galaxy Project tools-A web based bioinformatics platform for biomedical research. Methods 2016, 97, 3-10. [CrossRef] [PubMed]

100. Thiel, W.H. Galaxy Workflows for Web-based Bioinformatics Analysis of Aptamer High-throughput Sequencing Data. Mol. Ther. Nucleic Acids 2016, 5, e345. [CrossRef] [PubMed]

101. Alam, K.K.; Chang, J.L.; Burke, D.H. FASTAptamer: A Bioinformatic Toolkit for High-throughput Sequence Analysis of Combinatorial Selections. Mol. Ther. Nucleic Acids. 2015, 4, e230. [CrossRef] [PubMed]

102. Ducongé, F. Improvement of aptamers using PATTERNITY-seq (high-throughput analysis of sequence patterns and paternity relationship between them). In Proceedings of the the Aptamer Bordeaux Conference, Bordeaux, France, 24-25 June 2016.

103. Nguyen Quang, N.; Perret, G.; Duconge, F. Applications of High-Throughput Sequencing for In Vitro Selection and Characterization of Aptamers. Pharmaceuticals 2016, 9, 76. [CrossRef] [PubMed]

104. Hoinka, J.; Berezhnoy, A.; Sauna, Z.E.; Gilboa, E.; Przytycka, T.M. AptaCluster-A Method to Cluster HT-SELEX Aptamer Pools and Lessons from its Application. Res. Comput. Mol. Biol. 2014, 8394, 115-128. [PubMed]

105. Hoinka, J.; Dao, P.; Przytycka, T.M. AptaGUI-A Graphical User Interface for the Efficient Analysis of HT-SELEX Data. Mol. Ther. Nucleic Acids. 2015, 4, e257. [CrossRef] [PubMed]

106. Zuker, M. Mfold web server for nucleic acid folding and hybridization prediction. Nucleic Acids Res. 2003, 31, 3406-3415. [CrossRef] [PubMed]

107. Dao, P.; Hoinka, J.; Takahashi, M.; Zhou, J.; Ho, M.; Wang, Y.; Costa, F.; Rossi, J.J.; Backofen, R.; Burnett, J. AptaTRACE Elucidates RNA Sequence-Structure Motifs from Selection Trends in HT-SELEX Experiments. Cell Syst. 2016, 3, 62-70. [CrossRef] [PubMed]

108. Ding, Y.; Chan, C.Y.; Lawrence, C.E. S fold web server for statistical folding and rational design of nucleic acids. Nucleic Acids Res. 2004, 32, W135-W141. [CrossRef] [PubMed]

109. Chan, C.Y.; Lawrence, C.E.; Ding, Y. Structure clustering features on the $S$ fold Web server. Bioinformatics 2005, 21, 3926-3928. [CrossRef] [PubMed]

110. Hofacker, I.L. RNA secondary structure analysis using the Vienna RNA package. In Current Protoc Bioinformatics; John Wiley \& Sons, Inc.: Hoboken, NJ, USA, 2009.

111. Wang, J.; Gong, Q.; Maheshwari, N.; Eisenstein, M.; Arcila, M.L.; Kosik, K.S.; Soh, H.T. Particle Display: A Quantitative Screening Method for Generating High-Affinity Aptamers. Angew. Chem. Int. Ed. 2014, 53, 4796-4801. [CrossRef] [PubMed]

112. Bowser, M.T. SELEX: Just another separation? Analyst 2005, 130, 128-130. [CrossRef] [PubMed]

113. Katilius, E.; Flores, C.; Woodbury, N.W. Exploring the sequence space of a DNA aptamer using microarrays. Nucleic Acids Res. 2007, 35, 7626-7635. [CrossRef] [PubMed]

114. Platt, M.; Rowe, W.; Knowles, J.; Day, P.J.; Kell, D.B. Analysis of aptamer sequence activity relationships. Integr. Biol. 2009, 1, 116-122. [CrossRef] [PubMed]

115. Knight, C.G.; Platt, M.; Rowe, W.; Wedge, D.C.; Khan, F.; Day, P.J.; McShea, A.; Knowles, J.; Kell, D.B. Array-based evolution of DNA aptamers allows modelling of an explicit sequence-fitness landscape. Nucleic Acids Res. 2009, 37, e6. [CrossRef] [PubMed]

116. Rowe, W.; Platt, M.; Wedge, D.C.; Day, P.J.; Kell, D.B.; Knowles, J. Convergent evolution to an aptamer observed in small populations on DNA microarrays. Phys. Biol. 2010, 7, 036007. [CrossRef] [PubMed] 
117. Nonaka, Y.; Yoshida, W.; Abe, K.; Ferri, S.; Schulze, H.; Bachmann, T.T.; Ikebukuro, K. Affinity improvement of a VEGF aptamer by in silico maturation for a sensitive VEGF-detection system. Anal. Chem. 2012, 85, 1132-1137. [CrossRef] [PubMed]

118. Kinghorn, A.B.; Dirkzwager, R.M.; Liang, S.; Cheung, Y.-W.; Fraser, L.A.; Shiu, S.C.-C.; Tang, M.S.; Tanner, J.A. Aptamer Affinity Maturation by Resampling and Microarray Selection. Anal. Chem. 2016, 88, 6981-6985. [CrossRef] [PubMed] 


\title{
Oligonucleotide Functionalised Microbeads: Indispensable Tools for High-Throughput Aptamer Selection
}

\author{
Lewis A. Fraser ${ }^{\dagger}$, Andrew B. Kinghorn ${ }^{\dagger}$, Marco S. L. Tang, Yee-Wai Cheung, Bryce Lim, \\ Shaolin Liang, Roderick M. Dirkzwager and Julian A. Tanner* \\ Received: 22 September 2015 ; Accepted: 12 November 2015 ; Published: 1 December 2015 \\ Academic Editors: Alain O. A. Miller and Jean Jacques Vanden Eynde \\ School of Biomedical Sciences, Li Ka Shing Faculty of Medicine, The University of Hong Kong, Pokfulam, \\ Hong Kong, China; lewis-fraser@hku.hk (L.A.F.); andrew.b.kinghorn@gmail.com (A.B.K.); \\ szelokt@hku.hk (M.S.L.T.); cheungw@hku.hk (Y.-W.C.); bl404@cam.ac.uk (B.L.); shaolin2@hku.hk (S.L.); \\ rdirkz@hku.hk (R.M.D.) \\ * Correspondence: jatanner@hku.hk; Tel.: +852-3917-9472; Fax: +852-2855-1254 \\ + These authors contributed equally to this work.
}

\begin{abstract}
The functionalisation of microbeads with oligonucleotides has become an indispensable technique for high-throughput aptamer selection in SELEX protocols. In addition to simplifying the separation of binding and non-binding aptamer candidates, microbeads have facilitated the integration of other technologies such as emulsion PCR (ePCR) and Fluorescence Activated Cell Sorting (FACS) to high-throughput selection techniques. Within these systems, monoclonal aptamer microbeads can be individually generated and assayed to assess aptamer candidate fitness thereby helping eliminate stochastic effects which are common to classical SELEX techniques. Such techniques have given rise to aptamers with 1000 times greater binding affinities when compared to traditional SELEX. Another emerging technique is Fluorescence Activated Droplet Sorting (FADS) whereby selection does not rely on binding capture allowing evolution of a greater diversity of aptamer properties such as fluorescence or enzymatic activity. Within this review we explore examples and applications of oligonucleotide functionalised microbeads in aptamer selection and reflect upon new opportunities arising for aptamer science.
\end{abstract}

Keywords: aptamer; SELEX; microbead; monoclonal bead; ePCR; FACS

\section{Introduction}

Aptamers are synthetic oligonucleotides that fold into unique 3-D structures that specifically bind to their targets with high affinity. Since aptamers were first described by two independent research teams in 1990 [1,2], they have been rapidly adapted for various applications in research and biotechnology. Aptamers can be selected against a wide variety of targets including metal ions [3,4], small molecules [5,6], proteins [7,8], and even whole cells [9,10]. Aptamers offer multiple advantages when compared to the analogous protein based affinity reagents, antibodies. These advantages include greater stability, specificity, ease of chemical modification, lower production cost and less batch to batch variability [11]. Aptamers are typically selected from a random library through a process called systematic evolution of ligands by exponential enrichment (SELEX). Figure 1 (Orange) shows the basic method for obtaining an aptamer by SELEX. Generally, SELEX comprises the iterative steps of aptamer library binding, elution of bound species and pool amplification until aptamer candidates with desired criteria are selected. A random nucleic acid library is incubated with a target. Sequences which bind to the target are then partitioned and any non-binding or low 
Molecules 2015, 20, page-page affinity sequences are discarded. Binding sequences are eluted and amplified by the polymerase

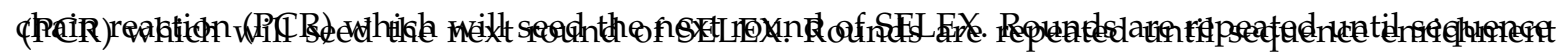

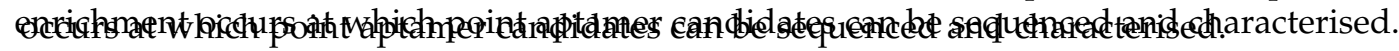

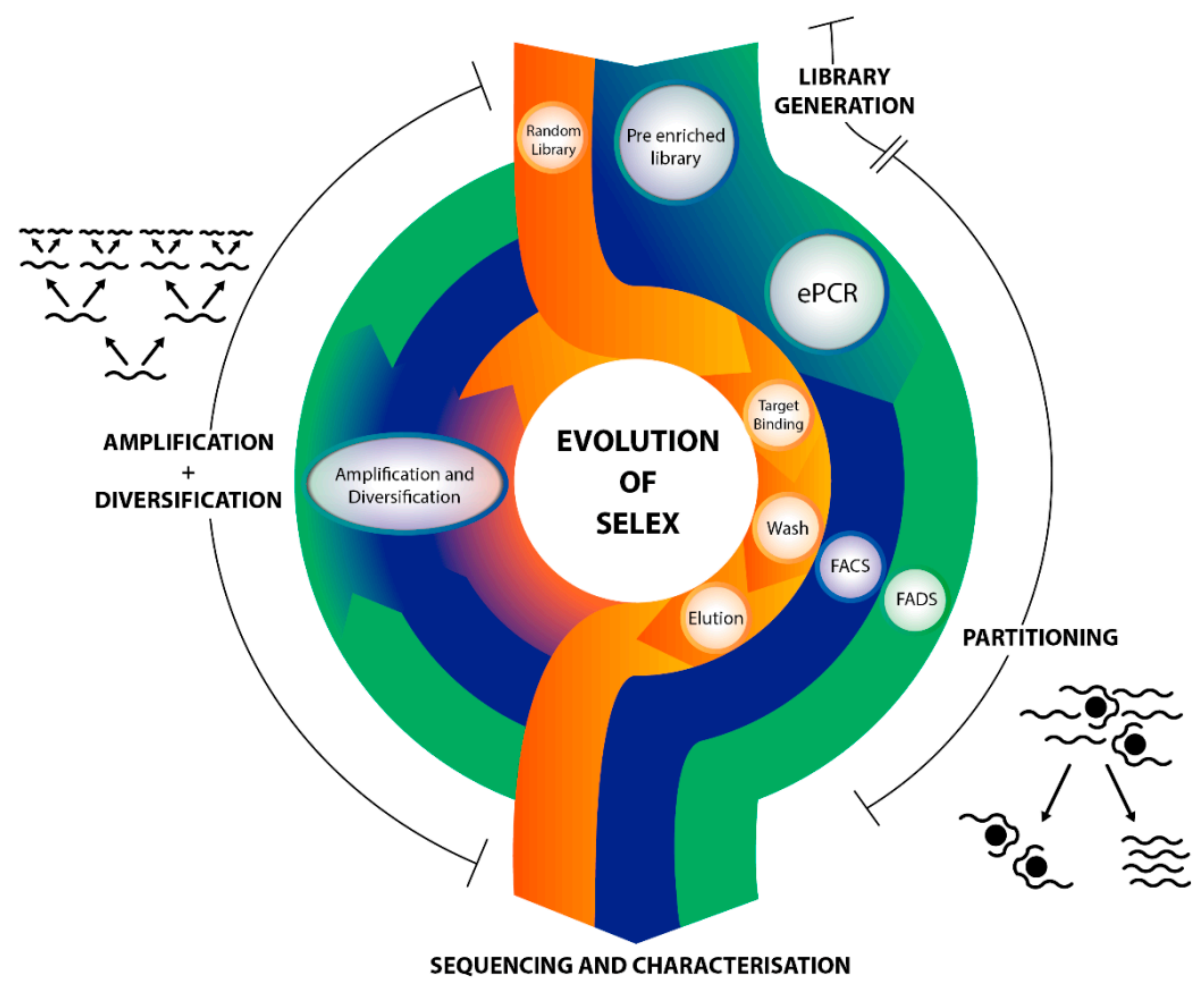

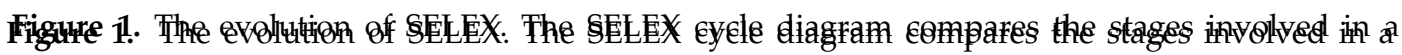

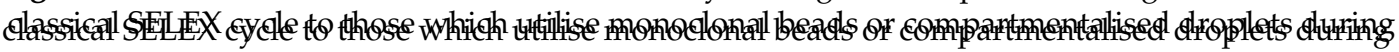

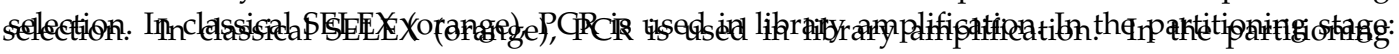

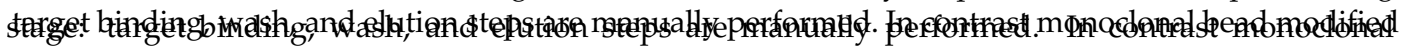

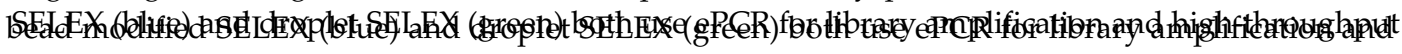

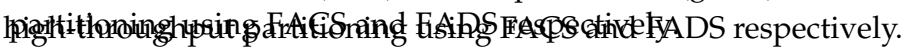

Elassical SELEX is a well-estahlished approach, however it is labour intensive and time-consuming. As is common of any evolutionary process classical SÉLEX is sensitive to stochastic effects that influence the felection process. These effects can in tude loss of tight bindipg members retentipn of weak binders or random fluctuation sin amplification. In the first found of selection there may he as low as one copy of any given aptamer candidate, resulting in the loss of ideal binders and. unw anted selection of spurious sequences. Even though the effects werefon wored in early SEL EX numerical and theoretical simulation experiments [12,13] there is 3 rowing evidence to support that stochastic effects can substantially affect selection results, especially when dealing with complex taropets [14, 15]. As a result several techniques have been developed to improve selection. These involve the isolation and amplification of individual, sequences such that sufficient binding events occur per sequence candidate to allow for detectable assays to be employed [16-18]. By assaying each individual member ind the library the stochasticeffect is greatly reduced

Microbeads are small amenable particles that are widely used in both industry and research. They comprise of solid, gel-like or vesicular particles with sizes ranging from nanometre to millimetre diameters [19]. Magnetic microbeads havebeen used extensively in classical SELEX experiments for drameters [19]. Magnetic microbeads have been used extensively in classical setex experiments for immobilising selection targets, including our own experience [3, 20,21]. This substantially simplifies the partitioning of target-bound and -unbound species dyring selection. Since the first application of magnetic micr.obeads for aptamer selection in 1996 [22] more sophisticated technologies which harness the potential of microbeads in optimising and streamlining SELEX experiments have been developed. Therefore this review will focus on the impact such microbead technologies have had on aptamer research. 
harness the potential of microbeads in optimising and streamlining SELEX experiments have been developed. Therefore this review will focus on the impact such microbead technologies have had on aptamer research.

Microbeads have the ability to be decorated with oligonucleotides of a single species. Despite inherent drawbacks associated with surface immobilisation of aptamers [23], monoclonal beads have greatly enhanced partitioning (Figure 1) during the aptamer selection process. In classical SELEX the partitioning process is made up of target binding, washing and elution (Figure 1, Orange), all of which are stochastic in nature and lead to loss of fit sequences. The notion of characterising every aptamer in an early round SELEX is daunting yet would eliminate the single molecule stochastic effect problems limiting the partitioning process. A promising method to limit these factors is monoclonal microbead technology. Flow cytometry or FACS technology is currently capable of sorting cells and beads at rates of up to around $10^{8}$ per hour [24]. By combining FACS technology with pre-enriched monoclonal bead libraries, each individual aptamer candidate can be assayed and partitioned automatically in a high-throughput manner (Figure 1, Blue) [18], thereby minimising the stochastic effects encountered in classical selection techniques. This transition from binding capture, vulnerable to stochastic effects, to FACS selection is a major step forward in terms of selection efficiency and efficacy. This improvement is best illustrated by the 1000 fold increase in binding strength achieved when aptamers for a certain target were selected using FACS instead of classical techniques [18]. This review therefore considers the various theoretical and experimental considerations involved in these new microbead-based selection technologies.

Aptamer research has primarily focused on discovery of oligonucleotide sequences with binding properties, however molecular binding represents a fraction of their functional potential. Examples from biology-including, most famously, the ribosome-exemplify the vast catalytic potential of oligonucleotides. Ribozymes, DNAzymes and XNAzymes have been developed in vitro to perform simple enzymatic functions whereby clever strategies need to be used to link product to reactant during the selection [25]. Microbeads show potential in developing higher complexity enzymatic interactions by combining monoclonal encapsulation and FADS technology (Figure 1, Green).

\section{Functionalised Bead Generation}

Methods for the generation of oligonucleotide-functionalised monoclonal microbeads have been pivotal in advancing aptamer selection techniques. These monoclonal beads can be produced using several different techniques, the most promising of which uses ePCR. To date, several examples of aptamer selections incorporating monoclonal beads generated by ePCR have been reported (Figure 1, Blue) $[18,26,27]$. This section therefore seeks to fully explore the different techniques involved in monoclonal bead generation for the application of aptamer selection and primarily focuses on the promising area of ePCR.

\subsection{One-Bead-One-Oligonucleotide Library}

Combinatorial chemistry has enabled the simple construction of monoclonal bead-based oligonucleotide libraries by the "split synthesis" method [28]. The "one-bead-one-compound" concept was originally developed for synthetic peptide library generation [29], and was subsequently expanded to the generation of oligonucleotide [30] and non-peptide oligomeric compounds [31]. These combinatorial libraries contain single beads each displaying $10^{13}$ copies of a single compound, thereby facilitating the assaying of single beads in downstream applications. The first one-bead-one-oligonucleotide library for SELEX was used to select synthetic phosphodiester-modified oligonucleotides against transcription factor NF-kB p50/p50 protein in combination with FACS screening [30,32]. However, application of combinatorial oligonucleotide library generation directly onto beads remains limited in SELEX since only a small library can be represented. Fully representing a 20-nucleotide sequence would require $10^{12}$ individual beads to 


\subsection{Emulsion Polymerase Chain Reaction (ePCR)}

Unlike the direct synthesis of oligonucleotide libraries to bead, ePCR enables multiple round Wrondemal 2bead9\$ELZEX. In vitro compartmentalisation (IVC) using water in oil (W/O) emulsion technology was developed in 1998 [33] for the in vitro evolution of enzymes. In this technique, picolitre volume aqueous droplets in an oil medium are used as miniature reaction vessels. Due to

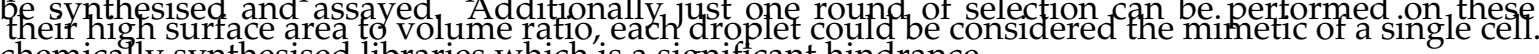
chemically synthesised libraries which is a signiflcant hindrance. 2.2. . oligonucleotide decorated monoclonal microbeads within droplets without any cross-reactivity.

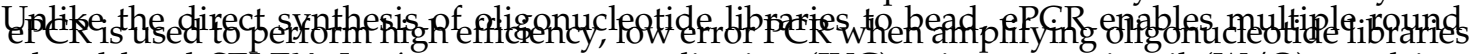
m39n.

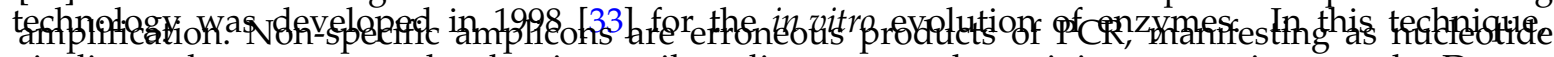

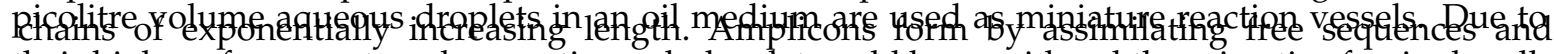

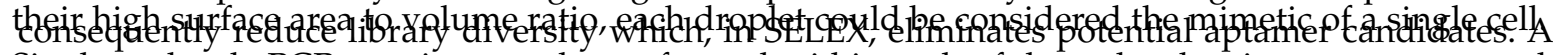

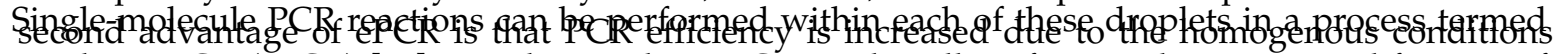

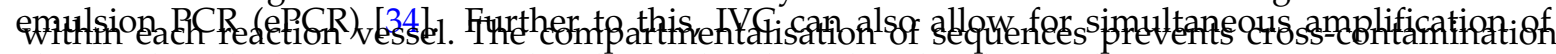

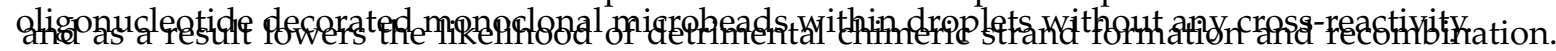

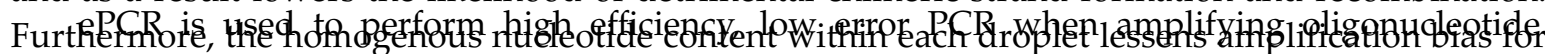

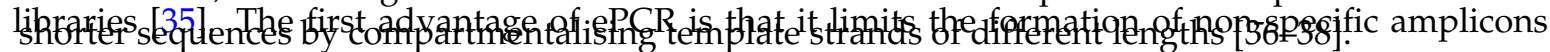
during amplification. Non-specific amplicons are erroneous products of PCR, manifesting as

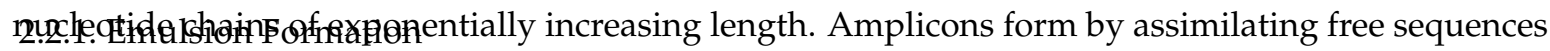
and consequently reduce library diversity which, in SELEX, eliminates potential aptamer candidates.

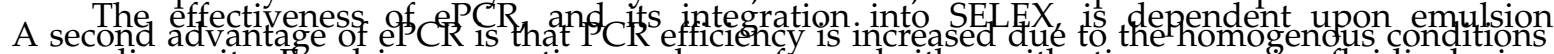
monodispersity. Emulsion, geperation can be performed either with stirrers or microfluidic devices (Figure 2). Both methods reliken the Poisson distribution to predict and maximise the proportion of droplets that contain exactly one sequence as a function of sequence concentration [39]

Furthermore, the homogenous nucleotide content within each droplet lessens amplification bias for Certain methods of emulsion formation use stirrers to ocenerate surfactant stabilised water
shorter sequences by compartmentalising template strands of different lengths $36-38$. droplets in an oil phase (Figure 2a) [34]. The primary drawback to such methods is the large variability

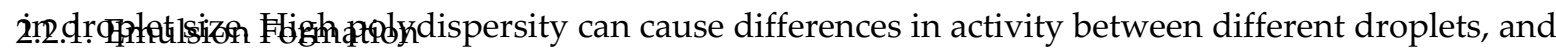
increases the uncertainty when predicting the proportion of droplets that contain exactly one sequence effectiveness of ePCR and its integration into SELEX is dependent uppon emulsion

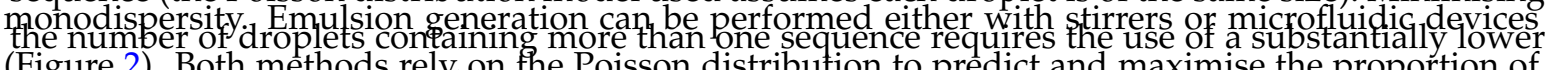
(Figure 2). Both methods rely on he Poisson distribution to predict and maximise the proportion of
seguence concentration which in turn results in a larger number of empty droplets 140 . droplets that contain exactly one sequence as a function of sequence concentration [39].

(ต)

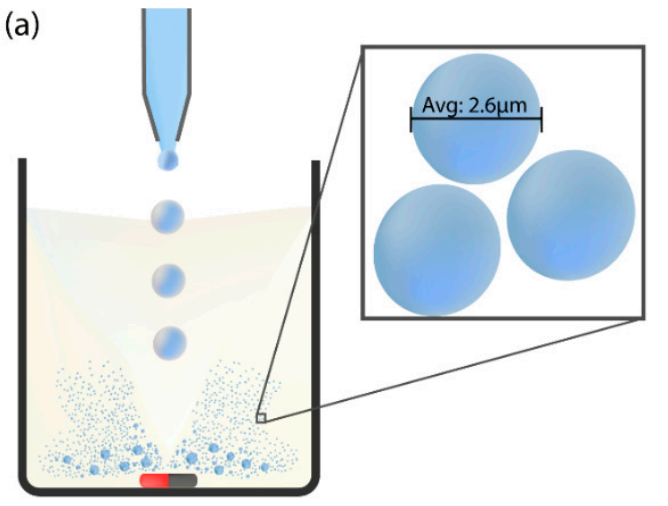

(b)

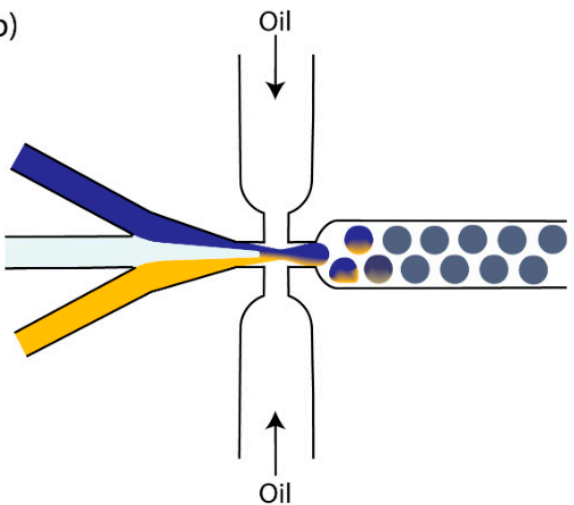

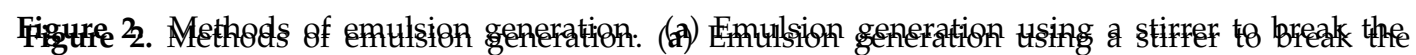

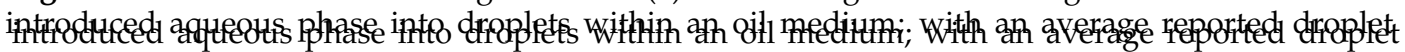

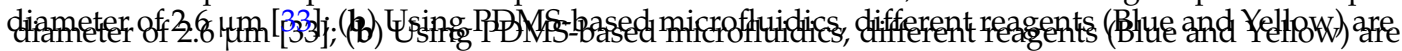

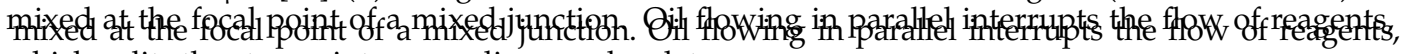
which splitts the stream intemonodisperse dropletss.

Certain methods of emulsion formation use stirrers to generate surfactant stabilised water droplets in an oil phase (Figure 2a) [34]. The primary drawback to such methods is the large variability in droplet size. High polydispersity can cause differences in activity between different droplets, and increases the uncertainty when predicting the proportion of droplets that contain 
exactly one sequence (the Poisson distribution model used assumes each droplet is of the same size). Minimising the number of droplets containing more than one sequence requires the use of

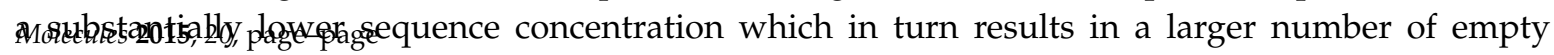
droplets [40].

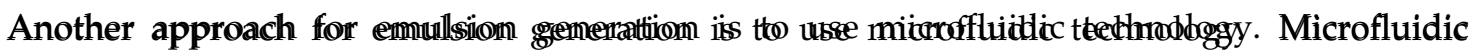
platforms for emulsion generation typically only require a cross-jumction of chammells [41]. At certain junctions droplet reagents in laminar flow can be combined into droplets at regular intervals by the constant flow of an immiscible separation liquid such as silicon oil (Figure 2b). Microfluidic devices are typically made of polydimethylsiloxane (PDMS). The soft lithography involved in forming microfluidic emulsion forming devices involve the use of a clean rooms, moulds, and specialised

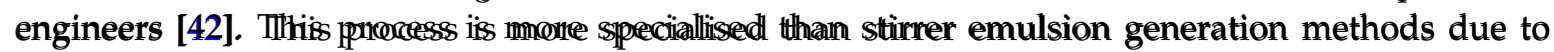
additional equipment and expertise required for the microfluidic droplet setup, including pumping equipment, tubing, and microscopes [41], which himders itts mone rviddespread usee.

\section{2:2:2: Uses of ePCR and Microbeads for Molecular Recognition Analysis and Aptamer Selection}

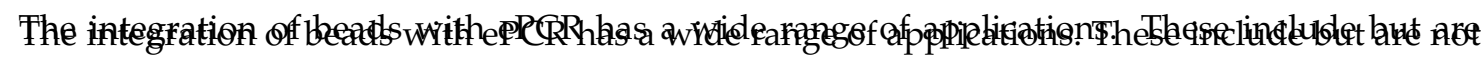

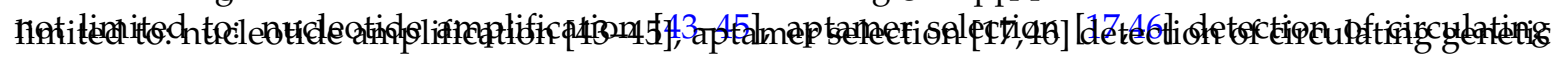

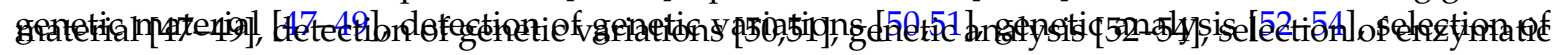

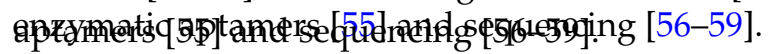

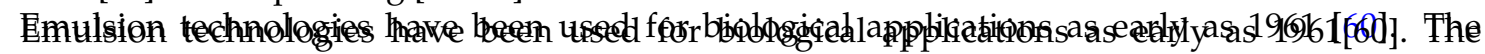
first uses of droplet compartmentalised biological reactions were in evolutionary experiments, these investigated in zitro linkage for the directed evolution of enzymes [33,61,62]: These experiments were fundamental to the subsequent development of ePCR (Tigure 3) [34]: The first use of ePCR in aptamer selection was generation of monoclonal DNA droplets for high quality oligonucleotide libraries [36].

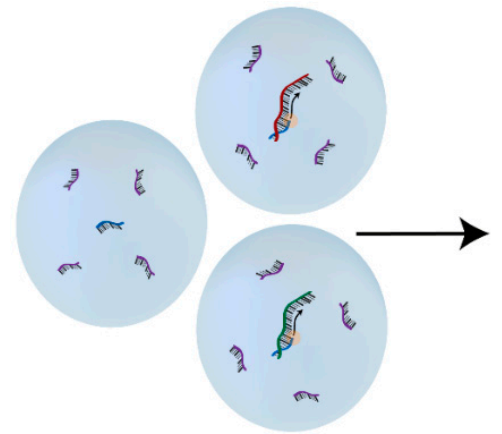

Forward Primer Hybridisation and Strand Amplification

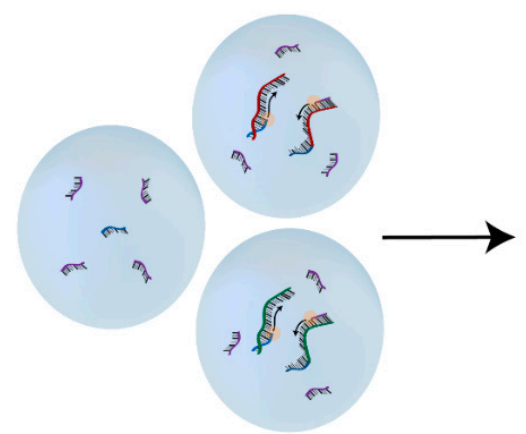

Reverse Primer Hybridisation and Strand Amplification

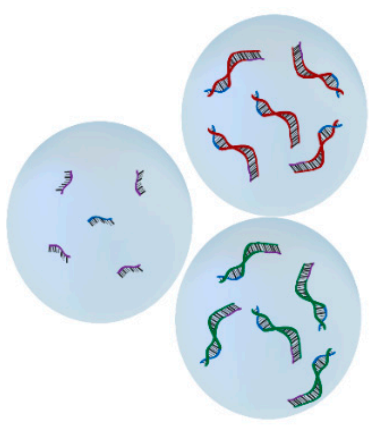

Droplets Containing Monoclonal DNA

Eigure 3. Emulsion Polymerase Chain Reaction. Proplets are formed containing PCR reagents, including forward primers (Blue strands) and reverse primers (Purple Strands) and femplate strands (Red and Green strands): Goncentrations are adjusted to ensure that each reacting droplet contains

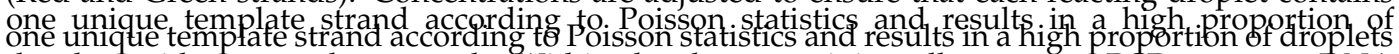
droplets without template strands. Within droplets containing all Hecessary PQR reagentso DNA
without template strards. Withind amplification occurs. PCR amplification is cycled until the droplets contain an optimal concentration
occirs. strdouble stranded monoglonal DNA.

The use of microbeads within ePCR (Figure 4) has greatly benefited the assaying of molecular

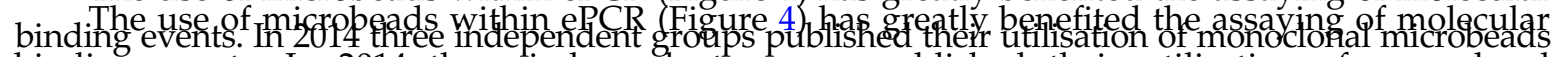

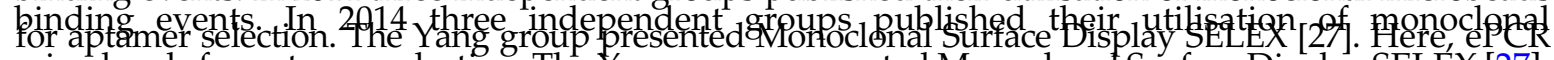
microbeads for aptamer selecton. The Yang grouppresented Monoclonal Surface Display SELEX. S27]. Here ePCR was used to form monoclonal beads that bind to proteins and cell thar targets in the

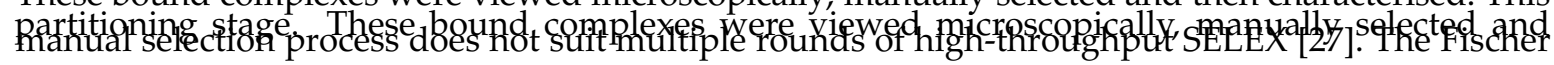
group used Beads Emulsion, Amplification and Magnetics (BEAMing) for both library amplification and strand separation stages to "Just-in-time SELEX" [26]. The Soh group presented their Particle Display technique [18], which successfully integrated monoclonal beads into all stages of SELEX (Figure 1, Blue): library generation, intra-round amplification, and for FACS based partitioning and characterisation-discussed in detail in the section below. 
then characterised. This manual selection process does not suit multiple rounds of high-throughput SELEX [27]. The Fischer group used Beads Emulsion, Amplification and Magnetics (BEAMing) for both library amplification and strand separation stages to "Just-in-time SELEX" [26]. The Soh group presented their Particle Display technique [18], which successfully integrated monoclonal beads into all stages of SELEX (Figure 1, Blue): library generation, intra-round amplification, and for FACS based partitioning and characterisation-discussed in detail in the section below.

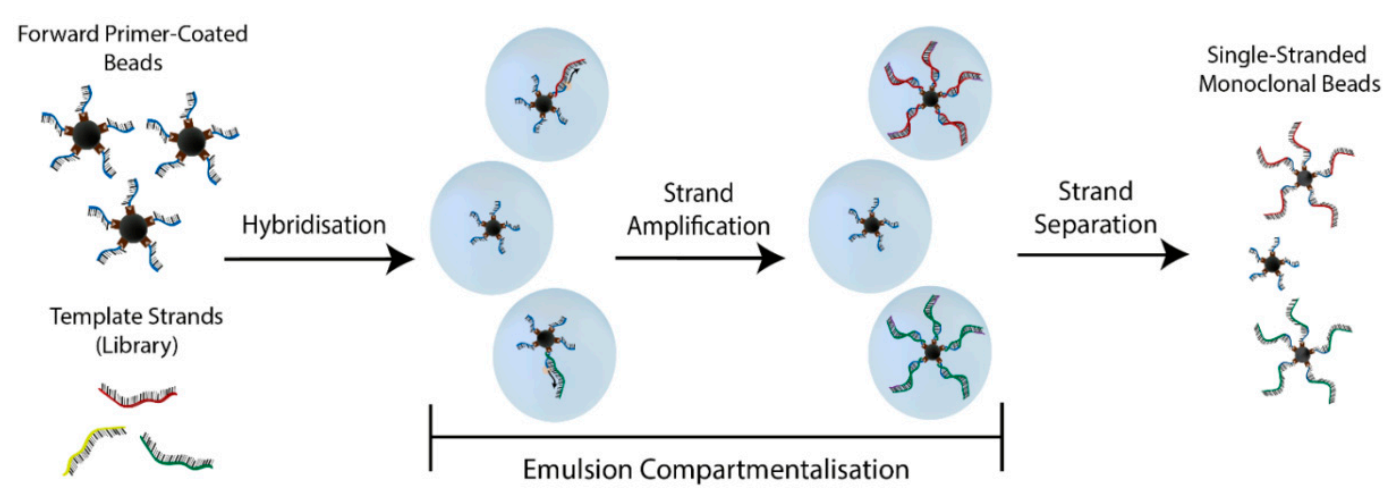

Figure 4. Schematic of bead-based eBER: Step 1: Primer-coated beads and template library are combined in an emulsion, with one bead and one unique template strand per reacting droplet stepe

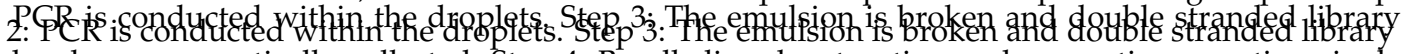
beads are magnetically collected. Step 4: By alkaline denaturation and magnetic separation single stranded monoclonal beads are formed. On average only $110 \%$ of droplets will contain sequences with ass many ass $80 \%$ of nucleotide coated beads being monoclonal [18].

\section{Partitioning and Characterisation}

Partitioning of binding from non-binding aptamers in classical SELEX invglves target binding,
Partitioning of binding from non-binding aptamers in classical SELEX involves target binding, washing to remove weak binders and elution of tight binders (Figure 1). All three of these partitioning steps are stochastic.in nature. This can result.in the loss of beneficial tioht steps are stochastic in nature. This can result in the loss of beneficial tignt-binding aptamer sequences, especially in earlier SELEX rounds. The solution to this problem poses a significant experimental challenge of single molecule interaction detection. However by amplifining an aptamer sequence to an IV fver monoclonal gligonucleotide bead this undetectably small single molequle interaction is sufficiently amplified for the mass interaction on the bead to be detectable hn this way throtgh IV. IV monoclonal surface displav single aptamer inteactions can be detected, assayed, sorted and selected in a lens stochastic manner.
selected in a less stochastic manner.

\subsection{Flutrescence Activated Eell Sorting (FAEGS)}

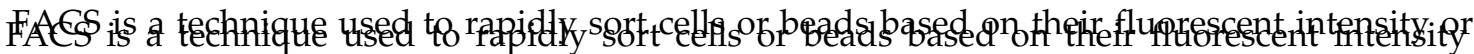

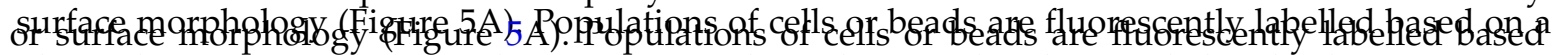

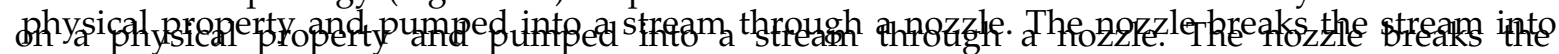

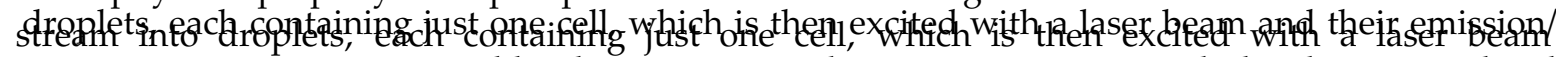

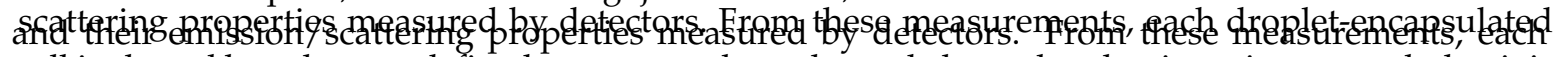

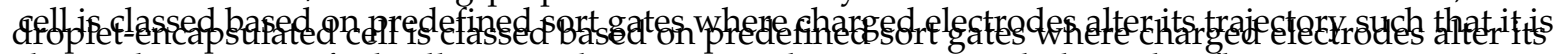

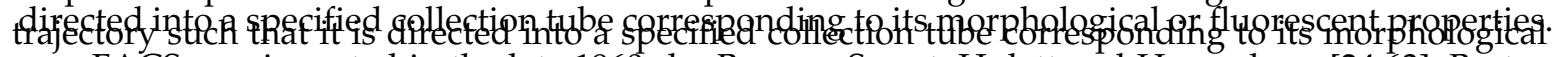
or flubAcSentasiopyentied in the late 1960s by Bonner, Sweet, Hulett and Herzenberg $[24,63]$. Becton

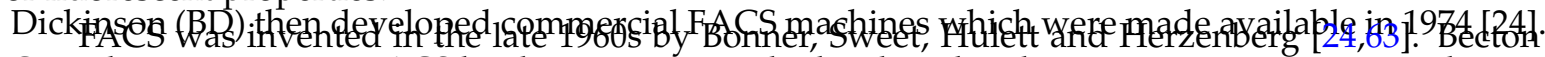
Bickrthenn

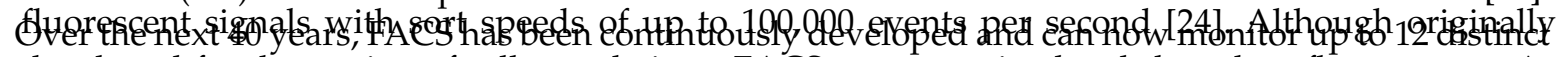

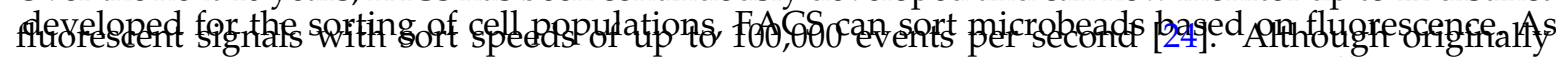

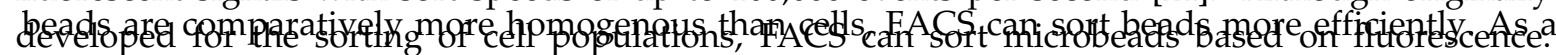
result FACS is an ideal technology for use in selection of large libraries of bead bound aptamers against fluorescent targets. Furthermore since every member of a bead bound aptamer library is individually analysed, the stochastic nature of the 203 inding/capture SELEX $[13,64]$ is eliminated. 
As beads are comparatively more homogenous than cells, FACS can sort beads more efficiently. As a result FACS is an ideal technology for use in selection of large libraries of bead bound aptamers against fluorescent targets. Furthermore since every member of a bead bound aptamer library is indidides 2015 , analysegdgthe stochastic nature of the binding/capture SELEX $[13,64]$ is eliminated.

A

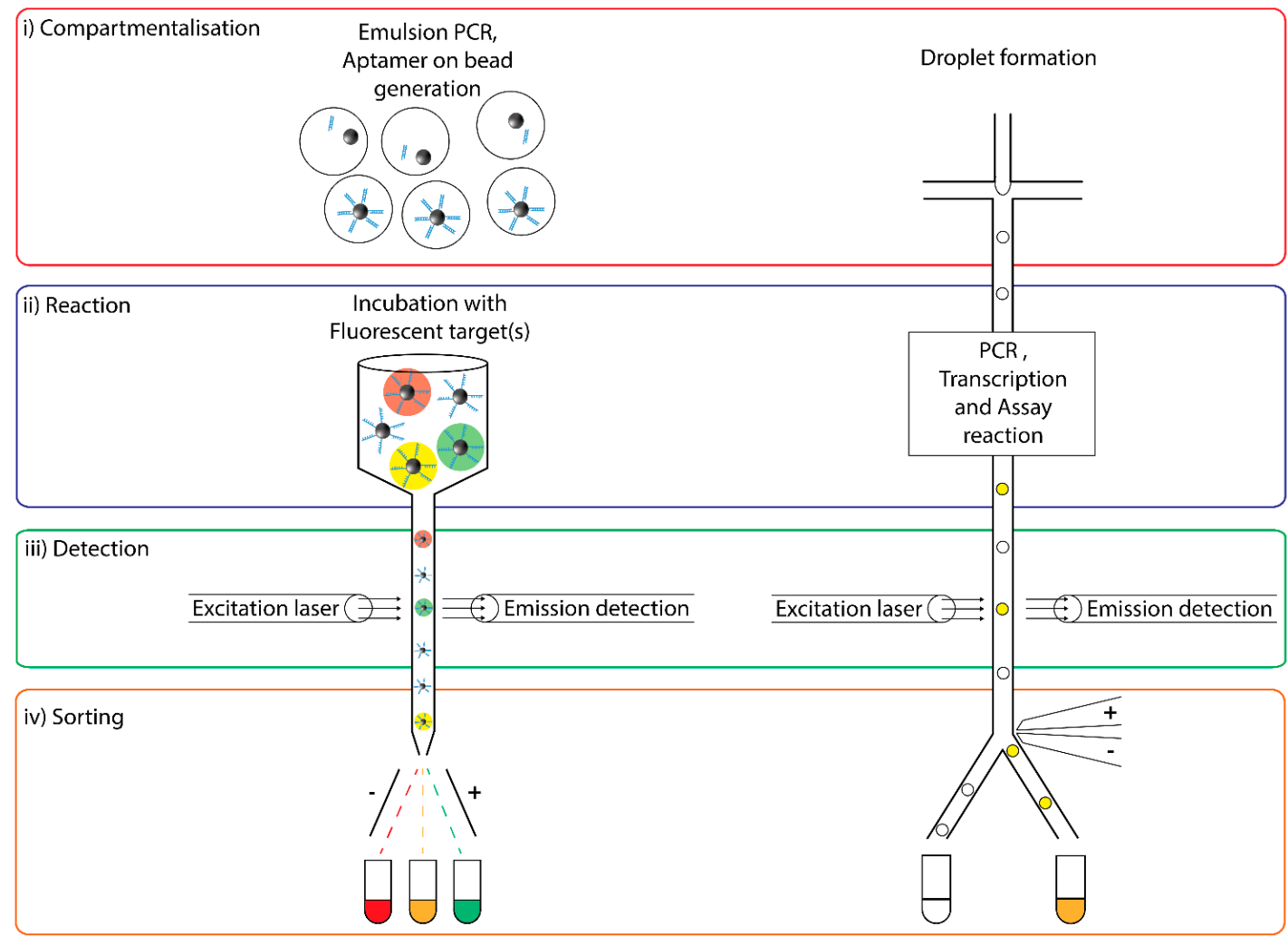

Figure 5: Comparison of (A) Flyorescence Activated Eell Sorting (EASES) and (B) Fluorescence

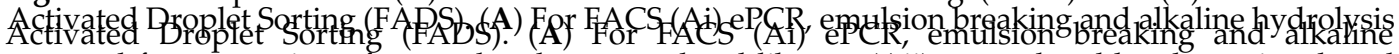

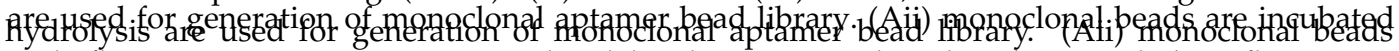

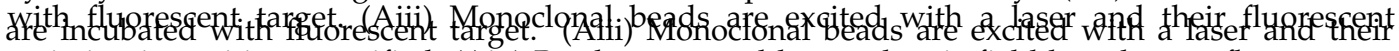

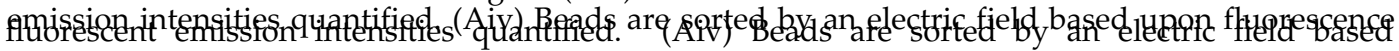

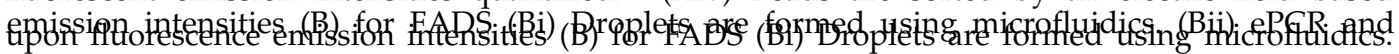

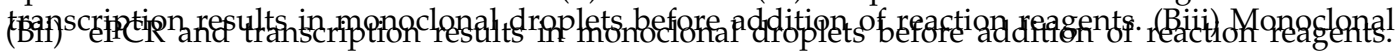

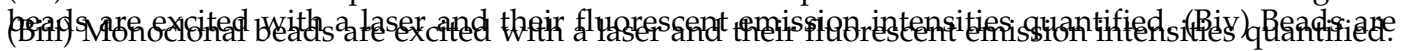

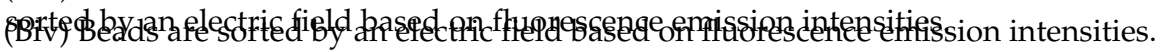

\subsection{1: Early Uses of FAES in Affinity Mglecule Selection}

The sorting p8wer of FAES for the isolation of affinity reagents was first used to select antibodies in directed evolution experiments [65. Yeast display is a technique where a libfary of antibodies is displayed gn veast cell purfaces by expression of an antibody and yeast surface fusion protein (Agazp): Follpwing incubation of the antibodiv-disphaving yeast cells with fluorescent target the cells expressing tight binding antibody could be selected using EAES [65]. Using this technigue, the selection of monoclonal antibodies was possible using FAES.

\section{3:1:2: FACS UsEd in Aptamer Selection}

With the advent of single species per bead oligonuleotide libraries [30] created by solid phase

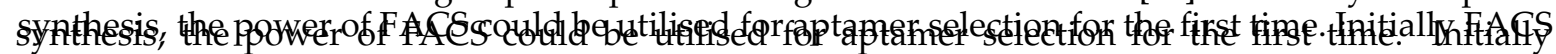
was just used as a post selection assay technique [32] but was later applied to the selection process [66]. As described previously, the inability to amplify these synthesised aptamer-on-bead libraries meant that only one round of selection could take pbacko4ingle round selection is a major drawback for FACS based selection techniques, although it may be possible using genetic algorithms to predict library sequences for subsequent rounds as seen in microarray aptamer selections [67].

The developments in ePCR, described in the section above, have allowed for the generation 
FACS was just used as a post selection assay technique [32] but was later applied to the selection process [66]. As described previously, the inability to amplify these synthesised aptamer-on-bead libraries meant that only one round of selection could take place. Single round selection is a major drawback for FACS based selection techniques, although it may be possible using genetic algorithms to predict library sequences for subsequent rounds as seen in microarray aptamer selections [67].

The developments in ePCR, described in the section above, have allowed for the generation monoclonal bead libraries [34]. This process, amenable to multiple rounds of selection, harnessed the true evolutionary power of SELEX and could now be used for FACS selection. Particle display is a term coined by Wang et al in 2014 [18]. The technique involves displaying aptamers on particles or beads similarly to the other monoclonal bead techniques [27]. The fundamental difference in the particle display paper was that FACS (Figure 3A) was used to sort the monoclonal beads.

Briefly, pre-enriched libraries were amplified using ePCR to create a monoclonal bead library. This library was then incubated with biotinylated target, washed and briefly incubated with streptavidin-phycoerythrin dye before undergoing selection using FACS (Figure $5 \mathrm{~A}$ ). The top $0.1 \%$ of monoclonal beads was selected and PCR was used to recover the selected oligonucleotide aptamers from the beads. This selection process was repeated three times.

To test this new approach aptamers were isolated against the four targets thrombin, ApoE, PAI-1, and 4-1BB. Aptamers against two of these targets, ApoE and thrombin, had already been reported so a comparison could be made between classical SELEX and monoclonal bead FACS selection. Aptamers isolated using this FACS selection had 1000 times stronger binding than aptamers isolated against the same targets using traditional SELEX [18]. For thrombin, aptamers with the extraordinarily tight binding dissociation constant of $7 \mathrm{pM}$ were isolated. For 4-1BB and PAI-1, previous attempts to isolate DNA aptamers against these targets had failed but here aptamers with tight binding dissociation constants of $2.32 \mathrm{nM}$ and $339 \mathrm{pM}$ were characterised respectively. Therefore FACS selection has a great potential to expand the range of aptamer targets currently accessible. These results show that FACS selection not only allows for the isolation of considerably tighter binding aptamers, but it can also increase the range of available targets suitable for aptamer selection.

\subsubsection{FACS Selection for Structure-Switching Aptamers}

Monoclonal microbeads can also be used to select for structure switching aptamer beacons, initially adapted to target steroids [16]. A monoclonal bead library of aptamers was made based on a previously selected aptamer containing a three-way junction centred on a random base-binding pocket. Two complementary sequences targeting adjacent sequence positions were synthesised: one with a conjugated fluorophore and one with a conjugated quencher. Once hybridised to the aptamer library, fluorescence is turned off due to the spatial proximity of the quencher. Upon incubation of the target with the fluorophore/quencher loaded monoclonal beads, any sequences in the library that bind the target with a significant structural change will displace the quencher sequence and cause a fluorescent signal to be exhibited. The monoclonal beads can then be sorted based on this fluorescent signal using FACS thereby selecting only for structure switching aptamer beacons.

The dissociation constant of the novel structure switching aptamers was slightly stronger $(4.1 \mu \mathrm{M})$ than that of the aptamer on which the initial library was based $(5 \mu \mathrm{M})$ [16]. These relatively high $K_{d}$ are understandable given the size and hydrophobicity of steroid molecules and the fact that simple unmodified DNA aptamers were used.

\subsubsection{Discussion of Similar Technologies}

FACS has been used to select aptamers using microparticles other than simple plastic microbeads such as agarose beads and even within living cells. For selection using agarose beads, monoclonal beads containing DNA aptamer libraries had to be first developed [17]. Briefly, agarose beads containing PCR reagents and precursor DNA aptamer templates were created using a microfluidic generated water-in-oil emulsion where the water phase contains PCR reagents, DNA aptamer 
template and agarose. After droplet formation, a thermal cycler was used to amplify the template within the droplets and then cooled to form solid agarose beads. Oligonucleotide-containing colonies were then easily separated using SYBR Green staining. The agarose bead library could then be incubated with a fluorescently labelled target and sorted using FACS to select for the tightest binding aptamers [17]. The disadvantage of using agarose beads over ePCR oligo-coated beads is that with agarose, the oligonucleotides are not covalently linked to the bead and so dissociation can occur over time. Since FACS selection of bead libraries can take up to several hours, there is the possibility that oligonucleotides could dissociate from the agarose beads, skewing the selection results.

Cells have also been used as a medium for oligonucleotide selection using FACS. Early research into fluorescent aptamers isolated RNA aptamers against the Spinach fluorophore using classical binding/capture selection [68]. The spinach fluorophore has no fluorescence in its native state however when a ligand binds and stabilises the structure, a strong fluorescent signal is produced. By inserting this RNA aptamer sequence into a gene of interest, this spinach/RNA aptamer system can be used in microscopy for live RNA tracking, similar to how GFP protein tracking is used in molecular biology. To improve upon these results, a selection system within bacterial cells was conducted to optimise the resulting RNA aptamers for functioning in cellular environments [69]. Briefly, DNA encoding an enriched spinach fluorophore binding RNA aptamer library was cloned into plasmids. The library of plasmids were transfected into a population of E. coli cells, incubated with the spinach fluorophore and any cells containing fluorophore activating RNA aptamers were selected using FACS. This selection process was repeated multiple times to isolate spinach fluorophore activating RNA aptamers. This FACS selection of aptamers in the cellular environment was very successful with the new Broccoli aptamers exhibiting 2-fold higher fluorescence when expressed in E. coli and compared to the original Spinach aptamers [69]. Despite the success of this technique in selecting aptamers within cells, selection is limited to RNA-based aptamers.

\subsection{Fluorescence Activated Droplet Sorting (FADS)}

As previously discussed, IVC confers multiple advantages by miniaturising chemical reactions into distinct picolitre volume reaction droplets. IVC droplets have been incorporated into FACS and since each droplet encapsulates a chemical reaction, enzymatic activity can be used as a sorting criterion. Selection of this enzymatic activity through IVC and FACS has given rise to methods of fluorescently activated droplet sorting FADS.

\subsubsection{IVC and FADS}

Within a single droplet, a range of in vitro reactions are possible such as transcription/translation, the expression of functional enzymes and bacterial synthesis of enzyme libraries. FADS can be used to sort the products of these reactions if fluorogenic substrates are incorporated into the reaction system. Unlike the selection techniques using FACS, which are designed to select simply for binding, enzymatic-based selection is more experimentally challenging. Fluorescent modifications, in both FACS and FADS, can lead to conformation changes of the target [70], and increase the likelihood of selecting spurious aptamers. However, many advances have been made in the field.

\subsubsection{From FACS to FADS: Optimising Droplet Sorting}

Fluorescence-activated droplet sorting (FADS) is a method of sorting emulsion droplets similar to FACS, first introduced in 2009 [71]. The microfluidics provide an efficient way to generate monodisperse emulsion droplets, and allows for greater manipulation of both the droplet contents as well as droplet size to the femtolitre scale (Figure 2) [72]. FADS experiments have been coupled with several droplet manipulation functions, such as: droplet splitting [73-75], droplet fusion [74,76-78], and picoinjection for the addition of reagents to existing droplets [79-81]. Similar to the principle of FACS, FADS utilises a microfluidic system to sort emulsion droplets based on their fluorescence intensities. Therefore droplets can be sorted according to the presence or absence of enzymatic activity 
with the incorporation of fluorogenic substrates. Similar to FACS, sorting can be implemented by creating an electric field across the sorting junctions where emulsion droplets of defined fluorescence intensity are deflected to the designated collection tube by dielectrophoresis (Figure 5, right). Selected emulsions can then be used for downstream analysis. Sorting can be achieved at an impressive $30 \mathrm{kHz}$ rate [82], although the more mature technology of FACS still holds superior sort rates [83]. For such enzymatic activity-based sorting applications, FADS eliminates the need to prepare cells and isolate them from aerosols produced in FACS sorting [84]. FADS has been used extensively in multiple applications such as the enhancement of the catalytic activity of enzymes, such as horseradish peroxidase [85], and the sorting of $\beta$-galactosidase genes in completely in vitro conditions [86]. This has given insight into the use of FADS to select catalytic oligonucleotides instead of simple binding aptamers.

\subsubsection{FADS to Select Oligonucleotides}

The selection of oligonucleotides within compartmentalised droplets for improved turnover rate has been achieved for ribozymes of varying functions $[87,88]$. Yet the polydispersity of droplets in these methods and the confines in throughput has limited selection efficacy. FADS has been used in the selection of ribozymes [25]. In previous ribozyme evolution experiments, the procedure relied on the self-modification of the RNA library, or ribozyme attachment to a substrate, followed by the enrichment of selected RNA molecules tethered to the product [89]. The selection process is based on intramolecular reactions in single turnover conditions in which ribozyme mutants with a high turnover rate are likely to be precluded. Mutations of a ribozyme's X-motif using error-prone PCR were used to generate a library of ribozyme mutants [25]. Individual variants were compartmentalised into monodisperse droplets along with fluorogenic substrates. In FADS, each step (e.g., library amplification, encapsulation of droplets and activity assay) is uncoupled which is suitable for directed evolution of most ribozymes working in intermolecular reactions or multiple turnover conditions [25]. The selected ribozyme variant exhibited a 28-times greater activity than the original ribozyme.

\subsubsection{Possible Use of FADS on Oligonucleotide Selection with Beads}

Previous work demonstrated the potential of in vitro selection and directed evolution of ribozymes by FADS technique [25]. Given the previously discussed advantages of monoclonal beads and $\mathrm{PPCR}$, the integration of catalytic nucleotide coated beads into a FADS system should allow for the selection of high-performance nucleotide enzymes. Since oligonucleotide-modified beads can be encapsulated inside microfluidic droplets [50], encapsulated oligonucleotide libraries can be easily incorporated into FADS systems [89]. Combining FADS with oligonucleotide-coated beads should be a powerful approach going forward for the aptamer field, particularly if combined with emerging XNA approaches [90].

\section{Conclusions}

Tethering oligonucleotides to beads through ePCR has increased the efficiency and versatility of established biotechnologies, including SELEX. The combination of monoclonal beads and FACS has led to robust aptamer selection techniques. One of the major disadvantages of classical binding/capture SELEX is the stochastic nature of the selection $[13,64]$. The use of FACS reduces this stochasticity, because every member in the library is individually assayed and sorted. This ensures that beneficial sequences are not lost and that detrimental sequences, such as nonspecific amplicons, are not preserved. Furthermore the efficiency of enrichment is substantially increased. Soh group's FACS selection of a monoclonal bead library method resulted in a $1.7 \times 10^{9}$ fold enrichment of an aptamer population within a single round. This is 100 million times better than the 10 -fold enrichment typically observed for a classical SELEX round. This in effect lowers the number of selection rounds required and the probability of candidate loss [18]. Although other media for FACS selection have 
been demonstrated [17,69], aptamer beads are practical for most aptamer selection applications. Their application to exotic selection techniques involving FACS, such as for structure switching aptamer beacons [16], present significant opportunities to expand existing approaches. FADS selection offers the tantalising opportunity to select not just for binding, but for higher complexity molecular events such as enzymatic activity.

Acknowledgments: The authors acknowledge funding for aptamer research under Hong Kong RGC GRF grants 778813M, 17119814 and 777109M.

Author Contributions: Lewis A. Fraser coordinated the writing, wrote Section 2.2, and collaborated in the creation of Figures 2-4; Andrew B. Kinghorn wrote Section 3.1 created Figure 5 and collaborated in the creation of Figure 1; Marco S.L. Tang: wrote Section 3.2; Yee-Wai Cheung: Collaborated in writing Section 1.; Bryce Lim wrote Section 2.2.1 and collaborated in the creation of Figures 2-4; Shaolin Liang: wrote Section 2.1, and collaborated in the creation of Figure 1; Roderick M. Dirkzwager: Collaborated in writing Section 1; Julian A. Tanner: Supervised the writing of all sections. All authors were involved in the editing process.

Conflicts of Interest: The authors declare no conflict of interest.

\section{References}

1. Ellington, A.D.; Szostak, J.W. In vitro selection of RNA molecules that bind specific ligands. Nature 1990, 346, 818-822. [CrossRef] [PubMed]

2. Tuerk, C.; Gold, L. Systematic evolution of ligands by exponential enrichment: RNA ligands to bacteriophage T4 DNA polymerase. Science 1990, 249, 505-510. [CrossRef] [PubMed]

3. Hofmann, H.; Limmer, S.; Hornung, V.; Sprinzl, M. $\mathrm{Ni}^{2+}$-binding RNA motifs with an asymmetric purine-rich internal loop and a G-A base pair. RNA 1997, 3, 1289-1300. [PubMed]

4. Rajendran, M.; Ellington, A.D. Selection of fluorescent aptamer beacons that light up in the presence of zinc. Anal. Bioanal. Chem. 2008, 390, 1067-1075. [CrossRef] [PubMed]

5. Liu, M.; Kagahara, T.; Abe, H.; Ito, Y. Direct in vitro selection of hemin-binding DNA aptamer with peroxidase activity. Bull. Chem. Soc. Jpn. 2009, 82, 99-104. [CrossRef]

6. Stojanovic, M.N.; de Prada, P.; Landry, D.W. Fluorescent sensors based on aptamer self-assembly. J. Am. Chem. Soc. 2000, 122, 11547-11548. [CrossRef]

7. Cheung, Y.W.; Kwok, J.; Law, A.W.L.; Watt, R.M.; Kotaka, M.; Tanner, J.A. Structural basis for discriminatory recognition of plasmodium lactate dehydrogenase by a DNA aptamer. Proc. Natl. Acad. Sci. USA 2013, 110, 15967-15972. [CrossRef] [PubMed]

8. Long, S.B.; Long, M.B.; White, R.R.; Sullenger, B.A. Crystal structure of an RNA aptamer bound to thrombin. RNA 2008, 14, 2504-2512. [CrossRef] [PubMed]

9. Morris, K.N.; Jensen, K.B.; Julin, C.M.; Weil, M.; Gold, L. High affinity ligands from in vitro selection: Complex targets. Proc. Natl. Acad. Sci. USA 1998, 95, 2902-2907. [CrossRef] [PubMed]

10. Shangguan, D.; Li, Y.; Tang, Z.; Cao, Z.C.; Chen, H.W.; Mallikaratchy, P.; Sefah, K.; Yang, C.J.; Tan, W. Aptamers evolved from live cells as effective molecular probes for cancer study. Proc. Natl. Acad. Sci. USA 2006, 103, 11838-11843. [CrossRef] [PubMed]

11. Jayasena, S.D. Aptamers: An emerging class of molecules that rival antibodies in diagnostics. Clin. Chem. 1999, 45, 1628-1650. [PubMed]

12. Irvine, D.; Tuerk, C.; Gold, L. SELEXION. Systematic evolution of ligands by exponential enrichment with integrated optimization by non-linear analysis. J. Mol. Biol. 1991, 222, 739-761. [CrossRef]

13. Vant-Hull, B.; Payano-Baez, A.; Davis, R.H.; Gold, L. The mathematics of SELEX against complex targets. J. Mol. Biol. 1998, 278, 579-597. [CrossRef] [PubMed]

14. Chen, C.-K. Complex SELEX against target mixture: Stochastic computer model, simulation, and analysis. Comput. Methods Programs Biomed. 2007, 87, 189-200. [CrossRef] [PubMed]

15. Hoinka, J.; Berezhnoy, A.; Dao, P.; Sauna, Z.E.; Gilboa, E.; Przytycka, T.M. Large scale analysis of the mutational landscape in HT-SELEX improves aptamer discovery. Nucleic Acids Res. 2015, 43, 5699-5707. [CrossRef] [PubMed]

16. Trevino, S.G.; Levy, M. High-throughput bead-based identification of structure-switching aptamer beacons. ChemBioChem 2014, 15, 1877-1881. [CrossRef] [PubMed] 
17. Zhang, W.Y.; Zhang, W.; Liu, Z.; Li, C.; Zhu, Z.; Yang, C.J. Highly parallel single-molecule amplification approach based on agarose droplet polymerase chain reaction for efficient and cost-effective aptamer selection. Anal. Chem. 2011, 84, 350-355. [CrossRef] [PubMed]

18. Wang, J.; Gong, Q.; Maheshwari, N.; Eisenstein, M.; Arcila, M.L.; Kosik, K.S.; Soh, H.T. Particle display: A quantitative screening method for generating high-affinity aptamers. Angew. Chem. Int. Ed. 2014, 53, 4796-4801. [CrossRef] [PubMed]

19. Rödiger, S.; Liebsch, C.; Schmidt, C.; Lehmann, W.; Resch-Genger, U.; Schedler, U.; Schierack, P. Nucleic acid detection based on the use of microbeads: A review. Microchim. Acta 2014, 181, 1151-1168. [CrossRef]

20. Shum, K.T.; Lui, E.L.H.; Wong, S.C.K.; Yeung, P.; Sam, L.; Wang, Y.; Watt, R.M.; Tanner, J.A. Aptamer-mediated inhibition of mycobacterium tuberculosis polyphosphate kinase 2. Biochemistry 2011, 50, 3261-3271. [CrossRef] [PubMed]

21. Shum, K.T.; Tanner, J.A. Differential inhibitory activities and stabilisation of DNA aptamers against the sars coronavirus helicase. ChemBioChem 2008, 9, 3037-3045. [CrossRef] [PubMed]

22. Hamm, J. Characterisation of antibody-binding rnas selected from structurally constrained libraries. Nucleic Acids Res. 1996, 24, 2220-2227. [CrossRef] [PubMed]

23. Walter, J.-G.; Kökpinar, O.; Friehs, K.; Stahl, F.; Scheper, T. Systematic investigation of optimal aptamer immobilization for protein-Microarray applications. Anal. Chem. 2008, 80, 7372-7378. [CrossRef] [PubMed]

24. Herzenberg, L.A.; Parks, D.; Sahaf, B.; Perez, O.; Roederer, M.; Herzenberg, L.A. The history and future of the fluorescence activated cell sorter and flow cytometry: A view from stanford. Clin. Chem. 2002, 48, 1819-1827. [PubMed]

25. Ryckelynck, M.; Baudrey, S.; Rick, C.; Marin, A.; Coldren, F.; Westhof, E.; Griffiths, A.D. Using droplet-based microfluidics to improve the catalytic properties of RNA under multiple-turnover conditions. RNA 2015, 21, 458-469. [CrossRef] [PubMed]

26. Hünniger, T.; Wessels, H.; Fischer, C.; Paschke-Kratzin, A.; Fischer, M. Just in time-selection: A rapid semiautomated SELEX of DNA aptamers using magnetic separation and BEAMing. Anal. Chem. 2014, 86, 10940-10947. [CrossRef] [PubMed]

27. Zhu, Z.; Song, Y.; Li, C.; Zou, Y.; Zhu, L.; An, Y.; Yang, C.J. Monoclonal surface display SELEX for simple, rapid, efficient, and cost-effective aptamer enrichment and identification. Anal. Chem. 2014, 86, 5881-5888. [CrossRef] [PubMed]

28. Furka, A.; Sebestyén, F.; Asgedom, M.; Dibó, G. General method for rapid synthesis of multicomponent peptide mixtures. Int. J. Pept. Protein Res. 1991, 37, 487-493. [CrossRef] [PubMed]

29. Lam, K.S.; Salmon, S.E.; Hersh, E.M.; Hruby, V.J.; Kazmierski, W.M.; Knapp, R.J. A new type of synthetic peptide library for identifying ligand-binding activity. Nature 1991, 354, 82-84. [CrossRef] [PubMed]

30. Yang, X.; Bassett, S.E.; Li, X.; Luxon, B.A.; Herzog, N.K.; Shope, R.E.; Aronson, J.; Prow, T.W.; Leary, J.F.; Kirby, R. Construction and selection of bead-bound combinatorial oligonucleoside phosphorothioate and phosphorodithioate aptamer libraries designed for rapid PCR-based sequencing. Nucleic Acids Res. 2002, 30, e132. [CrossRef] [PubMed]

31. Lam, K.S.; Lebl, M.; Krchnák, V. The "one-bead-one-compound" combinatorial library method. Chem. Rev. 1997, 97, 411-448. [CrossRef] [PubMed]

32. Yang, X.; Li, X.; Prow, T.W.; Reece, L.M.; Bassett, S.E.; Luxon, B.A.; Herzog, N.K.; Aronson, J.; Shope, R.E.; Leary, J.F. Immunofluorescence assay and flow-cytometry selection of bead-bound aptamers. Nucleic Acids Res. 2003, 31, e54. [CrossRef] [PubMed]

33. Tawfik, D.S.; Griffiths, A.D. Man-made cell-like compartments for molecular evolution. Nat. Biotechnol. 1998, 16, 652-656. [CrossRef] [PubMed]

34. Nakano, M.; Komatsu, J.; Matsuura, S.-I.; Takashima, K.; Katsura, S.; Mizuno, A. Single-molecule PCR using water-in-oil emulsion. J. Biotechnol. 2003, 102, 117-124. [CrossRef]

35. Shao, K.; Ding, W.; Wang, F.; Li, H.; Ma, D.; Wang, H. Emulsion PCR: A high efficient way of PCR amplification of random DNA libraries in aptamer selection. PLoS ONE 2011, 6, e24910. [CrossRef] [PubMed]

36. Schütze, T.; Rubelt, F.; Repkow, J.; Greiner, N.; Erdmann, V.A.; Lehrach, H.; Konthur, Z.; Glökler, J. A streamlined protocol for emulsion polymerase chain reaction and subsequent purification. Anal. Biochem. 2011, 410, 155-157. [CrossRef] [PubMed] 
37. Williams, R.; Peisajovich, S.G.; Miller, O.J.; Magdassi, S.; Tawfik, D.S.; Griffiths, A.D. Amplification of complex gene libraries by emulsion PCR. Nat. Methods 2006, 3, 545-550. [CrossRef] [PubMed]

38. Meyerhans, A.; Vartanian, J.-P.; Wain-Hobson, S. DNA recombination during PCR. Nucleic Acids Res. 1990, 18, 1687-1691. [CrossRef] [PubMed]

39. Nakano, H.; Kobayashi, K.; Ohuchi, S.; Sekiguchi, S.; Yamane, T. Single-step single-molecule PCR of DNA with a homo-priming sequence using a single primer and hot-startable DNA polymerase. J. Biosci. Bioeng. 2000, 90, 456-458. [CrossRef]

40. Taly, V.; Kelly, B.T.; Griffiths, A.D. Droplets as microreactors for high-throughput biology. ChemBioChem 2007, 8, 263-272. [CrossRef] [PubMed]

41. Song, H.; Chen, D.L.; Ismagilov, R.F. Reactions in droplets in microfluidic channels. Angew. Chem. Int. Ed. 2006, 45, 7336-7356. [CrossRef] [PubMed]

42. Duffy, D.C.; McDonald, J.C.; Schueller, O.J.; Whitesides, G.M. Rapid prototyping of microfluidic systems in poly (dimethylsiloxane). Anal. Chem. 1998, 70, 4974-4984. [CrossRef] [PubMed]

43. Diehl, F.; Li, M.; He, Y.; Kinzler, K.W.; Vogelstein, B.; Dressman, D. BEAMing: Single-molecule PCR on microparticles in water-in-oil emulsions. Nat. Methods 2006, 3, 551-559. [CrossRef] [PubMed]

44. Leng, X.; Zhang, W.; Wang, C.; Cui, L.; Yang, C.J. Agarose droplet microfluidics for highly parallel and efficient single molecule emulsion PCR. Lab Chip 2010, 10, 2841-2843. [CrossRef] [PubMed]

45. Baier, G.; Musyanovych, A.; Landfester, K.; Best, A.; Lorenz, S.; Mailänder, V. DNA amplification via polymerase chain reaction inside miniemulsion droplets with subsequent poly ( $n$-butylcyanoacrylate) shell formation and delivery of polymeric capsules into mammalian cells. Macromol. Biosci. 2011, 11, 1099-1109. [CrossRef] [PubMed]

46. Yufa, R.; Krylova, S.; Bruce, C.; Bagg, E.; Schofield, C.; Krylov, S. Emulsion PCR significantly improves nonequilibrium capillary electrophoresis of equilibrium mixtures-based aptamer selection: Allowing for efficient and rapid selection of aptamer to unmodified ABH2 protein. Anal. Chem. 2014, 87, 1411-1419. [CrossRef] [PubMed]

47. Diehl, F.; Schmidt, K.; Choti, M.A.; Romans, K.; Goodman, S.; Li, M.; Thornton, K.; Agrawal, N.; Sokoll, L.; Szabo, S.A. Circulating mutant DNA to assess tumor dynamics. Nat. Med. 2008, 14, 985-990. [CrossRef] [PubMed]

48. Richardson, A.L.; Iglehart, J.D. BEAMing up personalized medicine: Mutation detection in blood. Clin. Cancer Res. 2012, 18, 3209-3211. [CrossRef] [PubMed]

49. Chen, W.W.; Balaj, L.; Liau, L.M.; Samuels, M.L.; Kotsopoulos, S.K.; Maguire, C.A.; LoGuidice, L.; Soto, H.; Garrett, M.; Zhu, L.D. BEAMing and droplet digital PCR analysis of mutant IDH1 mRNA in glioma patient serum and cerebrospinal fluid extracellular vesicles. Mol. Ther. Nucleic Acids 2013, 2, e109. [CrossRef] [PubMed]

50. Dressman, D.; Yan, H.; Traverso, G.; Kinzler, K.W.; Vogelstein, B. Transforming single DNA molecules into fluorescent magnetic particles for detection and enumeration of genetic variations. Proc. Natl. Acad. Sci. USA 2003, 100, 8817-8822. [CrossRef] [PubMed]

51. Li, M.; Diehl, F.; Dressman, D.; Vogelstein, B.; Kinzler, K.W. BEAMing up for detection and quantification of rare sequence variants. Nat. Methods 2006, 3, 95-97. [CrossRef] [PubMed]

52. Okochi, M.; Tsuchiya, H.; Kumazawa, F.; Shikida, M.; Honda, H. Droplet-based gene expression analysis using a device with magnetic force-based-droplet-handling system. J. Biosci. Bioeng. 2010, 109, 193-197. [CrossRef] [PubMed]

53. Zeng, Y.; Novak, R.; Shuga, J.; Smith, M.T.; Mathies, R.A. High-performance single cell genetic analysis using microfluidic emulsion generator arrays. Anal. Chem. 2010, 82, 3183-3190. [CrossRef] [PubMed]

54. Wang, Y.; Lu, J.; Yang, Q.; Bai, Y.; Ge, Q. An emulsion based microarray method to detect the toxin genes of toxin-producing organisms. Molecules 2011, 16, 7365-7376. [CrossRef] [PubMed]

55. Levy, M.; Griswold, K.E.; Ellington, A.D. Direct selection of trans-acting ligase ribozymes by in vitro compartmentalization. RNA 2005, 11, 1555-1562. [CrossRef] [PubMed]

56. Shendure, J.; Porreca, G.J.; Reppas, N.B.; Lin, X.; McCutcheon, J.P.; Rosenbaum, A.M.; Wang, M.D.; Zhang, K.; Mitra, R.D.; Church, G.M. Accurate multiplex polony sequencing of an evolved bacterial genome. Science 2005, 309, 1728-1732. [CrossRef] [PubMed] 
57. Margulies, M.; Egholm, M.; Altman, W.E.; Attiya, S.; Bader, J.S.; Bemben, L.A.; Berka, J.; Braverman, M.S.; Chen, Y.-J.; Chen, Z. Genome sequencing in microfabricated high-density picolitre reactors. Nature 2005, 437, 376-380. [CrossRef] [PubMed]

58. Valouev, A.; Ichikawa, J.; Tonthat, T.; Stuart, J.; Ranade, S.; Peckham, H.; Zeng, K.; Malek, J.A.; Costa, G.; McKernan, K. A high-resolution, nucleosome position map of C. elegans reveals a lack of universal sequence-dictated positioning. Genome Res. 2008, 18, 1051-1063. [CrossRef] [PubMed]

59. Xu, M.Y.; Aragon, A.D.; Mascarenas, M.R.; Torrez-Martinez, N.; Edwards, J.S. Dual primer emulsion PCR for next-generation DNA sequencing. Biotechniques 2010, 48, 409-412. [CrossRef] [PubMed]

60. Rotman, B. Measurement of activity of single molecules of $\beta$-D-galactosidase. Proc. Natl. Acad. Sci. USA 1961, 47, 1981-1991. [CrossRef] [PubMed]

61. Sepp, A.; Tawfik, D.S.; Griffiths, A.D. Microbead display by in vitro compartmentalisation: Selection for binding using flow cytometry. FEBS Lett. 2002, 532, 455-458. [CrossRef]

62. Griffiths, A.D.; Tawfik, D.S. Directed evolution of an extremely fast phosphotriesterase by in vitro compartmentalization. EMBO J. 2003, 22, 24-35. [CrossRef] [PubMed]

63. Herzenberg, L.A.; Sweet, R.G.; Herzenberg, L.A. Fluorescence-activated cell sorting. Sci. Am. 1976, 234, 108-117. [CrossRef] [PubMed]

64. Mencin, N.; Šmuc, T.; Vraničar, M.; Mavri, J.; Hren, M.; Galeša, K.; Krkoč, P.; Ulrich, H.; Šolar, B. Optimization of SELEX: Comparison of different methods for monitoring the progress of in vitro selection of aptamers. J. Pharm. Biomed. Anal. 2014, 91, 151-159. [CrossRef] [PubMed]

65. Boder, E.T.; Wittrup, K.D. Yeast surface display for screening combinatorial polypeptide libraries. Nat. Biotechnol. 1997, 15, 553-557. [CrossRef] [PubMed]

66. Leary, J.F. Ultra high-speed sorting. Cytometry A 2005, 67, 76-85. [CrossRef] [PubMed]

67. Platt, M.; Rowe, W.; Wedge, D.C.; Kell, D.B.; Knowles, J.; Day, P.J. Aptamer evolution for array-based diagnostics. Anal. Biochem. 2009, 390, 203-205. [CrossRef] [PubMed]

68. Paige, J.S.; Wu, K.Y.; Jaffrey, S.R. RNA mimics of green fluorescent protein. Science 2011, 333, $642-646$. [CrossRef] [PubMed]

69. Filonov, G.S.; Moon, J.D.; Svensen, N.; Jaffrey, S.R. Broccoli: Rapid selection of an RNA mimic of green fluorescent protein by fluorescence-based selection and directed evolution. J. Am. Chem. Soc. 2014, 136, 16299-16308. [CrossRef] [PubMed]

70. Toseland, C.P. Fluorescent labeling and modification of proteins. J. Chem Biol. 2013, 6, 85-95. [CrossRef] [PubMed]

71. Baret, J.-C.; Miller, O.J.; Taly, V.; Ryckelynck, M.; El-Harrak, A.; Frenz, L.; Rick, C.; Samuels, M.L.; Hutchison, J.B.; Agresti, J.J. Fluorescence-activated droplet sorting (FADS): Efficient microfluidic cell sorting based on enzymatic activity. Lab Chip 2009, 9, 1850-1858. [CrossRef] [PubMed]

72. Leman, M.; Abouakil, F.; Griffiths, A.D.; Tabeling, P. Droplet-based microfluidics at the femtolitre scale. Lab Chip 2015, 15, 753-765. [CrossRef] [PubMed]

73. Link, D.; Anna, S.L.; Weitz, D.; Stone, H. Geometrically mediated breakup of drops in microfluidic devices. Phys. Rev. Lett. 2004, 92, 054503. [CrossRef] [PubMed]

74. Knight, C.G.; Platt, M.; Rowe, W.; Wedge, D.C.; Khan, F.; Day, P.J.; McShea, A.; Knowles, J.; Kell, D.B. Array-based evolution of DNA aptamers allows modelling of an explicit sequence-fitness landscape. Nucleic Acids Res. 2009, 37, e6. [CrossRef] [PubMed]

75. Abate, A.R.; Weitz, D.A. Faster multiple emulsification with drop splitting. Lab Chip 2011, 11, $1911-1915$. [CrossRef] [PubMed]

76. Ahn, K.; Agresti, J.; Chong, H.; Marquez, M.; Weitz, D. Electrocoalescence of drops synchronized by size-dependent flow in microfluidic channels. Appl. Phys. Lett. 2006, 88, 264105. [CrossRef]

77. Zagnoni, M.; le Lain, G.; Cooper, J.M. Electrocoalescence mechanisms of microdroplets using localized electric fields in microfluidic channels. Langmuir 2010, 26, 14443-14449. [CrossRef] [PubMed]

78. Guzman, A.R.; Kim, H.S.; de Figueiredo, P.; Han, A. A three-dimensional electrode for highly efficient electrocoalescence-based droplet merging. Biomed. Microdevices 2015, 17, 1-9. [CrossRef] [PubMed]

79. Abate, A.R.; Hung, T.; Mary, P.; Agresti, J.J.; Weitz, D.A. High-throughput injection with microfluidics using picoinjectors. Proc. Natl. Acad. Sci. USA 2010, 107, 19163-19166. [CrossRef] [PubMed]

80. O’Donovan, B.; Eastburn, D.J.; Abate, A.R. Electrode-free picoinjection of microfluidic drops. Lab Chip 2012, 12, 4029-4032. [CrossRef] [PubMed] 
81. Rhee, M.; Light, Y.K.; Yilmaz, S.; Adams, P.D.; Saxena, D.; Meagher, R.J.; Singh, A.K. Pressure stabilizer for reproducible picoinjection in droplet microfluidic systems. Lab Chip 2014, 14, 4533-4539. [CrossRef] [PubMed]

82. Sciambi, A.; Abate, A.R. Accurate microfluidic sorting of droplets at $30 \mathrm{khz}$. Lab Chip 2015, 15, 47-51. [CrossRef] [PubMed]

83. Arnold, L.W.; Lannigan, J. Practical issues in high-speed cell sorting. Curr. Protoc. Cytom. 2010, 51, 1-24.

84. Holmes, K.L. Characterization of aerosols produced by cell sorters and evaluation of containment. Cytometry A 2011, 79, 1000-1008. [CrossRef] [PubMed]

85. Agresti, J.J.; Antipov, E.; Abate, A.R.; Ahn, K.; Rowat, A.C.; Baret, J.-C.; Marquez, M.; Klibanov, A.M.; Griffiths, A.D.; Weitz, D.A. Ultrahigh-throughput screening in drop-based microfluidics for directed evolution. Proc. Natl. Acad. Sci. USA 2010, 107, 4004-4009. [CrossRef] [PubMed]

86. Fallah-Araghi, A.; Baret, J.-C.; Ryckelynck, M.; Griffiths, A.D. A completely in vitro ultrahigh-throughput droplet-based microfluidic screening system for protein engineering and directed evolution. Lab Chip 2012, 12, 882-891. [CrossRef] [PubMed]

87. Agresti, J.J.; Kelly, B.T.; Jäschke, A.; Griffiths, A.D. Selection of ribozymes that catalyse multiple-turnover diels-alder cycloadditions by using in vitro compartmentalization. Proc. Natl. Acad. Sci. USA 2005, 102, 16170-16175. [CrossRef] [PubMed]

88. Wochner, A.; Attwater, J.; Coulson, A.; Holliger, P. Ribozyme-catalyzed transcription of an active ribozyme. Science 2011, 332, 209-212. [CrossRef] [PubMed]

89. Bartel, D.P.; Szostak, J.W. Isolation of new ribozymes from a large pool of random sequences. Science 1993, 261, 1411-1418. [CrossRef] [PubMed]

90. Taylor, A.I.; Pinheiro, V.B.; Smola, M.J.; Morgunov, A.S.; Peak-Chew, S.; Cozens, C.; Weeks, K.M.; Herdewijn, P.; Holliger, P. Catalysts from synthetic genetic polymers. Nature 2015, 518, 427-430. [CrossRef] [PubMed]

(C) 2015 by the authors; licensee MDPI, Basel, Switzerland. This article is an open access article distributed under the terms and conditions of the Creative Commons by Attribution (CC-BY) license (http://creativecommons.org/licenses/by/4.0/). 